\title{
AリII02 I45961
}

NATL INST OF STANDARDS \& TECH R.I.C.

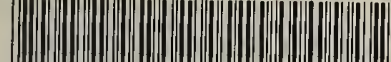

INSRDS-NBS 11102145961

QC100 U573 V25;1968 C.2 NBS-PUB-C 1964

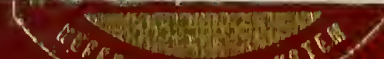

peponet with

I.

\section{NBS}

\section{PUBLICATIONS}

Electron Impact

Excitation of Atoms

$Q G$

100

4573

no.25

1968

c. 3
D.S. DEPARTWENT OF COMMEREE

MATANAL PUREMU OF STAMDAROS

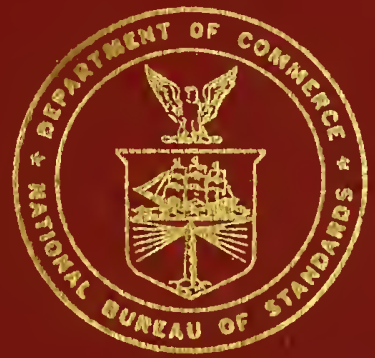





MAY9 1974 UNITED STATES DEPARTMENT OF COMMERCE
C. R. SMITH, Secretary

$110 ? 21$

NATIONAL BUREAU OF STANDARDS, A. V. Astin, Director

\title{
Electron Impact Excitation of Atoms
}

\author{
B. L. Moiseiwitsch \\ Department of Applied Mathematics \\ The Queen's University of Belfast \\ Belfast, Northern Ireland
}

AN D

\section{S. J. Smith}

Laboratory Astrophysics Division

National Bureau of Standards

Boulder, Colorado 80302
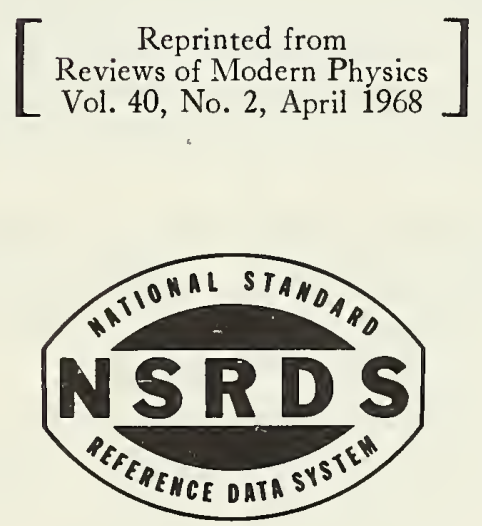

NSRDS-NBS 25

National Standard Reference Data Series-

National Bureau of Standards 25

Issued August 1968

For sale by the Superintendent of Documents, U.S. Government Printing Office Washington, D.C. 20402 - Price $\$ 2$ 


\section{Foreword}

The National Standard Reference Data System is a Government-wide effort to provide for the technical community of the United States effective access to the quantitative data of physical science, critically evaluated and compiled for convenience, and readily accessible through a variety of distribution channels. The System was established in 1963 by action of the President's Office of Science and Technology and the Federal Council for Science and Technology.

The responsibility to administer the System was assigned to the National Bureau of Standards and an Office of Standard Reference Data was set up at the Bureau for this purpose. Since 1963, this Office has developed systematic plans for meeting high-priority needs for reliable reference data. It has undertaken to coordinate and integrate existing data evaluation and compilation activities (primarily those under sponsorship of Federal agencies) into a comprehensive program, supplementing and expanding technical coverage when necessary, establishing and maintaining standards for the output of the participating groups, and providing mechanisms for the dissemination of the output as required.

The System now comprises a complex of data centers and other activities, carried on in Government agencies, academic institutions, and nongovernmental laboratories. The independent operational status of existing critical data projects is maintained and encouraged. Data centers that are components of the NSRDS produce compilations of critically evaluated data, critical reviews of the state of quantitative knowledge in specialized areas, and computations of useful functions derived from standard reference data. In addition, the centers and projects establish criteria for evaluation and compilation of data and make recommendations on needed modifications or extensions of experimental techniques.

Data publications of the NSRDS take a variety of physical forms, including books, pamphlets, loose-leaf sheets and computer tapes. While most of the compilations have been issued by the Government Printing Office, several have appeared in scientific journals. Under some circumstances, private publishing houses are regarded as appropriate primary dissemination mechanisms.

The technical scope of the NSRDS is indicated by the principal categories of data compilation projects now active or being planned: nuclear properties, atomic and molecular properties, solid state properties, thermodynamic and transport properties, chemical kinetics, colloid and surface properties, and mechanical properties.

An important aspect of the NSRDS is the advice and planning assistance which the National Research Council of the National Academy of Sciences-National Academy of Engineering provides. These services are organized under an overall Review Committee which considers the program as a whole and makes recommendations on policy, long-term planning, and international collaboration. Advisory Panels, each concerned with a single technical area, meet regularly to examine major portions of the program, assign relative priorities, and identify specific key problems in need of further attention. For selected specific topics, the Advisory Panels sponsor subpanels which make detailed studies of users' needs, the present state of knowledge, and existing data resources as a basis for recommending one or more data compilation activities. This assembly of advisory services contributes greatly to the guidance of NSRDS activities.

The NSRDS-NBS series of publications is intended primarily to include evaluated reference data and critical reviews of long-term interest to the scientific and technical community. 


\section{Preface}

The publication philosophy of the National Standard Reference Data System recognizes that data compilations will be most useful if all available channels of publishing and disseminating the information are employed. Selection of a specific channel - Government Printing Office, a scientific journal, or a commercial publishing house - is determined by the circumstances for the individual document concerned. The goal is to reach all of the appropriate audience most readily at minimum expense.

The critical review which follows was first published in Reviews of Modern Physics and is part of the JILA Information Center's program of compilation and critical evaluation of low energy atomic collision data. The authors and editors felt that this journal would reach the intended readers, and the Office of Standard Reference Data agreed that this channel of publication promoted the objectives of the National Standard Reference Data System. In addition, however, all concerned recognized that wider distribution and the capability of providing a continuing source of supply could be afforded by prompt republication through the Government Printing Office.

With the generous permission of the editors of Reviews of Modern Physics, and the approval of the authors, the Office of Standard Reference Data has undertaken to reprint this article as a part of the National Standard Reference Data System - National Bureau of Standards series.

Anyone wishing information about the availability of other publications of this type from the JILA Information Center should contact:

Dr. L. J. Kieffer, Director

JILA Information Center

Joint Institute for Laboratory

Astrophysics

University of Colorado

Boulder, Colo. 80302. 


\begin{abstract}
The experimental and theoretical literature about the electron impact excitation of atoms is reviewed. Theoretical methods ranging from the Bethe and Born approximations to the close coupling approximations are discussed and intercompared. Where possible, on theoretical grounds or through intercomparison, the reliability of the various methods is discussed.

A general critique of the optical method of measuring excitation functions is given, with the objective of promoting higher quality future experimental work. A critical study of existing experimental work leads to the conclusion that most workers have ignored important physical and instrumental effects, and it may be presumed that the data in the literature is subject to many unrecognized systematic errors. The literature on hydrogen and helium is discussed critically. The literature on the alkalis, heavy rare gases, mercury; cadmium and zinc is surveyed but the quality of the literature does not support critical review beyond some general comments about the physics of these atoms.
\end{abstract}

Key words: Atom, electron, excitation, experimental, helium, hydrogen, impact, review, theoretical.

\title{
Contents
}

Foreword

Page

Preface.

III

Contents to paper

Introduction 


\title{
Electron Impact Excitation of Atoms
}

\author{
B. L. MOISEIWITSCH \\ Department of Applied Mathemalics, The Queen's University of Belfast, Belfast, Northern Ireland
}

S. J. SMITH*

Joint Institule for Laboratory Astrophysics, $\dagger$ Boulder, Colorado

\section{CONTENTS}

I. Theory $\ldots \ldots \ldots \ldots \ldots \ldots \ldots \ldots \ldots \ldots \ldots \ldots \ldots \ldots, 1$

1. Introduction. ....................... 1

2. Hydrogen Atoms. . . . . . . . . . . . . . 2

2.1. First Born Approximation............ 2

2.2. Bethe Approximation............. 7

2.3. Second Born Approximation.......... 8

2.4. Exchange Scattering............... 9

2.5. Born-Oppenheimer Approximation........ 10

2.6. First-Order Exchange Approximation ....... 10

2.7. Ochkur Approximation............... 11

2.8. Distorted Waves Approximation........ 12

2.9. Modified Bethe Approximation.......... 14

2.10. Impact Parameter Method ............. 14

2.11. Classical Approximation............. 15

2.12. Vainshtein, Presnyakov, Sobelman Approxi-

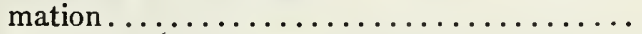

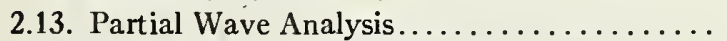

2.14. Unitarized Born Approximation. . . . . . . . .

2.15. Close Coupling Approximations. . . . . . . . .

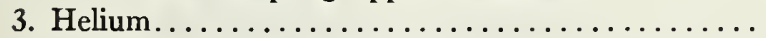

3.1. First Born Approximation . . . . . . . . . 21

3.2. Exchange Approximations.............. 23

3.3. Distorted Waves Approximation......... 26

3.4. Close Coupling Approximation.......... 26

3.5. Double Excitation of Helium........... 27

4. Neon and Argon................... 28

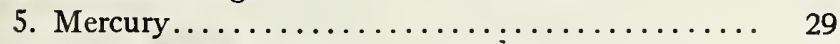

6. Alkali Metal Atoms.................. 29

7. Oxygen and Nitrogen Atoms............. 31

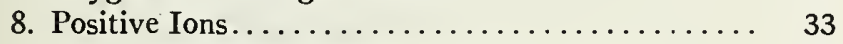

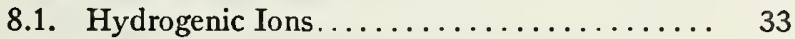

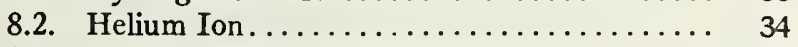

8.3. Helium-Like Ions............... 35

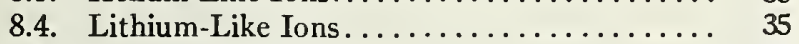

8.5. Sodium-Like Ions................... 36

8.6. Potassium-Like Ions................. 36

8.7. Positive Ions Having $p^{2}, p^{3}$, or $p^{4}$ Configura-

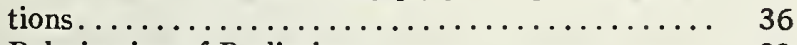

9. Polarization of Radiation ................ 38

10. Theoretical Summary.................. 39

II. Experiment.......................... 40

11. Introduction . . . . . . . . . . 40

12. Experimental Methods................. 41

12.1. Pressure Dependence of Excitation Functions 42

12.2. The Effects of Polarization........... 46

12.3. Electric Field Effects................. 49

12.4. Absolute Measurements of Optical Excitation

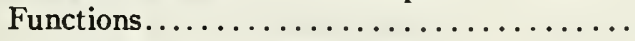

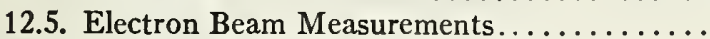

13. Other Reviews of Electron Impact Excitation....

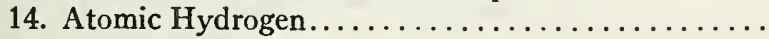

14.1. Excitation of $H(2 p) \ldots \ldots \ldots \ldots \ldots \ldots \ldots$

14.2. Excitation of $H(2 s) \ldots \ldots \ldots \ldots \ldots \ldots \ldots$

14.3. Excitation of Balmer Lines of Hydrogen....

\section{0}

51

51

51

52

54

56

* Staff member, Laboratory Astrophysics Division, National Bureau of Standards.

$\dagger$ Of the National Bureau of Standards and the University of Colorado.
15. Excitation of Helium Positive Ions.......... 56

16. Helium......................... 56

16.1. Helium $n^{1} P \rightarrow 2^{1} S$ Transitions .......... 56

16.2. Helium $n^{1} S \rightarrow 2^{1} P$ Transitions.......... 63

16.3. Helium $n^{1} D \rightarrow 2^{1} P$ Transitions . . . . . . . . 65

16.4. Helium $n^{3} S \rightarrow 2^{3} P$ Transitions.......... 66

16.5. Helium $n^{3} P \rightarrow 2^{3} S$ Transitions............ 67

16.6. Helium $n^{3} D \rightarrow 2^{3} P$ Transitions........... 67

16.7. Excitation of the Helium $n=2$ Levels....... 67

16.8. Thresholds of Helium Excitation Functions. . 70

17. Heavy Rare Gases................... 71

18. Alkali Atoms..................... 72

19. Heavy Two-Electron Systems............ 77

20. Experimental Summary ............... 79

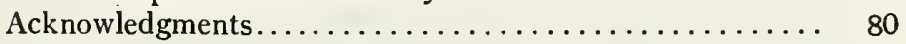

Bibliography.......................... 81

Tables........................... 85

\section{THEORY}

\section{Introduction}

In the first part of this review we direct our attention to the theoretical aspects of the excitation of atoms by electron impact. We examine the atoms in order of increasing complexity beginning with the simplest of all atomic systems, atomic hydrogen, and then in turn consider helium, the heavier inert gases, mercury, the alkali metals, oxygen, and nitrogen, as well as certain positive ions. Because a hydrogen atom has only a single electron, it has been the subject of the most detailed investigations. We therefore begin by developing the theory for atomic hydrogen leaving the generalization to more complex atoms until later. It is important to realize however that even for the case of atomic hydrogen, the work involved in obtaining accurate excitation cross sections is formidable and that indeed no exact solution for the problem of the excitation of the two lowest states of atomic hydrogen, the $2 s$ and $2 p$ states, has yet been obtained. The only method by which we can assess the accuracy of the various approximations which have been employed is by comparing them with each other and with the available experimental data. At the present time the situation continues to be very uncertain and a great deal of investigation, both theoretical and experimental, remains to be done. Our procedure will be to commence with the simplest approximations and to introduce progressively the more involved and, in general, more accurate and revealing approximations. However, before proceeding with this endeavor we draw attention to previous reviews of the theoretical work on the electron impact excitation of atomic systems which include the im- 
portant articles by Massey (1956) and by Peterkop and Veldre (1966).

\section{Hydrogen Atoms}

We consider a system composed of two electrons denoted by 1,2 moving in the Coulomb field of a proton which, to sufficient approximation, we may regard as possessing infinite mass. Then the Schrödinger equation describing this system takes the form

$$
\left[\frac{-\hbar^{2}}{2 m}\left(\nabla_{1}^{2}+\nabla_{2}^{2}\right)-\frac{e^{2}}{r_{1}}-\frac{e^{2}}{r_{2}}+\frac{e^{2}}{r_{12}}-E_{T}\right] \Psi\left(\mathbf{r}_{1}, \mathbf{r}_{2}\right)=0
$$

where $r_{1}, r_{2}$ are the position vectors of electrons 1,2 referred to the proton as origin, $r_{12}$ is the interelectron distance, $E_{T}$ is the total energy of the system, and $\Psi\left(r_{1}, r_{2}\right)$ is the total wave function characterizing the two electrons.

We now expand $\Psi$ in terms of the orthogonal and normalized set of hydrogen atom wave functions $\psi_{n}(r)$ satisfying the equation

$$
\left[\left(-\hbar^{2} / 2 m\right) \nabla^{2}-\left(e^{2} / r\right)-E_{n}\right] \psi_{n}(\mathbf{r})=0,
$$

where the $E_{n}$ are the associated eigenenergies. Setting

$$
\Psi\left(\mathrm{r}_{1}, \mathrm{r}_{2}\right)=\underset{p}{S} F_{p}\left(\mathrm{r}_{2}\right) \psi_{p}\left(\mathrm{r}_{1}\right),
$$

where the symbol $\mathbf{S}$ denotes a summation over the discrete states and an integration over the continuum states, substituting into (1), multiplying across by $\psi_{n}{ }^{*}\left(r_{1}\right)$, and integrating with respect to $r_{1}$, we obtain

$\left[\nabla_{2}{ }^{2}+k_{n}{ }^{2}\right] F_{n}\left(\mathbf{r}_{2}\right)=\frac{2 m}{\hbar^{2}} \int \psi_{n}^{*}\left(\mathbf{r}_{1}\right) V\left(\mathbf{r}_{1}, \mathbf{r}_{2}\right) \Psi\left(\mathbf{r}_{1}, \mathbf{r}_{2}\right) d \mathbf{r}_{1}$,

where

$$
V\left(\mathbf{I}_{1}, \mathbf{I}_{2}\right)=\left(e^{2} / r_{12}\right)-\left(e^{2} / r_{2}\right)
$$

is the interaction energy between electron 2 and a hydrogen atom composed of electron 1 and the proton, and the wave number $k_{n}$ is given by

$$
k_{n}^{2}=\left(2 m / \hbar^{2}\right)\left(E_{r}-E_{n}\right) \text {. }
$$

Let us now suppose that electron 2 impinges upon a hydrogen atom in the ground $1 s$ state which we will denote by 1 , the direction of incidence being given by the wave vector $k_{1}$. Then the asymptotic behavior of the function $F_{n}(r)$ for large $r$ takes the form

$$
F_{n}(\mathbf{r}) \sim \exp \left(i \mathbf{k}_{1} \cdot \mathbf{r}\right) \delta_{n 1}+r^{-1} \exp \left(i k_{n} r\right) f_{n}(\theta, \phi),
$$

where $f_{n}(\theta, \phi)$ is the scattering amplitude corresponding to the excitation of the $n$th state of the hydrogen atom and $\theta, \phi$ are the polar angles of $\mathrm{I}$ referred to the direction of incidence as polar axis.

Introducing the Green's function $G\left(\mathrm{r}, \mathrm{r}_{2}\right)$ for a free particle satisfying the equation

$$
\left(\nabla^{2}+k_{n}^{2}\right) G\left(\mathbf{r}, \mathbf{r}_{2}\right)=\delta\left(\mathbf{r}-\mathbf{r}_{2}\right)
$$

we may write

$$
\begin{aligned}
F_{n}(\mathbf{r})=\exp \left(i \mathbf{k}_{1} \cdot \mathbf{r}\right) \delta_{n 1} & +\frac{2 m}{\hbar^{2}} \iint G\left(\mathbf{r}, \mathbf{r}_{2}\right) \psi_{n}^{*}\left(\mathbf{r}_{1}\right) \\
& \times V\left(\mathbf{r}_{1}, \mathbf{r}_{2}\right) \Psi\left(\mathbf{r}_{1}, \mathbf{r}_{2}\right) d \mathbf{r}_{1} d \mathbf{r}_{2} .
\end{aligned}
$$

In order that $F_{n}$ should have the correct asymptotic form (7) we choose

$$
G\left(\mathbf{r}, \mathbf{r}_{2}\right)=-\exp \left(i k_{n}\left|\mathbf{r}-\mathbf{r}_{2}\right|\right) /\left(4 \pi\left|\mathbf{r}-\mathbf{r}_{2}\right|\right) .
$$

Now for large $r$

$$
k_{n}\left|\mathrm{r}-\mathrm{r}_{2}\right| \sim k_{n} r-\mathrm{k}_{n} \cdot \mathrm{r}_{2},
$$

where $\mathbf{k}_{n}$ is a wave vector in the direction of $\mathbf{r}$, and so we see that

$$
\begin{array}{r}
f_{n}(\theta, \phi)=-(4 \pi)^{-1} \frac{2 m}{\hbar^{2}} \iint \exp \left(-i \mathbf{k}_{n} \cdot \mathbf{r}_{2}\right) \psi_{n} *\left(\mathbf{r}_{1}\right) \\
\times V\left(\mathbf{r}_{1}, \mathbf{r}_{2}\right) \Psi\left(\mathbf{r}_{1}, \mathbf{r}_{2}\right) d \mathbf{r}_{1} d \mathbf{r}_{2} .
\end{array}
$$

The differential cross section for the excitation of the $n$th state of a hydrogen atom is given in terms of the scattering amplitude $f_{n}(\theta, \phi)$, corresponding to incident electrons scattered through angles $\theta, \phi$, by the formula

$$
I_{n}(\theta, \phi)=\left(k_{n} / k_{1}\right)\left|f_{n}(\theta, \phi)\right|^{2}
$$

while the total cross section takes the form

$$
Q_{n}=\frac{k_{n}}{k_{1}} \iint\left|f_{n}(\theta, \phi)\right|^{2} \sin \theta d \theta d \phi
$$

\subsection{First Born Approximation}

We now make the simplest of all approximations, the first Born approximation, which depends upon the assumption that the incident electron interacts only slightly with the target atom so that its wave function may be closely approximated by the plane wave $\exp \left(i k_{1} \cdot r\right)$ which would be the correct function in the absence of all interaction. We may anticipate that this approximation should be valid when the speed of the incident electron is great in comparison with that of the electron in the target atom. This is essentially equivalent to the requirement that $k_{1} a \gg 1$, where $a$ is the range of the electron-atom interaction. Under these circumstances we may substitute

$$
\Psi\left(r_{1}, r_{2}\right) \cong \exp \left(i k_{1} \cdot r_{2}\right) \psi_{1}\left(r_{1}\right)
$$

into the right-hand side of (12). This yields the first Born approximation to the scattering amplitude

$$
f_{n}(\theta, \phi)=-(4 \pi)^{-1} \frac{2 m}{\hbar^{2}} \int \exp \left\{i\left(\mathbf{k}_{1}-\mathbf{k}_{n}\right) \cdot \mathbf{r}_{2}\right\} V_{n \mathbf{1}}\left(\mathbf{r}_{2}\right) d \mathbf{r}_{2}
$$


where

$$
V_{n 1}\left(\mathbf{r}_{2}\right)=\int \psi_{n}^{*}\left(\mathbf{r}_{1}\right) V\left(\mathbf{r}_{1}, \mathbf{r}_{2}\right) \psi_{1}\left(\mathbf{r}_{1}\right) d \mathbf{r}_{1} .
$$

Aithough the application of this approximation can only be justified at high impact energies, it appears to yield quite useful results even for electron energies which are fairly close to the threshold energy for excitation.

Denoting the momentum change of the incident electron by the vector $\hbar \mathrm{K}$, where $\mathrm{K}=\mathbf{k}_{1}-\mathbf{k}_{n}$, and using Bethe's integral

$$
\int \frac{\exp \left(i \mathbf{K} \cdot \mathbf{r}_{2}\right)}{\left|\mathbf{r}_{1}-\mathbf{r}_{2}\right|} d \mathbf{r}_{2}=\frac{4 \pi}{K^{2}} \exp \left(i \mathbf{K} \cdot \mathbf{r}_{1}\right),
$$

we may express the scattering amplitude in the form

$$
f_{n}(K)=-\left(2 m e^{2} / \hbar^{2} K^{2}\right) g(1-n) \quad(n \neq 1),
$$

where

$$
g(1-n)=\int \psi_{n}{ }^{*}(\mathbf{r}) \exp (i \mathbf{K} \cdot \mathbf{r}) \psi_{\mathbf{1}}(\mathbf{r}) d \mathbf{r},
$$

the term arising from the electron-proton interaction vanishing here because of the orthogonality of the atomic wave functions.

We see from formulas (13), (19), and (20) that $\left(k_{1} / k_{n}\right) I_{n}(\theta, \phi)$ depends only on $K$ in the Born approximation, a result which has been verified experimentally by Lassettre and his collaborators for the $2^{1} S$ and $2^{1} P$ excitations of helium (Silverman and Lassettre, 1964; Lassettre and Jones, 1964; Lassettre, Krasnow, and Silverman, 1964).

It is sometimes useful to write the differential cross section in terms of a generalized oscillator strength

$$
\hat{l}_{1 n}(K)=\left(2 m / \hbar^{2}\right)\left[\left(E_{1}-E_{n}\right) / K^{2}\right]|g(1-n)|^{2}
$$

which can be shown to satisfy the sum rule

$$
\sum_{n} i_{1 n}(K)=1 \text {. }
$$

As the momentum change $\hbar \mathrm{K}$ tends to zero, $\int_{1 n}(K)$ approaches the optical oscillator strength given by

$$
\frac{2 m}{\hbar^{2}}\left(E_{1}-E_{n}\right)\left|\int \psi_{n}(\mathbf{r}) z \psi_{1}(\mathbf{r}) d \mathbf{r}\right|^{2},
$$

where the $z$ coordinate of the atomic electron is measured in the direction of $\mathbf{K}$.

Now

and so

$$
K^{2}=k_{1}{ }^{2}+k_{n}^{2}-2 k_{1} k_{n} \cos \theta
$$

$$
K d K=k_{1} k_{n} \sin \theta d \theta
$$

from which it follows that

$$
\begin{aligned}
I_{n}(\theta, \phi) \sin \theta d \theta & =k_{1}^{-2}\left|f_{n}(K)\right|^{2} K d K \\
& =\frac{2 m e^{4}}{\hbar^{2} k_{1}^{2}} \frac{f_{1 n}(K)}{E_{1}-E_{n}} \frac{d K}{K} .
\end{aligned}
$$

The total cross section for the excitation of the $n$th state of hydrogen may be written in the form

$$
Q_{n}=\frac{2 \pi}{k_{1}^{2}} \int_{K_{\mathrm{m} \text { in }}}^{K_{\mathrm{max}}}\left|f_{n}(K)\right|^{2} K d K,
$$

where $K_{\min }=k_{1}-k_{n}$ and $K_{\max }=k_{1}+k_{n}$. Since

$$
k_{1}^{2}-k_{n}{ }^{2}=\left(2 m / \hbar^{2}\right)\left(E_{n}-E_{1}\right)
$$

by the conservation of energy, we see that for high electron impact energies

$$
K_{\min } \cong\left(m / \hbar^{2} k_{1}\right)\left(E_{n}-E_{1}\right), \quad K_{\max } \cong 2 k_{1} .
$$

As illustrative examples let us now consider the excitation of the $2 s$ and $2 p$ states of atomic hydrogen from the ground $1 s$ state. It can be readily shown that

and

$$
\left|f_{28}(K)\right|^{2}=128 a_{0}^{2} /\left(K^{2} a_{0}^{2}+\frac{9}{4}\right)^{6}
$$

$$
\left|f_{2 p m}(K)\right|^{2}=288 \delta_{0 m} / K^{2}\left(K^{2} a_{0}^{2}+\frac{9}{4}\right)^{6},
$$

where $m$ is the magnetic quantum number of the $2 p$ state referred to the momentum change vector $\hbar \mathbf{K}$ as polar axis. We see at once that according to the first Born approximation the cross sections vanish for the excitation of $2 p$ states with magnetic quantum numbers $m= \pm 1$ referred to the direction of the momentum change vector $\hbar \mathrm{K}$ as polar axis. We also note that for the $1 s \rightarrow 2 s$ excitation the differential cross section is weakly dependent upon $K$ for small angles of scattering while for the $1 s \rightarrow 2 p 0$ excitation it behaves like $K^{-2}$. For large angles of scattering the differential cross sections for the $1 s \rightarrow 2 s$ and $1 s \rightarrow 2 p 0$ excitations decay like $K^{-12}$ and $K^{-14}$, respectively; these are much more rapid decreases with angle than for elastic scattering which falls off like $K^{-4}$. The differential cross sections for the $1 s \rightarrow 2 s$ and $1 s \rightarrow 2 p$ excitations at an electron impact energy of $100 \mathrm{eV}$ calculated according to the first Born approximation are illustrated in Fig. 1.

Substituting expressions (28) and (29) into (25) and performing the integrations over $K$ yields at high impact energies

and

$$
Q_{2 s} \sim 128\left(\frac{2}{3}\right)^{10} \pi / 5 k_{1}{ }^{2}
$$

$$
Q_{2 p} \sim\left[256\left(\frac{2}{3}\right)^{10} \pi / k_{1}^{2}\right] \ln \left(4 a_{0} k_{1}\right),
$$

where $k_{1}$ is the wave number of the incident electron, from which it follows that the total cross sections for the $1 s \rightarrow 2 s$ and $1 s \rightarrow 2 p$ excitations decay as $E^{-1}$ and $E^{-1} \ln E$, respectively, for high energies $E$ of impact. The total cross sections for the $1 s \rightarrow 2 s$ and $1 s \rightarrow 2 p$ excitations of atomic hydrogen calculated according to the first Born approximation are displayed in Fig. 2. We see that the cross section for the $1 s \rightarrow 2 p$ cxcitation, which corresponds to an optically allowed transition, is considerably larger than the cross section for the $1 s \rightarrow 2 s$ excitation, which is associated with an optically forbidden transition. In addition the peak of the $1 s \rightarrow 2 p$ excitation cross section occurs at a higher energy than that for the $1 s \rightarrow 2 s$ excitation, and decays less rapidly with in- 
creasing energy, as was already quite clear from the asymptotic behavior of the cross sections given by formulas (30) and (31).

It is also instructive to make a partial wave analysis of the total excitation cross section:

$$
Q_{n}=\sum_{\ell=0}^{\infty} Q_{n}^{\ell}=\sum_{\ell=0}^{\infty} \sum_{\ell^{\prime}=0}^{\infty} Q_{n}^{\ell \ell \prime},
$$

where the partial cross section $Q_{n}{ }^{\prime \prime \prime}$ is the contribution to the total cross section arising from incident electrons having azimuthal quantum number $\ell$ and scattered electrons having quantum number $\ell^{\prime}$, that is incident and scattered electrons with angular momenta $\hbar[\ell(\ell+1)]^{1 / 2}$ and $\hbar\left[\ell^{\prime}\left(\ell^{\prime}+1\right)\right]^{1 / 2}$, respectively, while

$$
Q_{n}^{\ell}=\sum_{\ell \prime=0}^{\infty} Q_{n}^{\ell \ell \prime}
$$

is the partial cross section corresponding to incident electrons having angular momentum given by the quantum number $\ell$ and scattered electrons having all possible values of the angular momentum. For $s \rightarrow s$ transitions the only nonvanishing partial cross sections are those for which $\ell=\ell^{\prime}$, while for $s \rightarrow p$ transitions only the partial cross sections $Q_{n}^{\ell, \ell-1}$ and $Q_{n}^{\ell, \ell+1}$ are nonzero. Since

$$
\exp i \mathbf{k} \cdot \mathbf{r}=\sum_{\ell=0}^{\infty}(2 \ell+1) i^{\ell} j_{\ell}(k r) P_{\ell}(\cos \theta),
$$

where $j_{\ell}(\rho)$ is a spherical Bessel function, it follows that for $s \rightarrow s$ transitions the first Born approximation to the $\ell$ th-order partial cross section is given by

where

$$
Q_{n}^{\ell}=4 \pi\left(k_{n} / k_{1}\right)(2 \ell+1)\left(q_{n}^{\ell}\right)^{2},
$$

$$
q_{n}^{\ell}=\frac{2 m}{\hbar^{2}} \int_{0}^{\infty} j_{\ell}\left(k_{1} r\right) j_{\ell}\left(k_{n} r\right) V_{n 1}(r) r^{2} d r
$$

A partial wave analysis has been carried out for the $1 s \rightarrow 2 s$ and $1 s \rightarrow 3 s$ excitations (Bates and Miskelly, $1957)$ and the $1 s \rightarrow 2 p$ excitation (Vainshtein, 1961) of hydrogen atoms using the first Born approximation. The partial cross sections $Q_{n}{ }^{\ell}$ for the $1 s \rightarrow 2 s$ and $1 s \rightarrow 2 p$

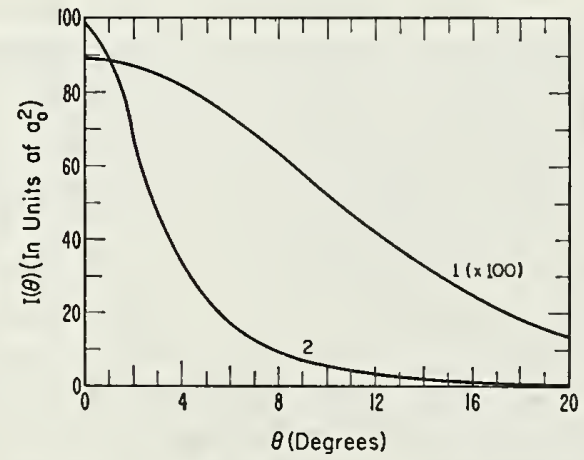

FIG. 1. Differential cross sections for the excitation of atomic hydrogen at $100-\mathrm{eV}$ electron impact energy, calculated using the frst Born approximation. Curve $1 ; 1 s \rightarrow 2 s$, Curve $2: 1 s \rightarrow 2 p$.

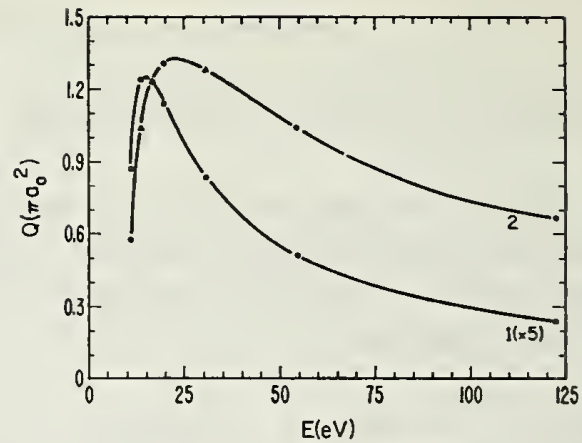

FIg. 2. Total cross sections for the excitation of atomic hydrogen, calculated using the first Born approximation (Omidvar, 1964). Curve $1: 1 s \rightarrow 2 s$, Curve $2: 1 s \rightarrow 2 p$.

excitations are displayed in Figs. 3 and 4, respectively. It can be readily seen that at a given impact energy many more partial waves make a significant contribution to the total $1 s \rightarrow 2 p$ excitation cross section than to the $1 s \rightarrow 2 s$ cross section.

The contrasting behavior of the $1 s \rightarrow 2 s$ and $1 s \rightarrow 2 p$ excitation cross sections arises from the fact that their respective interaction potentials have differing asymptotic forms for large radial distances $r$. Thus $V_{2 s, 18}$ decays exponentially with increasing $r$ while $V_{2 p, 1 s}$ falls off much more slowly with an $r^{-2}$ dependence for large $r$. This provides the explanation for the different angular distributions given by (28) and (29), the different high-energy behaviors given by (30) and (31), and the different convergences of the partial wave expansions illustrated in. Figs. 3 and 4.

The first Born approximation has been employed by a number of investigators to calculate the total excitation cross sections for various transitions connecting different states of hydrogen. Suppose that $n^{\prime}, l^{\prime}, m^{\prime}$ and $n, l, m$ are the principal, azimuthal, and magnetic quantum numbers of the initial and final states of the hydrogen atom, respectively, and let us denote the excitation cross section for the transition $n^{\prime} l^{\prime} m^{\prime} \rightarrow n l m$ by $Q_{n^{\prime} l^{\prime} m^{\prime}, n l m}$. We are mainly concerned with the average of this cross section over $m^{\prime}$ and its sum over $m$, that is with the excitation cross section for the transition $n^{\prime} l^{\prime} \rightarrow n l$ given by

$$
Q_{n^{\prime} l^{\prime}, n l}=\left(2 l^{\prime}+1\right)^{-1} \sum_{m^{\prime}} \sum_{m} Q_{n^{\prime} l^{\prime} m^{\prime}, n l m} .
$$

Occasionally we require the excitation cross section for the transition $n^{\prime} \rightarrow n$ between the levels with principal quantum numbers $n^{\prime}$ and $n$; this is obtained by averaging over $l^{\prime}$ and summing over $l$ to yield

$$
\begin{aligned}
Q_{n^{\prime}, n} & =\left(1 / n^{\prime}\right) \sum_{l^{\prime}} \sum_{l} Q_{n^{\prime} l^{\prime}, n l} \\
& =\left(1 / n^{\prime 2}\right) \sum_{l^{\prime}} \sum_{l}\left(2 l^{\prime}+1\right) Q_{n^{\prime} l^{\prime}, n l} .
\end{aligned}
$$

In Tables I-III are presented the values of the excitation cross sections calculated by Vainshtein (1965) for the $n^{\prime} s \rightarrow n s, n^{\prime} s \rightarrow n p$, and $n^{\prime} s \rightarrow n d$ transitions of atomic hydrogen with $n^{\prime}<n$ and $n^{\prime}, n=1, \cdots, 9$. For the sake 


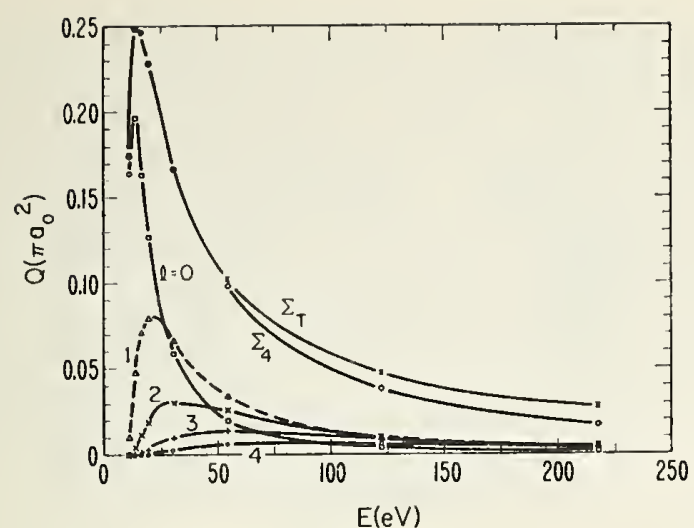

FIG. 3. Partial cross sections $Q_{2} \&$ for the $1 s \rightarrow 2 s$ excitation of atomic hydrogen, calculated using the first Born approximation (Omidvar, 1964). $\Sigma_{4}$ denotes

$\sum_{\ell=0}^{4} Q_{2 s}^{\ell}$,

$\Sigma_{T}$ denotes

$$
\sum_{\ell=0}^{\infty} Q_{2 s}^{\ell}
$$

of convenience the energy of the incident electron is given in threshold units, that is, in ternıs of the quantity

$$
\epsilon=E /\left(E_{n}-E_{n^{\prime}}\right)=k_{n^{\prime}}{ }^{2} / \Delta E_{n n^{\prime}}
$$

where $\Delta E_{n n^{\prime}}$ is the excitation energy in rydberg units and $k_{n}$ is the wave number of the incident electron.

$V$ eldre and Rabik (1965) have calculated cross sections for the $1 s \rightarrow n s, 1 s \rightarrow n p$, and $n s \rightarrow(n+1) s$ excitations of atomic hydrogen using the first Born approximation. Their excitation cross-section curves together with those for the lower values of $n$ derived from the calculations of Vainshtein (1965) are displayed in Figs. 5-7, respectively. Inspection of these figures reveals that according to the first Born approximation the value $\epsilon_{M}$ of $\epsilon$ for which the cross section attains its maximum value is insensitive to $n$ for both the $1 s \rightarrow n s$ and the $1 s \rightarrow n p$ excitations, although for the $n s \rightarrow$ $(n+1) s$ excitations $\epsilon_{M}$ increases significantly with increasing $n$. Further we note that for each set of transitions, the smaller the value of $\epsilon_{M}$, the more gradually does the excitation cross-section decay with increasing $\epsilon$.

Calculations of the excitation cross sections for a number of transitions in atomic hydrogen have been also carried out by Milford and his collaborators (McCoyd, Milford, and Wahl, 1960; Fisher, Milford, and Pomilla, 1960; Milford, Morrissey, and Scanlon, 1960) using the first Born approximation. They investigated all transitions between states with principal quantum numbers $n^{\prime}=3$ and $n=4$; all optically allowed transitions between states with $n^{\prime}=3$ and $n=5$, between states with $n^{\prime}=4$ and $n=5$, and between states with $n^{\prime}=5$ and $n=6$; together with a few other transitions. Some of their excitation cross section curves are displayed in Figs. 8-10.

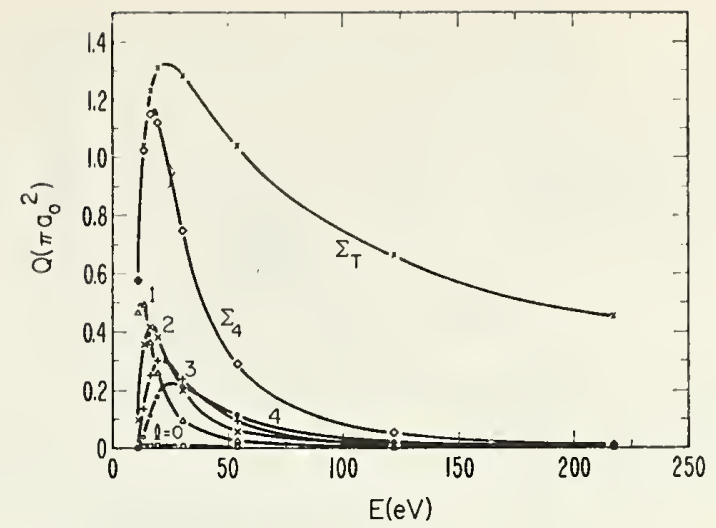

FIG. 4. Partial cross sections $Q_{2 p}^{\ell}$ for the $1 s \rightarrow 2 p$ excitation of atomic hydrogen, calculated using the first Born approximation (Omidvar, 1964). $\Sigma_{4}$ denotes

$$
\sum_{\ell=0}^{4} Q_{2 p}^{\ell}
$$

$\Sigma_{T}$ denotes

$$
\sum_{\ell=0}^{\infty} Q_{2 p}^{\ell}
$$

(a)

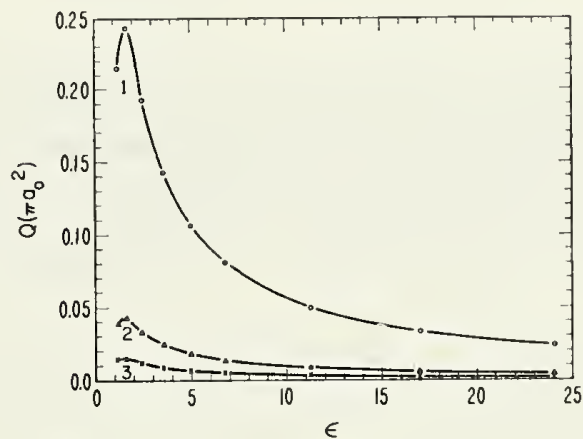

(b)

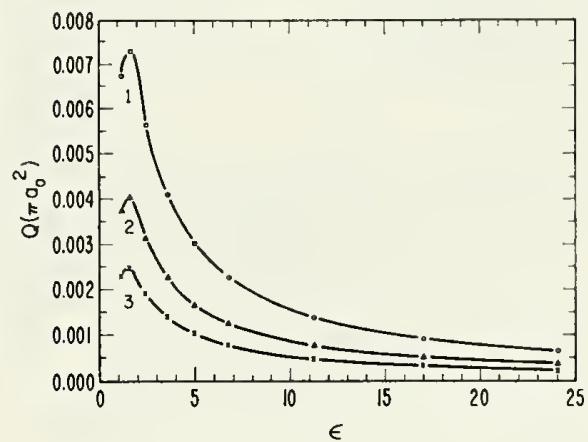

(c)

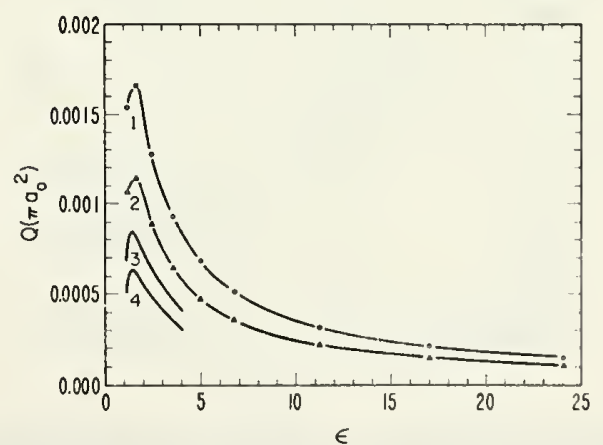

FIG. 5. Total cross sections for the $1 s \rightarrow n s$ excitations of atomic hydrogen for $n=2, \cdots, 11$, calculated using the first Born approximation [ $\epsilon$ is electron energy in threshold units defined by Eq. (38) ] (a) Curve 1: $1 s \rightarrow 2 s$, Curve $2: 1 s \rightarrow 3 s$, Curve $3: 1 s \rightarrow 4 s$. (b) Curve 1: $1 s \rightarrow 5 s$, Curve 2: $1 s \rightarrow 6 s$, Curve $3: 1 s \rightarrow 7 s$. (c) Curve $1 ; 1 s \rightarrow 8 s$, Curve $2: 1 s \rightarrow 9 s$, Curve $3 ; 1 s \rightarrow 10 s$, Curve $4: 1 s \rightarrow 11 s$. 
(a)

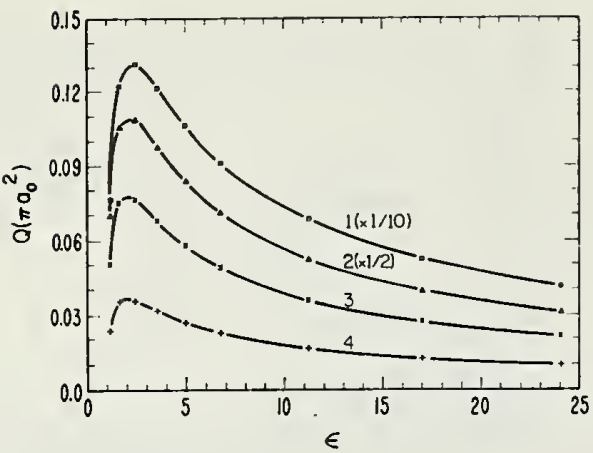

(b)

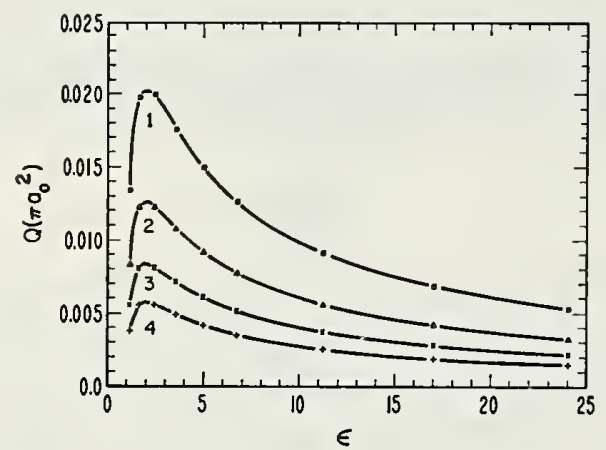

Fig 6. Total cross sections for the $1 s \rightarrow n p$ excitations of atomic hydrogen for $n=2, \cdots, 9$, calculated using the first Born approximation [ $\epsilon$ is electron energy in threshold units defined by Eq. (38) ] (Vainshtein, 1965; Veldre and Rabik, 1965). (a) Curve 1: $1 s \rightarrow 2 p$, Curve 2: $1 s \rightarrow 3 p$, Curve 3: $1 s \rightarrow 4 p$, Curve $4: 1 s \rightarrow 5 p$. (b) Curve 1: $1 s \rightarrow 6 p$, Curve 2: $1 s \rightarrow 7 p$, Curve $3: 1 s \rightarrow 8 p$, Curve 4: $1 s \rightarrow 9 p$.

(a)

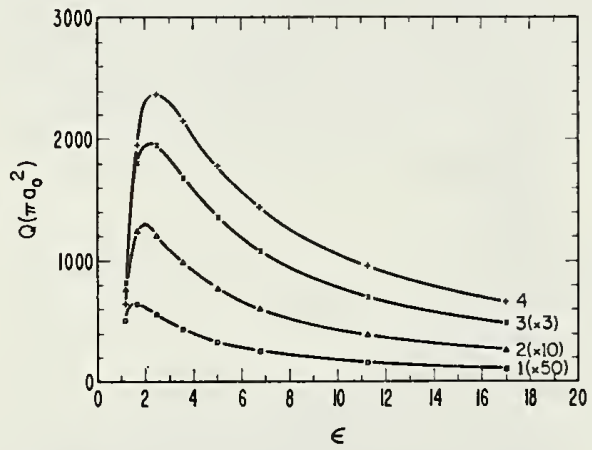

(b)

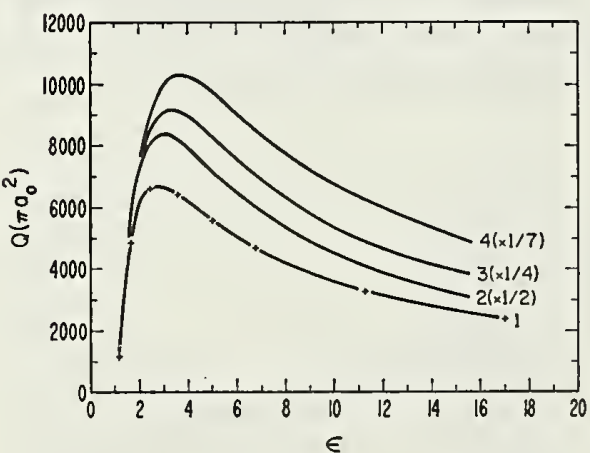

FIg. 7. Total cross sections for the $n s \rightarrow(n+1) s$ excitations of atomic hydrogen for $n=2, \cdots, 9$, calculated using the first Born approximation $[\epsilon$ is electron energy in threshold units defined by Eq. (38) ] (Vainshtein, 1965; Veldre and Rabik, 1965). (a) Curve 1: $2 s \rightarrow 3 s$, Curve 2: $3 s \rightarrow 4 s$, Curve $3: 4 s \rightarrow 5 s$, Curve 4: $5 s \rightarrow 6 s$. (b) Curve 1: $6 s \rightarrow 7 s$, Curve 2: $7 s \rightarrow 8 s$, Curve 3: $8 s \rightarrow 9 s$, Curve $4: 9 s \rightarrow 10 s$.

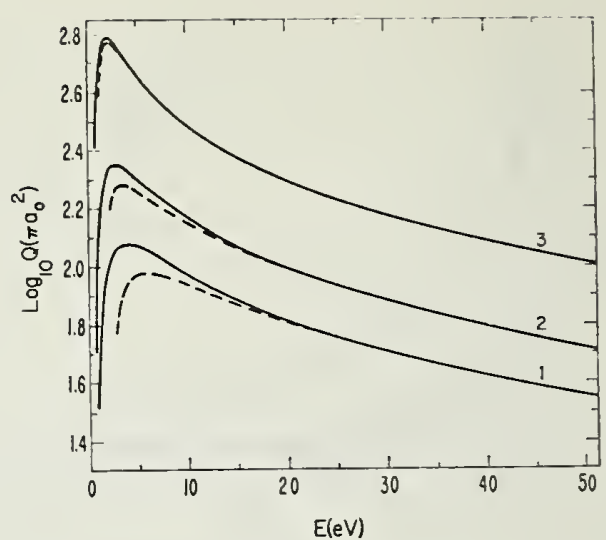

FIG. 8. Total cross sections for the excitation of atomic hydrogen, calculated using the first Born approximation and the Bethe approximation (McCoyd, Milford, and Wahl, 1960). Curve 1: $3 s \rightarrow 4 p$, Curve 2: $3 p \rightarrow 4 d$, Curve $3: 3 d \rightarrow 4 f$. Solid curves denote first Born approximation while dashed curves denote Bethe approximation.

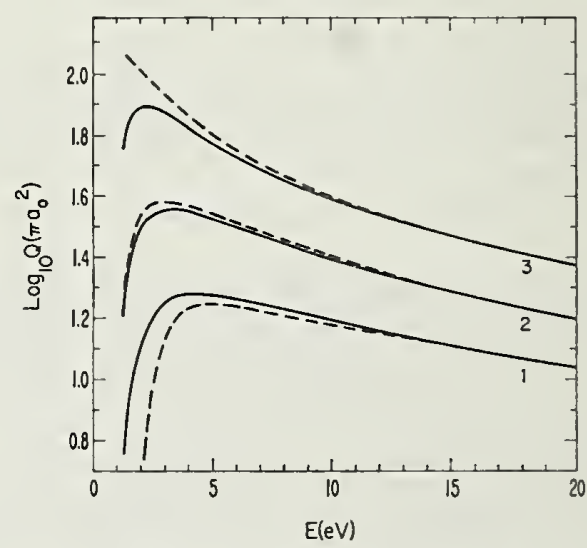

FIG. 9. Total cross sections for the excitation of atomic hydrogen, calculated using the first Born approximation and the Bethe approximation (McCoyd, Milford, and Wahl, 1960). Curve 1: $3 s \rightarrow 5 p$, Curve $2: 3 p \rightarrow 5 d$, Curve $3: 3 d \rightarrow 5 f$. Solid curves denote first Born approximation while dashed curves denote Bethe approximation.

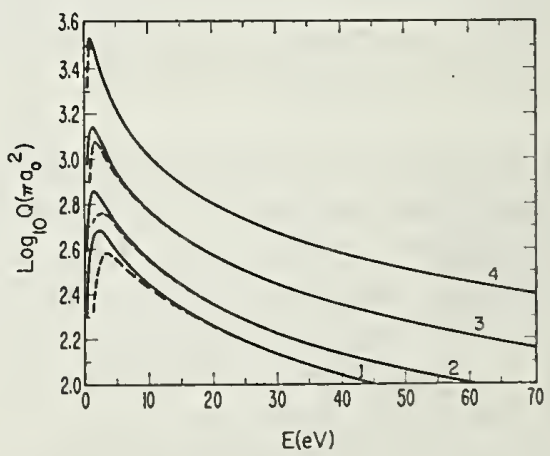

Fig. 10. Total cross sections for the excitation of atomic hydrogen, calculated using the first Born approximation and the Bethe approximation (Fisher, Milford, and Pomilla, 1960). Curve 1: $4 s \rightarrow 5 p$, Curve $2: 4 p \rightarrow 5 d$, Curve $3: 4 d \rightarrow 5 f$, Curve $4: 4 f \rightarrow 5 g$. Solid curves denote first Born approximation while dashed curves denote Bethe approximation. 
Detailed calculations have also been performed on the excitation of various levels of atomic hydrogen. (McCarroll, 1957; Boyd, 1959; McCrea and McKirgan, 1960; Omidvar, 1965; Kingston and Lauer, 1966a). Cross sections for the transitions $1 s \rightarrow n(n=2, \cdots, 10)$, $2 s \rightarrow n, 2 p 0 \rightarrow n, 2 p \pm 1 \rightarrow n(n=3, \cdots, 10)$, and $3 \rightarrow n$ $(n=4, \cdots, 8)$ are given in Tables IV-VIII.

The eigenenergy $E_{n}$ of the $n$th level of a hydrogen atom is $-1 / n^{2}$ rydbergs so that for large values of the principal quantum number $n$ we have

$$
d n=\frac{1}{2} n^{3} d E_{n} .
$$

Hence the cross section for the excitation of atomic hydrogen to all energy levels within the range $E_{n}$ to $E_{n}+d E_{n}$ rydbergs is given by

$$
\frac{1}{2} n^{3} Q_{n} d E_{n} .
$$

If $Q_{i}\left(E^{\prime}\right) d E^{\prime}$ denotes the cross section for the ejection of an electron into the continuum with energy between $E^{\prime}$ and $E^{\prime}+d E^{\prime}$ rydbergs, it follows that $\frac{1}{2} n^{3} Q_{n}$ regarded as a function of $E_{n}$ is continuous with $Q_{i}\left(E^{\prime}\right)$. In Fig. 11 we illustrate this continuity for transitions emanating from the ground state of atomic hydrogen (McCarroll, 1957).

\subsection{Bethe Approximation}

A simplification of the first Born approximation due to Bethe can be readily derived by expanding $\exp (i \mathbf{K} \cdot \mathbf{r})$ in the formula (20). Choosing the $z$ axis along the momentum change vector $\hbar \mathrm{K}$, which is therefore the direction of quantization of the atomic wave functions, yields

$$
\begin{aligned}
g(1-n l m)= & \delta_{0 m} \int \psi_{n l 0} *(\mathbf{r}) \psi_{1}(\mathrm{r}) \\
& \times\left(1+i K z-\frac{1}{2} K^{2} z^{2}+\cdots\right) d \mathrm{r}
\end{aligned}
$$

where $\psi_{n l 0}$ is the wave function of the state of the hydrogen atom with principal and azimuthal quantum numbers $n, l$ and magnetic quantum number $m=0$. Setting

$$
\left(z^{8}\right)_{1, n l}=\int \psi_{n l 0} *(\mathrm{r}) z^{s} \psi_{1}(\mathrm{r}) d \mathrm{r}
$$

we obtain

$$
g(1-n l 0)=i K(z)_{1, n l}-\frac{1}{2} K^{2}\left(z^{2}\right)_{1, n l}+\cdots
$$

and so

$$
\begin{aligned}
\left|f_{1, n l m}(K)\right|^{2}=\left(4 \delta_{0 m} / K^{2} a_{0}^{2}\right) \mid & (z)_{1, n l} \\
& +\frac{1}{2}(i K)\left(z^{2}\right)_{1, n l}+\left.\cdots\right|^{2}
\end{aligned}
$$

which converges provided $K a_{0} \ll 1$ since $a_{0}$ is the approximate range of the atomic wave functions. For $K a_{0} \gg 1$ we note that $\left|f_{1, n 10}(K)\right|^{2}$ provides a negligible contribution to the excitation cross section $Q_{n l}$.

Let us first consider optically allowed transitions for which the final state is a $p$ state so that $l=1$. Then $(z)_{1, n l}$ is nonvanishing and we may put

$\left|f_{1, n l m}(K)\right|^{2} \cong\left(4 \delta_{0 m} / K^{2} a_{0}^{2}\right)\left|(z)_{1, n l}\right|^{2} \quad(l=1)$.

Hence integrating from $K_{\min }=\left(m / h^{2} k_{1}\right)\left(E_{n}-E_{1}\right)$ to $K_{\max }=2 k_{1}$ we find that

$$
Q_{n l} \cong \frac{8 \pi\left|(z)_{1, n l}\right|^{2}}{k_{1}^{2} a_{0}^{2}} \ln \frac{2 \hbar^{2} k_{1}^{2}}{m\left(E_{n}-E_{1}\right)} \quad(l=1) .
$$

Thus we see that the $1 s \rightarrow n p$ excitation cross section decays as $E^{-1} \ln E$ for large values of the impact energy $E=\hbar^{2} k_{1}{ }^{2} / 2 m$ in accordance with the Born approximation result obtained previously.

For optically forbidden transitions $(z)_{1, n l}=0$. If however $\left(z^{2}\right)_{1, n l}$ does not vanish we can write

$$
\left|f_{1, n l m}(K)\right|^{2} \cong\left(\delta_{0 m} / a_{0}^{2}\right)\left|\left(z^{2}\right)_{1, n l}\right|^{2} .
$$

Neglecting the contribution from $K a_{0}>1$ and using expression (45) for $K a_{0} \leqq 1$ we obtain

$$
Q_{n} \cong\left(\pi / k_{1}^{2} a_{0}^{2}\right)\left[\left|\left(z^{2}\right)_{1, n l}\right|^{2} / a_{0}^{2}\right]
$$

so that the excitation cross section for a monopole transition $1 s \rightarrow n s$ or a quadrupole transition $1 s \rightarrow n d$ decays like $E^{-1}$ for large values of $E$ as found before.

The Bethe approximation may also be derived by replacing the interaction energy $V\left(\mathbf{r}_{1}, \mathbf{r}_{2}\right)$ by its asymptotic form for large radial distances $r_{2}$ of the incident electron. In the case of $1 s \rightarrow n l m$ transitions this can be achieved by substituting $\left(r_{1}^{l} / r_{2}^{l+1}\right) P_{l}\left(\cos \theta_{12}\right)$ for $1 / r_{12}$ in formula (5), where $\theta_{12}$ is the angle between $r_{1}$ and $r_{2}$, and then evaluating the scattering amplitude by using formulas (16) and (17). This approach yields the same expression for $f_{1, n l m}$ as the method described previously except when $l=0$ for which case it gives a vanishing result.

The upper limit of the $K$ integration in formula (25) may be replaced by an adjustable parameter $K_{c}$ which is chosen so as to produce agreement with the first Born approximation at high electron impact energies. Calculations along these lines have been performed by Milford and his associates (McCoyd, Milford, and

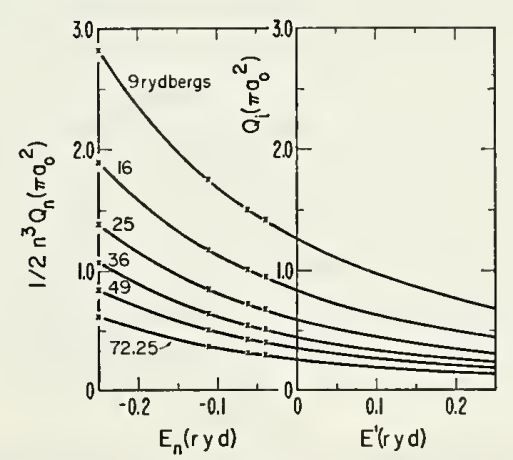

FIG. 11. Illustration of the continuity of $\frac{1}{2} n^{8} Q_{n}$ as a function of $E_{n}$ with $Q_{i}$ as a function of $E^{\prime}$ (McCarroll, 1957). $E_{n}$ is the (negative) energy of an excited electron in level $n$; $E^{\prime}$ is the (positive) energy of an ejected electron in the continuum. The crosses refer to levels with $n=2,3,4,5$. The numbers on the curves are the incident electron energies in rydbergs. 
Wahl, 1960; Fisher, Milford, and Pomilla, 1960; Milford, Morrissey, and Scanlon, 1960; McCoyd and Milford, 1963; Milford, 1960; Scanlon and Milford, 1961), their Bethe approximation cross-section curves being compared with those obtained using the first Born approximation in Figs. 8-10. Inspection of these figures shows that the Bethe formula is in reasonably satisfactory accord with the first Born approximation down to electron impact energies which are not greatly above the energy where the Born cross section attains its maximum value. Since the Born approximation itself ceases to be valid below this energy, the Bethe approximation provides a valuable alternative formula for determining excitation cross sections as long as it is not employed at low impact energies.

McCoyd and Milford (1963) have used the best available values of the cutoff parameter $K_{c}$ to determine the constants $C_{n^{\prime} l^{\prime}, n l}$ and $D_{n^{\prime} l^{\prime}, n l}$ in the Bethe formula for the excitation cross section

$$
Q_{n^{\prime} l^{\prime}, n l}=\left(C_{n^{\prime} l^{\prime}, n l} / E\right) \ln \left(D_{n^{\prime} l^{\prime}, n l} E\right)
$$

for all transitions satisfying $n-n^{\prime}=1$ or 2 and $l-l^{\prime}=1$ with $1 \leq n^{\prime} \leq 10$. The values of these constants when the impact energy $E$ is given in $\mathrm{eV}$ are displayed in Tables IX and X. An extension of these calculations has been made by Kingston and Lauer (1966a; 1966b) who investigated the case $n-n^{\prime}=1$ and 2 for $1 \leq n^{\prime} \leq 6$ and all permissible values of $l^{\prime}$ and $l$. They also evaluated the excitation cross section $Q_{n^{\prime}, n}$ for transitions between the levels with principal quantum numbers $n^{\prime}$ and $n$. As long as the impact energy is not too low this may be satisfactorily approximated by the formula

$$
Q_{n^{\prime}, n}=\left(C_{n^{\prime}, n} / E\right) \ln \left(D_{n^{\prime}, n} E\right),
$$

the values of the constants $C_{n^{\prime}, n}$ and $D_{n^{\prime}, n}$ calculated by Kingston and Lauer $(1966 \mathrm{a} ; 1966 \mathrm{~b})$ being displayed in Table XI.

\subsection{Second Born Approximation}

The first Born approximation neglects coupling to all states other than the initial state and the final state of the transition. One method of allowing for coupling to other states is to employ the second Born approximation to the scattering amplitude. This approximation can be derived in the following manner. We consider the case of the excitation of the $n$th state of atomic hydrogen from the ground state and insert expression (15) for $\Psi$ into the right-hand side of Eq. (9). Then we find that to the first order in the interaction energy $V$ :

$$
\begin{array}{r}
F_{n}(\mathbf{r})=\exp \left(i \mathbf{k}_{1} \cdot \mathbf{r}\right) \delta_{n 1}+\frac{2 m}{\hbar^{2}} \iint G\left(\mathbf{r}, \mathbf{r}_{2}\right) \psi_{n} *\left(\mathbf{r}_{1}\right) \\
\times V\left(\mathbf{r}_{1}, \mathbf{r}_{2}\right) \psi_{1}\left(\mathbf{r}_{1}\right) \exp \left(i \mathbf{k}_{1} \cdot \mathbf{r}_{2}\right) d \mathbf{r}_{1} d \mathbf{r}_{2} .
\end{array}
$$

Now expanding $\Psi$ according to (3), using formula (49) for $F_{n}$ and substituting into the right-hand side of ex- pression (12), we see that to the second order in $V$ :

$$
f_{n}(\theta, \phi)=f_{n}{ }^{(1)}(\theta, \phi)+f_{n}{ }^{(2)}(\theta, \phi),
$$

where $f_{n}{ }^{(1)}$ is just the first Born approximation to the scattering amplitude given by (16) and $f_{n}{ }^{(2)}$ is given by the formula

$$
\begin{gathered}
f_{n}{ }^{(2)}(\theta, \phi)=(4 \pi)^{-1}\left(\frac{2 m}{\hbar^{2}}\right)^{2} S \iint \exp \left\{i\left(\mathbf{k}_{1} \cdot \mathbf{r}_{2}{ }^{\prime}-\mathbf{k}_{n} \cdot \mathbf{r}_{2}\right)\right\} \\
\times \frac{\exp \left(i k_{p}\left|\mathbf{r}_{2}-\mathbf{r}_{2}{ }^{\prime}\right|\right)}{4 \pi\left|\mathbf{r}_{2}-\mathbf{r}_{2}{ }^{\prime}\right|} V_{n p}\left(\mathbf{r}_{2}\right) V_{p 1}\left(\mathbf{r}_{2}{ }^{\prime}\right) d \mathbf{r}_{2} d \mathbf{r}_{2}{ }^{\prime} .
\end{gathered}
$$

Expression (50) is known as the second Born approximation to the scattering amplitude. We see from formula (51) for $f_{n}{ }^{(2)}$ that it involves a summation over all the states of hydrogen including the continuum states. This makes it very difficult to evaluate exactly. However it can be simplified by setting $k_{p}=k$ for all $p$, where $k$ is independent of $p$, in which case the summation can be performed by using the closure relation

$$
\underset{p}{S} \psi_{p}^{*}\left(\mathrm{r}_{1}\right) \psi_{p}\left(\mathrm{r}_{1}{ }^{\prime}\right)=\delta\left(\mathrm{r}_{1}-\mathrm{r}_{1}{ }^{\prime}\right) .
$$

It leads to the result

$$
\begin{aligned}
f_{n}^{(2)}(\theta, \phi) & =(4 \pi)^{-1}\left(\frac{2 m}{\hbar^{2}}\right)^{2} \iint \exp \left\{i\left(\mathbf{k}_{1} \cdot \mathbf{r}_{2}^{\prime}-\mathbf{k}_{n} \cdot \mathbf{r}_{2}\right)\right\} \\
& \times \frac{\exp \left(i k\left|\mathbf{r}_{2}-\mathbf{r}_{2}^{\prime}\right|\right)}{4 \pi\left|\mathbf{r}_{2}-\mathbf{r}_{2}^{\prime}\right|} U_{n 1}\left(\mathbf{r}_{2}, \mathbf{r}_{2}{ }^{\prime}\right) d \mathbf{r}_{2} d \mathbf{r}_{2}{ }^{\prime}
\end{aligned}
$$

where

$$
\begin{aligned}
U_{n 1}\left(\mathrm{r}_{2}, \mathrm{r}_{2}^{\prime}\right)=\int \psi_{n} *\left(\mathrm{r}_{1}\right)\left(\frac{e^{2}}{\left|\mathrm{r}_{1}-\mathrm{r}_{2}\right|}-\frac{e^{2}}{r_{2}}\right) \\
\times\left(\frac{e^{2}}{\left|\mathrm{r}_{1}-\mathrm{r}_{2}{ }^{\prime}\right|}-\frac{e^{2}}{r_{2}^{\prime}}\right) \psi_{1}\left(\mathrm{r}_{1}\right) d \mathrm{r}_{1} .
\end{aligned}
$$

Expression (53) for $f_{n}{ }^{(2)}$ has been used by Rothenstein (1954) to investigate the $1 s-2 p$ excitation of hydrogen atoms by electron impact. Unfortunately this simplification of the second Born approximation does not produce reliable scattering amplitudes.

Defining the quantities $\alpha=\operatorname{Re}\left(f_{n}{ }^{(2)} / f_{n}{ }^{(1)}\right)$ and $\beta=$ $\operatorname{Im}\left(f_{n}{ }^{(2)} / f_{n}{ }^{(1)}\right)$ we see that the differential cross section given by the second Born approximation is

$$
I_{n}(\theta, \phi)=\left(k_{n} / k_{1}\right)\left|f_{n}^{(1)}(\theta, \phi)\right|^{2}\left\{(1+\alpha)^{2}+\beta^{2}\right\} .
$$

We note however that this neglects a term of the fourth order in the interaction energy $V$ contributed by the real part of the third Born approximation correction term $f_{n}{ }^{(3)}$ while including other terms of the same order and thus may well give rise to considerable error. Consequently it should be more satisfactory to neglect all fourth-order terms and use the formula

$$
I_{n}(\theta, \phi)=\left(k_{n} / k_{1}\right)\left|f_{n}^{(1)}(\theta, \phi)\right|^{2}(1+2 \alpha),
$$




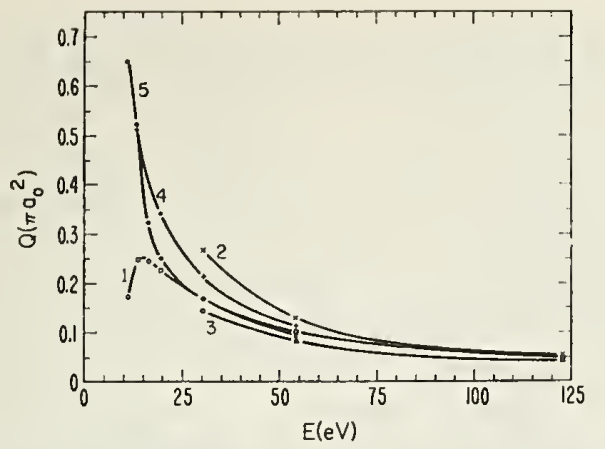

Fig. 12. Total cross sections for the $1 s \rightarrow 2 s$ excitation of atomic hydrogen. Curve 1: first Born approximation (Omidvar, 1964). Curve 2: third order $1 s-2 s$ coupling approximation (Kingston, Moiseiwitsch, and Skinner, 1960). Curve 3: third order $1 s-2 s-2 p$ coupling approximation (Kingston, Moiseiwitsch, and Skinner, 1960). Curve 4: $1 s-2 s$ close coupling approximation neglecting exchange (Smith, Miller, and Mumford, 1960). Curve 5: $1 s-2 s-2 p$ close coupling approximation neglecting exchange (Omidvar, 1964).

which is correct to the third order in the interaction energy.

The third-order approximation formula (56) has been used to investigate the $1 s \rightarrow 2 s$ excitation (Kingston, Moiseiwitsch, and Skinner, 1960) and the $1 s \rightarrow 2 p$ excitation (Moiseiwitsch and Perrin, 1965) employing a simplification of the full expression (51) obtained by truncating the summation at the states $2 s, 2 p 0,2 p \pm 1$ with principal quantum number 2 and neglecting all states with higher principal quantum numbers. The results of these calculations are given in Figs. 12 and 13. We see that for the $1 s \rightarrow 2 s$ excitation the over-all effect of allowing for coupling to the initial and final states, often referred to as distortion, is to increase the excitation cross section above that given by the first Born approximation, while allowance for coupling to the $2 p 0$ and $2 p \pm 1$ states, which should be a very important contributor to the effect known as polarization arising from coupling to all states other than the initial and final states, reduces the excitation cross section below the first Born approximation curve. These conclusions regarding the effect of distortion and polarization are in general accordance with the results of close coupling calculations with exchange neglected (Smith, Miller, and Mumford, 1960; Omidvar, 1964b) which will be discussed in Sec. 2.15.

With regard to the $1 s \rightarrow 2 p$ excitation we see from Fig. 13 that the over-all effect of the coupling to the $1 s, 2 s$, $2 p 0$, and $2 p \pm 1$ states is to increase the excitation cross section above the first Born approximation curve at the lower electron impact energies but to reduce it below the first Born approximation curve at the higher energies, again in general accordance with the behavior revealed by close coupling calculations with exchange neglected (Omidvar, 1964b).

Allowance for coupling to higher levels has been made recently by Holt and Moiseiwitsch (1968) who have evaluated exactly the contribution to the second Born approximation scattering amplitude from all intermediate levels up to $p=5$ and have estimated the con- tribution from levels with $p \geq 6$ by setting $k_{p}=k_{6}$ and using the closure relation (52). They find that the effect of the couplings to the levels with $p \geq 3$ is to bring the excitation cross sections closer to the first Born approximation at high energies and that the $1 s \rightarrow 2 p$ excitation cross section given by the second Born approximation now lies slightly below the first Born approximation curve at all energies.

\subsection{Exchange Scattering}

So far we have not taken electron exchange into account and because of its very great importance, particularly at low electron impact energies, we now turn our attention toward methods by which its inclusion may be achieved. We express the total space wave function describing the system in the symmetrized forms

$$
\Psi^{ \pm}\left(r_{1}, r_{2}\right)=\Psi\left(r_{1}, r_{2}\right) \pm \Psi\left(r_{2}, r_{1}\right),
$$

where $\Psi\left(r_{1}, r_{2}\right)$ is given by the expansion (3) and the positively and negatively symmetrized functions $\Psi^{+}$ and $\Psi^{-}$are associated with singlet and triplet scattering, respectively. Then the entire wave function of the system composed of a spin as well as a space part is totally antisymmetric with respect to interchange of all the coordinates, both space and spin, of electrons 1 and 2 thus satisfying the Pauli exclusion principle.

Substituting (57) into the Schrödinger equation (1), multiplying across by $\psi_{n} *\left(\mathbf{r}_{1}\right)$, and integrating with respect to $r_{1}$, yields

$$
\left(\nabla_{2}^{2}+k_{n}^{2}\right) F_{n}^{ \pm}\left(\mathbf{r}_{2}\right)=\left(2 m / \hbar^{2}\right) \underset{p}{S} R_{n p} \pm\left(\mathbf{r}_{2}\right),
$$

where

$$
\begin{array}{r}
R_{n p}^{ \pm}\left(\mathrm{r}_{2}\right)=F_{p} \pm\left(\mathrm{r}_{2}\right) \int \psi_{n} *\left(\mathrm{r}_{1}\right) V_{2}\left(\mathrm{r}_{1}, \mathrm{r}_{2}\right) \psi_{p}\left(\mathrm{r}_{1}\right) d \mathrm{r}_{1} \\
\pm \psi_{p}\left(\mathrm{r}_{2}\right) \int \psi_{n} *\left(\mathrm{r}_{1}\right)\left\{V_{1}\left(\mathrm{r}_{1}, \mathrm{r}_{2}\right)\right. \\
\left.-\left(\hbar^{2} / 2 m\right)\left(\nabla_{1}^{2}+k_{p}^{2}\right)\right\} F_{p} \pm\left(\mathrm{r}_{1}\right) d \mathrm{r}_{1},
\end{array}
$$

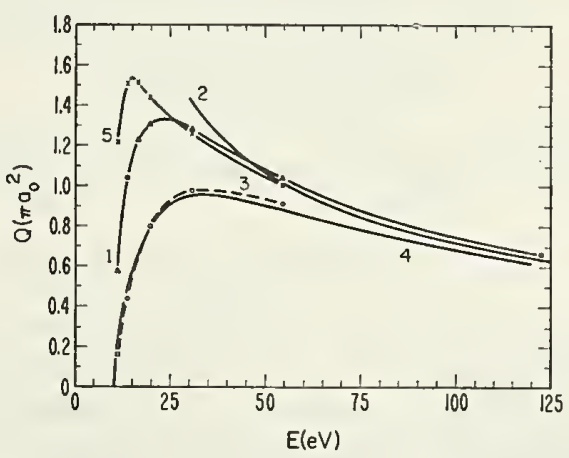

FIG. 13. Total cross sections for the $1 s \rightarrow 2 p$ excitation of atomic hydrogen. Curve 1: first Born approximation (Omidvar, 1964). Curve 2: third-order $1 s-2 s-2 p$ coupling approximation (Moiseiwitsch and Perrin, 1965). Curve 3: unitarized Born approximation (Burke and Seaton, 1961). Curve 4: impact parameter method (Seaton, 1962). Curve 5: $1 s-2 s-2 p$ close coupling approximation neglecting exchange (Omidvar, 1964). 
with

$$
V_{1}\left(\mathbf{r}_{1}, \mathbf{r}_{2}\right)=\left(e^{2} / r_{12}\right)-\left(e^{2} / r_{1}\right)
$$

being the interaction energy between electron 1 and a hydrogen atom composed of electron 2 and the proton, and

$$
V_{2}\left(\mathbf{r}_{1}, \mathbf{r}_{2}\right)=\left(e^{2} / r_{12}\right)-\left(e^{2} / r_{2}\right)
$$

being the interaction energy between electron 2 and a hydrogen atom composed of electron 1 and the proton.

Now using the Green's function (10) for a free particle we obtain the following expression for the scattering amplitude:

$f_{n} \pm(\theta, \phi)=-(4 \pi)^{-1} \frac{2 m}{\hbar^{2}} \mathbf{S}_{p} \int \exp \left(-i \mathbf{k}_{n} \cdot \mathbf{r}_{2}\right) R_{n p} \pm\left(\mathbf{r}_{2}\right) d \mathbf{r}_{2}$.

The $1 \rightarrow n$ excitation cross sections $Q_{n}{ }^{+}$and $Q_{n}{ }^{-}$corresponding to singlet and triplet scattering are given by

$$
Q_{n}^{ \pm}=\frac{k_{n}}{k_{1}} \iint\left|f_{n}^{ \pm}(\theta, \phi)\right|^{2} \sin \theta d \theta d \phi
$$

and since they have spin statistical weighting factors $\frac{1}{4}$ and $\frac{3}{4}$, respectively, the total $1 \rightarrow n$ excitation cross section takes the form

$$
Q_{n}=\frac{1}{4} Q_{n}{ }^{+}+\frac{3}{4} Q_{n}^{-} \text {. }
$$

\subsection{Born-Oppenheimer Approximation}

We now attempt to make allowance for exchange to the same order of accuracy as that used to derive the first Born approximation. Again considering the case of electrons incident upon a target hydrogen atom in its ground state, we set

$$
\begin{aligned}
& F_{1}^{ \pm}(\mathbf{r})=\exp \left(i \mathbf{k}_{1} \cdot \mathbf{r}\right) \\
& F_{p}^{ \pm}(\mathbf{r})=0 \quad(p \neq 1)
\end{aligned}
$$

in formula (59) for $R_{n p} \pm$ and substitute the resulting expression into (62). Then we obtain the so called Born-Oppenheimer approximation to the scattering amplitude which is given by

$$
\begin{aligned}
f_{n} \pm(\theta, \phi)= & -(4 \pi)^{-1} \frac{2 m}{\hbar^{2}} \iint \exp \left\{i\left(\mathbf{k}_{1}-\mathbf{k}_{n}\right) \cdot \mathbf{r}_{2}\right\} \psi_{n} *\left(\mathbf{r}_{1}\right) \\
& \times V_{2}\left(\mathbf{r}_{1}, \mathbf{r}_{2}\right) \psi_{1}\left(\mathbf{r}_{1}\right) d \mathbf{r}_{1} d \mathbf{r}_{2} \\
\mp(4 \pi)^{-1} & \frac{2 m}{\hbar^{2}} \iint \exp \left\{i\left(\mathbf{k}_{1} \cdot \mathbf{r}_{1}-\mathbf{k}_{n} \cdot \mathbf{r}_{2}\right)\right\} \\
& \times \psi_{n}{ }^{*}\left(\mathbf{r}_{1}\right) V_{1}\left(\mathbf{r}_{1}, \mathbf{r}_{2}\right) \psi_{1}\left(\mathbf{r}_{2}\right) d \mathbf{r}_{1} d \mathbf{r}_{2} .
\end{aligned}
$$

The first term on the right-hand side of this formula for the scattering amplitude is just the first Born approximation while the second term is the additional contribution arising from exchange according to the BornOppenheimer approximation.

Closed analytical expressions for the scattering amplitude corresponding to the $1 s \rightarrow 2 s$ and $1 s \rightarrow 2 p 0$ excitations have been obtained by Corinaldesi and Trainor (1952) using the Feynman parametrization technique, the axis of quantization of the atomic wave functions being chosen along the direction of incidence of the free electron. They found the exchange contribution to the scattering amplitude for the $1 s \rightarrow 2 p \pm 1$ excitation to be zero but this is not so, the correct expression being given by Bell (1965) for this case. Calculations of the Born-Oppenheimer cross sections for the $1 s \rightarrow 2 s, 1 s \rightarrow$ $2 p 0$, and $1 s \rightarrow 2 p \pm 1$ excitations have been performed by Wu (1960) but contain numerical errors, by Bell and Moiseiwitsch (1963) who took the exchange amplitude for the $1 s \rightarrow 2 p \pm 1$ excitation to vanish, and by Bell (1965) who used the correct expression for the $1 s \rightarrow$ $2 p \pm 1$ exchange amplitude. The results of these calculations are presented in Tables XII and XIII. We see that for the $1 s \rightarrow 2 s$ excitation, the Born-Oppenheimer approximation yields a cross section which is much larger close to the threshold energy than that given by the first Born approximation. This cannot be correct: thus for electrons with $13.6-\mathrm{eV}$ energy corresponding to $k_{1}=1$ in units of $a_{0}^{-1}$, the zero-order partial cross section given by the Born-Oppenheimer approximation has magnitude $1.59 \pi a_{0}^{2}$ whereas the maximum possible cross section for the $\ell$ th-order partial wave imposed by the conservation theorem of Bohr, Peierls, and Placzek (Mott and Massey, 1965a) is $(2 \ell+1) \pi a_{0}^{2} / k_{1}^{2}$ so that the Born-Oppenheimer approximation violates the theoretical maximum for $\ell=0$ at $k_{1}=1$. As we see later, this gross overestimation of the excitation cross section produced by the Born-Oppenheimer approximation is a typical occurrence for $S \rightarrow S$ transitions. On the other hand, by referring to Table XIII, we see that the Born-Oppenheimer approximation predicts a rather smaller exchange effect for the $1 s \rightarrow 2 p$ excitations.

\subsection{First-Order Exchange Approximation}

The Born-Oppenheimer approximation (66) suffers from the undesirable feature that the addition of a constant to the interaction potential $V_{2}$ produces a nonvanishing alteration in the exchange scattering amplitude owing to the lack of orthogonality between the approximate wave function $\Psi_{1}\left(\mathbf{r}_{1}\right) \exp \left(i \mathbf{k}_{1} \cdot \mathbf{r}_{2}\right)$ describing the initial state of the system and the approximate wave function $\Psi_{n}\left(\mathbf{r}_{2}\right) \exp \left(i \mathbf{k}_{n} \cdot \mathbf{r}_{1}\right)$ describing the state of the system after an exchange event involving the excitation of the atom has occurred. This arbitrariness of the Born-Oppenheimer approximation can be seen to arise because $\left(\nabla^{2}+k_{1}^{2}\right) F_{1^{ \pm}}$vanishes for $F_{1}^{ \pm}=$ $\exp \left(i \mathbf{k}_{1} \cdot \mathbf{r}\right)$ whereas it is clear from (58) that this term is actually of the first order in the interaction potential. Thus neglecting exchange and the coupling of the initial state to all other states of the system we see that equation (58) gives us

$$
\begin{aligned}
& \left(\nabla_{2}{ }^{2}+k_{1}{ }^{2}\right) F_{1}^{ \pm}\left(r_{2}\right) \\
& \quad=\left(2 m / \hbar^{2}\right) F_{1}^{ \pm}\left(r_{2}\right) \int\left|\Psi_{1}\left(r_{1}\right)\right|^{2} V_{2}\left(r_{1}, r_{2}\right) d r_{1} .
\end{aligned}
$$

[Note that the argument can be carried through without these simplifying assumptions (Bell and Moiseiwitsch, 1963).] 
Replacing $\left(\nabla^{2}+k_{1}^{2}\right) F_{1}^{ \pm}$by the right-hand side of (67) and then making approximation (65), we now get in place of the Born-Oppenheimer approximation:

$f_{n}^{ \pm}(\theta, \phi)=-(4 \pi)^{-1} \frac{2 m}{\hbar^{2}} \iint \exp \left\{i\left(\mathbf{k}_{1}-\mathbf{k}_{n}\right) \cdot \mathbf{r}_{2}\right\} \psi_{n} *\left(\mathbf{r}_{1}\right) V_{2}\left(\mathbf{r}_{\mathbf{1}}, \mathbf{r}_{2}\right) \psi_{1}\left(\mathbf{r}_{1}\right) d \mathbf{r}_{1} d \mathbf{r}_{2} \mp(4 \pi)^{-1} \frac{2 m}{\hbar^{2}} \iint \exp \left\{i\left(\mathbf{k}_{1} \cdot \mathbf{r}_{1}-\mathbf{k}_{n} \cdot \mathbf{r}_{2}\right)\right\}$

$$
\times \psi_{n}^{*}\left(\mathbf{r}_{1}\right) \psi_{1}\left(\mathbf{r}_{2}\right)\left\{V_{1}\left(\mathbf{r}_{1}, \mathbf{r}_{2}\right)-\int\left|\psi_{1}\left(\mathbf{r}_{1}^{\prime}\right)\right|^{2} V_{1}\left(\mathbf{r}_{1}^{\prime}, \mathbf{r}_{2}\right) d \mathbf{r}_{1}^{\prime}\right\} d \mathbf{r}_{1} d \mathbf{r}_{2}
$$

which remains unaltered if a constant is added to the interaction potential $V_{1}$. This formula was first derived by Feenberg (1932). It differs from the Born-Oppenheimer approximation by the replacement of the electron-proton interaction $e^{2} / r_{1}$ in $V_{1}$ by

$$
\int\left|\Psi_{1}\left(\mathbf{r}_{1}^{\prime}\right)\right|^{2} \frac{e^{2}}{r_{12}} d r_{1}^{\prime}
$$

which is the average of the electron-electron interaction $e^{2} / r_{12}$ over the initial state of the hydrogen atom. The Feenberg approximation can be derived alternatively by using a variational principle for the scattering amplitude together with distorted wave trial functions, and subsequently retaining only those terms of the scattering amplitude which are of the first order in the interaction energy. It seems therefore to be more securely founded than the Born-Oppenheimer approximation which follows from the use of a variational principle in conjunction with plane wave trial functions. However the attention of the reader is directed to the treatise by Mott and Massey (1965b) where a different attitude is taken.

If instead of using equation (67) we employ the true equation satisfied by $F_{1}^{ \pm}$, the resulting scattering amplitude contains certain additional small terms which have been excluded from formula (68). With these terms retained the approximation to the scattering amplitude is known as the first-order exchange approximation. It has been employed to calculate the cross sections for the $1 s \rightarrow 2 s$ and $1 s \rightarrow 2 p$ excitations by Bell and Moiseiwitsch (1963) and by Bell (1965). The results of these calculations are displayed in Tables XII and XIII where they are compared with those obtained with the Born-Oppenheimer approximation. It is seen that for the $1 s \rightarrow 2 s$ excitation the use of the first-order exchange approximation produces a considerable reduction in the cross section below that given by the Born-Oppenheimer approximation at low electron impact energies. Moreover the zero-order partial cross section given by the first-order exchange approximation has magnitude $0.39 \pi a_{0}^{2}$ so that, unlike the Born-Oppenheimer approximation, the upper bound imposed by the conservation theorem is not violated. Thus the first-order exchange approximation is the better approximation for the case of the $1 s \rightarrow 2 s$ excitation of atomic hydrogen. We shall see later that this appears to be generally so for $S \rightarrow S$ transitions.
For the $1 s \rightarrow 2 p 0$ and $2 p \pm 1$ excitations the difference between the cross sections given by the Born-Oppenheimer and the first-order exchange approximations is negligible except at energies close to the threshold.

Inspection of Tables XII and XIII shows that according to the first-order exchange and the BornOppenheimer approximations, the effect of exchange is to reduce the $1 s \rightarrow 2 s$ and $1 s \rightarrow 2 p$ excitation cross sections for electron impact energies away from the threshold.

\subsection{Ochkur Approximation}

Another approach to the problem of overcoming the deficiencies of the Born-Oppenheimer approximation is due to Ochkur (1963). He expands the exchange scattering amplitude

$$
\begin{array}{r}
g_{n}(\theta, \phi)=-(4 \pi)^{-1} \frac{2 m e^{2}}{\hbar^{2}} \iint \exp \left\{i\left(\mathbf{k}_{1} \cdot \mathbf{r}_{1}-\mathbf{k}_{n} \cdot \mathbf{r}_{2}\right)\right\} \\
\times \psi_{n}{ }^{*}\left(\mathbf{r}_{1}\right)\left(r_{12}{ }^{-1}-r_{1}^{-1}\right) \psi_{1}\left(\mathbf{r}_{2}\right) d \mathbf{r}_{1} d \mathbf{r}_{2}
\end{array}
$$

given by the Born-Oppenheimer approximation in powers of $k_{1}^{-1}$ and retains only the leading term which behaves as $k_{1}^{-2}$. The electron-proton interaction $c^{2} / r_{1}$ results in a contribution which decays more rapidly than $k_{1}^{-2}$ and so is neglected.

To obtain the leading term arising from the electronelectron interaction $e^{2} / r_{12}$ we note that

$$
\left(4 \pi r_{12}\right)^{-1}=\frac{1}{(2 \pi)^{3}} \int \exp \left\{i \mathrm{q} \cdot\left(\mathrm{r}_{1}-\mathrm{r}_{2}\right)\right\} \frac{d \mathrm{q}}{q^{2}} .
$$

Since the main contribution to $g_{n}$ comes from $q \simeq k_{1}$, we have to the lowest order in $k_{1}^{-1}$

$$
\left(r_{12}\right)^{-1} \cong\left(4 \pi / k_{1}^{2}\right) \delta\left(\mathbf{r}_{1}-\mathbf{r}_{2}\right)
$$

remembering that the Dirac $\delta$ function can be expressed in the form

$$
\delta(\mathbf{r})=\frac{1}{(2 \pi)^{3}} \int \exp (i \mathbf{q} \cdot \mathbf{r}) d \mathbf{q} .
$$

Hence

$g_{n}(\theta, \phi) \cong-\frac{2 m e^{2}}{\hbar^{2} k_{1}{ }^{2}} \int \exp \left(i \mathbf{K} \cdot \mathbf{r}_{2}\right) \psi_{n} *\left(\mathbf{r}_{2}\right) \psi_{1}\left(\mathbf{r}_{2}\right) d \mathbf{r}_{2}$,

where $\mathbf{K}=\mathbf{k}_{1}-\mathbf{k}_{n}$, giving for the entire scattering amplitude according to the Uchkur approximation:

$f_{n} \pm(\theta, \phi)=-\left(2 / a_{0}\right)\left[\left(1 / K^{2}\right) \pm\left(1 / k_{1}^{2}\right)\right] g(1-n)$. 
The calculations using this approximation have been confined so far to helium excitation and are discussed in Sec. 3.2. [Note added in proof. Recently Inokuti (1967) has used the Ochkur approximation to calculate the $1 s \rightarrow 2 s$ and $1 s \rightarrow 2 p$ excitation cross sections for atomic hydrogen. As we see there, the Ochkur approximation appears to be not only a very useful one but also rather simple to apply since it is no more difficult to evaluate than the first Born approximation.]

A modification of the Ochkur approximation based on a variational principle has been derived by Rudge (1965). He has applied his formula to the $1 s \rightarrow 2 s$ excitation of atomic hydrogen, obtaining a cross-section curve which is substantially smaller than that given by the first Born approximation and also by the $1 s-2 s$ close coupling calculation with exchange included (Omidvar, 1964b).

\subsection{Distorted Waves Approximation}

We have already examined the use of the second Born approximation in making partial allowance for the effect of distortion. Full allowance for the distortion of the incident and scattered electron waves can be achieved in the following manner. We make a two-state approximation involving just the initial state, which we suppose to be the ground state 1 of the hydrogen atom, and the final state $n$, and neglect coupling to all other states. Then the infinite set of coupled equations (58) reduce to the pair of equations

$$
\begin{aligned}
& {\left[\nabla_{2}^{2}+k_{1}^{2}-\frac{2 m}{\hbar^{2}} V_{11}\left(\mathbf{r}_{2}\right)\right] F_{1}^{ \pm}\left(\mathbf{r}_{2}\right) \mp \frac{2 m}{\hbar^{2}} \int K_{11}\left(\mathbf{r}_{1}, \mathbf{r}_{2}\right) F_{1} \pm\left(\mathbf{r}_{1}\right) d \mathbf{r}_{1}=\frac{2 m}{\hbar^{2}}\left\{V_{1 n}\left(\mathbf{r}_{2}\right) F_{n} \pm\left(\mathbf{r}_{2}\right) \pm \int K_{1 n}\left(\mathbf{r}_{1}, \mathbf{r}_{2}\right) F_{n} \pm\left(\mathbf{r}_{1}\right) d \mathbf{r}_{1}\right\}} \\
& {\left[\nabla_{2}^{2}+k_{n}{ }^{2}-\frac{2 m}{\hbar^{2}} V_{n n}\left(\mathbf{r}_{2}\right)\right] F_{n} \pm\left(\mathbf{r}_{2}\right) \mp \frac{2 m}{\hbar^{2}} \int K_{n n}\left(\mathbf{r}_{1}, \mathbf{r}_{2}\right) F_{n} \pm\left(\mathbf{r}_{1}\right) d \mathbf{r}_{1}=\frac{2 m}{\hbar^{2}}\left\{V_{n 1}\left(\mathbf{r}_{2}\right) F_{1}^{ \pm}\left(\mathbf{r}_{2}\right) \pm \int K_{n 1}\left(\mathbf{r}_{1}, \mathbf{r}_{2}\right) F_{1} \pm\left(\mathbf{r}_{1}\right) d \mathbf{r}_{1}\right\},}
\end{aligned}
$$

where the matrix elements $V_{n 1}\left(r_{2}\right)$ of the interaction energy $V\left(r_{1}, r_{2}\right)$ are given by the formula (17) and the kernels $K_{n 1}\left(\mathrm{I}_{1}, \mathrm{r}_{2}\right)$ have the form

$$
K_{n 1}\left(\mathbf{r}_{1}, \mathbf{r}_{2}\right)=\psi_{1}\left(\mathbf{r}_{2}\right) \psi_{n}^{*}\left(\mathrm{r}_{1}\right)\left\{\left(e^{2} / r_{12}\right)+E_{1}+E_{n}-E\right\} .
$$

If we know the solution of the reduced equation

$$
\begin{aligned}
{\left[\nabla_{2}^{2}+k_{n}^{2}-\frac{2 m}{\hbar^{2}}\right.} & \left.V_{n n}\left(\mathbf{r}_{2}\right)\right] \widetilde{\digamma}_{n}^{ \pm}\left(\mathbf{r}_{2}\right) \\
& \mp \frac{2 m}{\hbar^{2}} \int K_{n n}\left(\mathbf{r}_{1}, \mathbf{r}_{2}\right) \widetilde{F}_{n}^{ \pm}\left(\mathbf{r}_{1}\right) d \mathbf{r}_{1}=0
\end{aligned}
$$

obtained by setting the right-hand side of (76) equal to zero and choosing the asymptotic behavior for large $r$ to be

$$
\tilde{\digamma}_{n}{ }^{ \pm}(\mathbf{r}) \sim \exp \left(-i \mathbf{k}_{n} \cdot \mathbf{r}\right)+r^{-1} \exp \left(i k_{n} r\right) \tilde{\mathscr{S}}_{n}{ }^{ \pm}(\theta, \phi)
$$

we may express the scattering amplitude $f_{n}(\theta, \phi)$ in the form

$$
\begin{aligned}
& f_{n}^{ \pm}(\theta, \phi)=-\frac{1}{4 \pi} \frac{2 m}{\hbar^{2}} \int \widetilde{F}_{n}\left(\mathbf{r}_{2}\right) \\
& \times\left\{V_{n 1}\left(\mathbf{r}_{2}\right) F_{1} \pm\left(\mathbf{r}_{2}\right) \pm \int K_{n 1}\left(\mathrm{r}_{1}, \mathrm{r}_{2}\right) F_{1} \pm\left(\mathrm{r}_{1}\right) d \mathrm{r}_{1}\right\} d \mathrm{r}_{2} .
\end{aligned}
$$

We cannot obtain $F_{1}^{ \pm}$without solving the pair of coupled equations (75) and (76). Since this requires considerable computational effort, we introduce here the so called distorted waves approximation which is based upon the assumption that the back coupling of the final state $n$ to the initial state 1 is small so that $V_{1 n}$ and $K_{1 n}$ can be set equal to zero on the right-hand side of Eq. (75) which now takes the reduced form

$$
\begin{aligned}
{\left[\nabla_{2}^{2}+k_{1}^{2}-\frac{2 m}{\hbar^{2}}\right.} & \left.V_{11}\left(\mathbf{r}_{2}\right)\right] \mathcal{F}_{1} \pm\left(\mathbf{r}_{2}\right) \\
& \mp \frac{2 m}{\hbar^{2}} \int K_{11}\left(\mathbf{r}_{1}, \mathbf{r}_{2}\right) \digamma_{1}^{ \pm}\left(\mathbf{r}_{1}\right) \mathrm{d} r_{1}=0,
\end{aligned}
$$

where $F_{1}^{ \pm}$is chosen to have the same asymptotic behavior as $F_{1}^{ \pm}$for large $r$ :

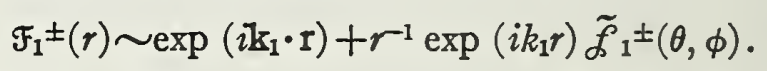

Replacing $F_{1}^{ \pm}$by $\mathcal{F}_{1}^{ \pm}$in (80) yields the distorted waves approximation to the scattering amplitude:

$$
\begin{aligned}
& f_{n}^{ \pm}(\theta, \phi)=-(4 \pi)^{-1} \frac{2 m}{\hbar^{2}} \int \tilde{F}_{n} \pm\left(\mathbf{r}_{2}\right) \\
& \times\left\{V_{n 1}\left(\mathbf{r}_{2}\right) \mathscr{F}_{1}^{ \pm}\left(\mathbf{r}_{2}\right) \pm \int K_{n 1}\left(\mathbf{r}_{1}, \mathbf{r}_{2}\right) \mathfrak{F}_{1}^{ \pm}\left(\mathbf{r}_{1}\right) d \mathbf{r}_{1}\right\} d \mathbf{r}_{2}
\end{aligned}
$$

To solve equations (78) and (81) it is necessary to make a partial waves analysis of $\digamma_{1} \pm$ and $\mathcal{F}_{r_{t}} \pm$ in which case the total excitation cross section $Q_{n}$ may be expressed in terms of the partial cross sections $Q_{n}{ }^{l \prime \prime}$ according to the formula (32). The earliest calculations employing the distorted waves method were performed by Erskine and Massey (1952) with, as well as without, the inclusion of exchange. They dealt with the $1 s \rightarrow 2 s$ excitation of atomic hydrogen and considered only the $\ell=$ 


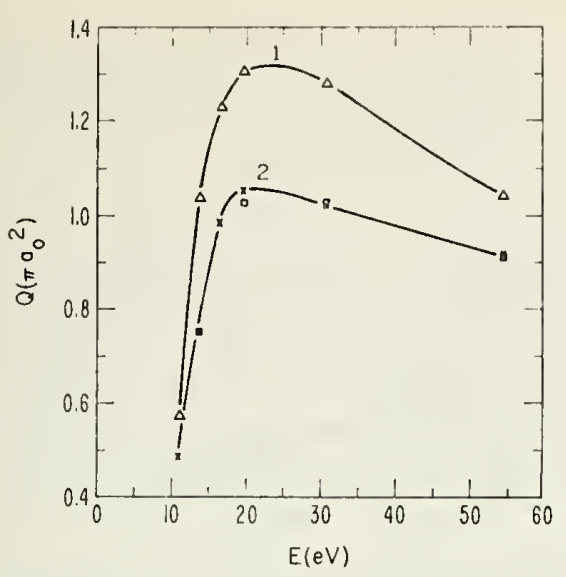

FIG. 14. Total cross sections for the $1 s \rightarrow 2 p$ excitation of atomic hydrogen. Curve 1: first Born approximation (Omidvar, 1964). Curve 2: $1 s-2 p$ close coupling approximation including exchange (Omidvar, 1964). $\square$ symbols: distorted waves approximation including exchange (Khashaba and Massey, 1958).

$\ell^{\prime}=0$ partial wave, the distorted wave functions being determined by variational methods. The exchange distorted waves calculations were subsequently repeated by Ochkur (1958) who however determined the distorted wave functions numerically using the integral equations method developed by Drukarev (1953). The zero-order partial cross sections $Q_{2 s}{ }^{0}$ calculated using the method of distorted waves are compared with the results obtained employing other forms of approximation in Table XIV. When exchange is neglected we see that the method of distorted waves produces zero-order partial cross sections which are quite close to those found using the first Born approximation, both being in satisfactory accordance with the $1 s-2 s$ close coupling calculations of Bransden and McKee (1956). However with exchange taken into account, not only are the partial cross sections $Q_{2 s}{ }^{0}{ }$ resulting from the two applications of the distorted waves method very different from those obtained using the Born-Oppenheimer approximation but also they are considerably different from each other. This is probably due to the sensitivity of the partial cross sections to the distorted wave functions which were determined in different ways by Erskine and Massey and by Ochkur. Moreover comparison with the $1 s-2 s$ close coupling calculations with exchange included carried out by Marriott (1958) shows that the distorted waves approximation fails to produce accurate zero-order partial cross sections and so we infer that the back coupling arising from exchange is not sufficiently weak to be neglected here.

Calculations uave also been carried out by Khashaba and Massey (1958) on the $1 s \rightarrow 2 p$ excitation of atomic hydrogen treating the $\ell=0$ and the $\ell^{\prime}=0$ and 1 partial waves by the distorted waves method and the higher order partial waves by the Born-Oppenheimer approximation, variational methods being employed to determine the distorted wave functions. They found that the $Q_{2 p}^{1,0}$ partial cross section was greatly reduced below that given by the Born-Oppenheimer approximation as a consequence of making allowance for distortion. Ochkur has repeated the calculation of the 1, 0 partial cross section using an $\ell^{\prime}=0$ distorted wave function for the scattered electron, determined with the aid of the integral equation method due to Drukarev, and using an undistorted $\ell=1$ wave function for the incident electron. The results of Ochkur's calculations are not in satisfactory accordance with those obtained by Khashaba and Massey.

In Fig. 14 we make a comparison between the $1 s \rightarrow 2 p$ total excitation cross section calculated by Khashaba and Massey and the $1 s-2 p$ close coupling approximation cross section. The agreement between the two approximations is quite good, both of them leading to total excitation cross sections lying somewhat below the first Born approximation curve.

Vainshtein (1961) has examined the $1 s \rightarrow 2 s, 1 s \rightarrow 2 p$, $1 s \rightarrow 3 s, 1 s \rightarrow 3 p, 1 s \rightarrow 3 d, 1 s \rightarrow 4 s, 1 s \rightarrow 4 p$, and $2 s \rightarrow 3 p$, $2 p \rightarrow 3 s$ excitations of hydrogen atoms treating the partial cross sections with $\ell, \ell^{\prime} \leq 6$ by the distorted waves method, but neglecting exchange, and calculating the higher order partial cross sections using the Born approximation. The resulting total cross sections are displayed in Table XV. A noteworthy feature of these values is that the distorted waves method without exchange gives larger cross sections than the Born approximation at low energies. This occurs because allowance for distortion involves the introduction of an attractive potential so that the wave functions are pulled in and the cross sections increased as a consequence.

We also show the partial cross sections obtained by Vainshtein for the $1 s \rightarrow 2 s$ and $1 s \rightarrow 2 p$ excitations using the distorted waves method in Figs. 15 and 16. It can be readily seen that for the $1 s \rightarrow 2 p$ excitation the 1,0

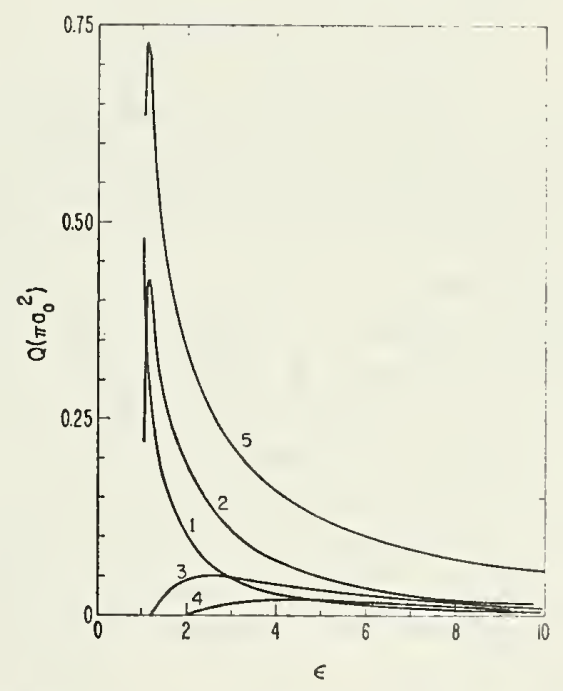

FIg. 15. Partial cross sections for the $1 s \rightarrow 2 s$ excitation of atomic hydrogen calculated using the distorted waves method neglecting exchange (Vainshtein, 1961). [ $\epsilon$ is electron energy in threshold units defined by Eq. (38).] Curve 1: $Q_{2 s^{\circ}}$, Curve 2: $Q_{2}{ }^{1}$, Curve 3: $Q_{2 s}{ }^{2}$, Curve 4: $Q_{2 s^{3}}$,

Curve 5: $\sum_{\ell=0}^{6} Q_{2} \ell$. 
and the 0,1 partial cross sections make a negligible contribution to the total excitation cross section, the 1,0 curve being too small to be distinguished on the figure. This is quite different from the result obtained with the first Born approximation which gives the 1,0 partial cross section as the primary contributor to the total cross section. At low electron energies the distorted waves method gives the 2, 1 partial cross section as the most important. Also, as one would expect, it is found that the $\ell, \ell-1$ partial cross sections are much larger than the $\ell, \ell+1$ cross sections.

\subsection{Modified Bethe Approximation}

In all the previous sections we have assumed that the coupling between the initial and final states is weak. In certain cases however this coupling is obviously very strong. An example of very strong coupling is the $2 s \rightarrow 2 p$ excitation of atomic hydrogen. It is found that when the Born approximation is applied to it, the partial cross sections $Q^{\ell}$, associated with incident electrons having angular momentum $\hbar[\ell(\ell+1)]^{1 / 2}$, exceed the theoretical maximum $(2 \ell+1) \pi a_{0}^{2} / k_{2}^{2}$ imposed by the conservation theorem (Mott and Massey, 1965a) when $\ell$ is small. A method of dealing with this difficulty has been developed by Seaton (1955a). Let $\ell_{0}$ be the value of $l$ for which

$$
\begin{aligned}
& Q_{\text {Bethe }}{ }^{\ell}<\frac{1}{2}(2 \ell+1) \pi a_{0}{ }^{2} / k_{2}{ }^{2} \quad\left(\ell>\ell_{0}\right) \\
& \geq \frac{1}{2}(2 \ell+1) \pi a_{0}{ }^{2} / k_{2}{ }^{2} \quad\left(\ell \leq \ell_{0}\right),
\end{aligned}
$$

where $Q_{B \text { ethe }}{ }^{\ell}$ is the partial cross section given by the Bethe approximation. The modification of the Bethe approximation suggested by Seaton sets

$$
\begin{aligned}
Q^{\ell} & =Q_{\text {Bethe }^{\ell}} \quad\left(\ell>\ell_{0}\right) \\
& =\frac{1}{2}(2 \ell+1) \pi a_{0}{ }^{2} / k_{2}{ }^{2}\left(\ell \leq \ell_{0}\right)
\end{aligned}
$$

and is based on the plausible assumption that the partial cross sections for $\ell \leq \ell_{0}$ can be replaced by their average value $\left[\frac{1}{2}(2 \ell+1) \pi a_{0}{ }^{2} / k_{2}{ }^{2}\right]$ without undue error. Using this approximation Seaton has calculated the rate coefficients for the $2 s \rightarrow 2 p_{1 / 2}$ and $2 s \rightarrow 2 p_{3 / 2}$ transitions in atomic hydrogen at temperatures of $10000^{\circ} \mathrm{K}$ and $20000^{\circ} \mathrm{K}$ assuming a Maxwellian velocity distribution of electrons and taking the energy differences between the upper and lower states to be 0.0354 and $0.327 \mathrm{~cm}^{-1}$, respectively. The rate coefficients are given in Table XVI where they are compared with the values obtained with the Born approximation, the usual Bethe approximation and a semi-classical approximation (Purcell, 1952).

Allowance for strong coupling by means of the modified Bethe approximation decreases the rate coefficients only slightly for the case under consideration because most of the contribution to the cross sections comes from distant encounters so that large values of $l$ dominate and the first Born approximation is fairly accurate. The effect of very strong coupling is most important

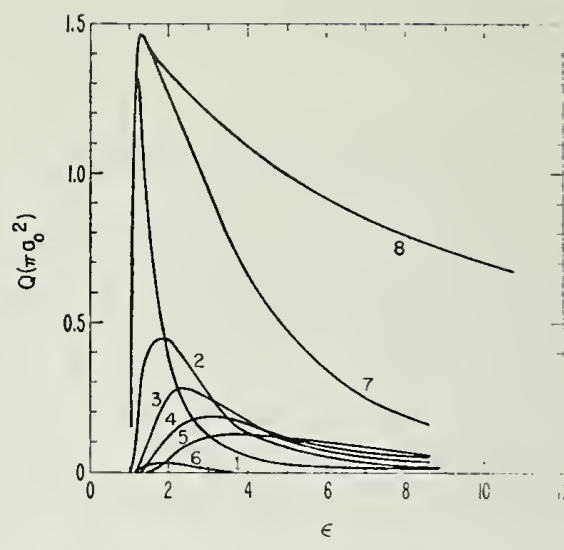

FIG. 16. Partial cross sections for the $1 s \rightarrow 2 p$ excitation of atomic hydrogen calculated using the distorted waves method neglecting exchange (Vainshtein, 1961). [ $\epsilon$ is electron energy in threshold units defined by Eq. (38).] Curve 1: $Q_{2 p^{2,1}}$, Curve 2: $Q_{2 p}{ }^{3,2}$, Curve 3: $Q_{2 p^{4,3}}$, Curve 4: $Q_{2 p}{ }^{5,4}$, Curve 5: $Q_{2 p}{ }^{6,5}$, Curve 6: $Q^{0,1}$,

Curve 7: $\sum_{\ell=0}^{6} Q_{2 p}^{\ell}, \quad$ Curve 8: $Q_{2 p}$.

for $n \rightarrow n+1$ transitions of atomic hydrogen discussed in the succeeding section and, for example, in the case of the $3 s \rightarrow 3 p$ excitation of sodium examined in Sec. 6 .

\subsection{Impact Parameter Method}

In this section we shall be concerned with the use of the semiclassical method introduced by Seaton (1962). Known as the impact parameter method, it is based upon the assumption that the path of the incident electron can be taken to be a straight line whose perpendicular distance from the atomic nucleus is the impact parameter $\rho$. If $P_{n \mathbf{1}}(\rho)$ denotes the probability that an incident electron having impact parameter $\rho$ produces a transition from the ground state 1 to an excited state $n$ of the target atom, the total $1 \rightarrow n$ excitation cross section can be expressed in the form

$$
Q_{n}=2 \pi \int_{0}^{\infty} P_{n 1}(\rho) \rho d \rho .
$$

The transition probability $P_{n 1}$ is evaluated using firstorder time-dependent perturbation theory which gives

$$
P_{n 1}=\left|\hbar^{-1} \int_{-\infty}^{\infty} \exp \left\{i\left(E_{n}-E_{1}\right) t / \hbar\right\} V_{n 1}(t) d t\right|^{2},
$$

where $E_{1}$ and $E_{n}$ are the energies of the initial and final states of the atom, and $V_{n 1}(t)$ is the matrix element of the Coulomb interaction between the incident electron and the target atom associated with the initial and final states. In the case of hydrogen

$$
V_{n 1}(t)=\int \psi_{n}^{*}\left(\mathbf{r}_{1}\right) \frac{e^{2}}{\left|\mathbf{r}_{1}-\mathbf{r}_{2}(t)\right|} \psi_{1}\left(\mathbf{r}_{1}\right) d \mathbf{r}_{1}
$$

where $\psi_{1}$ and $\psi_{n}$ are the atomic wave functions corresponding to the states 1 and $n$, respectively. The electron-electron interaction $e^{2} /\left|r_{1}-r_{2}\right|$ may be expressed 
as a multipole expansion using the formula

$$
\left|\mathbf{r}_{1}-\mathrm{r}_{2}\right|^{-1}=\sum_{\lambda=0}^{\infty} \frac{r_{<}^{\lambda}}{r>\lambda+1} P_{\lambda}(\cos \theta),
$$

where $r_{<}$and $r_{>}$are the lesser and the greater of $r_{1}$ and $r_{2}, \theta$ is the angle between $\mathbf{r}_{1}$ and $\mathbf{r}_{2}$, and the $P_{\lambda}(\cos \theta)$ are the Legendre polynomials. For optically allowed transitions $1 s \rightarrow n p$, the only term of the multipole expansion which provides a nonvanishing contribution to $V_{n 1}$ is the dipole term. When $\rho$ is large we may replace $V_{n 1}$ by its asymptotic form for large separations. Since the dipole term corresponding to $\lambda=1$ takes the form $e^{2} \mathbf{r}_{1} \cdot \mathbf{r}_{2} / r_{2}^{3}$ for large $\boldsymbol{r}_{2}$, we see that

$$
V_{n 1}\left(r_{2}\right) \sim e^{2} \frac{\mathbf{r}_{2}}{r_{2}^{3}} \cdot \int \psi_{n} *\left(\mathbf{r}_{1}\right) \mathbf{r}_{1} \psi_{1}\left(\mathbf{r}_{1}\right) d \mathbf{r}_{1}
$$

in which case we obtain

$$
P_{n 1}=\frac{4 f(n, 1)}{\Delta E_{n 1}}\left|\int_{-\infty}^{\infty} \exp \left(i \Delta E_{n 1} t\right) \frac{\mathbf{r}_{2}(t)}{r_{2}^{3}(t)} d t\right|^{2},
$$

where $f(n, 1)$ is the oscillator strength for the $1 \rightarrow n$ transition given by

$$
\frac{1}{3}\left|\int \psi_{n}{ }^{*} \Omega \psi_{1} d \mathbf{r}\right|^{2}=\frac{a_{0}^{2} f(n, 1)}{\Delta E_{n 1}},
$$

$\Delta E_{n 1}$ is the excitation energy in rydberg units, and $r_{2}$ is in units of the Bohr radius $a_{0}$. Remembering that the path of the incident electron can be taken to be a straight line, we have $r_{2}^{2}(t)=\rho^{2}+v^{2} t^{2}$, where $v$ is the speed of the electron. Then the integration occurring in formula (91) can be performed analytically, the resulting expression for $P_{n 1}$ being given in terms of the modified Bessel functions $K_{0}$ and $K_{1}$.

When the coupling between the initial and final states is strong, $P_{n \mathbf{1}}(\rho)$ becomes large for small values of $\rho$ and may even exceed the maximum possible value unity when first-order perturbation theory is employed to evaluate the transition probability. Two different methods can be used to overcome this difficulty:

(i) A cutoff parameter $\rho_{0}$ may be introduced which is independent of electron energy and is chosen so as to give agreement with the Born approximation at high impact energies. Then

$$
Q_{n}=2 \pi \int_{\rho_{0}}^{\infty} P_{n 1}(\rho) \rho d \rho .
$$

(ii) When the coupling is very strong, one can introduce a parameter $\rho_{1}$ chosen so that

$$
P_{n 1}(\rho) \geq \frac{1}{2} \quad\left(\rho \leq \rho_{1}\right),
$$

where $P_{n 1}$ is evaluated using first-order perturbation theory. Since the actual transition probability when the coupling is very strong is an oscillating function of $\rho$ for $\rho<\rho_{1}$, we may replace $P_{n 1}$ by its mean value $\frac{1}{2}$ for $\rho<\rho_{1}$, in which case (86) can be rewritten as

$$
Q_{n}=\frac{1}{2} \pi \rho_{1}^{2}+2 \pi \int_{\rho 1}^{\infty} P_{n 1}(\rho) \rho d \rho .
$$

The procedure employed by Seaton (1962) was to adopt the smaller of the two cross sections obtained using formulas (93) and (95) at each electron energy. The excitation cross section calculated by him for the $1 s \rightarrow 2 p$ transition of hydrogen is displayed in Fig. 13 .

Saraph (1964) has employed essentially the same method as that introduced by Seaton (1962) to evaluate the excitation cross sections $Q_{n, n+1}$ for the $n \rightarrow n+1$ optically allowed transitions of hydrogen. Her calculated values of the quantity $X_{n+1, n}$ arising in the formula

$$
Q_{n, n+1}=X_{n+1, n}{ }^{2} \pi r_{n}^{2},
$$

where $r_{n}=\frac{1}{4}\left(5 n^{2}+1\right) a_{0}$ is the mean radius, are presented in Table XVII. Seaton's impact parameter method is particularly valuable for cases of very strong coupling such as the $n \rightarrow n+1$ optically allowed transitions for large $n$ and the cross sections calculated by Saraph (1964) are to be preferred to those given by the Born approximation for $n \geq 3$. It largely supercedes the modified Bethe approximation discussed in the preceding section.

\subsection{Classical Approximation}

The earliest application of classical mechanics to the inelastic scattering of electrons by atoms was made by Thomson (1912) who regarded the collision as a classical binary impact between the incident electron and one of the atomic electrons which was assumed to be initially at rest. Clearly, at low impact energies, the neglect of the motion of the atomic electron must be an inadequate approximation. Consequently a more refined classical theory was introduced by Gryzinski (1959) making allowance for the velocity of the atomic electron although still treating the collision as an impact between two free electrons, the influence of the nucleus being ignored.

Gryzinski (1959) evaluated the total cross section for the collision of two free electrons, whose initial velocities $\nabla_{1}$ and $\nabla_{2}$ make an angle $\theta$ with each other, such that the energy of the atomic electron changes by an amount $\Delta E$. Then replacing the relative velocity $V$ of the two electrons by the mean value $\bar{V}=\left(v_{1}^{2}+v_{2}^{2}\right)^{1 / 2}$ he integrates over $\theta$ assuming an isotropic velocity distribution for the atomic electron. This yields the total cross section $\sigma(\Delta E)$ for the transfer of an amount of energy $\Delta E$ from the incident electron to the atom. Classically we may suppose that excitation from an initial state 1 to an excited state $n$ of the target atom takes place if $\Delta E$ lies in the range of energies

$$
E_{n}-E_{1} \leq \Delta E<E_{n+1}-E_{1} \text {. }
$$

Integrating over this range of $\Delta E$ therefore gives the classical approximation to the total $1 \rightarrow n$ excitation cross 
section, all final angular momenta of the atom consistent with the specified range of energy transfer being included. It is found that it decays as $E^{-1}$ with the energy $E$ of the incident electron, which is a more rapid fall off than that predicted by quantum theory: $E^{-1}$ In $E$. Stabler (1964) has found a method of integrating over $\theta$ without having to make the approximation of replacing $V$ by $\bar{V}$. This actually leads to somewhat simpler analytical expressions for the total $1 \rightarrow n$ excitation cross section than given by the original Gryzinski evaluation. Another modification of the classical approximation has been introduced by Kingston (1964) who, on the basis of the known slow variation $n^{3} Q_{n}$ with principal quantum number $n$ of the upper level (see the end of Sec. 2.1), expresses the $1 \rightarrow n$ excitation cross section in the form

$$
Q_{n}=n^{-3} \sigma\left(E_{n}-E_{1}\right) \text {. }
$$

In Fig. 17 we display the total cross sections for the $1 \rightarrow 2$ excitation of atomic hydrogen calculated using the various forms of the classical approximation as well as the Born approximation. Due to the rapid fall off with electron energy of the classical approximation cross sections, they are necessarily smaller than the Born approximation cross section at high energies. We see from Fig. 17 that this relationship persists down to low energies for the $1 \rightarrow 2$ transition. However for $n \rightarrow n+1$ transitions with larger values of $n$, the classical approximation cross section becomes larger than the Born approximation cross section at low energies.

In a more recent formulation of the classical scattering problem, Gryzinski (1965a; 1965b; 1965c) employs a continuous distribution of velocities for the atomic electron having the form

$$
f(v)=[(s-2) !]^{-1}\left(v_{0} / v\right)^{s} \exp \left(-v_{0} / v\right) .
$$

The special case $s=3$ gives rise to a total excitation cross section which decays according to the law $E^{-1} \ln E$ for large $E$ in accordance with quantum theory. However it should be noted that the distribution of velocities

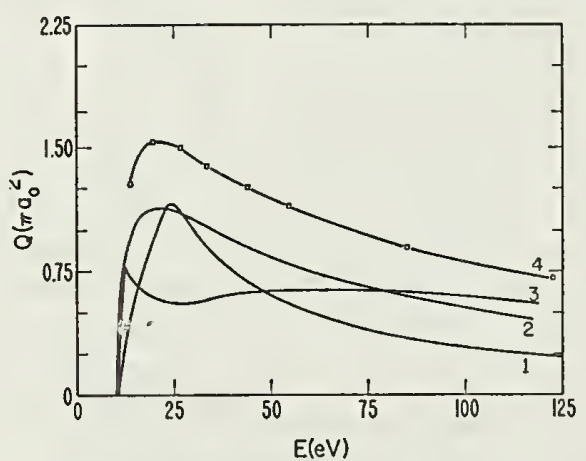

FIG. 17. Total cross sections for the $1 \rightarrow 2$ excitation of atomic hydrogen calculated using various forms of the classical approximation. Curve 1: classical impulse approximation (Stabler, 1964). Curve 2: Gryzinski approximation (Kingston, 1964). Curve 3: semi-classical approximation (Burgess, 1964). Curve 4: first Born approximation (Omidvar, 1965). for the atomic electron given by (99) is quite different from the actual quantum theoretical distribution.

Another attempt at obtaining the correct high-energy behavior of the excitation cross section has been made by Burgess (1964). His approach to the problem was to use the semiclassical impact parameter method described in Sec. 2.10 for impact parameters $\rho>\rho_{0}$ and the classical approximation for $\rho<\rho_{0}$. Taking $V=\bar{V}$ and assuming that the relative probabilities for the excitation of the $2 s$ and $2 p$ states of atomic hydrogen are determined by $\int\left|\psi_{1 s}\right|^{2}\left|\psi_{2 s}\right|^{2} d \mathbf{r}$ and $\int\left|\psi_{1 s}\right|^{2}\left|\psi_{2 p}\right|^{2} d \mathrm{r}$, Burgess (1964) has calculated the $1 s \rightarrow 2 s$ and $1 s \rightarrow 2 p$ excitation cross sections. His cross section for the $1 \rightarrow 2$ transition is displayed in Fig. 17. We note that it exhibits an interesting maximum just above threshold as well as the main maximum at higher electron energies.

A valuable review of classical approximations has been written recently by Burgess and Percival (1968).

\subsection{Vainshtein, Presnyakov, Sobelman Approximation}

A new method which makes explicit allowance for the all important repulsion between the atomic and incident electrons in the wave function characterizing the collision has been introduced by Vainshtein, Presnyakov, and Sobelman (1963).

They express the total wave function in the form

$$
\Psi\left(r_{1}, r_{2}\right)=\psi_{1}\left(r_{1}\right) g\left(r_{1}, r_{2}\right),
$$

where $\psi_{1}$ is the wave function of the initial state of the hydrogen atom, and then substitute into the Schrödinger equation (1). Changing to the new coordinates

$$
R=\frac{1}{2}\left(r_{2}+r_{1}\right), \quad \varrho=\frac{1}{2}\left(r_{2}-r_{1}\right)
$$

and introducing an approximation which is closely equivalent to replacing the interaction $e^{2} / r_{2}$ by $e^{2} / R$, they arrive at the equation

$\left[\frac{\hbar^{2}}{2 m}\left(\nabla_{\mathbf{R}}{ }^{2}+\nabla_{\varrho}{ }^{2}+2 k_{1}{ }^{2}\right)+\frac{e^{2}}{R}-\frac{e^{2}}{\rho}\right] g(\mathbf{R}, \varrho)=0$.

We see that it describes the scattering of two free electrons by each other and the motion of their center of mass in the electrostatic field of the proton. Because the variables $\mathbf{R}$ and $\boldsymbol{\varrho}$ are separated in Eq. (102) its solution $g(\mathbf{R}, \boldsymbol{\varrho})$ can be expressed exactly in terms of confluent hypergeometric functions $F(a, b, z)$. It can be readily verified that

$$
\begin{aligned}
g(\mathbf{R}, \varrho)=N \exp & \left\{i \mathbf{k}_{1} \cdot(\mathbf{R}+\varrho)\right\} \\
& \times F\left(i \nu, 1 ; i k_{1} R-i \mathbf{k}_{1} \cdot \mathbf{R}\right) \\
& \times F\left(-i \nu, 1 ; i k_{1} \rho-i \mathbf{k}_{1} \cdot \varrho\right),
\end{aligned}
$$

where $\nu=k_{1}^{-1}$ and $N=\Gamma(1-i \nu) \Gamma(1+i \nu)$.

Vainshtein, Presnyakov, and Sobelman (1963) now note that the electron-proton interaction $-e^{2} / r_{2}$ gives no contribution to the scattering amplitude for excitation collisions when the Born approximation is used. They therefore neglect this interaction in their own 
approximation and are able, after the application of a peaking approximation, to express the scattering amplitude in the form

$$
f_{n}(K)=-\left(2 m e^{2} / \hbar^{2} K^{2}\right) A \mathscr{g}(1-n),
$$

where $A$ is a dimensionless quantity given by

$$
\begin{aligned}
A=\frac{N K^{2}}{\pi} \int F(i \nu, 1 & \left.; i k_{1} r-i \mathbf{k}_{\mathbf{1}} \cdot \mathbf{r}\right) \\
\times & \times F\left(-i \nu, 1 ; i k_{1} r-i \mathbf{k}_{1} \cdot \mathbf{r}\right) \\
& \times r^{-1} \exp (2 i \mathbf{K} \cdot \mathbf{r}) d \mathbf{r} .
\end{aligned}
$$

We see that if $A$ is set equal to unity we regain the first Born approximation.

Unfortunately the terms which have been neglected in deriving equation (102) give rise to divergences in the limit $k_{1} \rightarrow 0$. By introducing an effective charge $\zeta$, Vainshtein et al. show that these divergences can be eliminated if the parameter $\nu$ is redefined according to the formula

$$
\nu=\left(k_{1}+E_{1}^{1 / 2}\right)^{-1} .
$$

To evaluate $A$, Vainshtein et al. replaced $\exp (2 i \mathrm{~K} \cdot \mathbf{r})$ by exp $(-2 i \mathbb{K} \cdot \mathbf{r})$ in $(105)$. However Omidvar $(1967)$ has discovered that this further approximation is unnecessary and has given a method of evaluating $A$ exactly. Crothers and McCarroll (1965) have introduced a modification of the original approach of Vainshtein $\mathrm{et} \mathrm{al}$. which also enabled them to evaluate $A$ without approximation but they found that the effective charge $\zeta$ and the corresponding value of $\nu$ become complex which seems undesirable.

The cross sections for the $1 s \rightarrow 2 s$ and $1 s \rightarrow 2 p$ excitation calculated by Omidvar using his expression for the scattering amplitude with exchange neglected show subsidiary maxima close to the threshold energy similar to those found by Crothers and McCarroll. We note that a subsidiary maximum close to the threshold was also obtained by Burgess (1964) for the $1 s \rightarrow 2 p$ excitation cross section using a semiclassical approximation which likewise regards the scattering problem as a collision between two free electrons.

There are so many approximations of an uncertain character which are made in the Vainshtein et al. approach, even as treated by Omidvar, that it seems impossible to assess properly the validity of the method. [In a recent note by Crothers (1967) it is claimed that there is an error in the work of Omidvar (1967) arising from an incorrect analytic continuation. Correction of this error leads to the removal of the subsidiary maxima found previously.]

\subsection{Partial Wave Analysis}

At the present stage it is convenient to examine the partial wave analysis in more detail and from a different point of view. The approach we shall follow is that due to Percival and Seaton (1957).
We suppose that the atomic hydrogen electron has principal quantum number $n$ and angular momentum quantum numbers $l_{1}, m_{1}{ }^{l}, m_{1}{ }^{8}$ and that the free electron has wave number $k_{n}$ and angular momentum quantum numbers $l_{2}, m_{2}{ }^{l}, m_{2}{ }^{8}$. The angular momenta of the two electrons may then be coupled together to yield the total orbital angular momentum with quantum numbers $L, M_{L}$ and the total spin angular momentum with quantum numbers $S, M_{S}$. Since the total orbital and spin angular momenta are separately conserved throughout the collision, their quantum numbers remain unaltered.

Introducing the spacial function

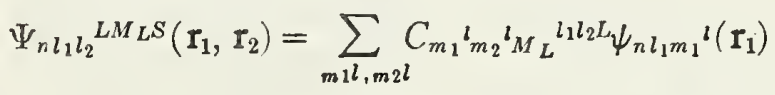

$$
\begin{aligned}
& \times Y_{l_{2} m_{2}}{ }^{2}\left(\theta_{2}, \phi_{2}\right) r_{2}^{-1} F_{n l_{1} l_{2}}{ }^{L S}\left(r_{2}\right),
\end{aligned}
$$

where $C_{m_{1}}{ }^{l} m_{2}{ }^{l} M_{L}{ }^{l_{1} l_{2 L} L}$ is a Clebsch-Gordan vector coupling coefficient, $\psi_{n l_{1} m_{1}}{ }^{l}\left(r_{1}\right)$ is an atomic hydrogen wave function and $Y_{l_{2} m_{2}}{ }^{l}$ is a spherical harmonic, we may expand the complete spacial wave function in the symmetrized forms

$$
\begin{aligned}
\Psi_{\nu} \pm L M_{L} S & \left.\mathbf{r}_{1}, \mathbf{r}_{2}\right)=2^{-1 / 2} \sum_{\nu^{\prime}}\left\{\Psi_{\nu^{\prime}}{ }^{L M L S}\left(\mathbf{r}_{1}, \mathbf{r}_{2}\right)\right. \\
& \left. \pm \Psi_{\nu^{\prime}}{ }^{L M L S}\left(\mathbf{r}_{2}, \mathbf{r}_{1}\right)\right\}
\end{aligned}
$$

where $\nu$ denotes the set of quantum numbers $n l_{1} l_{2}$. The positive sign is associated with the singlet $S=0$ scattering case while the negative sign corresponds to the triplet $S=1$ scattering case. Substituting (108) into the Schrödinger equation (1) then yields the set of coupled integrodifferential equations for the radial functions $F_{\nu}{ }^{L S}(r)$ :

$$
\begin{aligned}
& {\left[\frac{d^{2}}{d r^{2}}+k_{n}{ }^{2}-\frac{l_{2}\left(l_{2}+1\right)}{r^{2}}\right] F_{\nu}{ }^{L S}(r)} \\
& +\left(2 m / \hbar^{2}\right) \sum_{\nu^{\prime}}\left[V_{v v^{\prime}} L(r)-W_{\nu v^{\prime}} L S(r)\right] F_{v^{\prime}} L S(r)=0,
\end{aligned}
$$

where the $V_{\nu \nu}{ }^{L}(r)$ are potential interaction terms and the $W_{\nu v^{\prime}}{ }^{L S}(r)$ are exchange interaction terms whose detailed forms have been given by Percival and Seaton (1957). These equations are independent of $M_{L}$ and $M_{S}$ and exhibit no coupling between states of different $L, S$ quantum numbers.

Let us suppose now that the initial state of the whole system of electron and hydrogen atom is $\nu^{\prime}$ and that the final state is $\nu$. Then the asymptotic behavior of the radial function $F_{\nu}{ }^{L S}(r)$ may be chosen to have the form at large $r$.

$$
\begin{aligned}
F_{\nu}^{L S}(r) \sim k_{n}^{-1 / 2}\left\{\sin \left(k_{n} r-\frac{1}{2} l_{2} \pi\right) \delta_{\nu \nu^{\prime}}\right. \\
+ \\
\left.+\cos \left(k_{n} r-\frac{1}{2} l_{2} \pi\right) R_{\nu \nu^{\prime}}\right\}
\end{aligned}
$$$$
\text { or alternatively }
$$

$$
\begin{aligned}
F_{\nu}{ }_{\nu} S(r) \sim k_{n}{ }^{-1 / 2}\{\exp [ & \left.-i\left(k_{n} r-\frac{1}{2} l_{2} \pi\right)\right] \delta_{\nu v^{\prime}} \\
& \left.-\exp \left[i\left(k_{n} r-\frac{1}{2} l_{2} \pi\right)\right] \delta_{\nu v^{\prime}}\right\},
\end{aligned}
$$


where $R_{\nu \nu^{\prime}}$ and $S_{\nu \nu^{\prime}}$ are the elements of the reactance matrix $\mathrm{R}$ and the scattering matrix $\mathrm{S}$, respectively. It can be readily verified that these matrices are related according to the formula

$$
S=(1+i \mathrm{R}) /(1-i \mathrm{R}) .
$$

Since the reactance matrix is real and symmetric we have

and so

$$
\mathrm{R}^{*}=\mathrm{R}, \quad \widetilde{\mathrm{R}}=\mathrm{R}
$$

$$
\breve{\mathrm{S}}=\mathrm{S}, \quad \mathrm{S} * \mathrm{~S}=1
$$

which means that the scattering matrix is symmetric and unitary. The unitary property of the scattering matrix is equivalent to the conservation theorem of Bohr, Peierls, and Placzek (Mott and Massey, 1965a).

The cross section can be conveniently expressed in terms of the transmission matrix $\mathrm{T}$ defined by the formula

$$
\mathrm{T}=1-\mathrm{S}=-[2 i \mathrm{R} /(1-i \mathrm{R})] .
$$

Thus we have

$Q_{n^{\prime} l_{1^{\prime}}, n l_{1}}=\pi \Omega\left(n l_{1}, n^{\prime} l_{1}^{\prime}\right) /\left[k_{n^{\prime}}{ }^{2} 2\left(2 l_{1}^{\prime}+1\right)\right]$,

where the collision strength $\Omega$ is given by

$$
\begin{aligned}
& \Omega\left(n l_{1}, n^{\prime} l_{1}^{\prime}\right) \\
& \quad=\frac{1}{2} \sum_{l_{2} l_{2}{ }^{\prime} S L}(2 S+1)(2 L+1)\left|T\left(n l_{1} l_{2} S L, n^{\prime} l_{1}^{\prime} l_{2}^{\prime} S L\right)\right|^{2} .
\end{aligned}
$$

\subsection{Unitarized Born Approximation (Sometimes Known as the Born II Approximation)}

The usual Born approximation to the $n^{\prime} l_{1}^{\prime} \rightarrow n l_{1}$ excitation cross section follows from the analysis summarized in the previous section by setting

$$
\mathrm{T}=-2 i \mathrm{R}_{B},
$$

where the matrix $\mathrm{R}_{B}$ is the Born approximation to the $R$ matrix and has elements

$$
\begin{array}{r}
R_{B, v v^{\prime}}=-\frac{2 m}{\hbar^{2}}\left(k_{n} k_{n^{\prime}}\right)^{1 / 2} \int_{0}^{\infty} j_{l_{2}}\left(k_{n} r\right) V_{\nu v^{\prime}}{ }^{L}(r) \\
\times j_{l^{\prime} 2}\left(k_{n^{\prime}} r\right) r^{2} d r .
\end{array}
$$

Expression (118) for the $\mathrm{T}$ matrix corresponds to an $\mathrm{S}$ matrix which does not satisfy the unitary condition $\mathrm{S}$ * $=1$ so that the conservation theorem (Mott and Massey, 1965a) is not obeyed although this is not a serious matter if $\mathrm{T}$ is small.

Seaton (1961) has pointed out that the unitary condition can be satisfied by setting $\mathrm{R}=\mathrm{R}_{B}$ in formula (115) for $\mathrm{T}$ which yields the unitarized Born approximation to the transmission matrix

$$
\mathrm{T}=-2 i \mathrm{R}_{B} /\left(1-i \mathrm{R}_{B}\right) .
$$

Calculation of the Born approximation to the $\mathrm{R}$ matrix for transitions involving the states

$\begin{array}{cccc}\nu & n l_{1} & l_{2} & L \\ 1 & 1 s & \ell & \ell \\ 2 & 2 s & \ell & \ell \\ 3 & 2 p & \ell-1 & \ell \\ 4 & 2 p & \ell+1 & \ell \\ 5 & 2 p & \ell & \ell\end{array}$

have been performed by Lawson, Lawson, and Seaton (1961). Using these matrix elements together with formula (120) for the transmission matrix, V. M. Burke and Seaton (1961) have evaluated the collision strengths $\Omega(1 s, 2 s), \Omega(1 s, 2 p), \Omega(1 s, 2 p 0), \Omega\left(2 s, 2 p_{1 / 2}\right)$, and $\Omega\left(2 s, 2 p_{3 / 2}\right)$. Whereas formula (118) for the transmission matrix makes no allowance for coupling between the initial state or the final state and other states, formula (120) does include some allowance for such couplings. In addition, since the conservation theorem is automatically satisfied by taking the transmission matrix to have the form (120), one might expect the resulting cross sections should be more accurate than those obtained by employing the usual form of the Born approximation. That this is not necessarily so can be verified by inspection of Figure 13 where the cross sections for the $1 s \rightarrow 2 p$ excitation of atomic hydrogen, calculated using the unitarized Born approximation and the $1 s-2 s-2 p$ close coupling approximation with exchange neglected, are compared. The inadequacy of the unitarized Born approximation for the $1 s \rightarrow 2 s$ and $1 s \rightarrow 2 p$ excitations occurs because the couplings involved are insufficiently strong.

Coupling to the $3 s, 3 p$, and $3 d$ states has been included in an investigation carried out by Somerville $(1962 ; 1963)$ using the unitarized Born approximation but the resulting changes in the cross sections were small.

\subsection{Close Coupling Approximation}

A much more satisfactory approach to the problem of determining excitation cross sections is to obtain the transmission matrix elements by means of the exact solution of the coupled integrodifferential equations (109) using numerical techniques. Since one cannot solve an infinite set of coupled equations, the atomic states which enter into the expansion of the total wave function (107) are confined to just a few of the lowestlying states giving rise to a finite set of coupled integrodifferential equations. This is called a close coupling approximation, the order of the approximation depending upon the atomic states which are retained in the expansion of the total wave function.

The first calculations along these lines were concerned with the $1 s \rightarrow 2 s$ excitation and neglected coupling to all states other than the initial and final states. We refer to this as the $1 s-2 s$ close coupling approximation. Bransden and McKee (1956) performed a numerical integration of the pair of coupled equations associated 
with the $\ell=0$ partial wave in which exchange was neglected. Subsequently Marriott (1958) performed a similar investigation but included full allowance for exchange. The resulting values of the $1 s \rightarrow 2 s$ excitation cross sections for the zero-order partial wave, $Q_{28}{ }^{0}$ and $Q_{28}{ }^{0 \pm}$, where the absence of a sign indicates exchange neglected and the positive and negative signs denote singlet and triplet scattering, respectively, are given in Table XIV. As we have noted earlier, when exchange is neglected, the first Born and the distorted waves approximations both provide partial cross sections $Q_{28}{ }^{0}$ which are in fairly satisfactory agreement with the results of the numerical integration of the coupled differential equations. However, when exchange is included, the Born-Oppenheimer and the distorted waves approximations give rise to partial cross sections $Q_{28}{ }^{0 \pm}$ which are considerably different from those calculated using the $1 s-2 s$ close coupling approximation.

The calculations of Bransden and McKee (1956), in which exchange was neglected, were then extended by Smith, Miller, and Mumford (1960) to all partial waves making a significant contribution to the total $1 s \rightarrow 2 s$ excitation cross section for electron impact energies up to $54 \mathrm{eV}$ their cross-section curve being displayed in Fig. 18. They also included coupling to the $3 s$ state, which was found to have the effect of slightly reducing the total $1 s \rightarrow 2 s$ excitation cross sections; in addition they calculated the $1 s \rightarrow 3 s$ excitation cross section using the $1 s-3 s$ and the $1 s-2 s-3 s$ close coupling approximations. Subsequently Smith (1960) extended the calculations of Marriott (1958) on the $1 s \rightarrow 2 s$ excitation, in which exchange was taken into account, to the $l=1$ and 2 partial waves.

Referring to the list of states (121) we note that the states $\nu=1,2,3,4$ are of the same parity and lead to four coupled integro-differential equations for $L>0$ and to three coupled equations for $L=0$ because the $\nu=3$ state ceases to exist for this special case. Since the $\nu=5$ state has a different parity to the other states, it is uncoupled to them and gives rise to a separate equation. The solution of this equation is required only for elastic scattering and for transitions between the $2 p$ states with $j=\frac{1}{2}$ and $j=\frac{3}{2}$.

These coupled integrodifferential equations have been solved numerically by several investigators for a number of values of $L$ (P. G. Burke, V. M. Burke, Percival, and McCarroll, 1962; V. M. Burke and McCarroll, 1962; Damburg, 1962; Damburg and Peterkop, 1962 a, b, c and 1963; Burke, Schey, and Smith, 1963; Gailitis and Damburg, 1963; Damburg and Gailitis, 1963, Burke, Ormonde, and Whitaker, 1966; Burke, Ormonde, Taylor, and Whitaker, 1967; and Omidvar, 1964). At the time of writing the most extensive calculations have been carried out by Burke, Schey, and Smith (1963) and by Omidvar (1964). In Tables XVIII and XIX are displayed the partial cross sections $Q^{L}$ for the $1 s \rightarrow 2 s$ and $1 s \rightarrow 2 p$ excitations of atomic hydrogen calculated by Burke, Schey, and Smith (1963) according to the $1 s-2 s-2 p$ close coupling approximation with allowance made for exchange in which all four integrodifferential equations connecting the $\nu=1,2,3,4$ states are solved for each value of $L$. These values of the singlet and triplet partial cross sections include the spin weighting factors $\frac{1}{4}$ and $\frac{3}{4}$, respectively. All $L$ values making a significant contribution to the total cross sections were included. Presented in Tables XX and $\mathrm{XXI}$ are the total $1 s \rightarrow 2 s$ and $1 s \rightarrow 2 p$ excitation cross sections calculated by Burke, Schey, and Srnith (1963) employing the $1 s-2 s-2 p$ close coupling approximation with exchange taken into account. It is instructive to compare these total excitation cross sections with those calculated by Omidvar (1964) according to the $1 s-2 s-2 p$ close coupling approximation with exchange neglected. This comparison is made in Figs. 18 and 19; we see that the effect of allowing for exchange is to reduce substantially both the $1 s \rightarrow 2 s$ and $1 s \rightarrow 2 p$ excitation cross sections close to the threshold energy although the behavior becomes more complicated a few volts above threshold for the $1 s \rightarrow 2 s$ excitation. To show the effect of coupling to states other than the initial and final states of the transitions, we also display in Figs. 18 and 19 the total $1 s \rightarrow 2 s$ excitation cross section calculated using the $1 s-2 s$ close coupling approximation and the total $1 s \rightarrow 2 p$ excitation cross section calculated according to the $1 s-2 p$ close coupling approximation, exchange being included in both cases. We see that coupling to the states different from the initial and final states of the transition causes a reduction in the excitation cross sections, except for the $1 s \rightarrow 2 s$ excitation close to the threshold energy where in fact the coupling to the $2 p$ state produces a small increase.

Because of the probable importance of coupling to higher states, Burke (1963) investigated the convergence of the close coupling approximation by solving the seven coupled integrodifferential equations involved in the $1 s-2 s-2 p-3 s-3 p$ close coupling approximation

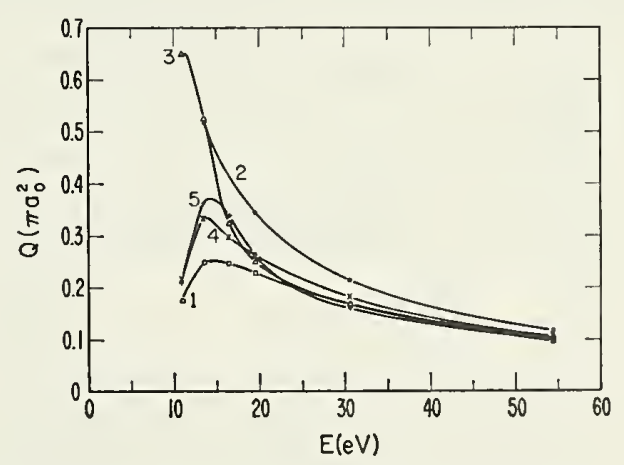

Fic. 18. Total cross sections for the $1 s \rightarrow 2 s$ excitation of atomic hydrogen. Curve 1: first Born approximation (Omidvar, 1964). Curve 2: $1 s-2 s$ close coupling approximation neglecting exchange (Smith, Miller, and Mumford, 1960). Curve 3: $1 s-2 s-2 p$ close coupling approximation neglecting exchange (Omidvar, 1964). Curve 4: $1 s-2 s$ close coupling approximation including exchange (Omidvar, 1964). Curve 5: $1 s-2 s-2 p$ close coupling approximation including exchange (Burke, Schey, and Smith, 1963). 
at $16.5 \mathrm{eV}$. The results of his calculations for the $1 s \rightarrow 2 s$, $1 s \rightarrow 2 p$, and $1 s \rightarrow 3 p$ excitation cross sections are displayed in Table XXII. Clearly the effect of coupling to the $3 s$ and $3 p$ states reduces the cross sections substantially, particularly for the $1 s \rightarrow 2 s$ excitation case. It is to be anticipated that the still higher states will have a profound effect upon the total cross sections.

Burke, Schey, and Smith (1963) have also calculated the $1 s \rightarrow 3 p$ excitation cross section using the $1 s-3 p$ close coupling approximation. They find a large peak in the neighborhood of $15 \mathrm{eV}$ as can be seen from Fig. 20 where their cross-section curve is compared with the Born approximation. However the $1 s-2 s-2 p-3 s-3 p$ close coupling calculation performed by Burke (1963) shows that coupling to the $2 s, 2 p$, and $3 s$ states is important, reducing the $1 s \rightarrow 3 p$ excitation cross section from 0.319 $\pi a_{0}^{2}$ to $0.250 \pi a_{0}^{2}$ at $16.5 \mathrm{eV}$. Coupling to higher states may well be important also.

Very interesting calculations in the neighborhood of the threshold energy for the excitation of the $2 s$ and $2 p$ states of atomic hydrogen have been performed by Damburg and Gailitis (1963) and Gailitis and Damburg (1963). They employed the $1 s-2 s-2 p$ close coupling approximation with exchange included to determine the $1 s \rightarrow 2 s$ and $1 s \rightarrow 2 p$ partial cross sections for $L=0.1,2$. These are displayed in Table XXIII. Using the $1 s-2 s-2 p$ close coupling approximation with exchange neglected, they also calculated the partial cross sections for $L \geq 3$ so obtaining the total cross sections for the $1 s \rightarrow 2 s$ and $1 s \rightarrow 2 p$ excitations given in Table XXIV. We see that the $L=0,1,2$ partial cross sections as well as the total cross sections for the $1 s \rightarrow 2 s$ and $1 s \rightarrow 2 p$ excitations remain finite in the vicinity of the threshold energy. This behavior can be understood by virtue of the following considerations. The effect of the close coupling between the degenerate $2 s$ and $2 p$ states is to introduce an additional potential $-\alpha / r^{2}$ acting upon the free electron, where $\alpha$ is a symmetrical matrix whose diagonal elements are zero. The centrifugal term $l_{2}\left(l_{2}+1\right) / r^{2}$ occurring in the radial equation (109) then

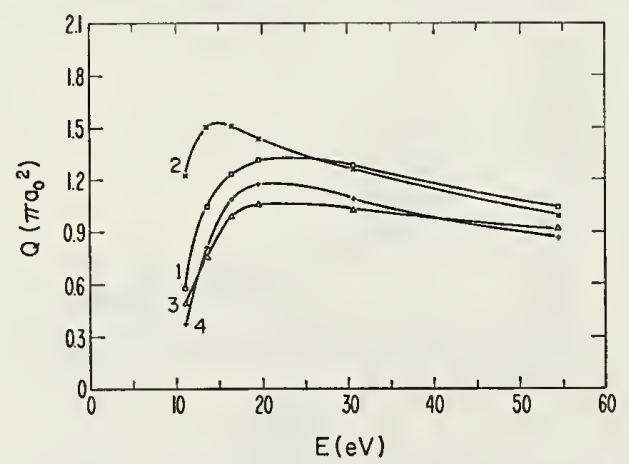

FIG. 19. Total cross sections for the $1 s \rightarrow 2 p$ excitation of atomic hydrogen. Curve 1: first Born approximation (Omidvar, 1964). Curve 2: $1 s-2 s-2 p$ close coupling approximation neglecting exchange (Omidvar, 1964). Curve $3: 1 s-2 p$ close coupling approximation including exchange (Omidvar, 1964). Curve 4: $1 s-2 s-2 p$ close coupling approximation including exchange (Burke, Schey, and Smith, 1963).

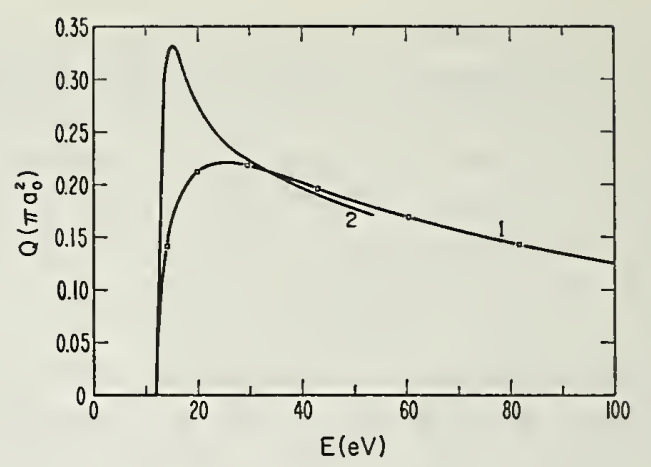

Fic. 20. Total cross sections for the $1 s \rightarrow 3 p$ excitation of atomic hydrogen. Curve 1: first Born approximation (Vainshtein, 1965). Curve 2: $1 s-3 p$ close coupling approximation including exchange (Burke, Schey, and Smith, 1963).

becomes replaced by $\lambda(\lambda+1) / r^{2}$, where $a=\lambda(\lambda+1)$ is a characteristic root obtained by diagonalizing the matrix $l_{2}\left(l_{2}+1\right)-\alpha$ so that

$$
\lambda=-\frac{1}{2}+\left(\frac{1}{4}+a\right)^{2} .
$$

The threshold behavior of $\left|T\left(\nu, \nu^{\prime}\right)\right|^{2}$, where $\mathrm{T}$ is the transmission matrix, is given by $\left|k_{2}^{\lambda+1 / 2}\right|^{2}$ so that provided $\lambda+\frac{1}{2}$ is real the partial cross section for the $\nu^{\prime} \rightarrow \nu$ excitation vanishes in the limit as $k_{2} \rightarrow 0$ in the usual way. However if there are negative values of $a<-\frac{1}{4}$ then $\lambda+\frac{1}{2}=i \mu$, where $\mu$ is real and so

$$
\left|k_{2}{ }^{\lambda+1 / 2}\right|^{2}=\left|k_{2}^{i \mu}\right|^{2}=1
$$

which means that the excitation cross section remains finite in the neighborhood of the threshold.

An extension of the above argument shows also that the cross sections oscillate in the vicinity of the threshold, this being confirmed by the $1 s-2 s-2 p$ close coupling calculations performed by Damburg and Gailitis (1963) which reveal that the $1 s \rightarrow 2 p$ excitation cross section exhibits a minimum just above the threshold energy.

Very lengthy computations on the behavior of the $1 s \rightarrow 2 s$ and $1 s \rightarrow 2 p$ excitation cross sections in the vicinity of the $n=2$ threshold employing the 6 state $1 s-2 s-2 p-3 s-3 p-3 d$ close coupling approximation have been carried out recently by Burke, Ormonde, and Whitaker $(1966,1967)$. Their cross sections are compared with those obtained using the 3 -state $1 s-2 s-2 p$ close coupling approximation in Figs. 21 and 22. The effect of adding a number of correlation terms to the 3 state close coupling expansion has been investigated by Burke and Taylor (1966) and more extensively by Taylor and Burke (1967). The results obtained with this correlation approximation employing 20 correlation terms, which allow explicitly for the interelectron separation $r_{12}$ in a similar fashion to that done in bound state problems, are also displayed in Figs. 21 and 22.

Just above the $n=2$ threshold at $k_{1}{ }^{2}=0.75$ the 3 -state approximation, with and without allowance for correlation, is dominated by an $S=0, L=1$ resonance which presumably would also be found using the 6-state ap- 
proximation if extended to sufficiently low energies. Above $k_{1}{ }^{2}=0.85$ the excitation cross sections given by the 6-state approximation are dominated by resonances arising from the $n=3$ level.

An interesting feature of the three calculations whose results are presented in Figs. 21 and 22 is their satisfactory accordance over the energy range from just above the $n=2$ threshold to $k_{1}{ }^{2}=0.85$. This has led Burke and his collaborators to make the hypothesis that the close coupling approximation is valid for all energies which are insufficient to excite resonances and channels associated with the lowest level excluded from the expansion. In particular they suggest that the 6state approximation should be quite accurate for all energies below that required to excite the resonances arising from the $n=4$ level, that is below about $12.5 \mathrm{eV}$.

\section{Helium}

Having examined the various approximations which have been used to investigate the excitation of atomic hydrogen, we now direct our attention to their application to the study of atomic systems with more than one electron. The simplest of these is helium which is a particularly interesting case to treat in detail since it has been the subject of the most thorough experimental investigation.

\subsection{First Born Approximation}

As for the case of atomic hydrogen we begin by looking at the Born approximation. The earliest calculations using this approximation were performed by Massey and Mohr (1931; 1933b) who applied it to the excitation of a number of singlet states of helium. In the treatment of helium excitation we need to use approximate wave functions to describe the atomic electrons. For the ground state of the helium atom Massey and Mohr chose the very simple Hylleraas (1929) function

where

$$
\psi_{1}\left(r_{1}, r_{2}\right)=\phi_{1}\left(Z \mid r_{1}\right) \phi_{1}\left(Z \mid r_{2}\right),
$$

$$
\phi_{1}(\lambda \mid r)=\left(\lambda^{3} / \pi\right)^{1 / 2} \exp (-\lambda r)
$$

the parameter $\lambda$ being determined by the variational method to be $Z=1.6875$. (We express radial distances in units of the Bohr radius $a_{0}$.) To represent the excited states of helium they used symmetrized functions composed of products of hydrogenic orbitals.

Because of the approximate nature of the helium atom wave functions there is an additional uncertainty in these calculations beyond that arising from the application of the Born approximation. Bates, Fundaminsky, Leech, and Massey (1950) have pointed out that information regarding the error introduced by the use of approximate wave functions can be obtained by employing an alternative expression for the scattering amplitude which weights the various regions of coordinate space differently. We have already shown that for the excitation of the $n$th state of a hydrogen atom from the ground state, the first Born approximation to the scattcring amplitude is given by

$$
f_{n} \mathbf{x}(K)=\frac{-2 m e^{2}}{\hbar^{2} K^{2}} \int \exp (i K z) \psi_{n}^{*}(\mathbf{r}) \psi_{1}(\mathbf{r}) d \mathbf{r},
$$

where the $z$ axis has been chosen along the momentum change vector $\hbar \mathrm{K}$. If we now make use of the Schrödinger equations satisfied by the exact wave functions $\psi_{1}$ and $\psi_{n}$ of the initial and final states of the atom, we find that this expression can be replaced by the alternative formula:

$$
\begin{aligned}
& f_{n} \mathrm{II}(K)=-i \frac{e^{2}}{K\left(E_{1}-E_{n}\right)} \int \exp (i K z) \\
& \times\left\{\psi_{n} * \frac{\partial}{\partial z} \psi_{1}-\psi_{1} \frac{\partial}{\partial z} \psi_{n} *\right\} d \mathrm{r} .
\end{aligned}
$$

Calculations have been performed by Altshuler (1952; $1953)$ on the $1^{1} S \rightarrow 2^{1} P, 1^{1} S \rightarrow 3^{1} P$, and $1^{1} S \rightarrow 2^{1} S$ excitations of helium using both forms for the scattering amplitude, suitably modified to hold for the case of two electron atomic systems. To represent the ground state of helium he took the simple Hylleraas function (122) while for the $n^{1} P$ states of helium he chose

$$
\begin{aligned}
\psi_{n l m}\left(\mathrm{r}_{1}, \mathrm{r}_{2}\right)=2^{-1 / 2}\left\{\phi_{1}\left(2 \mid r_{1}\right) \phi_{n l m}\left(1 \mid \mathrm{r}_{2}\right)\right. \\
\left.+\phi_{1}\left(2 \mid r_{2}\right) \phi_{n l m}\left(1 \mid \mathrm{r}_{1}\right)\right\},
\end{aligned}
$$

where $\phi_{n l m}(\lambda \mid \mathbf{r})$ is the hydrogenic wave function for the state with quantum numbers $n, l, m$ corresponding to an effective charge $\lambda$. For the $2^{1} S$ state of helium the function (126) is inadequate because it is not orthogonal to the ground-state wave function $\psi_{1}$. However this orthogonalization was achieved by taking

$$
\begin{aligned}
\psi_{200}\left(r_{1}, r_{2}\right)= & 2^{-1 / 2}\left\{\phi_{1}\left(\alpha \mid r_{1}\right) \phi_{200}\left(\beta \mid r_{2}\right)\right. \\
& \left.+\phi_{1}\left(\alpha \mid r_{2}\right) \phi_{200}\left(\beta \mid r_{1}\right)\right\}+A \psi_{1}\left(r_{1}, r_{2}\right),
\end{aligned}
$$

where $A$ was chosen so as to orthogonalize $\psi_{200}$ and $\psi_{1}$ while the screening parameters were determined by the variational method to be $\alpha=1.98, \beta=1.20$. The total excitation cross sections obtained by Altshuler are displayed in Figs. 23, 24, and 25. We see that although the absolute magnitudes of the total excitation cross sections calculated with the aid of formulas I and II differ somewhat, their dependence upon energy are rather similar.

The helium wave functions (122) and (126) give rise to the very poor value 0.187 for the $1^{1} S \rightarrow 2^{1} P$ oscillator strength compared to the value 0.266 calculated by Wheeler (1933) using an accurate wave function for the ground state of helium obtained by Hylleraas (1930) as well as an improved wave function for the $2^{1} P$ state. Lassettre and Jones (1964) have calculated the differential cross section for the $1^{1} S \rightarrow 2^{1} P$ excitation of helium employing the same atomic wave functions as Wheeler. An interesting feature of their calculations, first pointed out by Miller and Platzmann 
(1957), is that although there is very poor accordance between the differential cross section calculated by Lassettre and Jones using the accurate wave functions of Wheeler and that calculated using the crude hydrogen-like functions (122) and (126) together with formula I for the scattering amplitude, there is satisfactory agreement between the refined calculations of Lassettre and Jones and the differential cross section calculated by Altshuler (1952) using formula II and the hydrogen-like functions. The calculations of Lassettre and Jones were extended to larger values of $K^{2}$ by Silverman and Lassettre (1964) who compared them with their experimental data for $500-\mathrm{eV}$ electrons. It can be seen from Fig. 26 that the agreement between the shape of the theoretical curve and the experimental data is remarkably good.

Fox (1965) has also used the Born approximation to calculate cross sections for the $1^{1} S \rightarrow n^{1} S$ excitations of helium corresponding to $n=2, \cdots, 10$. For the groundstate wave function he chose the Hartree self-consistent field function given by

$$
\psi_{1}\left(r_{1}, r_{2}\right)=\phi_{1}\left(r_{1}\right) \phi_{1}\left(r_{2}\right),
$$

fitting the one-electron orbital $\phi_{1}$ to a sum of two exponential terms, while for the excited $n^{1} S$ state of helium Fox took wave functions having the form $\psi_{n}+A \psi_{1}$, where $A$ is a constant chosen so as to orthogonalize against $\psi_{1}$, and

$$
\begin{aligned}
\psi_{n}\left(r_{1}, r_{2}\right)=2^{-1 / 2}\left\{\phi_{1}\left(2 \mid r_{1}\right) \phi_{n}\left(r_{2}\right)\right. & \\
& \left.+\phi_{1}\left(2 \mid r_{2}\right) \phi_{n}\left(r_{1}\right)\right\},
\end{aligned}
$$

where $\phi_{r}$ is a truncated Whittaker function. In Fig. 27 we compare the differential cross section for the $1^{1} S \rightarrow 2^{1} S$ excitation calculated by Fox (1965) using the Born approximation with the experimental data of Lassettre, Krasnow, and Silverman (1964) for $500-\mathrm{eV}$ electrons. The accordance is very satisfactory. The total cross sections for the excitation of the $n^{1} S$ states of

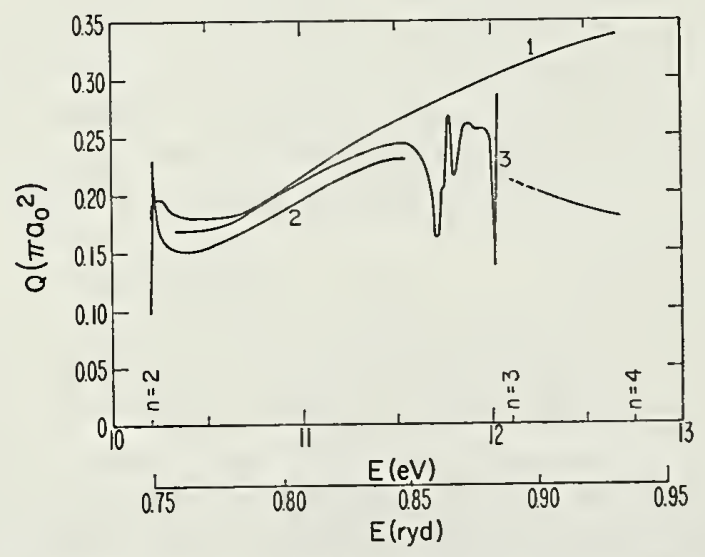

FIG. 21. Total cross sections for the $1 s \rightarrow 2 s$ excitation of atomic hydrogen (Burke, Ormonde, Taylor, and Whitaker, 1967). Curve 1: $1 s-2 s-2 p$ close coupling approximation. Curve 2: correlation approximation. Curve $3: 1 s-2 s-2 p-3 s-3 p-3 d$ close coupling approximation.

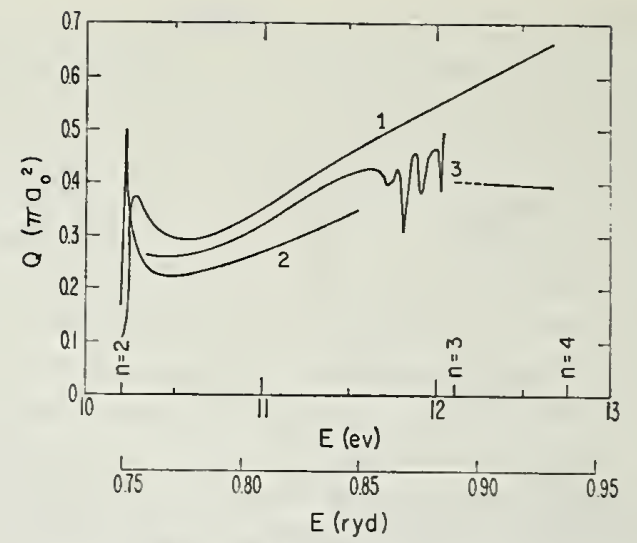

FIG. 22. Total cross sections for the $1 s \rightarrow 2 p$ excitation of atomic hydrogen (Burke, Ormonde, Taylor, and Whitaker 1967). Curve 1: $1 s-2 s-2 p$ close coupling approximation. Curve 2: correlation approximation. Curve $3: 1 s-2 s-2 p-3 s-3 p-3 d$ close coupling approximation.

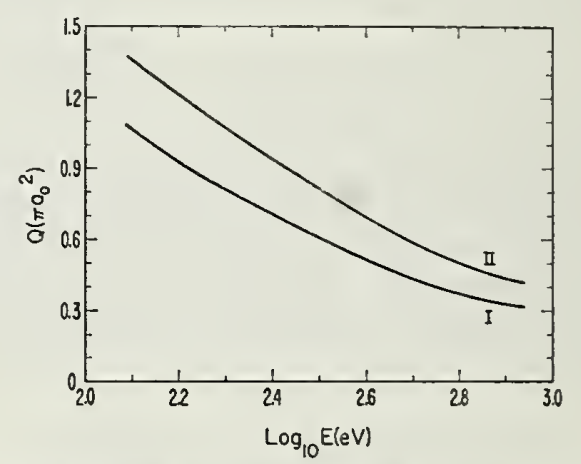

FIg. 23. Total cross sections for the $1^{1} S \rightarrow 2^{1} P$ excitation of helium calculated using formulas I and II for the first Born approximation to the scattering amplitude (Altshuler, 1952).

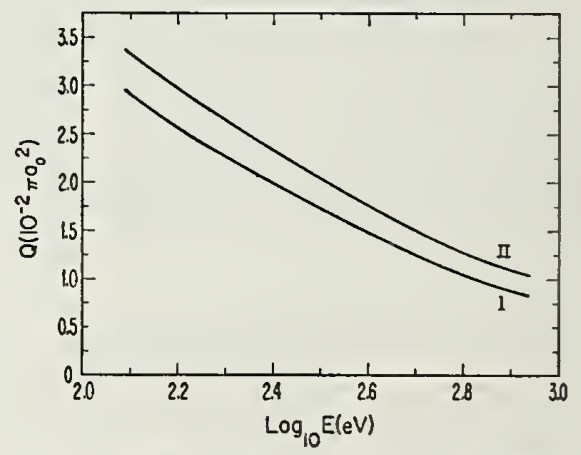

FIG. 24. Total cross sections for the $1^{1} S \rightarrow 3^{1} P$ excitation of helium calculated using formulas I and II for the first Born approximation to the scattering amplitude (Altshuler, 1952).

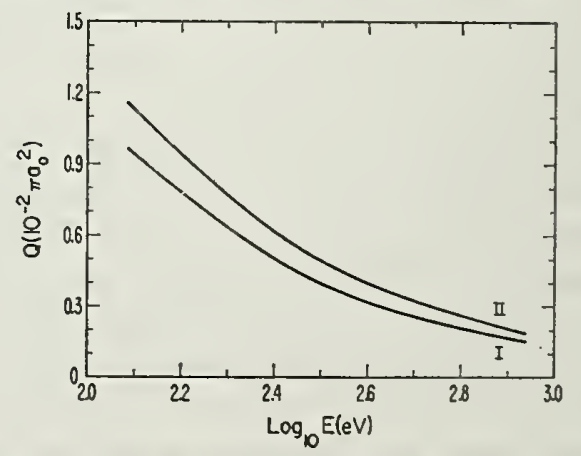

FIG. 25. Total cross sections for the $1^{1} S \rightarrow 2^{1} S$ excitation of helium calculated using formulas I and II for the first Born approximation to the scattering amplitude (Altshuler, 1952). 


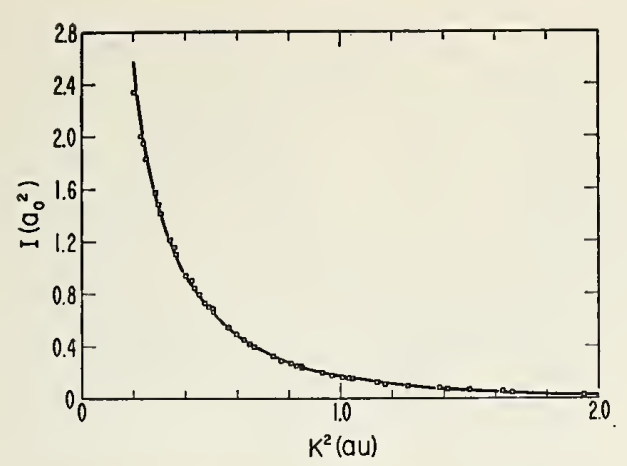

FIG. 26. Differential cross section for the $1^{1} S \rightarrow 2^{1} P$ excitation of helium at $500 \mathrm{eV}$. Solid curve: first Born approximation (Lassettre and Jones, 1964; Silverman and Lassettre, 1964). $\square$ symbols: experimental data for approximately $500 \mathrm{eV}$ (Silverman and Lassettre, 1964).

helium for $n=2, \cdots, 10$ calculated by Fox (1965) are displayed in Fig. 28.

Fox $(1966 ; 1967)$ has also investigated the $1^{1} S \rightarrow 3^{1} D$ excitation of helium using the Born approximation, taking the Eckhart (1930) function for the ground state of helium given by

$$
\begin{aligned}
\psi_{1}\left(r_{1}, r_{2}\right)=\exp \left\{-\left(\alpha r_{1}+\beta r_{2}\right)\right\} & \\
& +\exp \left\{-\left(\alpha r_{2}+\beta r_{1}\right)\right\}
\end{aligned}
$$

with $\alpha=2.14, \beta=1.19$ and the function (126) for the ${ }^{1} D$ state of helium. He obtained the total excitation cross section displayed in Fig. 29 employing the standard form I of the Born approximation and the alternative form II. In the same way as for the excitations of the $2^{1} S, 2^{1} P$, and $3^{1} P$ states of helium treated by Altshuler $(1952$; 1953), the alternative form of the Born approximation gives rise to the larger cross section. By calculating the scattering in the forward direction, Fox (1966) shows further that the Eckhart ground-state helium wave function produces a cross section which is larger than that resulting from the use of a Hartree self-consistant field function for the ground state.

The Born approximation has also been used by Moiseiwitsch (1957) to calculate cross sections for the $2^{3} S \rightarrow 2^{3} P, 3^{3} P, 3^{3} D$, and $4^{3} D$ excitations of helium, the wave functions being chosen to have the general form

$\psi_{n l m}\left(\mathrm{I}_{1}, \mathrm{r}_{2}\right)=2^{-1 / 2}\left\{\phi_{1}\left(2 \mid r_{1}\right) \phi_{n l m}\left(\mathrm{I}_{2}\right)\right.$

$$
\left.-\phi_{1}\left(2 \mid r_{2}\right) \phi_{n l m}\left(\mathrm{r}_{1}\right)\right\} \text {. }
$$

For the $2^{3} S \rightarrow 2^{3} P, 3^{3} D$, and $4^{3} D$ excitations, the helium $2 s$ orbital was chosen to be

$$
\phi_{2 s}(r)=N\left\{1-\frac{1}{3}(\alpha+\beta) r\right\} e^{-\beta r}
$$

with $\alpha=2.00, \beta=0.695$ while $\phi_{2 p m}, \phi_{3 d m}$, and $\phi_{4 d m}$ were assumed to be hydrogenic. The $2^{3} S \rightarrow 3^{3} P$ excitation cross section was found to be very sensitive to the parameters of both the initial and final orbitals. Consequently, for this particular case, use was made of the more accurate atomic orbitals derived by Morse, Young, and Haurwitz (1935) and by Goldberg and Clogston (1939):

$$
\begin{gathered}
\phi_{28}(r)=N\left(r e^{-\mu r}-A e^{-a r}\right) \\
\phi_{3 p m}(\mathbf{r})=M\left(r e^{-\lambda r}-B e^{-b r}\right) r\left\{\begin{array}{l}
\cos \theta \\
(1 / \sqrt{2}) \sin \theta e^{ \pm i \phi} .
\end{array}\right.
\end{gathered}
$$

The results of these Born approximation calculations are displayed in Table XXV.

In the case of the $2^{3} S \rightarrow 2^{3} P$ excitation, the coupling between the initial and final states is rather strong. For this reason the Seaton modification of the Bethe approximation (1961) was also used by Moiseiwitsch (1957) to calculate the total cross section for the $2^{3} S \rightarrow 2^{3} P$ transition, the results being compared with those given by the Born approximation in Table $\mathrm{XXV}$. We see that the maximum value of the cross section is reduced from the Born approximation value of nearly $300 \pi a_{0}{ }^{2}$ to about $115 \pi a_{0}{ }^{2}$, the position of the maximum being displaced to higher electron impact energies and the shape of the peak being altered substantially.

\subsection{Exchange Approximations}

The approximations which we are dealing with under the present heading are the Born-Oppenheimer approximation, the first-order exchange approximation, and the Ochkur approximation.

One of the earliest investigations using the BornOppenheimer approximation was concerned with the $1^{1} S \rightarrow 2^{3} S, 2^{3} P, 3^{3} P, 3^{3} D$, and $4^{3} P$ excitations and was carried out by Massey and Mohr (1931; 1933b). These transitions all involve a change in the total spin of the helium atom and can only occur as the result of electron exchange. The exchange scattering amplitude was obtained by expanding in a series of spherical harmonics. Their calculations were for 100-, $200-$, and $400-\mathrm{eV}$ incident electrons where the cross sections are all very small. Further calculations were performed by Bates et al. (1950) who, in particular, investigated the difference in the cross sections arising from using the post and prior interaction forms of the Born-Oppenheimer approximation, often referred to as the post-prior discrepancy. The prior and post interactions are those between the incident electron and the atom, and between the scattered electron and the atom, respectively. If an exchange of electrons has taken place, these two interactions will be different. However, the scattering amplitudes given by the Born-Oppenheimer approximation associated with the post and prior forms of interaction are still the same provided exact atomic wave functions are employed which means that for the case of scattering by atomic hydrogen there is no post-prior discrepancy. On the other hand, since we have to use approximate atomic wave functions for scattering by helium, the two forms of BornOppenheimer approximation will lead to different results in this case. This is illustrated in Fig. 30 where the 


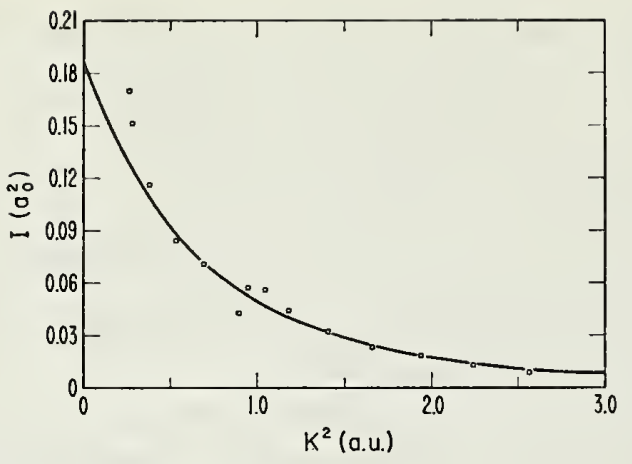

FIG. 27. Differential cross section for the $1^{1} S \rightarrow 2^{1} S$ excitation of helium at $500 \mathrm{eV}$. Solid curve: first Born approximation (Fox, 1965). $\square$ symbols: experimental data for approximately $500 \mathrm{eV}$ (Lassettre, Krasnow, and Silverman, 1964).

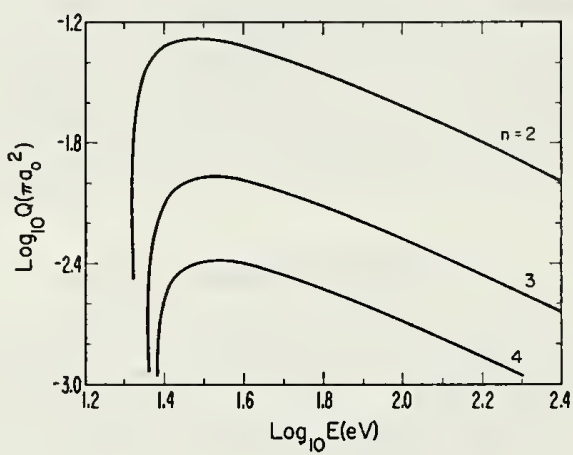

(a)

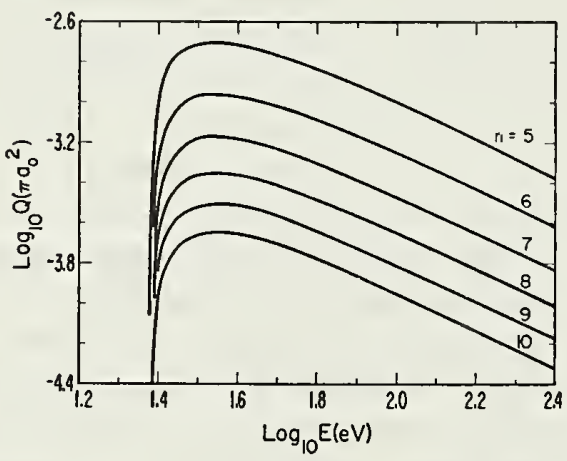

Fig. 28. Total cross sections for the $1^{1} S \rightarrow n^{1} S$ excitations of helium for $n=2,3, \cdots, 10$ calculated using the first Born approximation (Fox, 1965).

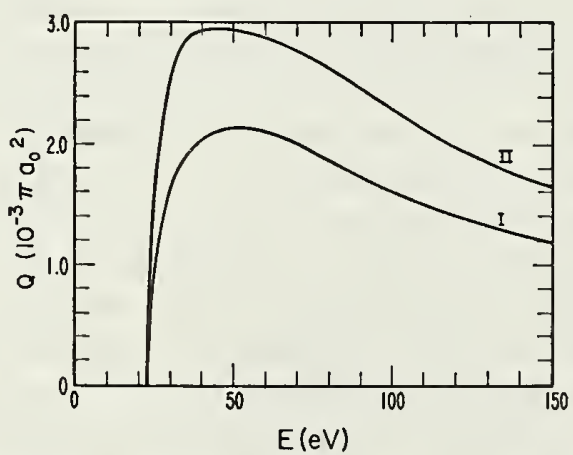

Fig. 29. Total cross sections for the $1^{1} S \rightarrow 3^{1} D$ excitation of helium calculated using formulas I and II for the first Born approximation to the scattering amplitude (Fox, 1966). (a)

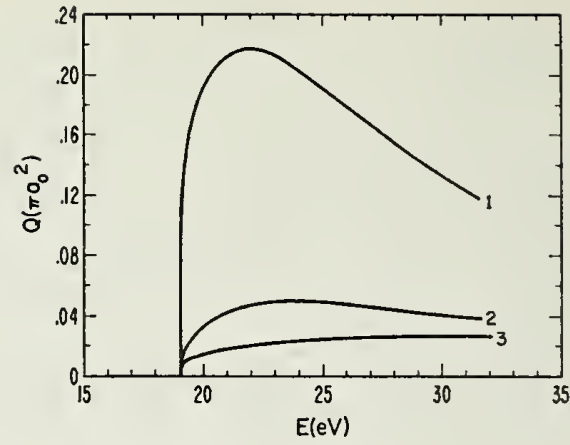

(b)

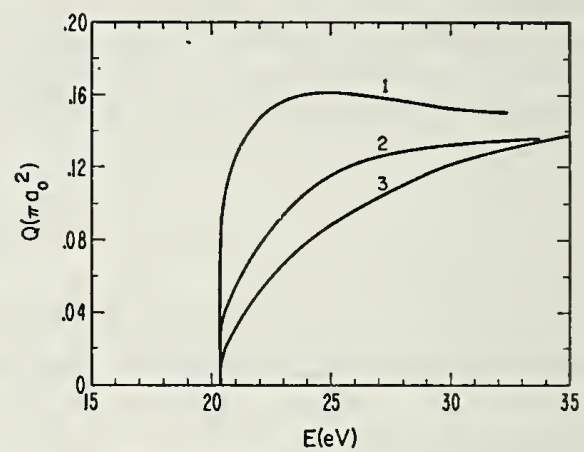

FIG. 30. Total cross sections for the excitation of the $2^{1} S$ and $2^{1} P$ states of helium $\left(\mathrm{a}-2^{1} S, \mathrm{~b}-2^{1} P\right)$ (Bates, Fundaminsky, Leech, and Massey, 1950). Curve 1: Born-Oppenheimer approximation using post interaction. Curve 2: Born-Oppenheimer approximation using prior interaction. Curve 3: first Born approximation.

total cross sections for the $1^{1} S \rightarrow 2^{1} S$ and $1^{1} S \rightarrow 2^{1} P$ excitations of helium calculated using the post and prior forms of the Born-Oppenheimer approximation are displayed. We see that the discrepancy is very great for the $S \rightarrow S$ transition near the threshold but relatively small for the $S \rightarrow P$ transition. This is in accordance with the observation made previously that the Born-Oppenheimer approximation is very poor for $S \rightarrow S$ transitions, typically giving rise to greatly overestimated cross sections.

More recent calculations using the Born-Oppenheimer approximation have been carried out by Massey and Moiseiwitsch $(1954 ; 1960)$ and by Bell, Eissa, and Moiseiwitsch (1966) for the excitation of the $2^{3} S$ and $2^{3} P$ states of helium. The calculations performed by Massey and Moiseiwitsch used the simple Hylleraas ground-state helium wave function (122) while those carried out by Bell, Eissa, and Moiseiwitsch used the variational function obtained by Green, Mulder, Lewis, and Woll (1954) which has the form (128) with

$$
\phi_{1}(r)=N\left(e^{-Z r}+c e^{-2 Z r}\right)
$$

and $Z=1.4558, c=0.6$. This is a rather good analytical fit to the Hartree self-consistent field function for the ground state of helium. Both groups of investigators employed the wave functions for the $2^{3} S$ and $2^{8} P$ states obtained by Morse, Young, and Haurwitz (1935) possessing the form (131) with $\phi_{2 s}$ being given by formula (133) and $\phi_{2 p m}$ being a hydrogen-like orbital with a variationally determined screening parameter. 


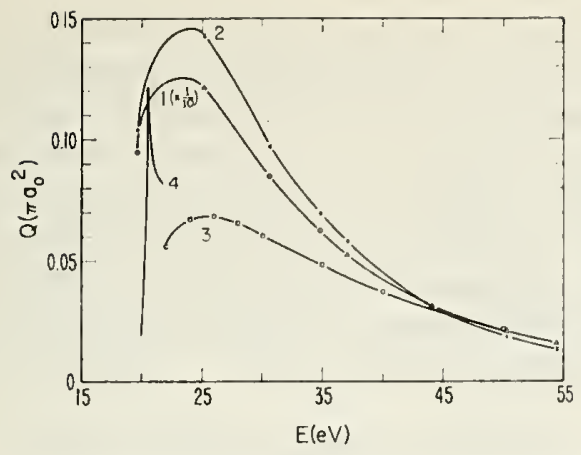

Fig. 31. Total cross sections for the excitation of the $2^{3} S$ state of helium. Curve 1: Born-Oppenheimer approximation (Bell, Eissa, and Moiseiwitsch, 1966). Curve 2: first-order exchange approximation (Bell, Eissa, and Moiseiwitsch, 1966). Curve 3: Ochkur approximation (Ochkur and Bratsev, 1965b). Curve 4: 3-state close coupling approximation (Marriott, 1964).

Even though different ground-state wave functions were employed, the Born-Oppenheimer approximation crosssections calculated by the two groups of investigators were in good agreement. The cross section curves for the $1^{1} S \rightarrow 2^{3} S$ and $1^{1} S \rightarrow 2^{3} P$ excitations calculated using the Born-Oppenheimer approximation are displayed in Figs. 31 and 32 where they are compared with the Ochkur approximation calculations and with more sophisticated calculations which are discussed in subsequent sections.

The values of the Born-Oppenheimer approximation cross sections calculated by Bell, Eissa, and Moiseiwitsch are given in Tables XXVI and XXVII where a comparison is made with the first-order exchange approximation cross sections evaluated by the same authors. We see at once that for electron energies $\lesssim 150 \mathrm{eV}$ the use of the first-order exchange approximation reduces the $1^{1} S \rightarrow 2^{3} S$ excitation cross section given by the Born-Oppenheimer approximation by about a factor of 10 although at higher energies the two approximations tend to each other. Inspection of Table XXVII reveals that for the $1^{1} S \rightarrow 2^{3} P(M=0)$ excitation of helium, the cross section given by the BornOppenheimer approximation is somewhat larger than that given by the first-order exchange approximation for electron energies $\lesssim 50 \mathrm{eV}$ but that above this energy the two approximations yield similar results. For the excitation of the $2^{3} P(M= \pm 1)$ states of helium the two approximations are identical.

Calculations on the excitation of the $2^{3} S$ state of helium using a variety of forms of interaction between the free electron and the atom have been carried out by Joachain and Mittleman (1965) employing the BornOppenheimer approximation and modifications thereof. They used the simple Hylleraas ground-state wave function (122) together with the $2^{3} S$ state wave function given by (131) and hydrogen-like orbitals having variationally determined parameters. The cross sections obtained by them using the post and prior forms of the Born-Oppenheimer approximation are in reasonably satisfactory accordance with each other and with the cross section calculated by Massey and Moiseiwitsch using the Born-Oppenheimer approximation. On the other hand the cross sections calculated by Joachain and Mittleman using post and prior forms of the firstorder exchange approximation are in total disagreement with each other. Moreover their prior interaction cross section is more than a factor of 2 greater than the firstorder exchange approximation cross section calculated by Bell, Eissa, and Moiseiwitsch (1966) who also employed the prior interaction but with a rather better ground-state wave function.

We now come to the interesting calculations carried out by Ochkur (1963) and by Ochkur and Bratsev $(1965$; 1966) based on the so called Ochkur approximation already discussed in Sec. 2.7. An important feature of this approximation is that it cannot give rise to a post-prior discrepancy since it satisfies the principle of detailed balancing. The cross sections calculated by Ochkur (1963) for the $1^{1} S \rightarrow 2^{3} S$ and $1^{1} S \rightarrow 2^{3} P$ excitations, using atomic wave functions having the forms (128) and (129) with the one-electron orbitals being the simple analytical functions given by Veselov, Antonova, Bratsev, and Kirillova (1961), are displayed in Figs. 31 and 32. We see that the Ochkur approximation results in total excitation cross sections which are much smaller than the Born-Oppenheimer approximation cross sections and indeed considerably smaller than the first-order exchange approximation cross sections calculated by Bell, Eissa, and Moiseiwitsch.

Ochkur and Bratsev (1965) have extended the Ochkur approximation calculations to the excitation of many other states of helium, both triplet and singlet, using Hartree-Fock atomic wave functions. Their results are collected together in Tables XXVIII and XXIX. It can be verified from Table XXVIII that the cross sections for the excitation of the triplet states all decay with electron impact energy $E$ according to the law $E^{-3}$ which is much faster than the laws for the excitation of singlet states: $E^{-1}$ for optically forbidden

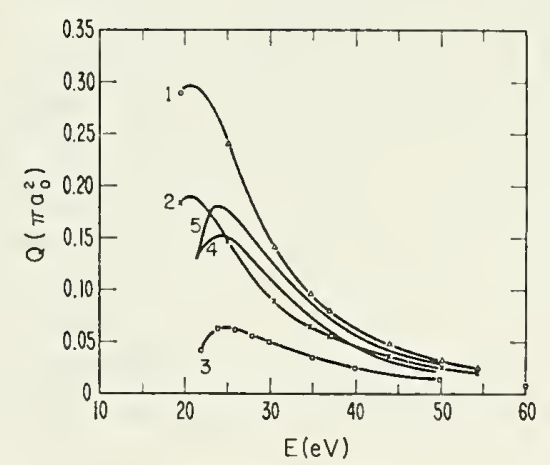

Frg. 32. Total cross sections for the excitation of the $2^{3} P$ states of helium. Curve 1: Born-Oppenheimer approximation (Bell, Eissa, and Moiseiwitsch, 1966). Curve 2: first-order exchange approximation (Bell, Eissa, and Moiseiwitsch, 1966). Curve 3: Ochkur approximation (Ochkur and Bratsev, 1965b). Curve 4: distorted waves approximation including exchange (Massey and Moiseiwitsch, 1960). Curve 5: distorted waves approximation including exchange (Lashmore-Davies, 1965). 
transitions and $E^{-1} \ln E$ for optically allowed transitions. Also the cross sections for the excitation of the states of a given series are proportional to $n^{-3}$, where $n$ is the principal quantum number. Although the validity of the $n^{-3}$ law is justifiable only for large values of $n$ it is found that it holds quite well even for low values of $n$.

Because the cross sections calculated by Ochkur and Bratsev for the excitation of the singlet states of helium include allowance for exchange they should be slightly smaller than the corresponding cross sections resulting from the use of the first Born approximation.

Calculations of the excitation cross sections for the $2^{3} S \rightarrow n^{3} L$ and $2^{3} S \rightarrow n^{1} L$ transitions of helium have also been performed by Ochkur and Bratsev (1966) using the Ochkur approximation together with Hartree-Fock atomic wave functions. The values of their cross sections are displayed in Tables XXX and XXXI. Satisfactory accordance with the earlier calculation of Moiseiwitsch (1957) on the excitation of the $3^{3} P, 3^{3} D$, and $4^{3} D$ states of helium using the Born approximation was found.

\subsection{Distorted Waves Approximation}

The earliest calculations using the distorted waves approximation were performed by Massey and Mohr (1933a) who investigated the $1^{1} S \rightarrow 2^{1} P, 1^{1} S \rightarrow 2^{3} P$ excitations of helium.

More recently Massey and Moiseiwitsch $(1954 ; 1960)$ have calculated cross sections for the excitation of the $2^{1} S, 2^{3} S$, and $2^{3} P$ states of helium using the simple Hylleraas ground-state wave function (122) while Lashmore-Davies (1965) has recalculated the total cross section for the $1^{1} S \rightarrow 2^{3} P$ excitation using the ground state wave function obtained by Green et al. (1954). For the excited states of helium both Massey and Moiseiwitsch $(1954 ; 1960)$ and Lashmore-Davies (1965) used the helium wave functions obtained by Morse, Young, and Haurwitz (1935). Unfortunately their $2^{1} S$ state wave function is not completely orthogonal to the ground-state wave function (122) and so the accuracy of the cross section for the excitation of the $2^{1} S$ state is correspondingly reduced.

The most interesting feature of the distorted waves calculation for the excitation of the $2^{3} S$ state of helium is the appearance of the very sharp peak in the zeroorder partial cross section just above the threshold energy. This peak seems to arise as the consequence of the existence of a doubly excited state $\left(1 s 2 s^{2}\right)^{2} S$ of $\mathrm{He}^{-}$ with energy slightly less than that of the $(1 s 2 s)^{3} S$ metastable state of helium, producing a resonance in the $s$ wave scattering of electrons by the $2^{3} S$ state of helium.

Directing our attention now to the excitation of the $2^{3} P$ states of helium, we see from Fig. 32 that the distorted waves calculations of Massey and Moiseiwitsch (1960) and Lashmore-Davies (1965) are in good agreement with each other although the latter investigator used a rather better ground-state wave function for the helium atom. We also note that allowance for dis- tortion reduces the cross section somewhat below that given by the Born-Oppenheimer approximation (but by not nearly so much as that found in the case of the $1^{1} S \rightarrow 2^{3} S$ excitation) to give cross sections which are not greatly dissimilar from that obtained with the first-order exchange approximation. However we see from Fig. 32 that the cross section curves obtained with the distorted waves approximation both lie well above that given by the Ochkur approximation.

\subsection{Close Coupling Approximation}

Marriott (1964) has carried out an investigation of the scattering of electrons by helium atoms in which he makes full allowance for the coupling between the $1^{1} S, 2^{1} S$, and $2^{3} S$ states. For the $1^{1} S$ ground state he employed the simple Hylleraas wave function given by (122), for the $2^{3} S$ state he used the wave function determined by Morse, Young, and Haurwitz (1935) while for the $2^{1} S$ state he used the wave function derived by Marriott and Seaton (1957) which is orthogonal to the Hylleraas ground-state function (122). The $1^{1} S$ and $2^{3} S$ wave functions are the same as those employed by Massey and Moiseiwitsch (1960) but the $2^{1} S$ state wave function should be superior since the $2^{1} S$ function used by Massey and Moiseiwitsch was not completely orthogonal to the Hylleraas ground-state wave function. The partial and total cross sections for the excitation of the $2^{3} S$ and $2^{1} S$ states of helium obtained by Marriott using the $1^{1} S-2^{1} S-2^{3} S$ close coupling approximation with exchange included are given in Tables XXXII and XXXIII, respectively. The total cross section for the $1^{1} S \rightarrow 2^{3} S$ excitation of helium obtained with the 3 -state close-coupling approximation is also displayed in Fig. 31 where it is compared with the results obtained using less sophisticated methods.

We note that coupling to the $2^{1} P$ and $2^{3} P$ states of helium has been entirely neglected in the above calculations. Because of the strong likelihood that these couplings could have an important effect upon the excitation cross sections, Burke, Cooper, and Ormonde (1966) have carried out a calculation allowing for close coupling to all the five states: $1^{1} S, 2^{1} S, 2^{3} S, 2^{1} P$, $2^{3} P$. They employed Hartree-Fock atomic wave functions. Their cross sections for the excitation of the $2^{1} S, 2^{3} S, 2^{1} P$, and $2^{3} P$ states of helium from the ground state are displayed in Fig. 33.

Marriott $(1957 ; 1966)$ has also investigated the conversion of the $2^{1} S$ state of helium to the $2^{3} S$ state by electron impact:

$$
\mathrm{He}\left(2^{1} S\right)+e \rightarrow \mathrm{He}\left(2^{3} S\right)+e+0.78 \mathrm{eV} .
$$

The cross section for this superelastic collision has been determined experimentally by Phelps (1955) who finds $3 \times 10^{-14} \mathrm{~cm}^{2}\left(340 \pi a_{0}^{2}\right)$ for thermal electrons. However using the $2^{1} S-2^{3} S$ close coupling approximation together with the atomic wave functions for the $2^{1} S$ and $2^{3} S$ states of helium derived by Morse, Young, and 


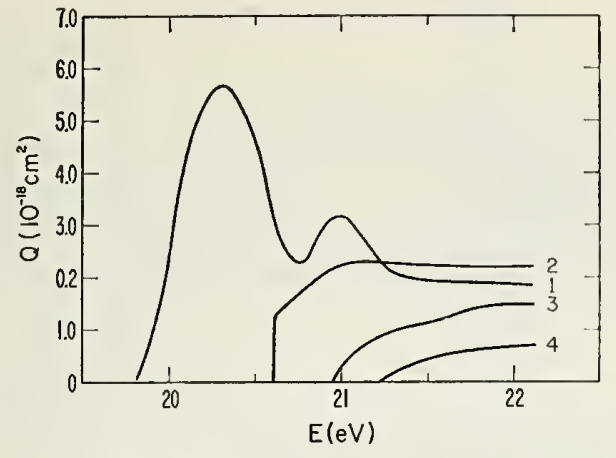

FIG. 33. Total cross sections for the excitation of helium calculated using the five-state close coupling approximation (Burke, Cooper, and Ormonde, 1966). Curve 1: $1^{1} S \rightarrow 2^{3} S$, Curve 2: $1^{1} S \rightarrow 2^{1} S$, Curve $3: 1^{1} S \rightarrow 2^{3} P$, Curve 4: $1^{1} S \rightarrow 2^{1} P$.

Haurwitz (1935), the cross section for the superelastic collision (136) has been calculated by Marriott (1957) to be $60 \pi a_{0}^{2}$ for $0.026-\mathrm{eV}$ electrons which is much smaller than the observed cross section. In a more recent investigation using the $1^{1} S-2^{1} S-2^{3} S-3^{3} S$ close coupling approximation together with the Marriott and Seaton (1957) wave function for the $2^{1} S$ state of helium and a reasonably accurate function for the $3^{3} S$ state, he calculated the cross section for the superelastic collision (136) to be $150 \pi a_{0}^{2}$ at $0.026 \mathrm{eV}$ which is only about a half of that found experimentally by Phelps. This increase in the calculated total cross section at thermal energies is entirely owing to the use of the improved $2^{1} S$ wave function, the effect of including the helium ground state function being small. In Table XXXIV we display the partial and total cross sections for the superelastic conversion of the $2^{1} S$ state to the $2^{3} S$ state of helium calculated using the $1^{1} S-2^{1} S-2^{3} S-$ $3^{3} S$ close-coupling approximation. Marriott (1966) has also calculated the cross section for the $2^{3} S \rightarrow 3^{3} S$ excitation by electron impact for the quartet total spin state using the close coupling approximation.

An investigation of the excitations of the $2^{1} P, 3^{1} P$, and $4^{1} P$ states of helium from the ground state has been carried out by Vainshtein and Dolgov (1959) using the ${ }^{1} S-n^{1} P$ close-coupling approximation with exchange neglected. The importance of the higher-order partial waves, even for energies quite close to the threshold, is easily verified by inspecting Table XXXV where the partial and total cross sections for the $1^{1} S \rightarrow 3^{1} P$ excitation calculated by Vainshtein and Dolgov (1959) are given.

\subsection{Double Excitation of Helium}

The Born approximation has been used by Massey and Mohr (1935) to calculate cross sections for the excitation of the $\left(2 s^{2}\right)^{1} S,(2 s 2 p)^{1} P,(2 s 3 p)^{1} P,(2 s 4 p)^{1} P$, and $(3 s 2 p)^{1} P$ doubly excited states of helium. For the ground state of helium they took the Eckhart function (130) while for the doubly excited states they chose the wave function

$$
\begin{aligned}
\psi_{n_{1} n_{2}}\left(\mathbf{r}_{1}, \mathbf{r}_{2}\right)=N\left\{\phi_{n_{1}}\left(\alpha \mid \mathbf{r}_{1}\right) \phi_{n_{2}}\left(\beta \mid \mathbf{r}_{2}\right)\right. \\
\\
\left.+\phi_{n_{1}}\left(\alpha \mid \mathbf{r}_{2}\right) \phi_{n_{2}}\left(\beta \mid \mathbf{r}_{1}\right)\right\},
\end{aligned}
$$

where $\phi_{n}(\lambda \mid \mathbf{r})$ is a hydrogenic function corresponding to an effective charge $\lambda$. For the $\left(2 s^{2}\right)^{1} S$ and $(2 s 2 p)^{1} P$ states they took $\alpha=2.00, \beta=1.58$; for the $(2 s 3 p)^{1} P$ and $(2 s 4 p)^{1} P$ states they took $\alpha=2.00, \beta=1.00$; while for the $(3 s 2 p)^{1} P$ state they chose $\alpha=1.20, \beta=2.00$. The excitation cross sections calculated by Massey and Mohr (1935) are displayed in Table XXXVI. The smallest cross section occurs for the excitation of the $\left(2 s^{2}\right)^{1} S$ state corresponding to an optically forbidden transition, while the largest cross sections are found for the excitation of the $(2 s 2 p)^{1} P$ and $(3 s 2 p)^{1} P$ states corresponding to optically allowed transitions.

A much more detailed investigation of the excitation of doubly excited states of helium has been carried out by Becker and Dahler (1964). They began their study by using the Born-Oppenheimer approximation to calculate the cross sections for the excitation of the $\left(2 p^{2}\right)^{3} P,(2 p 3 p)^{1} P,(2 p 3 d)^{1} D$, and $(2 p 3 d)^{3} D$ states of helium, none of which are subject to autoionization. For the ground state they used the simple Hylleraas function (122) and the Eckhart function (130) while for the doubly excited states they employed symmetrized wave functions composed of products of hydrogenic orbitals. Their cross section curves are given in Fig. 34. By far the largest cross section arises from the excitation of the $\left(2 p^{2}\right)^{3} P$ state, the lowest of the doubly excited states which is stable towards autoionization. Becker and Dahler also used the distorted waves method and the two state close coupling approximation,

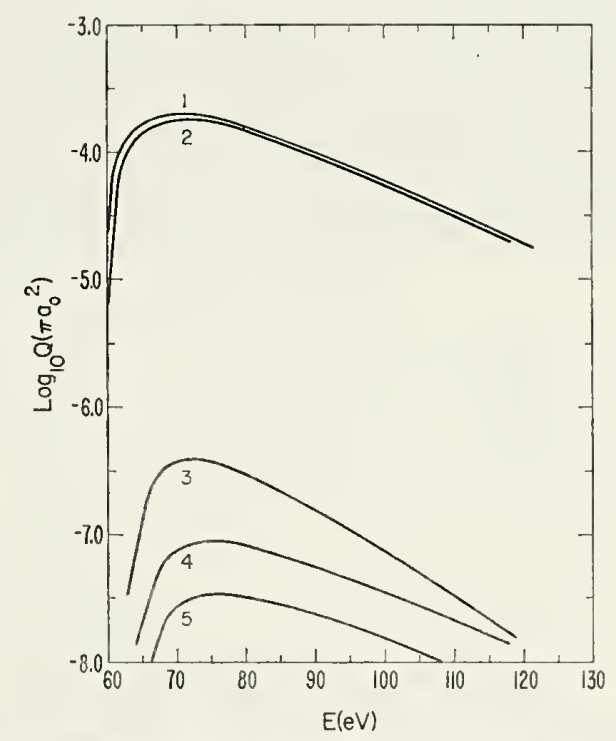

FIg. 34. Cross sections for the excitation of doubly excited states of helium calculated using the Born-Oppenheimer approximation (Becker and Dahler, 1964). Curve 1: $\left(2 p^{2}\right)^{3} P$ excitation using Hylleraas ground-state function. Curve 2: $\left(2 p^{2}\right)^{3} P$ excitation using Eckhart ground-state function. Curve 3: $(2 p 3 p)^{1} p$ excitation. Curve 4: $(2 p 3 d)^{1} D$ excitation. Curve $5:(2 p 3 d)^{3} D$ excitation. 
both with exchange included, to investigate in further detail the excitation of the $\left(2 p^{2}\right)^{3} P$ state of helium, the ground state being represented by the simple Hylleraas wave function (122). The resulting cross sections are displayed in Fig. 35. We see that the distorted waves method and the two state close coupling approximation give rise to practically indistinguishable cross section curves. The implication is that the coupling between the ground state and the doubly excited state is fairly weak, a conclusion which is supported by the smallness $\left(\sim 10^{-20} \mathrm{~cm}^{2}\right)$ of the excitation cross section.

\section{Neon and Argon}

Extensive calculations using the Born approximation have been carried out by Veldre, Lyash, and Rabik $(1965 \mathrm{a} ; 1965 \mathrm{~b})$ on the excitation of neon and argon by electron impact.

We first consider the case of neon excitation. Because of the lack of Hartree-Fock wave functions, hydrogenic orbitals were used for the different electron shells in the neon atom. Total excitation cross sections were calculated for transitions between all the terms of the configurations $(\gamma) 2 p,(\gamma) 3 s,(\gamma) 3 p,(\gamma) 4 s,(\gamma) 3 d,(\gamma) 4 p$, and $(\gamma) 5 s$, where $\gamma$ signifies the core configuration $1 s^{2} 2 s^{2} 2 p^{5}$. Because the formulation was based upon the assumption that $L S$ coupling prevails for all configurations of the neon atom, and this is known to be invalid for excited inert gas atoms, the total excitation cross sections calculated by Veldre, Lyash, and Rabik (1965b) could be seriously in error.

$L S$ coupling holds for the ground ${ }^{1} S_{0}$ state of an inert gas atom; however for moderately excited states of inert gas atoms $j l$ coupling holds while for highly excited state $j j$ coupling occurs. Realizing this, Veldre, Lyash, and Rabik (1965a) investigated the consequences of choosing different types of coupling ( $L S, j l$, $j j$ ) between the optical electron and the core of the excited atom. The wave function of the optical electron was calculated by solving the radial equation for a particle in the field produced by Gaspar's potential

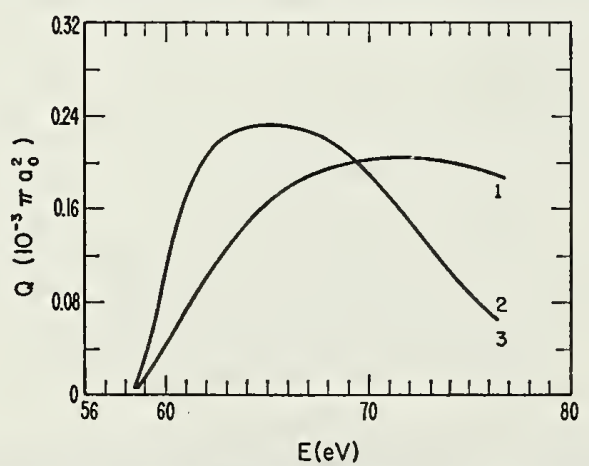

Fig. 35. Cross sections for the excitation of the doubly excited $\left(2 p^{2}\right)^{3} P$ state of helium (Becker and Dahler, 1964). Curve 1: Born-Oppenheimer approximation. Curve 2: distorted waves approximation. Curve $3: 2$ state close coupling approximation (Curve 3 is identical to Curve 2).

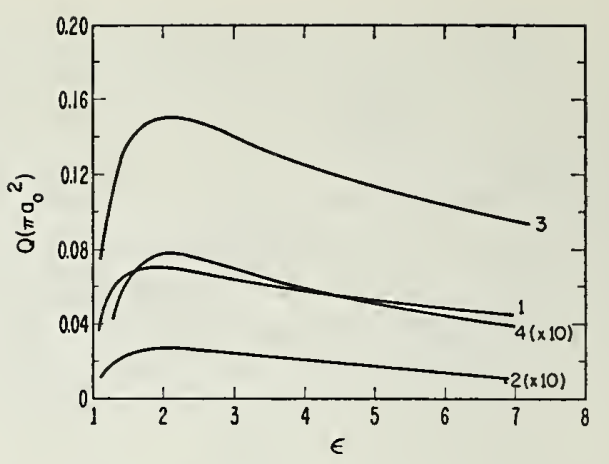

Fig. 36. Total cross sections for the excitations of neon and argon from their ground ${ }^{1} S_{0}$ states calculated using the first Born approximation. [ $\epsilon$ is electron energy in threshold units defined by Eq. (38).] Curve 1: $1 s^{2} 2 s^{2} 2 p^{5} 3 p^{1} S_{0}$ excitation of neon using $L S$ coupling for the ground and excited states. When $j l$ coupling is used for the excited state, cross section is reduced by factor of 3 . Curve 2: $1 s^{2} 2 s^{2} 2 p^{5} 3 p^{1} D_{2}$ excitation of neon using $L S$ coupling for the ground and excited states. When $j l$ coupling is used for the excited state, cross section is reduced by factor of 15 . Curve 3 : $1 s^{2} 2 s^{2} 2 p^{6} 3 s^{2} 3 p^{5} 4 p^{1} S_{0}$ excitation of argon using $L S$ coupling for the ground and excited states. When $j l$ coupling is used for the excited state, cross section is reduced by factor of 3 . Curve 4 : $1 s^{2} 2 s^{2} 2 p^{6} 3 s^{2} 3 p^{5} 4 p^{1} D_{2}$ excitation of argon using LS coupling for the ground and excited states. When $j l$ coupling is used for the excited state, cross section is reduced by factor of 15 .

(1952), which has an analytical form and yields good wave functions for atoms with fairly large nuclear charge such as neon and argon. They derived wave functions for the neon configurations $(\gamma) 2 p,(\gamma) 3 s$, $(\gamma) 3 p,(\gamma) 4 s,(\gamma) 3 d,(\gamma) 4 p$, and $(\gamma) 5 s$, where $\gamma$ denotes the core configuration $1 s^{2} 2 s^{2} 2 p^{5}$, and for the argon configurations $\left(\gamma^{\prime}\right) 3 p, \quad\left(\gamma^{\prime}\right) 4 s, \quad\left(\gamma^{\prime}\right) 4 p, \quad\left(\gamma^{\prime}\right) 3 d, \quad\left(\gamma^{\prime}\right) 5 s$, $\left(\gamma^{\prime}\right) 5 p$, and $\left(\gamma^{\prime}\right) 6 s$, where $\gamma^{\prime}$ signifies the core configuration $1 s^{2} 2 s^{2} 2 p^{6} 3 s^{2} 3 p^{5}$.

For the excitation from the ground state to an excited state, $L S$ coupling was used to characterize the ground state while $L S, j l$, and $j j$ couplings were chosen for the excited state of the inert gas atom. Their results show that the largest cross section is obtained when $L S$ coupling is chosen for the excited state while the least cross section occurs with $j l$ coupling. This can be seen from Fig. 36 where the total cross sections for the excitation of the states of the configuration $(\gamma) 3 p$ from the ground ${ }^{1} S_{0}$ state of neon and the excitation of the states of the configuration $\left(\gamma^{\prime}\right) 4 p$ from the ground ${ }^{1} S_{0}$ state of argon, using $L S$ and $j l$ coupling for the excited states, are compared.

In the case of transitions between excited states of the inert gas atom, the same type of coupling between the optical electron and the core of the atom was used for both states, either $L S$ or $j l$ coupling.

As a consequence of the fact that a change in the type of coupling between the initial and final states. of the inert gas atom produces a considerable reduction in the total excitation cross section, Veldre, Lyash, and Rabik (1965a) found that the cross sections for the excitation of ground-state inert gas atoms have much 
smaller values than the cross sections for the excitation of excited atoms.

Calculations of the excitation cross sections for $(\gamma) 2 p \rightarrow(\gamma) 3 p$ transitions of neon have also been carried out by Boikova and Fradkin (1965) using the Ochkur approximation together with $L S, j l$, and $j j$ coupling representations of the $(\gamma) 3 p$ excited states of the neon atom.

\section{Mercury}

The excitation of mercury to the $6^{3} P_{0}, 6^{3} P_{1}, 6^{3} P_{2}$, and $6^{1} P_{1}$ states from the ground $6^{1} S_{0}$ state has been investigated by Penney (1932a) using the Born-Oppenheimer approximation. He assumed that the wave function of the core remained unaltered during the transition and used the Coulomb approximation to determine the radial wave functions of the two electrons outside the core. The total wave functions of the $S$ and $P$ states of mercury were then expressed according to the formulac derived by Houston (1929). Penney found that his wave functions yielded oscillator strengths for the $6^{1} P_{1} \rightarrow 6^{1} S_{0}$ and $6^{3} P_{1} \rightarrow 6^{1} S_{0}$ transitions which were about twice the experimentally determined values. $\mathrm{He}$ therefore only gave relative cross sections for the excitation of the $P$ states of mercury.

The calculations are not very accurate. Thus it should be noted that the thresholds for the excitations of the ${ }^{3} P_{0},{ }^{3} P_{1}$, and ${ }^{3} P_{2}$ states are not in the order of increasing energy as they should be. However, the calculations clearly indicate that the cross section for the excitation of the $6^{1} P_{1}$ state is considerably larger than those for the excitation of the ${ }^{3} P$. states, particularly for electron impact energies above about $15 \mathrm{eV}$, and that it has a broad maximum located some distance from the threshold energy whereas the cross sections for the excitation of the ${ }^{3} P$ states are more sharply peaked and positioned close to the threshold.

The above calculations have been repeated by Yavorskii (1947) employing precisely the same method and wave functions. His excitation cross sections, which were given in absolute units, are not in satisfactory accordance with the relative cross sections calculated by Penney. However, the threshold energies obtained by Yavorskii are in the correct order. Regrettably the values $3.71 \times 10^{-16} \mathrm{~cm}^{2}$ and $1.05 \times 10^{-16} \mathrm{~cm}^{2}$ for the $6^{1} S_{0} \rightarrow 6^{1} P_{1}$ and $6^{1} S_{0} \rightarrow 6^{3} P_{1}$ excitation cross sections at their maxima, quoted by Yavorskii in the text of his paper, are somewhat greater than the values of the maximum cross sections obtainable from his figure.

Recently more reliable calculations of the excitation cross sections for the $6^{3} P_{0}, 6^{3} P_{1}, 6^{3} P_{2}$, and $6^{1} P_{1}$ states of mercury, employing the Ochkur approximation together with Coulomb approximation functions yielding oscillator strengths for the $6^{1} P_{1} \rightarrow 6^{1} S_{0}$ and $6^{3} P_{1} \rightarrow 6^{1} S_{0}$ transitions in good agreement with the experimentally determined values, have been performed by McConnell and Moiseiwitsch (1968). Their cross sections are displayed in Fig. 37.

\section{Alkali Metal Atoms}

We devote this section to the consideration of the alkali metal atoms each of which is composed of a single electron outside a core of completely filled electro. shells. In the ground state of an alkali metal atom the outer electron is in an $n s$ state with $n=2,3,4,5,6$ for lithium, sodium, potassium, rubidium, and cesium, respectively. We are mainly concerned with $n s \rightarrow n p$ excitations of the outer electron of the alkali metal atoms.

Because of the very strong coupling between the initial and final states of the resonance transition $n s \rightarrow n p$, the Born approximation produces excitation cross sections which are too large close to the threshold energy. Born approximation calculations have been carried out by Bates et al. (1950) and by Vainshtein (1965) on the $3 s \rightarrow 3 p$ transition of sodium, and by Vainshtein, Opykhtin, and Presnyakov (1964b) on the resonance transitions of lithium, sodium, potassium, rubidium, and cesium whose excitation cross sections are presented in Table XXXVII. We note the very large values of these Born approximation cross sections at their maxima. Vainshtein has also applied the distorted waves approximation with exchange neglected to the $3 s \rightarrow 3 p$ transition of sodium finding that allowance for distortion introduces an oscillation into the excitation cross section with several maxima. Some of the partial cross sections obtained by Vainshtein exceed the theoretical upper bound imposed by the unitary property of the $\mathbf{S}$ matrix, clearly demonstrating the strength of the coupling.

To overcome the difficulties arising from the very strong coupling between the $3 s$ and $3 p$ states of sodium, Seaton (1955a) has used his modification of the Bethe approximation, previously discussed in section 2.9, to calculate the total excitation cross section for the $3 s \rightarrow 3 p$ resonance transition of sodium. The cross section obtained by Seaton is displayed in Fig. 38. We see from this figure that the modified Bethe approximation produces a considerable reduction of the excitation cross section near the threshold energy.

Calculations on the $3 s \rightarrow 3 p$ resonance transition of sodium using the semi-classical impact parameter method have also been carried out by Seaton (1962). He obtains an excitation cross section which is much below that given by the Born approximation at low impact energies, in accordance with the modified Bethe approximation.

Salmona and Seaton (1961) have used the unitarized Born approximation, discussed earlier in Sec. 2.14, to calculate the total excitation cross section for the $3 s \rightarrow 3 p$ transition of sodium. In this method the Born approximation to the reactance matrix $\mathbf{R}$ is substituted into the formula for the transmission matrix, thus ensuring that the unitary property of the scattering matrix is satisfied. A further approximation made by Salmona and Seaton was the neglect of all short range interac- 
tions, just the long range interaction $S / r^{2}$ being retained. The line strength $S$ has been determined by $f$ value measurements to be 19 atomic units which again emphasizes the great strength of the coupling between the $3 s$ and $3 p$ states of sodium. The neglect of the short range interactions is valid for large values of the angular momenta of the incident electron while the use of an approximation which satisfies the conservation theorem ensures that the small angular momenta are treated without undue error. Inspection of Fig. 38 shows that the method used by Salmona and Seaton (1961) provides a cross section curve which is in satisfactory accordance with the modified Bethe approximation.

More sophisticated calculations which take account of the very strong coupling between the $3 s$ and $3 p$ states of sodium although neglecting exchange have been carried out by Lane and Lin (1964) and by Barnes, Lane, and Lin (1965). Designating the total angular momentum quantum number by $L$ and the angular momentum quantum numbers of the atomic and free electrons by $l_{1}$ and $l_{2}$, respectively, the following three states of the total system enter into the excitation problem if we neglect coupling to all states of the sodium atom other than the initial state $3 s$ and the final state $3 p$ :

$\begin{array}{cccc}\nu & l_{1} & \ell_{2} & L \\ 1 & 0 & \ell & \ell \\ 2 & 1 & \ell-1 & \ell \\ 3 & 1 & \ell+1 & \ell\end{array}$

There is also a fourth state of the total system with quantum numbers $l_{1}=1, l_{2}=\ell, L=\ell$ but this has a different parity to the other three states and so need not be considered here. For each value of $\ell$ we have therefore three coupled differential equations to solve. Because the energies of the $3 s$ and $3 p$ states of sodium are only $2.10 \mathrm{eV}$ apart, Lane and Lin (1964) made the reasonable approximation that the initial and final states are in exact resonance thus enabling them to decouple the differential equations. In addition, following Salmona and Seaton (1961), they neglected all the short-range interactions retaining only the long-

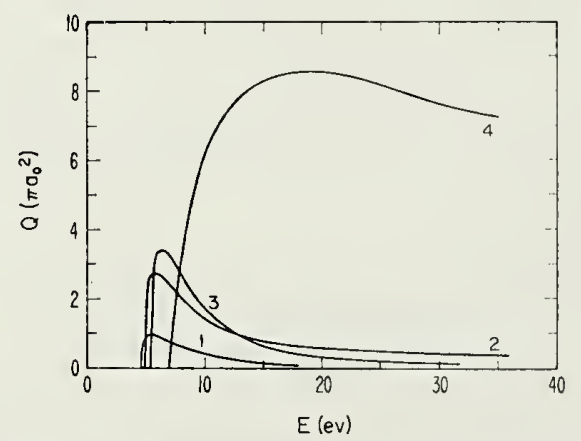

FIG. 37. Total cross sections for the excitation of mercury calculated using the Ochkur approximation (McConnell and Moiseiwitsch, 1968). Curve 1: $6^{1} S_{0} \rightarrow 6^{3} P_{0}$ excitation. Curve 2: $6^{1} S_{0} \rightarrow$ $6^{3} P_{1}$ excitation. Curve 3: $6^{1} S_{0} \rightarrow 6^{3} P_{0}$ excitation. Curve 4: $6^{1} S_{0} \rightarrow$ ${ }^{1} P_{1}$ excitation.

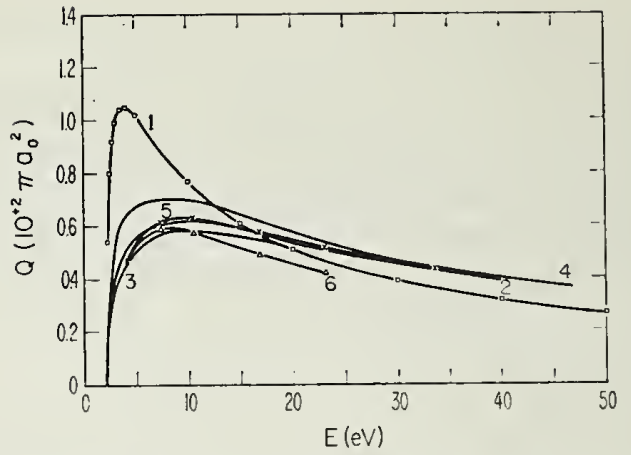

Frg. 38. Total cross sections for the $3 s \rightarrow 3 p$ excitation of sodium. Curve 1: first Born approximation (Karule and Peterkop, 1965). Curve 2: modified Bethe approximation (Seaton, 1955). Curve 3: impact parameter method (Seaton, 1962). Curve 4: unitarized Born approximation (Salmona and Seaton, 1961). Curve 5: resonance distortion method (Lane and Lin, 1964). Curve 6: $3 s-3 p$ close coupling approximation neglecting exchange (Barnes, Lane, and Lin, 1965).

range interaction $S / r^{2}$. Then employing the exact resonance solution corresponding to the incident channel $\nu=1$ they performed a single iteration to obtain more accurate solutions of the other two differential equations, the exact resonance approximation being discarded. All the partial waves from $\ell=2$ to 15 were treated in this manner. However, because of the great strength of the $S / r^{2}$ interaction, they were unable to carry out the same procedure for the $\ell=0$ and 1 waves and so arbitrarily chose their partial cross sections to be $\frac{1}{2} \pi(2 \ell+1) / k_{1}{ }^{2}$ which is half the theoretical maximum imposed by the conservation theorem. For the partial cross sections associated with $\ell>15$ they used the Bethe approximation. The resulting total cross section curve is displayed in Fig. 38. We see that it is in close accordance with the cross section obtained by Seaton using the modified Bethe approximation.

Barnes, Lane, and Lin (1965) have solved the three coupled differential equations corresponding to the states $\nu=1,2,3$ of the total system by numerical integration. They included the short-range interactions in this investigation using hydrogenic wave functions for the optical electron with an effective charge of 2.92 and performed the calculation for all values of $\ell$ up to 15. The partial cross sections obtained by them together with their total cross sections, the contribution from the partial waves with $\ell>15$ being estimated with the Bethe approximation, are displayed in Tables XXXVIII and XXXIX. We also give their total cross section curve in Fig. 38. It is somewhat lower than the cross section curves obtained with the less accurate methods discussed above.

Close coupling calculations on the resonance transitions $n s \rightarrow n p$ of lithium, sodium, potassium and cesium have been carried out by Karule and Peterkop (1964; 1965) for electron impact energies close to the threshold. The first calculations performed by Karule and Peterkop (1964) were concerned with the $2 s \rightarrow 2 p$ excitation of lithium and were carried out at energies of 2.0, 2.5 
and $3.0 \mathrm{eV}$ both including and neglecting exchange. They used the $1 s$ and $2 s$ analytical wave functions derived by Clementi, Roothaan and Yoshimine (1962) and a hydrogenic $2 p$ wave function. Karule and Peterkop (1965) subsequently repeated the lithium calculations using Hartree-Fock wave functions (without allowance for the effect of the outer electron on the core) (Ivanova and Ivanova, 1964) but found that the changes in the partial cross sections were slight. In addition they extended their investigation to sodium, potassium, and cesium using semi-empirical atomic wave functions for the outer electron. Their calculations were carried out for the $L=0,1, \cdots, 8$ partial waves neglecting exchange, for the $L=0,1,2,3$ partial waves including exchange for sodium and potassium, and for the $L=0,1,2,3,4$ partial waves including exchange for cesium. The agreement with the calculations of Barnes, Lane, and Lin (1965) for sodium with exchange neglected is satisfactory at $4 \mathrm{eV}$, apart from the $L=0$ and 1 partial waves. The lack of accordance for these low order partial waves is presumably the consequence of the use of different atomic sodium wave functions.

Karule and Peterkop (1965) also calculated the partial sum

$$
\sum_{L=0}^{8} Q^{L}
$$

by adding the partial cross sections for the low $L$ values, obtained including exchange, to the partial cross sections for the higher $L$ values, obtained neglecting exchange. Since the partial cross sections corresponding to $L>8$ make a significant contribution to the total cross section, this means that their values of the partial sum

$$
\sum_{L=0}^{8} Q^{L}
$$

are considerably lower than the values of the total cross section. This can be exemplified by considering the $6 s \rightarrow 6 p$ transition of cesium for which Hansen (1964) has carried out a modified Bethe approximation calculation. His total excitation cross sections are displayed in Table XL. We see that at $5 \mathrm{eV}$ his total $6 s \rightarrow 6 p$ excitation cross section is $114 \pi a_{0}^{2}$ whereas the partial sum

$$
\sum_{L=0}^{8} Q^{L}
$$

obtained by Karule and Peterkop is only $73 \pi a_{0}^{2}$.

Finally we come to the cross sections for other excitations of the alkali metal atoms. Born approximation calculations on the $3 s \rightarrow 3 d$ and $3 s \rightarrow 4 p$ excitations of sodium have been carried out by Bates et al. (1950) using Hartree-Fock wave functions; on the $3 s \rightarrow 4 s$, $3 s \rightarrow 4 p, 4 s \rightarrow 4 p, 5 s \rightarrow 6 p$, and $5 s \rightarrow 7 p$ excitations of sodium by Vainshtein (1965) using semi-empirical wave functions for the optical electron; and on an im- mense number of transitions in lithium, sodium, potassium, rubidium, and cesium by Vainshtein, Opykhtin, and Presnyakov (1964a) also using semi-empirical wave functions for the outer electron. The Born approximation excitation cross sections obtained by Vainshtein (1965) and by Vainshtein, Opykhtin, and Presnyakov (1964a) are displayed in Tables XLI-XLV.

\section{Oxygen and Nitrogen Atoms}

In the present section we are mainly concerned with the excitation of the ${ }^{1} D$ and ${ }^{1} S$ terms of the lowest configuration $1 s^{2} 2 s^{2} 2 p^{4}$ of atomic oxygen from the ground ${ }^{3} P$ term of this configuration.

The first calculations of the cross sections for these excitations were carried out by Yamanouchi, Inui, and Amemiya (1940) who employed a simplified version of the distorted waves approximation. They found that their ${ }^{3} P \rightarrow{ }^{1} D$ and ${ }^{3} P \rightarrow{ }^{1} S$ excitation cross sections were dominated entirely by the $p$ wave contribution arising from the incident electron. This being so Bates et al. (1950) were able to establish that at their maxima these excitation cross sections were factors of 89 and 66 times greater, respectively, than the upper bound imposed by the conservation theorem.

As a consequence of the above situation, Seaton (1953a) was led to make a detailed investigation of the oxygen excitation problem for the incident $p$ wave ${ }^{2} P$ scattering case. He showed that the assumption of weak coupling, inherent in the distorted waves approximation, was quite invalid for the excitation problem under consideration. Seaton therefore decided upon an entirely different approach based upon the fact that the excitation energies of the ${ }^{1} D$ and ${ }^{1} S$ terms of the lowest

\begin{tabular}{|c|c|c|c|c|}
\hline \multirow[b]{2}{*}{ Term } & \multirow[b]{2}{*}{$n$} & \multirow{2}{*}{$\begin{array}{c}\text { Statistical } \\
\text { weight } \\
\omega_{n}\end{array}$} & \multicolumn{2}{|c|}{$\begin{array}{l}\text { Excitation energy } \\
\text { (rydbergs) }\end{array}$} \\
\hline & & & Observed & $\begin{array}{l}\text { Hartree } \\
\text { Fock }\end{array}$ \\
\hline${ }^{3} P$ & 1 & 9 & 0 & 0 \\
\hline${ }^{1} D$ & 2 & 5 & 0.145 & 0.152 \\
\hline${ }^{1} S$ & 3 & 1 & 0.308 & 0.378 \\
\hline
\end{tabular}
configuration of oxygen are rather small, as can be readily verified from the following table:

On expanding the Coulomb interactions $e^{2} / r_{i j}$ between the electrons of the oxygen atom using the multipole expansion formula (89) it is found that the energies of the ${ }^{3} P,{ }^{1} D$, and ${ }^{1} S$ states of the lowest configuration depend only upon the $\lambda=0,1,2$ terms of the expansion, the main contribution arising from the spherically symmetrical terms associated with $\lambda=0$. The neglect of the terms corresponding to $\lambda=2$ produces equal energies for the ${ }^{3} P,{ }^{1} D$, and ${ }^{1} S$ states and so 
the approximation in which the $\lambda=2$ terms are omitted is referred to as the exact-resonance approximation. There are also exchange terms between the $2 p$ electrons and the $1 s$ and $2 s$ electrons belonging to the core of the oxygen atom which correspond to $\lambda=1$. When the $\lambda=2$ terms are omitted, it is found that these $\lambda=1$ exchange terms do not give rise to energy differences.

With the above consideration in mind, Seaton (1953a) introduced the following approximations in dealing with the oxygen excitation problem which together compose his approximation I:

(i) the energy differences between the ${ }^{3} P,{ }^{1} D$, and ${ }^{1} S$ states of the lowest configuration can be neglected,

(ii) the $2 p$ orbitals of the three states of the lowest configuration can be taken to be the same and equal to the Hartree-Fock function for the ${ }^{1} D$ state,

(iii) all the $\lambda=2$ terms in the integrodifferential equations describing the scattering can be neglected,

(iv) all the $\lambda=1$ terms corresponding to exchange between the incident $p$ wave electron and the $1 s$ and $2 s$ core electrons can be neglected.

Approximation I enables the three integrodifferential equations describing the excitation problem for the $p$ wave ${ }^{2} P$ scattering case to be uncoupled and an exact solution to the equations derived. Using this solution as a first approximation, Seaton then discarded approximation (i) and obtained a better approximation to the solution of the three original coupled integrodifferential equations by carrying out a single iteration. This he called approximation II.

To test the accuracy of approximations I and II, Seaton proceeded to carry out more detailed calculations at a single electron impact energy corresponding to the value of the wave number $k_{1}$ of the incident electron given by $k_{1}{ }^{2}=0.3$. This energy was deliberately chosen to be insufficiently high to excite the ${ }^{1} S$ term of the ground configuration, so that only two coupled equations are involved if the polarization resulting from the presence of the ${ }^{1} S$ term is neglected. He first carried out the same procedure as for approximation II but discarding approximations (iii) and (iv). This Seaton refers to as approximation III. Finally he solved the original pair of coupled equations exactly to give him his approximation IV. The values of the collision strengths obtained by Seaton for $k_{1}{ }^{2}=0.3$ are displayed in Table XLVI, the numerals, 1, 2, 3 signifying the ${ }^{3} P$, ${ }^{1} D,{ }^{1} S$ terms, respectively. We see that exchange between the incident $p$ electron and the core $1 s$ and $2 s$ electrons is fairly important and that approximation III, which allows for this exchange, provides collision strengths which are in rather good agreement with the values given by the exact treatment of the coupled equations. However we also note that $\Omega^{p}(1,2)$ and $\Omega^{p}(2,1)$ differ by about $16 \%$ in approximation IV whereas detailed balancing requires them to be equal. This is just the so called post-prior discrepancy and is an indication of the accuracy of the wave functions
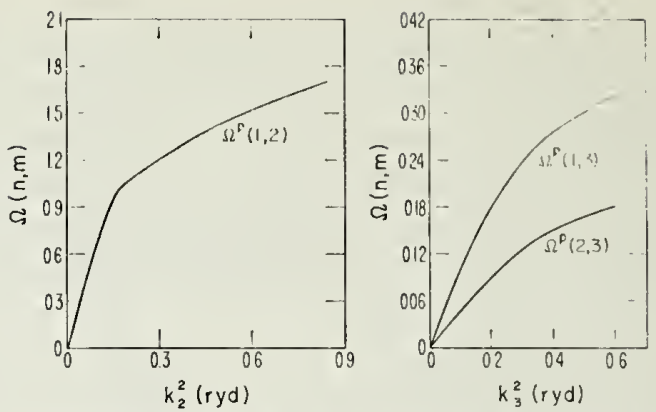

FIG. 39. Collision strengths $\Omega^{p}(1,2), \Omega^{p}(1,3)$, and $\Omega^{p}(2,3)$ for transitions connecting the ${ }^{3} P,{ }^{1} D,{ }^{1} S$ terms of the lowest configuration of atomic oxygen (Seaton, 1953a).

which were used for the oxygen atom. Since it was not possible to decide which of $\Omega^{p}(1,2)$ and $\Omega^{p}(2,1)$ provide the most reliable values for the collision strength, Seaton chose the geometric mean given by $\bar{\Omega}(1,2)=$ $[\Omega(1,2) \Omega(2,1)]^{1 / 2}$.

With regard to the $p$ wave ${ }^{2} D$ scattering case, Seaton used the distorted waves method to calculate the collision strength $\Omega^{p}(1,2)$ at $k_{1}{ }^{2}=0.3$. He obtained the value 0.007 which is less than $1 \%$ of the corresponding value for the $p$ wave ${ }^{2} P$ scattering case. In addition the $d$ wave contribution to the collision strength $\Omega^{d}(1,2)$ was found by Yamanouchi, Inui, and Amemiya (1940) to increase slowly from zero at the threshold to the value 0.077 at $k_{1}{ }^{2}=1$ and to a maximum value of 0.95 at $k_{1}^{2}=4$; thus we see that $\Omega^{d}(1,2)$ is unimportant within the range of electron impact energies concerning us here.

The final collision strengths $\Omega^{p}(1,2), \Omega^{p}(1,3)$, and $\Omega^{p}(2,3)$ obtained by Seaton in his first paper (1953a) are displayed in Fig. 39. They were derived by multiplying the approximation II values of $\Omega^{p}(1,2), \Omega^{p}(1,3)$, and $\Omega^{p}(2,3)$ by the factor 1.31 which yields agreement with the approximation III value at $k_{1}^{2}=0.3$. A satisfactory feature of these values is that they satisfy the conservation theorem

$$
\Omega^{\ell}\left(n, n^{\prime}\right) \leq(2 \ell+1) \omega_{<},
$$

where $\omega_{<}$is the lesser of the statistical weights $\omega_{n}, \omega_{n^{\prime}}$ of the states $n, n^{\prime}$. We note that a discontinuity in the slope of $\Omega^{p}(1,2)$ occurs at the threshold energy for the excitation of the ${ }^{1} S$ state owing to the neglect of this state below its excitation threshold.

In a subsequent paper Seaton (1955a) further investigated the accuracy of his calculations on the $\Omega^{p}(1,3)$ and $\Omega^{p}(2,3)$ collision strengths and introduced minor alterations. He also calculated the $\Omega^{d}(2,3)$ collision strength finding that it was not unimportant. The final values of his collision strengths (Seaton, 1956) are displayed in Table XLVII, the corresponding cross sections being given by the formulas

$$
\Omega\left(n, n^{\prime}\right)=\left(\omega_{n} k_{n}^{2} / \pi\right) Q_{n, n^{\prime}}=\left(\omega_{n^{\prime}} k_{n^{\prime}}{ }^{2} / \pi\right) Q_{n^{\prime}, n} .
$$

The appropriate statistical weights are to be found in (139). It has been estimated by Seaton that his calcu- 


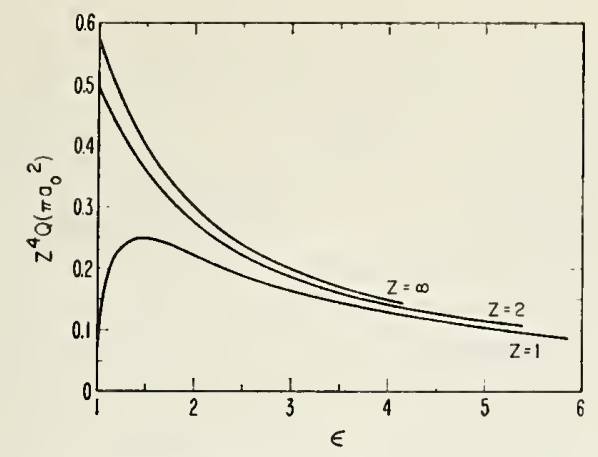

Fig. 40. Total cross sections for the $1 s \rightarrow 2 s$ excitation of hydrogenic ions of charge $Z$ calculated using the Coulomb-Born approximation (first Born approximation for $Z=1$ ) (Tully, 1960). [ $\epsilon$ is electron energy in threshold units defined by Eq. (38).]

lated collision strengths are accurate to within about $40 \%$ while their relative values should be still more reliable. The extrapolated values given in italics in Table XLVII are probably accurate to within about a factor of 2 .

A prescription for obtaining the collision strengths for transitions between the ${ }^{4} S,{ }^{2} D,{ }^{2} P$ terms of the lowest configuration $1 s^{2} 2 s^{2} 2 p^{3}$ of atomic nitrogen has been given by Seaton (1956). Denoting these terms by $1,2,3$ respectively, the collision strengths $\Omega(1,2)$, $\Omega(1,3)$, and $\Omega(2,3)$ can be obtained from Table XLVII by multiplying the corresponding oxygen collision strengths by $25 / 24,25 / 8$, and $225 / 16$, respectively.

More accurate calculations on the excitation of atoms having a ground state $2 p^{q}$ configuration involving the solution of coupled equations are in progress and a paper describing the formulation has been published by Smith, Henry, and Burke (1966).

The probability per unit time for an atom in a state $n$ to make a $n \rightarrow n^{\prime}$ transition as the result of electron impact can be expressed in the form $\alpha_{n n^{\prime}} n(e)$, where $n(e)$ is the number density of electrons. Assuming a Maxwellian velocity distribution of electrons, Seaton $(1955 ; 1956)$ has calculated the deactivation coefficients $\alpha_{21}, \alpha_{31}$, and $\alpha_{32}$ for atomic oxygen and atomic nitrogen. These are displayed in Table XLVIII.

Finally we note that $p$ wave collision strengths for the excitation of the $2 p^{3}\left({ }^{4} S\right) 3 p^{3} P$ and ${ }^{5} P$ states of oxygen from the ground $2 p^{4}{ }^{3} P$ state have been calculated by Percival (1957) using the distorted waves method.

\section{Positive Ions}

The excitation of positive ions is dominated by the Coulomb field which acts upon the incident electron throughout its trajectory and indeed distorts it from a linear path even at infinite separation. The effect of the Coulomb field is so powerful that the threshold behavior of the excitation cross section $Q$ is entirely different to that exhibited by the neutral atom case. This is best illustrated by the hydrogen-like ions towards which we now direct our attention.

\subsection{Hydrogenic Ions}

We consider an ion having nuclear charge $Z$ and possessing a single electron only. The $1 s \rightarrow 2 s, 1 s \rightarrow 3 s$, and $1 s \rightarrow 2 p$ excitations of such ions have been examined by Tully (1960), Burgess (1961), and Gailitis (1963) using the Coulomb-Born approximation. This is analogous to the customary Born approximation with, however, Coulomb waves representing the incident and scattered electrons instead of plane waves. Because the Coulomb field exerts such a strong force upon the incident electron, the Coulomb-Born approximation is more accurate for scattering by positive ions than the Born approximation is for scattering by neutral atoms. In Figs. 40 and 41 we display $Z^{4} Q$ plotted against the energy of the incident electron in threshold units for the $1 s \rightarrow 2 s$ and $1 s \rightarrow 2 p$ transitions calculated by Tully (1960) and by Burgess (1961), respectively. We see immediately that for positive ions the total excitation cross section monotonically increases to a nonzero value at threshold whereas for neutral atoms the Born approximation yields a cross section which vanishes at threshold.

A useful formula which is sometimes employed to estimate excitation cross sections for optically allowed transitions, especially for the case of positive ions, is based upon the replacement of the potential $V_{n 1}$ by its asymptotic form (90) at large separations. Introducing the oscillator strength $f(n, 1)$ given by (92), we find that the total excitation cross section can be written

$$
Q_{n}=\frac{k_{n}}{k_{1}} \frac{a_{0}^{2} f(n, 1)}{\Delta E_{n 1}} \int\left|\int F_{n} \frac{\mathbf{r}}{r^{3}} F_{1} d \mathbf{r}\right|^{2} d \omega,
$$

where $F_{1}$ and $F_{n}$ are the wave functions describing the incident and scattered electrons, $\Delta E_{n 1}$ is the excitation energy in rydberg units, and $d \omega$ is an element of solid angle in the direction of scattering.

If we now make use of the free-free Kramers Gaunt factor $g$ defined according to the formula

$$
\int\left|\int F_{n} \frac{\mathrm{r}}{r^{3}} F_{1} d \mathrm{r}\right|^{2} d \omega=\frac{8 \pi^{2}}{\sqrt{3} k_{1} k_{n}} g
$$

where the wave numbers $k_{1}$ and $k_{n}$ of the incident and

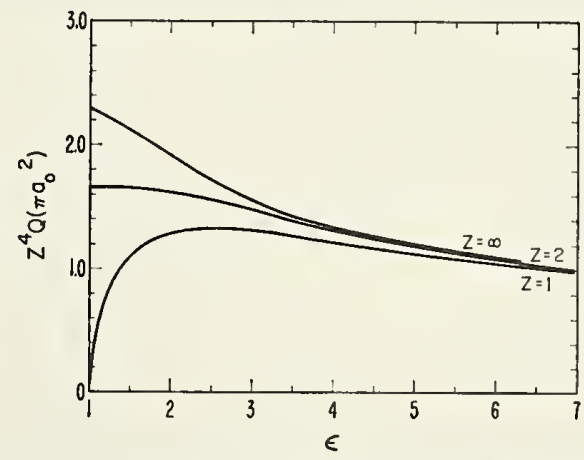

Fig. 41. Total cross section for the $1 s \rightarrow 2 p$ excitation of hydrogenic ions of charge $Z$ calculated using the Coulomb-Born approximation (first Born approximation for $Z=1$ ) (Burgess, 1961). [ $\epsilon$ is electron energy in threshold units defined by Eq. (38).] 
scattered electrons are given in units of $a_{0}^{-1}$, we obtain

$$
\begin{aligned}
Q_{n} & =\left(8 \pi / \sqrt{3} k_{1}^{2}\right)\left[f(n, 1) g / \Delta E_{n 1}\right] & & \left(\text { in } \pi a_{0}^{2}\right) \\
& =\left(1.28 \times 10^{-15} / E\right)\left[f(n, 1) g / \Delta E_{n 1}\right] & & \left(\text { in } \mathrm{cm}^{2}\right),
\end{aligned}
$$

where the unit of energy is the rydberg. This formula yields unsatisfactory estimates of the excitation cross section. It may be improved by introducing a cut-off into the relevant integration in which case $g$ becomes replaced by an effective Gaunt factor $\bar{g}$.

Values of $\bar{g}$ for $1 s \rightarrow n p$ excitations of hydrogen ions are displayed in Table XLIX.

\subsection{Helium Ion}

The hydrogen-like ion which has been the subject of the closest examination is the positive ion of helium corresponding to $Z=2$.

The first detailed investigation of the excitation of $\mathrm{He}^{+}$was carried out by Bransden, Dalgarno, and King (1953) who calculated the zero-order partial cross section for the $1 s \rightarrow 2 s$ excitation using the CoulombBorn approximation, the Coulomb-Born-Oppenheimer approximation and the distorted Coulomb waves approximation both neglecting and including exchange.

The $1 s-2 s-2 p$ close coupling approximation has been used recently by Burke, McVicar and Smith (1964a) and by McCarroll (1964) to calculate the total excitation cross sections for the $1 s \rightarrow 2 s$ and $1 s \rightarrow 2 p$ transitions of $\mathrm{He}^{+}$. Where comparison is possible the two investigations are in good agreement. The partial cross section $Q^{L}$ for the $1 s \rightarrow 2 s$ and $1 s \rightarrow 2 p$ excitations calculated by Burke, McVicar, and Smith (1964a), who carried out the more extensive investigation, are displayed in Tables L and LI, respectively. The values of the triplet and singlet partial cross sections include the spin weighting factors $\frac{3}{4}$ and $\frac{1}{4}$, respectively. It is of interest to note that the partial cross sections calculated neglecting exchange are good approximations to the sums of the triplet and singlet partial cross sections for the $L>4$ partial waves, indicating that exchange can be justifiably neglected for these higher-order partial waves.

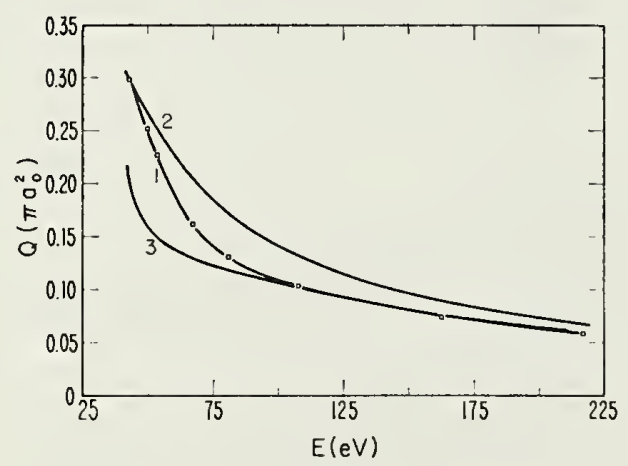

FIG. 42. Total cross sections for the $1 s \rightarrow 2 s$ excitation of $\mathrm{He}^{+}$ ions. Curve 1: $1 s-2 s-2 p$ close coupling approximation (Burke, McVicar, and Smith, 1964a). Curve 2: Coulomb-Born approximation (Tully, 1960). Curve 3: unitarized Coulomb-Born approximation (Burgess, Hummer, and Tully, 1961).

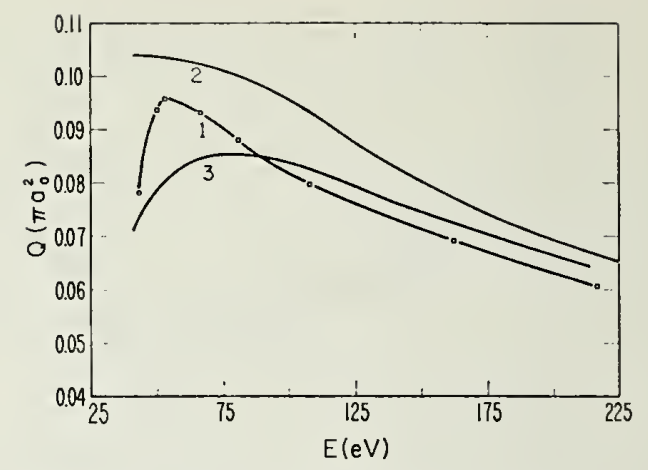

FIG. 43. Total cross sections for the $1 s \rightarrow 2 p$ excitation of $\mathrm{He}^{+}$ ions. Curve 1: $1 s-2 s-2 p$ close coupling approximation (Burke, McVicar, and Smith 1964a). Curve 2: Coulomb-Born approximation (Burgess, 1961). Curve 3: unitarized Coulomb-Born approximation (Burgess, Hummer, and Tully, 1961).

To obtain the total excitation cross sections use was made of the higher $L$ value partial cross sections calculated by Tully (1960) and by Burgess (1961) employing the Coulomb-Born approximation. Very slow convergence with $L$ value was found for the $1 s \rightarrow 2 p$ excitation, it being necessary to include partial cross sections for $L$ values up to 27 for $k_{1}=4$. The $1 s \rightarrow 2 p$ partial cross sections for $L>15$ were obtained by an extrapolation procedure which made use of the fact that the graph of In $Q^{L}$ against $L$ is linear for the higher values of $L$.

The total cross sections for the $1 s \rightarrow 2 s$ and $1 s \rightarrow 2 p$ excitations of $\mathrm{He}^{+}$calculated by Burke, McVicar and Smith (1964a) are displayed in Figs. 42 and 43 where they are compared with the excitation cross section calculated by Tully (1960) and by Burgess (1961) using the Coulomb-Born approximation.

To assess the accuracy of the $1 s-2 s-2 p$ close coupling approximation, Burke, McVicar, and Smith (1964a) included the $3 s$ and $3 p$ states in the close coupling expansion for $k_{1}{ }^{2}=5$ corresponding to $68-\mathrm{eV}$ incident electron energy. The resulting zero-order partial cross sections are given in Table LII where they are compared with the values obtained using the $1 s-2 s-2 p$ close coupling approximation. We see that the effect of allowing for the $3 s$ and $3 p$ states is quite slight for the helium positive ion in contrast to the case of atomic hydrogen for which the analogous effect at $16.5-\mathrm{eV}$ impact energy was found to be considerable as can be verified by referring to Table XXII. Thus it seems that the close coupling approximation has nearly converged to the exact solution with the inclusion of the $1 s, 2 s, 2 p$ states for the helium positive ion. In any event the $1 s-2 s-2 p$ close coupling approximation is certainly better for the case of the helium positive ion than for atomic hydrogen.

Burke, McVicar, and Smith (1964b) have also used the $1 s-2 s-2 p$ close-coupling approximation to calculate the positions and widths of the first few autoionizing states of helium from the resonances which occur in the $S$ and $P$ wave phase shifts for elastic scattering of electrons by $\mathrm{He}^{+}$, these resonances making their appearance by a rapid variation in the appropriate phase shift by $\pi$ 
radians. The calculated positions of these resonances agree very well with the experimental data of Madden and Codling (1963) and Simpson, Mielczarek, and Cooper (1964) as can be seen from Table LIII where a comparison is made for some ${ }^{1} S,{ }^{1} P$, and ${ }^{3} P$ autoionizing levels. It should be remarked however that even when exchange is neglected, the close coupling calculations still provide fairly satisfactory values for the positions of the resonances, which therefore do not seem very sensitive to the detailed assumptions made by the theory.

Calculations of the partial cross sections for the $1 s \rightarrow 3 p$ excitation of the helium positive ion have also been carried out by Burke, McVicar, and Smith (1964b) using the $1 s-3 p$ close coupling approximation. Partial and total $1 s \rightarrow 3 p$ excitation cross sections are displayed in Table LIV.

\subsection{Helium-like Ions}

The Coulomb-Born approximation has been employed by Sural and Sil (1966) to obtain a differential cross section formula for the $1^{1} S \rightarrow 2^{1} S$ excitation of positive ions belonging to the helium isoelectronic sequence using the simple wave functions derived by Morse, Young, and Haurwitz (1935). They carried out detailed calculations for the special case of the $1^{1} S \rightarrow 2^{1} S$ excitation of $\mathrm{Li}^{+}$at the threshold energy. An integration over all angles of scattering gave $0.304 \times 10^{-2} \pi a_{0}^{2}$ for the total cross section at threshold.

\subsection{Lithium-like Ions}

Bely, Tully, and Van Regemorter (1963) have used the Coulomb-Born approximation to calculate the total excitation cross sections for the $2 s \rightarrow 2 p$ resonance transitions of the positive ions $\mathrm{Be}^{+}, \mathrm{C}^{3+}, \mathrm{O}^{5+}$, and $\mathrm{Mg}^{9+}$ all of which belong to the lithium isoelectronic sequence. They employed the simple analytical wave functions derived by Veselov et al. (1961) for the $2 s$ and $2 p$ orbitals of the optical electron. The excitation cross sections obtained by them are presented in Figs. 44-47. Bely (1962) has calculated the effective free-free Kramers Gaunt factor $\bar{g}$ for the resonance transition of $\mathrm{O}^{5+}$ at the threshold energy obtaining $\bar{g}=0.79$.

Bely, Tully, and van Regemorter (1963) also used the $2 s-2 p$ unitarized Coulomb-Born approximation (also known as the Coulomb-Born II approximation) to investigate the resonance transitions of the lithium sequence of positive ions. Only for the $\mathrm{Be}^{+}$ion does the unitarized Coulomb-Born approximation produce significantly different results from those found with the ordinary Coulomb-Born approximation.

A detailed investigation of the $2 s \rightarrow 2 p$ excitation of the $\mathrm{N}^{4+}$ positive ion member of the lithium isoelectronic sequence has been carried out by Burke, Tait, and Lewis (1966) using the Hartree-Fock functions obtained by Weiss (1963). The following approximations were employed:

(i) the Coulomb-Born approximation,

(ii) the unitarized Coulomb-Born approximation,

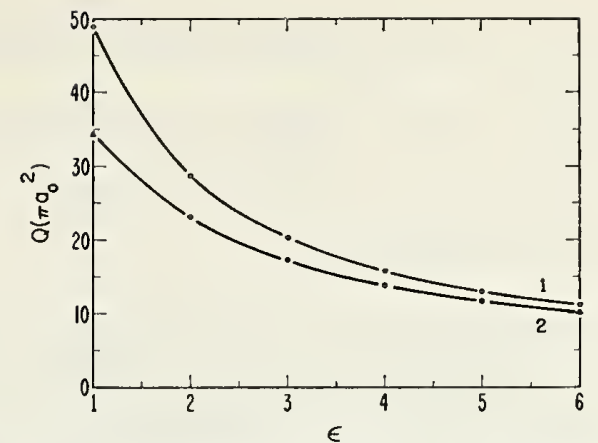

FIG. 44. Total cross sections for the $2 s \rightarrow 2 p$ excitation of $\mathrm{Be}^{+}$ (Bely, Tully, and Van Regemorter, 1963). [ $\epsilon$ is electron energy in threshold units defined by Eq. (38).] Curve 1: Coulomb-Born approximation. Curve 2: unitarized Coulomb-Born approximation.

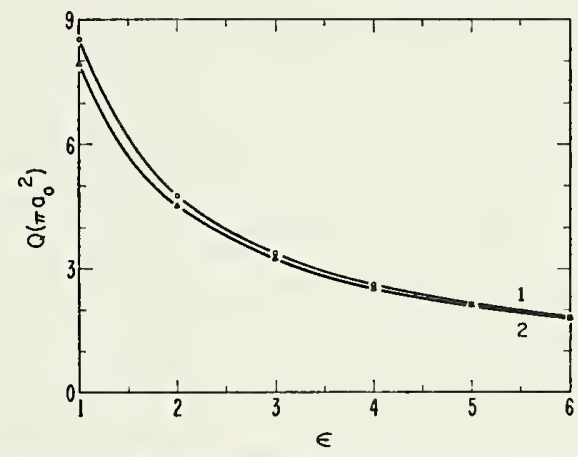

Fig. 45. Total cross sections for the $2 s \rightarrow 2 p$ excitation of $\mathrm{C}^{3+}$ (Bely, Tully, and Van Regemorter, 1963). [ $\epsilon$ is electron energy in threshold units defined by Eq. (38).] Curve 1: Coulomb-Born approximation. Curve 2: unitarized Coulomb-Born approximation.

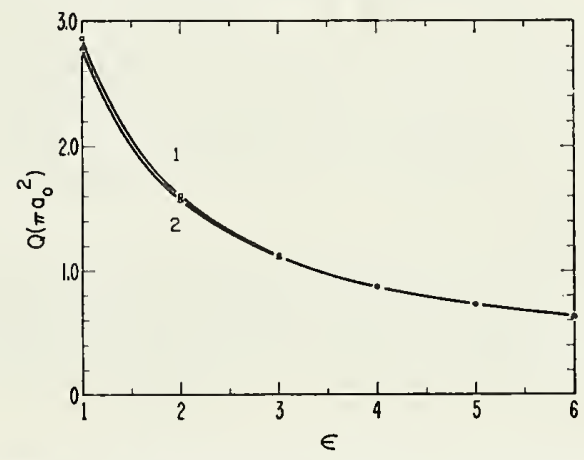

FIG. 46. Total cross sections for the $2 s \rightarrow 2 p$ excitation of $\mathrm{O}^{5+}$ (Bely, Tully, and Van Regemorter, 1963). [ $\epsilon$ is electron energy in threshold units defined by Eq. (38).] Curve 1: Coulomb-Born approximation. Curve 2: unitarized Coulomb-Born approximation.

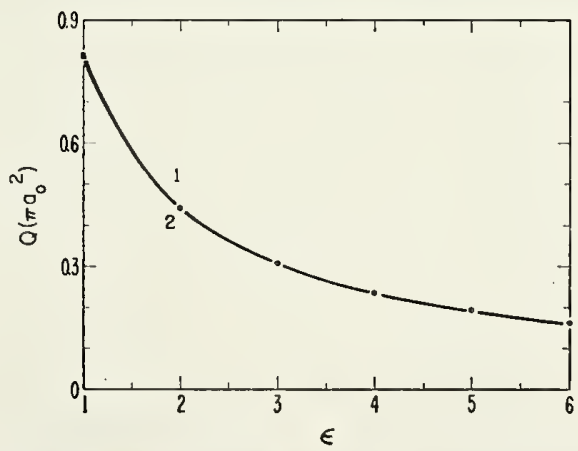

FIG. 47. Total cross sections for the $2 s \rightarrow 2 p$ excitation of $\mathrm{Mg}^{9+}$ (Bely, Tully, and Van Regemorter, 1963). [ $\epsilon$ is electron energy in threshold units defined by Eq. (38).] Curve 1: Coulomb-Born approximation. Curve 2: unitarized Coulomb-Born approximation. 
(iii) the $2 s-2 p$ close-coupling approximation neglecting exchange,

(iv) the $2 s-2 p$ close-coupling approximation including exchange,

(v) the $2 s-2 p-3 s-3 p-3 d$ close-coupling approximation neglecting exchange.

All of these approximations were found to yield the same $2 s \rightarrow 2 p$ excitation cross section within a few percent. The cross section is displayed in Fig. 48. We conclude from this result that the resonance excitation cross sections for the other positive ions of the lithium isoelectronic sequence are also given accurately by the Coulomb-Born approximation, except for $\mathrm{Be}^{+}$whose cross section may be overestimated somewhat.

The $2 s \rightarrow 3 p$ excitation of $\mathrm{N}^{4+}$ was also investigated with considerable thoroughness by Burke, Tait, and Lewis (1966). Although they found that the excitation cross sections given by the Coulomb-Born approximation and the $2 s-3 p$ close coupling approximation with exchange neglected were in good accordance, the effect of allowing for exchange and for the coupling to the $3 \mathrm{~s}$ and $3 d$ states were found to be rather important, both increasing the excitation cross section considerably at the threshold. The application of the unitarized Coulomb-Born approximation gave rise to misleading results since it produced a slight reduction in the $2 s \rightarrow 3 p$ excitation cross section below that given by the ordinary Coulomb-Born approximation. We conclude from this that the predictions of the unitarized Coulomb-Born approximation should be treated with caution and it seems likely that it is a useful approximation only for resonance transitions with large line strengths. The cross sections for the $2 s \rightarrow 3 p$ excitation of $\mathrm{N}^{4+}$ are displayed in Fig. 49.

Burke, Tait, and Lewis (1966) have also employed the Coulomb-Born approximation to calculate excitation cross sections for other transitions connecting the $n=2$ and $n=3$ levels of $\mathrm{N}^{4+}$. These are presented in Table LV.

\subsection{Sodium-like Ions}

The Coulomb-Born approximation has been used by Bely, Tully, and Van Regemorter (1963) to calculate the excitation cross sections for the $3 s \rightarrow 3 p$ transitions

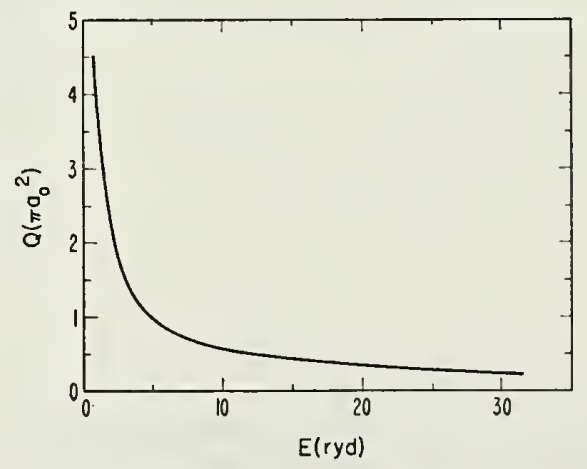

Fig. 48. Total cross section for the $2 s \rightarrow 2 p$ excitation of $\mathrm{N}^{4+}$ calculated using the $2 s-2 p-3 s-3 p-3 d$ close coupling approximation (Burke, Tait, and Lewis, 1966).

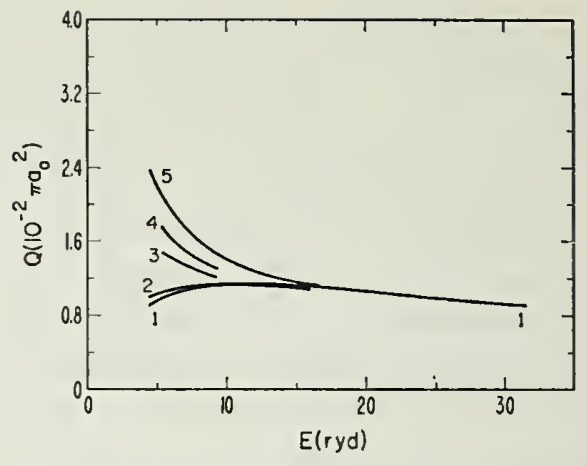

FIG. 49. Total cross sections for the $2 s \rightarrow 3 p$ excitation of $\mathrm{N}^{4+}$ (Burke, Tait, and Lewis, 1966). Curve 1: Coulomb-Born approximation. Curve 2: $2 s-3 p$ close coupling approximation neglecting exchange. Curve $3: 2 s-3 p$ close coupling approximation including exchange. Curve $4: 2 s-2 p-3 s-3 p-3 d$ close coupling approximation neglecting exchange. Curve 5: best estimate of $2 s \rightarrow 3 p$ excitation cross section.

of the positive ions $\mathrm{Mg}^{+}, \mathrm{Si}^{3+}$, and $\mathrm{Fe}^{15+}$ which are all members of the sodium isoelectronic sequence. These excitation cross sections are given in Figs. 50-52. We see that the cross section for the resonance transition of $\mathrm{Mg}^{+}$is decreased considerably by employing the unitarized Coulomb-Born approximation; however for the other ions the reduction is much less important.

Krueger and Czyzak (1965) have carried out calculations on the $3 s \rightarrow 3 p$ and $3 p \rightarrow 3 d$ excitation of $\mathrm{Fe}^{15+}$ using the Coulomb-Born approximation. Their results for the $3 s \rightarrow 3 p$ transition are in close accordance with those obtained by Bely, Tully, and Van Regemorter (1963). The excitation cross section for the $3 p \rightarrow 3 d$ transition of $\mathrm{Fe}^{15+}$ calculated by Krueger and Czyzak (1965) is displayed in Fig. 53.

\subsection{Potassium-like Ions}

The only member of the potassium isoelectronic sequence to be studied so far is $\mathrm{Ca}^{+}$. Van Regemorter (1960a; 1960b; 1961) has calculated total excitation cross sections for the $4 s \rightarrow 4 p, 3 d \rightarrow 4 p$, and $4 s \rightarrow 3 d$ transitions of this positive ion using the Coulomb-Born approximation as well as the unitarized Coulomb-Born approximation. His values are displayed in Table LVI. The total excitation cross sections for the $4 s^{2} S_{1 / 2} \rightarrow$ $4 p^{2} P_{1 / 2}$ and $4 s^{2} S_{1 / 2} \rightarrow 4 p^{2} P_{3 / 2}$ transitions corresponding to the $H$ and $K$ lines of $\mathrm{Ca}^{+}$, respectively, are given by

$$
\begin{aligned}
Q(H)=\frac{1}{3} Q(4 s \rightarrow 4 p) & =19.39 \pi a_{0}^{2} & & k f^{2}=0, \\
& =17.35 \pi a_{0}^{2} & & k f^{2}=0.1, \\
Q(K)=\frac{2}{3} Q(4 s \rightarrow 4 p) & =38.78 \pi a_{0}^{2} & & k_{f}{ }^{2}=0, \\
& =34.70 \pi a_{0}^{2} & & k_{f}{ }^{2}=0.1,
\end{aligned}
$$

where $k_{f}$ is the wave number of the scattered electron.

\subsection{Positive Ions Having $p^{2}, p^{3}$, or $p^{4}$ Configurations}

Seaton (1953b; 1955b; 1956) has carried out a detailed investigation of the excitation of forbidden transitions between the terms of the lowest $p^{2}, p^{3}, p^{4}$ con- 


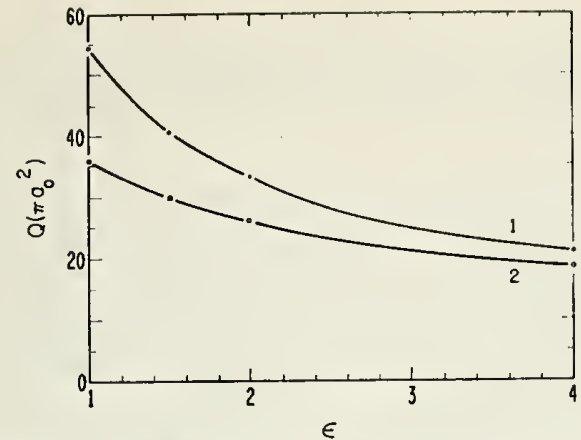

Fic. 50. Total cross section for the $3 s \rightarrow 3 p$ excitation of $\mathrm{Mg}^{+}$ (Bely, Tully, and Van Regemorter, 1963). [ $\epsilon$ is electron energy in threshold units defined by Eq. (38).] Curve 1: Coulomb-Born approximation. Curve 2: unitarized Coulomb-Born approximation.

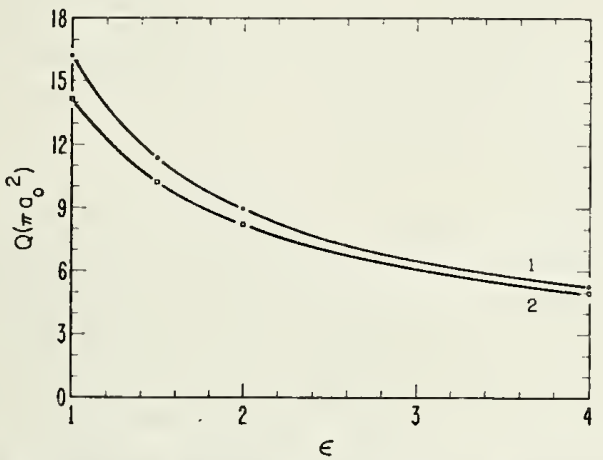

FIG. 51. Total cross section for the $3 s \rightarrow 3 p$ excitation of $\mathrm{Si}^{3+}$ (Bely, Tully, and Van Regemorter, 1963). [ $\epsilon$ is electron energy in threshold units defined by Eq. (38).] Curve 1: Coulomb-Born approximation. Curve 2: unitarized Coulomb-Born approximation.

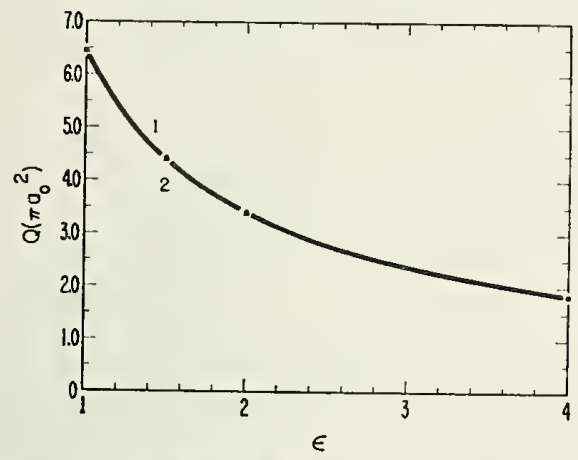

FIG. 52. Total cross section for the $3 s \rightarrow 3 p$ excitation of $\mathrm{Fe}^{15+}$ (Bely, Tully, and Van Regemorter, 1963). [ $\epsilon$ is electron energy in threshold units defined by Eq. (38).] Curve 1: Coulomb-Born approximation. Curve 2: unitarized Coulomb-Born approximation.

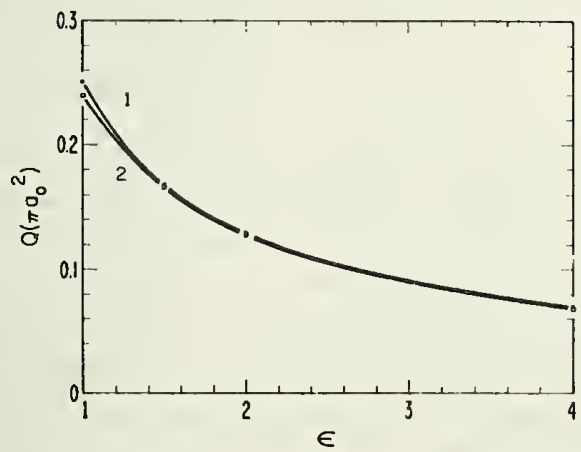

Fig. 53. Total cross section for the $3 p \rightarrow 3 d$ excitation of $\mathrm{Fe}^{15+}$ (Krueger and Czyzak, 1965). [ $\epsilon$ is electron energy in threshold units defined by Eq. (38).] Curve 1: Coulomb-Born approximation. Curve 2: unitarized Coulomb-Born approximation. figurations of certain positive ions. The various terms which occur for these configurations are:

$\begin{array}{ccc}n & p^{2} \text { and } p^{4} & p^{3} \\ 3 & { }^{1} S & { }^{2} P \\ 2 & { }^{1} D & { }^{2} D \\ 1 & { }^{3} P & { }^{4} S,\end{array}$

where we have denoted the terms of a given configuration by $n=1,2,3$ in the order of increasing eigenenergy.

Because the variation with impact energy of the excitation cross section for a positive ion is rather slow, Seaton's calculations were performed at the threshold energy for excitation. The terms corresponding to $n=1,2,3$ very nearly have the same energy for a positive ion and so the basic approximation used by Seaton was the exact resonance approximation which neglects the energy differences between the terms. By far the largest contribution to the collision strengths for the $1 \rightarrow 2$ and $1 \rightarrow 3$ transitions, which both involve spin change and thus include exchange coupling only, is made by the $p$ wave, the other angular momenta providing unimportant contributions. Detailed calculations were performed by Seaton on the positive ions $\mathrm{N}^{+}, \mathrm{O}^{+}, \mathrm{O}^{2+}$, and $\mathrm{Ne}^{2+}$ using Hartree-Fock wave functions. The collision strengths $\Omega(1,2)$ and $\Omega(1,3)$ obtained by Seaton (1953b) employing the exact resonance approximation together with certain small corrections are given in Table LVII. The values given in italics for the other ions were found by interpolation and extrapolation and are estimates only.

The collision strengths for the $2 \rightarrow 3$ transitions of $\mathrm{O}^{+}, \mathrm{O}^{2+}$, and $\mathrm{S}^{+}$ions have also been calculated by Seaton (1955b). Because no change of spin occurs in the $2 \rightarrow 3$ transitions, potential interaction terms arise in the integrodifferential equations describing the excitation collisions. Both potential and exchange interactions have to be taken into account in the case of the $p$ wave. The basic approximation used by Seaton (1955b) for the $p$ wave was the exact resonance approximation, the $\lambda=2$ terms arising from the expansion of the Coulomb interaction $e^{2} / r_{i j}$ between the electrons being allowed for by the use of first-order perturbation theory. The presence of the potential interaction terms results in partial waves other than the $p$ wave being important for $2 \rightarrow 3$ transitions. The collision strengths corresponding to these partial waves were calculated by Seaton (1955b) using the distorted waves approximation but neglecting exchange interaction terms. Values of the total collision strength $\Omega(2,3)$ determined by Seaton (1955b) are displayed in Table LVII.

The exact resonance approximation has also been applied by Czyzak and Krueger (1964) to calculate the collision strengths $\Omega(1,2), \Omega(1,3)$ and $\Omega(2,3)$ for the $3 p^{2}$ ions $\mathrm{S}^{2+}, \mathrm{Cl}^{3+}$, and $\mathrm{Ar}^{4+}$ as well as the $3 p^{3}$ ion $\mathrm{S}^{+}$. Their values for $\mathrm{S}^{+}$differ from those calculated by Seaton (1953b; 1955b), the discrepancy being rather large for $\Omega(2,3)$. Extensive new calculations on the cross sections for the excitation of positive ions having 
ground state $2 p^{q}$ and $3 p^{q}$ configurations have been reported by Saraph, Seaton, and Shemming (1966) and C.zyzak and Krueger (1967).

\section{Polarization of Radiation}

The dipole radiation emitted by an atom after excitation by an electron beam is polarized. Let us suppose that the direction of the incident beam of electrons is parallel to the $0 z$ axis of a rectangular frame of reference $0(x, y, z)$. Then the radiation may be regarded as being due to an electric dipole in the $0 z$ direction and two equal electric dipoles in the $0 x$ and $0 y$ directions. Denoting the intensity of radiation per unit solid angle in a direction perpendicular to the $0 z$ axis due to the electric dipoles parallel and perpendicular to $0 z$ by $I_{\|}$and $I_{\perp}$, respectively, we define the percentage polarization $P$ by the formula

$$
P=100\left(I_{\|}-I_{\perp}\right) /\left(I_{\|}+I_{\perp}\right) .
$$

Then it can be shown that the intensity of radiation per unit solid angle in a direction subtending an angle $\theta$ with the $0 z$ axis is given by

$$
I(\theta)=3\left(100-P \cos ^{2} \theta\right) \bar{I} /(300-P),
$$

where $4 \pi \bar{I}$ is the total intensity resulting from integrating $I(\theta)$ over all solid angles. Since $\bar{I}=I_{\|}+2 I_{\perp}$ we may express the percentage polarization in the alternative form

$$
P=100\left(3 I_{\|}-\bar{I}\right) /\left(I_{\|}+\bar{I}\right) .
$$

The first theoretical treatment of the polarization of radiation was given by Oppenheimer (1927a; 1927b; 1928) and was further developed and applied by Penney (1932b). Recently the theory has been reexamined and extended by Percival and Seaton (1958).

Supposing that the atom can be characterized according to the Russell-Saunders coupling scheme, we let $L^{\prime} S^{\prime} J^{\prime}, L S J$, and $L^{\prime \prime} S^{\prime \prime} J^{\prime \prime}$ be the orbital, spin, and total angular momentum quantum numbers of the initial, upper, and final levels of the atom, respectively. We shall confine our considerations to those instances for which the initial state of the atom has zero orbital angular momentum so that $L^{\prime}=0$.

We first examine the two electron case of helium whose nucleus has zero spin and concern ourselves with the polarization of the light emitted in the line $L S J \rightarrow J^{\prime \prime}$ and in the multiplet $L S \rightarrow L^{\prime \prime} S$. The total spin $S$ of the helium atom can be 0 or 1 . The polarization is zero if the upper level is an $S$ state so that $L=0$. If $L=1$, corresponding to an upper $P$ state, the percentage polarization takes the form

$$
P=100 G\left(Q_{0}-Q_{1}\right) /\left(h_{0} Q_{0}+h_{1} Q_{1}\right)
$$

while if $L=2$, corresponding to an upper $D$ state, we have

$$
P=100 G\left(Q_{0}+Q_{1}-2 Q_{2}\right) /\left(h_{0} Q_{0}+h_{1} Q_{1}+h_{2} Q_{2}\right),
$$

where $Q_{\left|M_{L}\right|}$ is the cross section for the excitation of the state $L S M_{L}$. The values of the constants $G, h_{0}, h_{1}, h_{2}$ obtained by Percival and Seaton using the Oppenheimer-Penney theory are given in Tables LVIII and LIX.

We now examine the polarization of the Lyman $\alpha$ line of hydrogen, produced by the electron impact excitation of the $2 p$ states, and the resonance lines of the alkali metal atoms. These lines all arise from a ${ }^{2} S$ initial state, a ${ }^{2} P$ upper state and a ${ }^{2} S$ final state. For the ${ }^{2} P_{1 / 2} \rightarrow{ }^{2} S$ transition the polarization of the radiation is zero. For the ${ }^{2} P_{3 / 2} \rightarrow{ }^{2} S$ transition and the ${ }^{2} P \rightarrow{ }^{2} S$ multiplet, the percentage polarization according to the Oppenheimer-Penney theory is given by formula (150), the constants $G_{0}, h_{0}, h_{1}$ being presented in Tables LX and LXI. The spin of the atomic nucleus is not necessarily zero for these cases and so the constants involved in the polarization formula were calculated by Percival and Seaton for the values $I=0, \frac{1}{2}, 1, \frac{3}{2}$ of the nuclear spin quantum number.

Unfortunately, the Oppenheimer-Penney theory yields results which depend upon the representation used to characterize the state of the atom. This occurs because the probabilities of exciting the upper states of the atom by electron impact and the optical transition probabilities from these states are calculated independently in the Oppenheimer-Penney theory. The more sophisticated treatment of Percival and Seaton considers the probability of polarized photons being emitted by the whole system of atom and incident electron. As in the simpler theory, the polarization is zero for the $2 p_{1 / 2} \rightarrow 1 s$ transition of atomic hydrogen. For the case of the $2 p_{3 / 2} \rightarrow 1 s$ transition of atomic hydrogen Percival and Seaton find

$$
P\left(2 p_{3 / 2}\right)=100\left(Q_{0}-Q_{1}\right) /\left(1.694 Q_{0}+2.388 Q_{1}\right)
$$

which is quite close to the expression

$$
P\left(2 p_{3 / 2}\right)=100\left(Q_{0}-Q_{1}\right) /\left(1.667 Q_{0}+2.333 Q_{1}\right)
$$

obtained using the Oppenheimer-Penney theory with $I=0$, although rather different from that found taking $I=\frac{1}{2}$. The polarization of the total radiation from the $2 p \rightarrow 1 s$ multiplet of atomic hydrogen is found by Percival and Seaton to be

$$
P(2 p)=100\left(Q_{0}-Q_{1}\right) /\left(2.375 Q_{0}+3.749 Q_{1}\right)
$$

which is not very different from the expression

$$
P(2 p)=100\left(Q_{0}-Q_{1}\right) /\left(2.333 Q_{0}+3.667 Q_{1}\right)
$$

derived using the Oppenheimer-Penney theory with $I=0$ but dissimilar to that obtained with $I=\frac{1}{2}$.

For the case of atomic hydrogen the exact expression for the polarization is close to the Oppenheimer-Penney formula with $I=0$ because the hyperfine structure separations are small. However for atoms other than hydrogen, the hyperfine structure separations are significant and then the theory including hyperfine structure must be employed.

In a recent paper by Flower and Seaton (1967) the effect of allowing for hyperfine structure in the calcula- 
tion of the threshold polarizations of the resonance lines of ${ }^{6} \mathrm{Li},{ }^{7} \mathrm{Li}$, and ${ }^{23} \mathrm{Na}$ has been investigated. The percentage polarizations of the resonance lines calculated using the measured values of the hyperfine structure are given in Table LXII where they are compared with the values determined experimentally by Hafner, Kleinpoppen, and Kruger (1965). The agreement between theory and observation is seen to be very good.

In general the polarization at the threshold energy for excitation can be evaluated without detailed calculations of the cross sections since the scattered electron has zero velocity and therefore zero orbital angular momentum at the excitation threshold so that only states with $M_{L}=0$ can be excited. If we set $Q_{1}=0$ in the Oppenheimer-Penney formula (155) for $I=0$ we obtain $P(2 p)=42.9$ at the threshold for the polarization of the $2 p \rightarrow 1 s$ multiplet while the more elaborate theory of Percival and Seaton yields $P(2 p)=42.1$. However, as a consequence of the close coupling between the $2 s$ and $2 p$ states of hydrogen, this has been shown to be an unjustifiable procedure by Damburg and Gailitis (1963) who find that $P(2 p)$ oscillates near the threshold and does not appear to tend towards a definite limit at the threshold. Their values of $P(2 p)$ near the threshold energy are given in Table LXIII.

At energies above the threshold the polarization of the $2 p \rightarrow 1 s$ multiplet has been calculated by Burke, Schey, and Smith (1963) using their cross sections calculated with the $1 s-2 s-2 p$ close coupling approximation. These values of $P(2 p)$ are also given in Table LXIII.

\section{Theoretical Summary}

The methods which have been employed to calculate excitation cross sections can be conveniently classified according to the strengths of the couplings between the initial and final states of the target atom and between these states and other states of the atom.

For high-electron impact energies the weak coupling approximations are appropriate. They are based upon the assumption that the back coupling of the final state to the initial state, and the couplings of these states to other states of the atom, are small and may be neglected. The most widely used of the weak coupling approximations is the first Born approximation which represents the free electron by a plane wave and may be employed without making undue error for electron impact energies sufficiently far above that for which the excitation cross section attains its maximum value. A still simpler approximation which may be used at moderate as well as high energies is the Bethe approximation which, in the case of optically allowed transitions, replaces the matrix element $V_{n 1}$, coupling the initial state 1 to the final state $n$, by its asymptotic form for large radial distances. A more elaborate weak coupling approximation is the distorted waves approximation which represents the free electron by functions describing its motion in the static fields of the atom before and after the excitation process. The use of this approximation generally leads to an increase in the excitation cross section above that given by the first Born approximation. Neither the first Born nor the distorted waves approximations make allowance for coupling to states other than the initial and final states. Such couplings can be allowed for by using the second Born approximation, provided they are fairly weak, which should be a valid procedure at moderately high impact energies.

The weak coupling approximations discussed so far do not take account of the Pauli exclusion principle and thus neglect the role of electron exchange. Its importance is emphasized by the fact that the Born approximation yields zero for the cross sections for singlet $\rightarrow$ triplet excitations of helium which arise entirely as the consequence of exchange. Now the BornOppenheimer approximation which, like the first Born approximation, represents the free electron by plane waves, is unsatisfactory since it gives rise to excitation cross sections which are gross overestimates at low and moderately high impact energies for the case of $S \rightarrow S$ transitions. The failure of this approximation comes about by the neglect of certain terms of the first order in the interaction energy between the free electron and the atom. If these terms are retained one arrives at the Feenberg or first-order exchange approximation which produces excitation cross sections that are substantially less than those given by the Born-Oppenheimer approximation for $S \rightarrow S$ transitions. An alternative approach is to expand the exchange scattering amplitude given by the Born-Oppenheimer approximation in powers of $k_{1}^{-1}$ where $k_{1}$ is the wave number of the incident electron, retaining only the leading term of order $k_{1}^{-?}$. This is the Ochkur approximation. It appears to yield satisfactory results even for quite low electron energies and has the attractive feature of being no more difficult to apply than the first Born approximation. The distorted waves approximation can also be generalized to take account of electron exchange. Although this is the most accurate of the weak coupling approximations, its application requires an expansion in partial waves and the greatest computational effort.

We now turn our attention to the case of strong coupling between the initial and final states of the target atom but relatively weak coupling between these states and other states of the atom. This case generally arises at low impact energies for transitions between states with a small energy difference, such as the resonance transitions of the alkali metal atoms and the $n s \rightarrow(n+1) p$ transitions of atomic hydrogen for $n$ large. All the weak coupling approximations discussed hitherto fail badly for such cases since they produce excessively large cross sections near the threshold energy. This behavior is a consequence of the fact that the partial cross sections associated with low values of the angular momentum of the incident electron are grossly overestimated by weak coupling approximations in a strong coupling situation, often exceeding the 
theoretically determined maximum value imposed by conservation considerations. Several strong coupling approximations have been introduced by Seaton to overcome this difficulty. The modified Bethe approximation uses the Bethe approximation for the partial cross sections which are less than half the theoretical maximum value $Q_{\max ^{l}}$ and replaces all Bethe partial cross sections which exceed this value by $\frac{1}{2} Q_{\max } \ell$. A somewhat analogous procedure based upon a semi-classical approach and known as the impact parameter method has also been used. Another method of treating strong coupling cases is to employ the exact expression for the excitation cross section given in terms of the reactance matrix $\mathrm{R}$ which is then approximated by using the Born approximation. This automatically ensures that the conservation theorem, or unitarity, is obeyed and has been termed the unitarized Born approximation. Under strong coupling conditions the three approximations discussed above lead to a considerable reduction in the excitation cross section below that given by the Born approximation at low impact energies, and, judging from the case of the $3 s \rightarrow 3 p$ resonance transition of sodium, the results are quite satisfactory. However, if the coupling is insufficiently strong, it should be noted that the very strong coupling approximations may, in certain instances, give rise to misleading results.

If the energy difference between the initial and final states of the transition is very small another approach to the problem may be made. This is called the exact resonance approximation and is based upon the assumption that this energy difference may be taken to vanish thus enabling the coupled equations describing the scattering to be solved exactly. The neglected terms can then be taken into account by an iterative procedure. This method has been applied, for example, to the ${ }^{3} P \rightarrow 1 D$ and ${ }^{3} P \rightarrow 1 S$ excitations involving the terms of the ground configuration of atomic oxygen, and to the $3 s \rightarrow 3 p$ excitation of sodium for which it yields a cross section which is in substantial agreement with that obtained using the modified Bethe approximation and the other strong coupling approximations.

In many instances the coupling between the initial and final states of the target atom may be fairly weak while the coupling of the initial or final state to certain other states of the atom may be strong. An example of such a case is the $1 s \rightarrow 2 s$ excitation of atomic hydrogen for which the coupling between the $1 s$ and $2 s$ states is weak although the coupling of the $2 s$ state to the $2 p$ states, with which it is degenerate, is very strong. Moreover the coupling between the $2 s$ state and the $n p$ states with principal quantum numbers $n \geq 3$ is also strong and can be expected to have an important effect upon the $1 s \rightarrow 2 s$ excitation collision. The most satisfactory approach to the problem of determining excitation cross sections for such cases is to perform an exact solution of the coupled integrodifferential equations connecting the initial and final states and the most important other states of the target atomic system. This is referred to as a close coupling approximation. However it appears that many more states of the atom may make a significant contribution to the scattering than was originally anticipated so that even the $1 s-2 s-2 p-3 s-$ $3 p-3 d$ close coupling calculations performed on the $1 s \rightarrow 2 s$ and $1 s \rightarrow 2 p$ excitations of atomic hydrogen may be some distance from convergence to the actual solution, except for electron impact energies below that required to excite the resonances arising from the $n=4$ level where the 6 state approximation should be quite accurate.

Lastly we note that classical mechanics has also been used to calculate excitation cross sections. The classical approach produces the wrong decay of the excitation cross section with increasing impact energy although it seems to yield cross sections of about the correct magnitude at moderate energies. A synthesis of the classical method and the semi-classical impact parameter method has been introduced which produces excitation cross sections having the correct energy fall off.

\section{EXPERIMENT}

\section{Introduction}

[Note added in proof. In this article we discuss selected measurements from the literature. An extensive compilation of measured electron collision cross sections is being prepared by the JILA Information Center.]

In the study of an atomic collision process the theoretician has the means, in principle, to obtain descriptions adequate for ordinary purposes through application of the Schrödinger equation to the complete physical system. In low-energy atomic physics the quantum-mechanical principles are usually assumed to be fully understood, but the technical difficulties involved in applying them in full to a complete atomic system have been insurmountable. As we have seen in preceding sections it is necessary to simplify the atomic system conceptually and to develop mathematical approximations. These approximations tend to fail in the range below about one hundred electron volts, which is the energy range of greatest interest in the microscopic approach to the study of hot gases (stellar atmospheres, plasmas, etc.).

This regime is then an area of opportunity for the experimental physicist. He may be able to provide much needed data which cannot be calculated accurately or he may be able to provide accurate measurements on a few systems which can be used as a check on the theoretical approximations. In the remainder of this paper we consider the available experimental data on electron impact excitation of atoms, commenting on quality where possible.

The experimentalist, as well as the theoretician, has a problem of simplifying the physical system which he studies. First, it must be simple enough so that it can be 
described accurately and concisely. Second, for purposes of comparison with theoretical work, and to fill the current need for basic microscopic data, the observed phenomena should be a good approximation to the single process usually studied by the theoretician. In this review of experimental work we emphasize those experimental results which seem to be relevant to the isolated electron-atom system. In considering an experimental result, of necessity, the first concern will be with the evidence that the atomic system studied is isolated: (1) that the results are independent of pressure or may be extrapolated reliably to zero pressure, and (2) that the atomic system is not irretrievably influenced by the presence of macroscopic electric and magnetic fields. The general criteria for isolation are discussed further in Secs. 12.1, 12.2, and 12.3. In these sections we have tried to identify and discuss those physical properties of atoms, and of the electron impact excitation process, which must be considered by the experimentalist if systematic errors of serious proportion are to be avoided. This discussion is the heart of the experimental part of the review. It is our contention that with the physical understanding of electron impact excitation now represented in the literature and with the experimental technology now available a very significant improvement in the quality of results could be realized in any future work. The general characteristics of excitation functions have been established. The interest in the immediate future will be in providing more accurate measurements for detailed comparison with calculated cross sections and for applications such as in plasma diagnostics and astrophysical models.

The remainder of the discussion is a rather broad survey of the apparently better experimental results, but few of the descriptions in the literature are complete. The great majority of authors do not mention some of the more important physical and instrumental effects discussed in Secs. 12.1-12.3. In many cases it is clear that the physics involved in the excitation measurements could well have been given more careful attention and measurements could have been more adequately described in publication.

\section{Experimental Methods}

Experimental methods which have been used to study inelastic collisions between electrons and atom fall into three main categories.

The first and most important of these includes those experiments in which intensities of spectral lines excited by an electron beam are used as a measure of the excitation process. The result of such a measurement is a photon-excitation cross section: the probability for producing a photon in a given spectral line, per atom and per unit electron particle flux.

We note at this point that the photon-excitation cross section produced by the experimentalist, even if he succeeds in meeting strict criteria for isolated atoms, in general cannot be directly compared with the level- excitation cross section produced by the theoretician. The differences are due to (1) cascading from higher levels excited by electron impact, (2) the availability of several channels for radiative decay of excited states, and (3) the anisotropic property of the radiation pattern with respect to the electron beam axis [see formula (148)]. The third of these effects is determined by the relative probabilities for exciting the atomic dipoles along the perpendicular to the electron beam axis. Therefore, the photon-excitation cross section as observed in a given direction should be compared with an appropriate theoretical statement written in terms of cross sections for exciting the various magnetic substates of the atom and the transition probabilities which determine the radiative decay scheme.

This distinction between the nature of the primary results of experiment and theory has led to the extensive use of the term optical excitation function. We use this term to describe the dependence on electron energy of the cross section for producing photons in a given line. The term apparent excitation function may be used to describe the excitation function as observed in a particular direction without some correction (such as for anisotropic properties of the radiation pattern).

Figure 54 is a schematic diagram of a typical apparatus used for the measurement of optical excitation functions. Electrons emitted by the heated cathode are passed through the collision chamber in a parallel beam. Usually, the geometry of the electron bean is controlled visually by adjusting anode and grid potentials. In most work the radiation excited by the electrons is sufficient to delineate the electron beam visually. Focussing is usually accomplished purely electrostatically, although a few workers have used axial magnetic fields.

Through the energy range from first appearance of visible light up to the ionization potential the beam is subject to space-charge spreading. However, in most work, the optical system is arranged so the image of the electron beam has its axis perpendicular to the entrance slit of a monochromator and only partially covers the

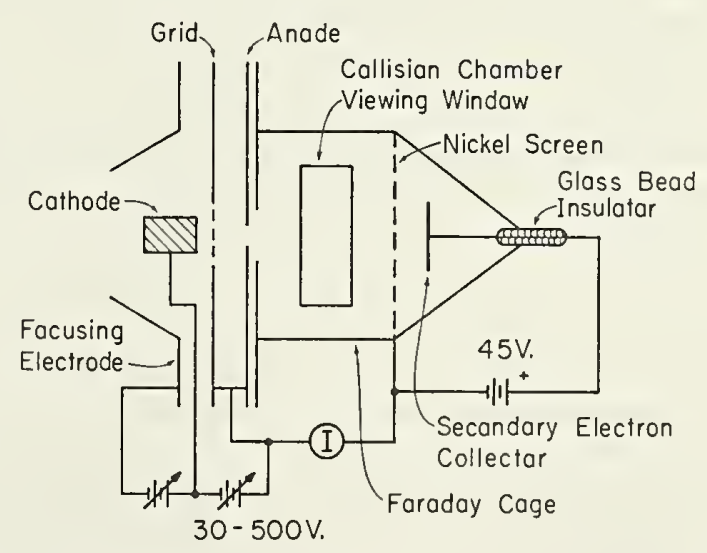

FIG. 54. Electron beam apparatus used for the study of optical excitation functions of helium (St. John, Bronco, and Fowler, 1960). 
slit length. Under these circumstances the signal is usually not noticeably sensitive to minor variations in electron beam diameter.

Above the ionization potential, the beam of ten can be maintained parallel with a diameter defined by the aperture of the electron gun. This evidently occurs when ion densities are sufficient to neutralize the electron beam space charge.

Absolute calibrations of the optical system are carried out by placing a standard tungsten strip lamp in the position of the electron beam, virtually or actually, and calibrating the response of the optical system to the known flux. Absolute calibrations will be discussed in more detail in Sec. 12.4.

The optical method discussed in the preceding paragraphs is only suitable for short-lived states. For states with very long lifetimes a second method based on the ejection of electrons from a metal target has been applied by a number of workers. The metastable atom is deactivated at the target, the excitation energy going into kinetic energy of the ejected electron and into overcoming the work function of the target material. The yield of ejected electrons provides information about the long-lived state similar to that which the optical excitation function provides about the short-lived state. The optical calibration problem is replaced by the problem of determining the efficiency of collection and conversion of metastable atoms at the metal target.

A closely related method is based on deactivation in a gas of lower ionization potential, the Penning ionization process.

The third method for studying inelastic electronatom collisions is through the study of the energy-loss spectrum of scattered electrons. An electron beam of definite energy is passed through a gas. An electron energy-analyzer is used to measure the energy spectrum of electrons scattered at a particular angle with the beam axis. Pressures are reduced to the point that multiple collisions can be neglected.

With the analyzer set to accept electrons of zero energy loss an electron current due to elastic scattering is observed. As the energy loss setting is increased only a background of stray electrons is observed until the energy-loss component transmitted corresponds to the first excitation potential. At this setting a peak in the electron current is observed due to inelastic scattering leaving the atom in the first excited state. Peaks corresponding to excitation to higher excited states can be observed as the energy resolution of the instrument may permit.

The method appears to have some interesting advantages. Where energy resolution permits, an energy-loss component represents the direct excitation to the corresponding excited state, with no complications due to cascading, or due to the lifetime of the excited state. In practice, however, there are difficulties in applying it to the measurement of excitation cross sections as a function of energy. The major problem is in the necessity for taking data at many angles as well as over a range of energies. The angular distribution of electrons must be investigated in detail and used as the basis of integration in order to obtain a total cross section.

Much significant work has been accomplished with this method, nevertheless. Lassettre and his co-workers have used the method, at relatively high incident electron energies, to study angular distributions of inelastically scattered electrons, and some of these results have been used earlier in this paper (3.1) in comparison with theoretical results. Relative magnitudes of differential cross sections for different transitions may also be obtained at a particular angle and energy.

Several groups have exploited this method using high-resolution electron spectrometers at relatively low electron energies (Schulz and Philbrick, 1964; Chamberlain, Simpson, and Kuyatt, 1965) observing peaks corresponding to many doubly excited states lying beyond the ionization limit as well as peaks corresponding to singly excited states. Schulz and Philbrick (1964) and Chamberlain (1965) have carried out highresolution studies of inelastic processes by observing a particular loss component as a function of incident electron energy. These studies do not yield reliable relative cross sections, but they have revealed the occurrence of sharp resonances in the excitation cross sections, which are apparently associated with excitation through intermediate short-lived negative ion states.

\subsection{Pressure Dependence of Excitation Functions}

Our goal in this review is to evaluate the present status of information about the excitation of free atoms by incident electrons. Consequently, we are interested primarily in experiments the results of which are independent of pressure variations or which can be corrected to zero effective pressure. This requirement removes from further consideration many of the older results, including some from well-known works still frequently used.

The major processes which have been recognized as giving rise to pressure dependence of excitation functions are (1) imprisonment of resonance radiation and (2) collisional transfer of excitation. Since much of the following discussion is based on studies of helium excitation, a helium energy level diagram is shown in Fig. 55.

The importance of imprisonment of resonance radiation to measurements of excitation functions was fully recognized by Phelps (1958), who applied the theories of Holstein (1947; 1951), and of Biberman (1947) to the analysis of experimental observations of excitation in helium. In the interpretation of observed excitation functions it is convenient to be able to assume that the photons produced in the de-excitation are subject to a negligible amount of reabsorption in the gas. This is a safe assumption for transitions between excited states, at experimentally practical temperatures, since the populations of excited states are low. Reabsorption in these transitions is negligible. However, for the reso- 


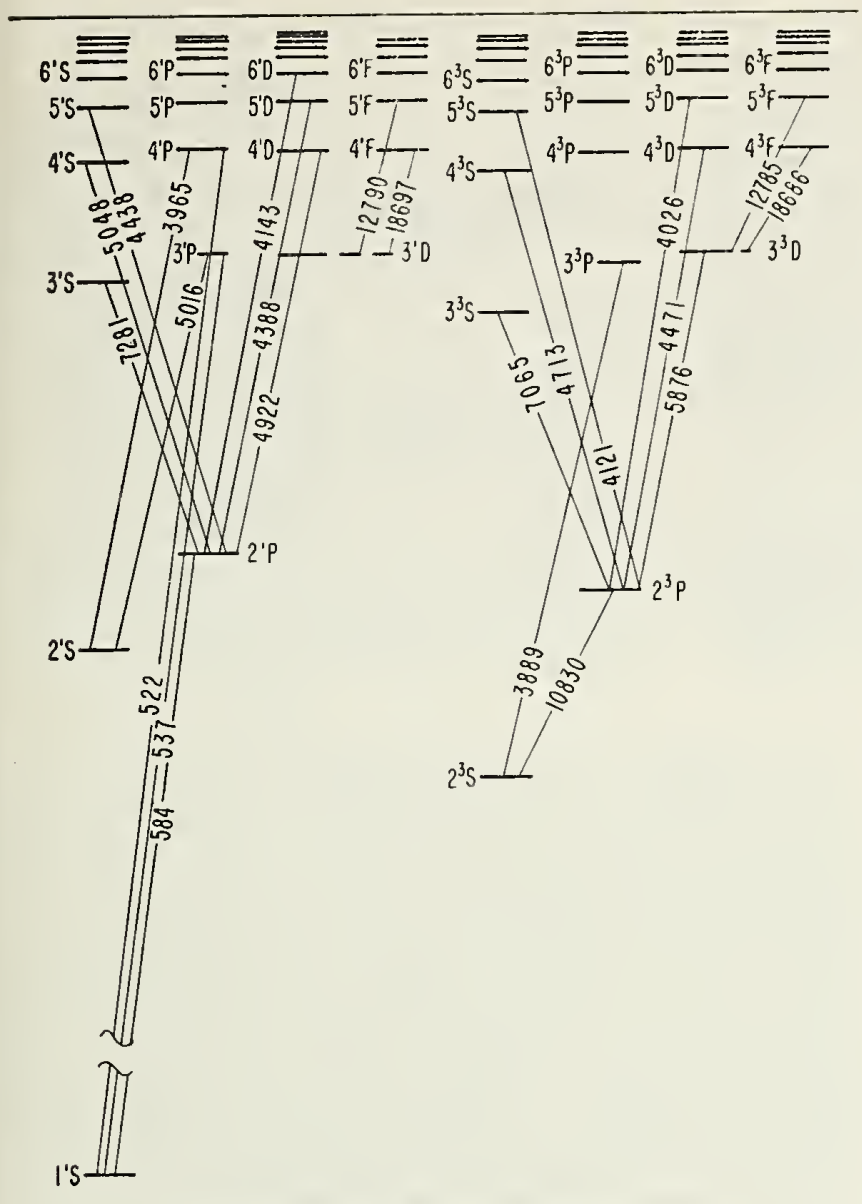

Frg. 55. Term diagram for atomic helium.

nance lines, here defined as those connecting to the ground state, great care is necessary to ensure that the gas density is low enough so that reabsorption is negligible.

Reabsorption of photons by atoms in the ground state effectively lengthens the life of the excited state, and spreads the excited state population over a larger volume. The longer effective lifetime of the upper state results in an increased probability for intervention of collisional processes, and for conversion through radiative transitions to lower levels other than the ground state. In helium, for example, the $3^{1} P \rightarrow 2^{1} S$ excitation function is pressure dependent because of reabsorption in the $3^{1} P \rightarrow 1^{1} S$ line, resulting in conversion of $3^{1} P \rightarrow 1^{1} S$ photons to $3^{1} P \rightarrow 2^{1} S$ photons.

The possible importance of collisional transfer of excitation, as well as of imprisonment of resonance radiation, was considered by Lees and Skinner in 1932 . In collisional transfer, an excited atom is de-excited in a collision with a ground-state atom, with a transfer of excitation energy and possibly changes in the values of angular momentum and spin associated with the excitation energy. An example of such a reaction may be written

$$
\mathrm{He}\left(n^{1} P\right)+\mathrm{He}\left(1^{1} S\right) \rightarrow \mathrm{He}\left(1^{1} S\right)+\mathrm{He}\left(n^{3} F\right) .
$$

In helium the ${ }^{1} P$ levels have large cross sections for excitation by electron impact. Therefore, the most noticeable effect of excitation transfer is the cascading resulting from conversion of $n^{1} P$ state atoms in to states which are nearly degenerate with the $n^{1} P$ state but which are less heavily populated by direct excitation. Thermal energies are available to overcome small energy deficiencies. Lees (1932) observed a change with pressure in the shape of the helium ${ }^{3} D$ excitation function, with the addition of a component similar in energy dependence to the ${ }^{1} P$ excitation functions, this component increasing in size with pressure. He also observer an increase in the volume from which light from ${ }^{3} D$ levels was emitted. A comparable increase was observed in the volume in which radiative decay from ${ }^{1} P$ levels occurred.

Wolf and Maurer (1940) irradiated an isolated sample of helium with helium radiation excited by electron impact. The sample should absorb only in lines connected to the ground state. However, they observed emission from the sample corresponding to transitions from states not optically connected to the ground state including ${ }^{3} D,{ }^{1} D,{ }^{3} P,{ }^{3} S$, and ${ }^{1} S$ states. These effects were explained in terms of direct excitation transfer, providing a convincing confirmation of the excitation transfer theory put forward by Lees and Skinner.

A graphic display of the severe distortion of excitation functions by excitation transfer at moderate pressures was obtained by Lin and St. John (1962). Their results are reproduced in Fig. 56.

There have been a number of investigations of the mechanics of excitation transfer. Gabriel and Heddle (1960) studied the pressure dependence of helium lines excited by electron impact. They noted that in view of the spin conservation rule collisional excitation transfer between singlet and triplet systems at lower levels should be improbable, and they concluded that the increase of population of ${ }^{1} D$ and ${ }^{3} D$ states with pressure was probably from excitation of higher $n^{1} P$ states by way of collisional transfer followed by cascading.

St. John and Fowler (1961) arrived at the same conclusion independently. They noted that the spin conservation rule based on $L S$ coupling, which should preclude intersystem collisional transfer at low values of the principle quantum number, does not rigorously prevent intersystem transfer for the highly excited states for which $L S$ coupling is weak. Furthermore the $3^{3} \mathrm{D}$ level was observed to be particularly sensitive to collisional transfer, and by comparing pressure dependences of different lines they concluded that the dominant transfer mechanism is from ${ }^{1} P$ to ${ }^{3} F$ states at levels $n=4$ through 15 . Lin and Fowler (1961) suggested that a moderately strong selection rule $\Delta L=2$ applies to excitation transfer. Lin and St. John (1962) showed that the pressure dependence of the $4^{1} D$ state population as well as of the $4^{3} D$ state population is consistent with this theory. Kay and Hughes (1967) used time-resolved spectroscopy to study the apparent lifetimes of the $n^{1} P, 3^{1} D, 4^{1} D$, and $3^{3} D$ levels. They 

helium ${ }^{1} D$ and ${ }^{3} D$ excitation functions to excitation transfer effects is illustrated in these curves obtained by Lin and St. John (1962). The abscissas represent electron energy, 0-500 eV. Operating pressures are indicated in millimeters of mercury.
FIG. 56. The sensitivity of the
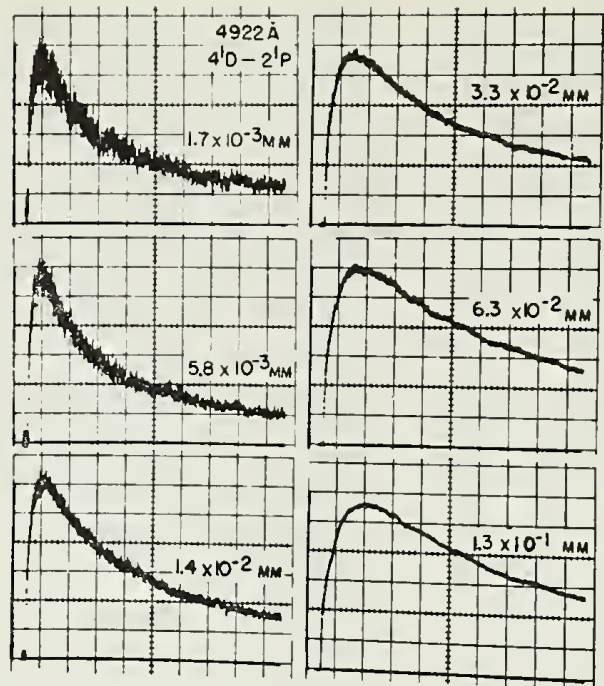

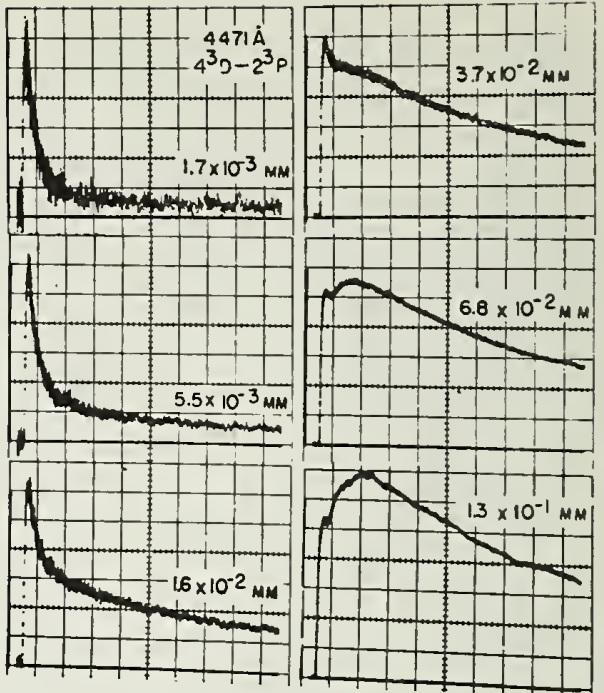

In response to this interpretation, Heddle (1967) concluded that partial breakdown of $L S$ coupling occurs for $n=4$ and is essentially complete for $n \geq 6$. They find that $n^{1} P \rightarrow n F$ cross sections go approximately as $n^{4}$, and that the reverse process is about ten percent of the forward process.

It has been suggested that another mechanism for pressure dependence may be important near threshold. Bogdanova and Geitsi (1964) have discovered that structure in the shapes of some helium excitation functions near threshold is pressure-dependent and, furthermore, may depend on the presence of impurities. Figure 57 shows excitation functions for the helium lines at $4713 \AA\left(4^{3} S \rightarrow 2^{3} P\right)$ and $5876 \AA\left(3^{3} D \rightarrow 2^{3} P\right)$ with mercury impurities present in various concentrations. The helium pressures are about $10^{-2}$ Torr for these curves. The threshold peaks are absent in pure helium, but are evident with $10^{-4}$ Torr of the mercury impurity. Krypton and hydrogen impurities produce similar effects.

Bogdanova, Bochkova, and Frish (1964) have suggested that the threshold pressure dependence is due to the Hornbeck-Molnar process in the following sequence:

$$
\begin{gathered}
\mathrm{He}+e \rightarrow \mathrm{He}^{*}(\mathrm{~A})+e \\
\mathrm{He}^{*}(\mathrm{~A})+\mathrm{He} \longrightarrow \mathrm{He}_{2}{ }^{+}+e \\
\mathrm{He}_{2}{ }^{+}+e \rightarrow \mathrm{He}^{*}(\mathrm{~B})+\mathrm{He} .
\end{gathered}
$$

The last step in this process is rapid for slow electrons, which are available from excitation processes due to electrons of near threshold energies. The relationship of the impurity component to this proposed process is not clear, but it has been noted that the effective impurities have relatively high elastic scattering cross sections for slow electrons and may help confine the low energy electrons to the vicinity of the electron beam. Bogdanova and Marusin (1966) have shown that the pressure-dependent component of the excitation function is delayed in time $\left(\gtrsim 10^{-7} \mathrm{sec}\right)$ with respect to the pressure-independent component. has discussed these threshold effects in terms of space charge due to an intense electron beam. In general, the space charge fields may cause a washing out of structure in the threshold region of an excitation function. The introduction of atoms of low ionization potential may lead to space charge neutralization and permit the observation of structure. Heddle claims that this interpretation is consistent with all available experimental data. He suggests that the time delay observed by Bogdanova and Marusin corresponds to the time necessary to accumulate a positive ion space charge to neutralize the electron space charge. Final resolution of this question will require some additional laboratory work since the evidence is sketchy, but Heddle's explanation, presented with quantitative justification, is much less speculative than the suggestion of Bogdanova and Geitsi.

Multiple scattering of electrons is another pressuredependent-type process which can affect excitation measurements. Elastically and inelastically scattered
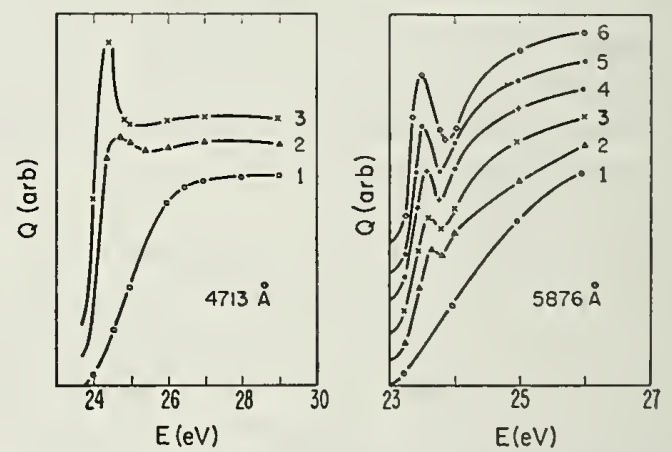

FIG. 57. Threshold behavior of the helium excitation function $Q$ in arbitrary units for the $4713-\AA$ line and the $5876-\AA$ line in pure helium at $10^{-2}$ Torr (curves 1 ) and with partial pressures of mercury ranging from $1 \times 10^{-5}$ to $1 \times 10^{-8}$ Torr (Curves 2-6). The electron beam current density was $7 \times 10^{-4} \mathrm{~A} / \mathrm{cm}^{2}$ and the effective width of the electron energy distribution was about 0.3 $\mathrm{eV}$. To improve the display the curves are displaced successively greater distances upward from the abscissas. [Bogdanova and Geitsi, 1964]. 
clectrons could in principle destroy the spatial definition of the beam and thus cause photometric complications. Such scattered electron current would not have the same axial symmetry as the primary beam so that the state of polarization of radiation excited by scattered electrons would be different from that due to the primary beam. The inelastically scattered electron current, and ionization current if large, would produce excitation characteristic of lower energies than that of the primary electron beam, so that a distortion of the excitation functions would occur.

These effects would be pressure-dependent, and therefore are not important in any pressure range where the excitation function can be shown to be independent of pressure. Since the secondary processes are energy dependent the safe pressure regimes may be expected to vary with electron energy, however.

The number of atoms per $\mathrm{cc}$ at $10^{-3}$ Torr and $293^{\circ} \mathrm{K}$ is $\sim 3.8 \times 10^{13}$. Consideration of typical elastic and inelastic collision cross sections indicates the extent to which gas scattering could be a significant problem at pressures commonly used. The peak of the cross section for ionization of helium is about $3.5 \times 10^{-17} \mathrm{~cm}^{2}$ at $\sim 100 \mathrm{eV}$ (Kieffer and Dunn 1964). Other inelastic processes in helium total approximately $3 \times 10^{-17} \mathrm{~cm}^{2}$ according to Gabriel and Heddle (1960). The probability for inelastic scattering of a $100-\mathrm{eV}$ electron per centimeter of helium at $10^{-3}$ Torr is then of the order of only two-tenths of one percent.

However, for some other atoms the peak ionization cross section is an order of magnitude larger. Excitation cross sections may also be larger. Krypton, xenon, mercury, and the heavier alkalis are anong the atoms having large ionization cross sections, according to Kieffer and Dunn. The electron loss per centimeter through inelastic processes might then amount to several percent. One possible result is a significant attenuation of the electron beam before it reaches the collector, resulting in an absolute error as well as a distortion. Another possible error is the distortion due to excitation by the slow inelastically scattered electrons. Magnetic confinement of radially scattered electrons could help make this effect important.

In the literature there are many examples of measurements demonstrating the sensitivity of excitation functions to pressure-dependent effects. Effects of imprisonment and excitation transfer more or less comparable to those just discussed occur for excitation functions of any atomic species, and a demonstration of the dependence of excitation functions on pressure must be a part of any valid measurement. Much more useful to the reader than the usual statement that signal is proportional to pressure, is a presentation of the apparent excitation as a function of pressure, preferably at several electron energies.

In order to illustrate the nature of effects observed in different lines we show in Figs. 58 and 59 results obtained in helium by Heddle and Lucas (1963). Figure
58 shows the rapid increase with pressure of the magniturle of the apparent excitation function for several helium lines. For $3^{1} P$ excitation the increase is primarily due to imprisonment of $3^{1} P \rightarrow 1^{1} S$ radiation. Collisional transfer loss of $3^{1} P$ atoms is believed to be negligible and the ${ }^{3} P$ population at increased pressure is controlled by the probability for the $3^{1} P \rightarrow 2^{1} S$ transition. At higher principal quantum numbers, however, the excitation transfer collision may be more nearly competitive with the radiative decay loss. This would result in a somewhat shorter lifetime with conversion of ${ }^{1} P$ atoms into other angular momentum states. We note that the primary effect observed would be a change of magnitude.

Of the other excitation functions considered in Fig. 58 , the $4^{3} D \rightarrow 2^{3} P$ function is most pressure sensitive. This observation is consistent with the suggestion of St. John and Fowler (1961) and the theory of Lin and Fowler (1961) to the effect that the ${ }^{3} D$ states are heavily fed by excitation transfer to the ${ }^{3} F$ states with cascading to the ${ }^{3} D$ state.

The $4^{1} D \rightarrow 2^{1} P$ function is subject to the same type of feeding through collisional transfer to the higher ${ }^{\mathrm{t}} F$ states, but the pressure dependence is relatively less because the $4^{1} D$ direct excitation cross section is larger than that for the $4^{3} D$ state (Lin and St. John, 1962). The $4^{3} S \rightarrow 2^{3} P$ and $3^{3} P \rightarrow 2^{3} S$ lines are relatively insensitive to pressure as indicated in Fig. 58. Presumably this insensitivity occurs because the $4^{3} S$ and $3^{3} P$ states are not strongly populated by cascading from the ${ }^{3} \mathrm{~F}$ states. The $4^{1} S \rightarrow 2^{1} P$ line is seen in Fig. 58 to be quite pressure sensitive, as would be expected since the ${ }^{1} S$ states are fed by cascading from the ${ }^{1} P$ states and therefore the apparent cross section would show the indirect effect of imprisonment of resonance radiation. Collisional transfer of excitation is not believed to play a significant role in the pressure sensitivity of this excitation function because of the energy difference between the $4^{1} S$ and $4^{1} P$ states.

The data on polarization of radiation show the same general sensitivities to pressure as do the excitation function data. The curves shown in Fig. 59 indicate that the contributions from secondary processes are effectively depolarized. The pressure dependences of polarization of radiation from several states, notably the $3^{3} P$ and $4^{1} D$ states, in fact, seem to be greater than for the corresponding excitation functions. This probably occurs because the data for excitation and polarization refer to different energies of the exciting electron.

The Heddle and Lucas pressure dependence curves represent the behavior of the excitation functions and of the polarization of helium lines excited at specific electron energies. Pressure dependences of the ${ }^{1} P$ and ${ }^{1} S$ excitation functions due to the trapping of resonance radiation arise through an independent de-excitation mechanism and therefore are independent of energy of the exciting electrons. In the case of the $3^{1} P \rightarrow 2^{1} S$ function of helium, for example, a pressure-independent 
correction to the magnitude may be estimated, if quoted pressures are accurate, on the basis of the theory of imprisonment of resonance radiation.

In general, effects on the final state excitation function of collisional excitation transfer depend on the electron energy. The shape of the excitation function for the initial state is carried over into the final state excitation function. In helium, the ${ }^{1} P$ states which have the largest excitation cross sections, are the major sources for excitation transfer, and the pressure-dependent components of the recipient excitation functions reflect the broad maxima at about $100 \mathrm{eV}$ of the ${ }^{1} P$ functions.

\subsection{The Effects of Polarization}

Most experimental measurements of excitation functions are carried out by observing $I\left(90^{\circ}\right)$, the intensity per unit solid angle of radiation coming off perpendicu-
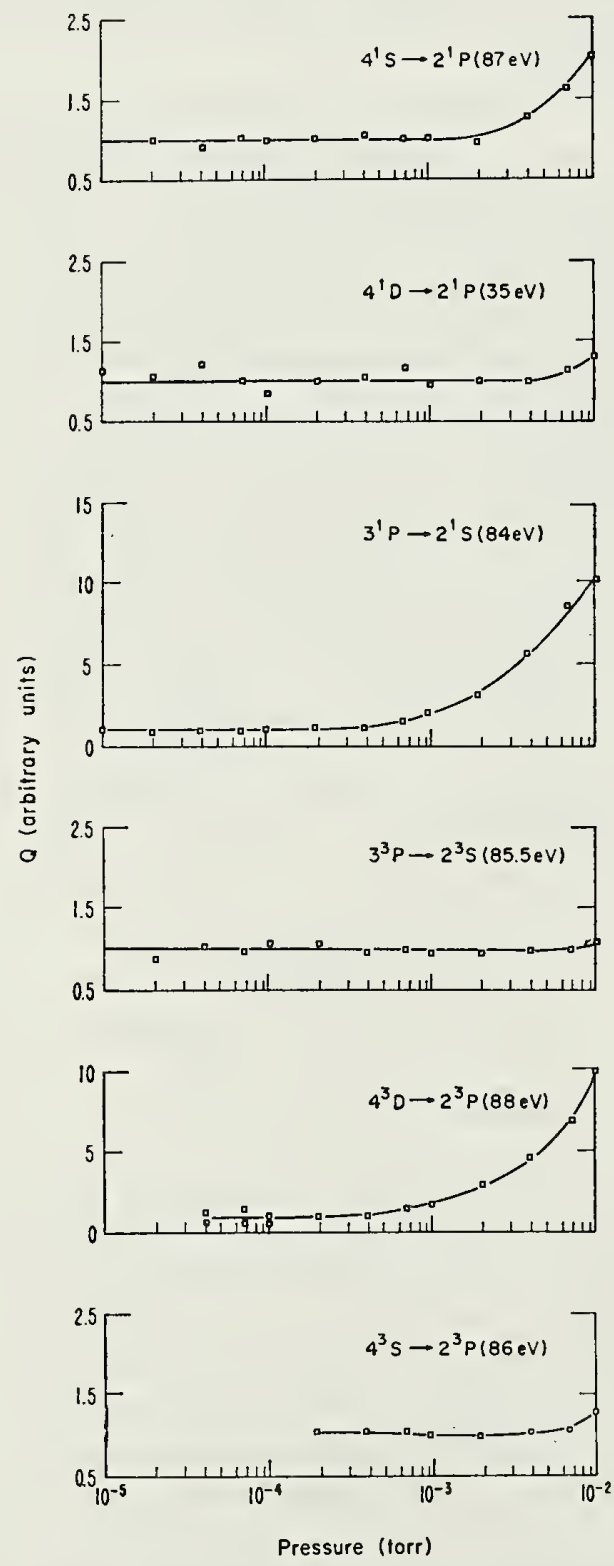

FIG. 58. Pressure dependence of excitation functions for several helium lines. Electron energies used are indicated on the figure (Heddle and Lucas, 1963).
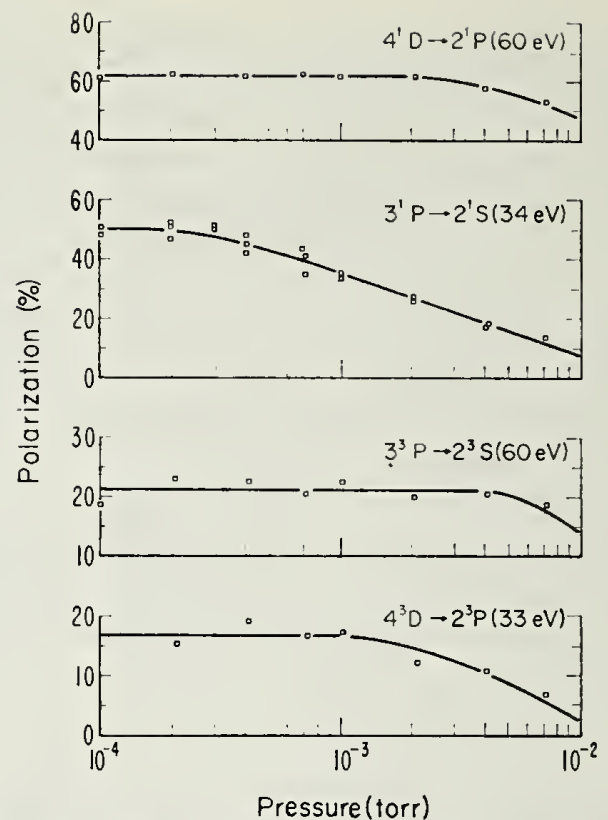

FIG. 59. Pressure dependence of measured polarizations for several helium lines (Heddle and Lucas, 1963).

lar to the electron beam axis. In general, this radiation is polarized and the angular distribution is anisotropic. The theory of polarization of electron impact radiation is discussed in Sec. 9. The angular distribution $I(\theta)$ for electric dipole radiation is related to the percentage polarization as described by Eq. (148)

$$
I(\theta)=3\left(100-P \cos ^{2} \theta\right) \bar{I} /(300-P),
$$

where $\theta$ is the angle of observation with respect to the axis of symmetry provided by the electron beam referred to as the $0 z$ axis in Sec. 9. $\tilde{I}$ is the intensity per unit solid angle averaged over all solid angle. The percentage polarization $P$ is defined by Eq. (147),

$$
P=100\left(I_{11}-I_{\perp}\right) /\left(I_{11}+I_{\perp}\right),
$$

where $I_{\|}$and $I_{\perp}$ are the intensities, observed at $90^{\circ}$ to the electron beam axis, of the components with electric vectors parallel and perpendicular to the electron beam axis.

The polarization of radiation emitted in a transition from a state of definite orbital angular momentum can be expressed in terms of the cross sections for exciting the various magnetic substates $M_{L}$. For example, in the case of excitation of a $P$ state, the cross section for excitation of the $\left|M_{L}\right|=0$ substate is different from the cross section for the $\left|M_{L}\right|=1$ substates. The optical transition probabilities are independent of $M_{L}$, so that the relative values of the cross sections, $Q_{0}$ and $Q_{1}$, determine the angular distribution and the polarization of the emitted radiation.

The primary experimental data on excitation functions are related, ignoring cascading, to some combination of excitation cross sections for the magnetic substates (not to any one of them and not to the total cross section). The specific functional dependence of 
the polarization on the values of $Q\left(M_{L}\right)$ depends on the fine and hyperfine structure. This dependence was discussed in Sec. 9, where expressions are given for the polarization of several lines of helium and hydrogen in terms of $Q_{0}$ and $Q_{1}$. Special cases occur for excitation to the $S$ states, and at threshold for excitation of any level. For $S$ states, with only the substate $\left|M_{L}\right|=0$, the radiation is isotropic and unpolarized. At threshold the outgoing electron carries away no angular momentum. The incident electron only has angular momentum perpendicular to the electron beam axis. Conservation of orbital angular momentum requires that only $\left|M_{L}\right|=0$ states may be excited at threshold from an initial state of zero orbital angular momentum.

The data for $I(\theta)$ can be used to obtain $\bar{I}$ if the polarization $P$ is known. In the case of most experimental data the angle of observation is $\theta=90^{\circ}$ and the expression for $I(\theta)$ reduces to

$$
\bar{I}=I\left(90^{\circ}\right)[1-(P / 300)] .
$$

Equation (158) is the basis for the definition of an apparent cross section $Q_{\perp}$, often used in the discussion of experimental results. $\bar{I}$ bears the same relationship to the total photon-excitation cross section $Q$ which $I\left(90^{\circ}\right)$ bears to $Q_{\perp}$, and

$$
Q=Q \perp[1-(P / 300)] \text {. }
$$

However, use of the "correction" of $Q_{\perp}$ in presenting experimental results tends to reduce the quality of the data, introducing the additional uncertainties of the polarization measurements or calculations. If the primary experimental results are published in addition to values corrected for such effects as polarization and cascading, the maximum worth of the measurement is preserved, independent of changing information about polarization and transition probabilities. Comparisons of theoretical calculations with experimental results may then be based on separate calculations for the cross sections for the magnetic substates, with subsequent comparison with the primary experimental data for $Q_{\perp}$ and with the primary experimental data for the polarization, taking appropriate notice of cascading effects.

The most serious omission found throughout the literature is failure to make proper allowance for instrumental polarization. The observation of the excitation function for a spectral line usually requires the use of a prism or grating monochromator. Usually, the instrument modifies the state of polarization of the light. This may occur, for example, at diffraction gratings where components with electric vectors normal and parallel to the rulings may have different effective transmissions, and at prism surfaces where partial reflection of obliquely incident light may result in modification of the state of polarization.

It can be shown, quite simply, that the ratio of the transmitted intensity to the incident intensity is not constant, but is a function of the state of polarization
$P(E)$ of the incident radiation, which is in turn a function of electron energy $E$. Therefore, the measured excitation function is distorted. The incident intensity $I_{i}(E)$ may be written in terms of components $I_{11}(E)$ and $I_{\perp}(E)$ with electric vectors parallel and perpendicular to the axis of the electron beam. Then

$$
I_{i}(E)=I_{11}(E)+I_{\perp}(E)
$$

and the transmitted intensity

$$
I_{t}(E)=T_{\|} I_{\|}(E)+T_{\perp} I_{\perp}(E),
$$

where $T_{\|}$and $T_{\perp}$ are the transmissions of the instrument for the two components measured separately. It is useful to write, from Eq. (147) and Eq. (160)

$$
\begin{aligned}
& I_{11}(E)=\frac{1}{2}\left[I_{i}(E)\right]\{1+[P(E) / 100]\} \\
& I_{\perp}(E)=\frac{1}{2}\left[I_{i}(E)\right]\{1-[P(E) / 100]\} .
\end{aligned}
$$

Then use of Eqs. (162) and (163) in (161) leads to

$$
I_{t}(E)=I_{i}(E) \frac{1}{2}\left(T_{\|}+T_{\perp}\right)\left[1+\left(\frac{T_{\| 1}-T_{\perp}}{T_{\| 1}+T_{\perp}}\right) \cdot \frac{P(E)}{100}\right] \text {. }
$$

The second term in the square brackets represents an energy dependence in the ratio of the transmitted to the incident intensity. If $T_{\|} \neq T_{\perp}$ and $P(E) \neq 0$, the measured intensity is not proportional to the excitation function. The fractional error in the measured excitation function [in addition to the effect described by Eq. (158)], is

$$
\Delta I_{t} / I_{t}=\left(T_{\|}-T_{\perp}\right) /\left(T_{\|}+T_{\perp}\right) \cdot P(E) / 100 .
$$

This error can be very large, in fact, since severe instrumental asymmetry is known to occur.

It is important to notice in this connection that the instrumental transmission coefficients $T_{\|}$and $T_{\perp}$ are functions of wavelength, and that the instrumental error will be different, in general, from one spectral line to another. Figure 60 shows the polarization of natural light produced by a half-meter Ebert-type grating spectrograph blazed at $4000 \AA$. Other examples are given in a recent review by Heddle (in press).

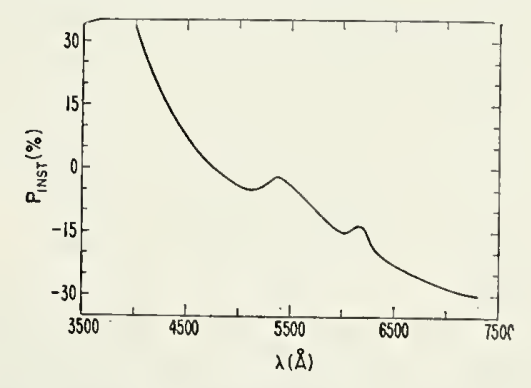

Fig. 60. Instrumental polarization

$$
\left(T_{\max }-T_{\min }\right) /\left(T_{\operatorname{ma}}+T_{\min }\right)
$$

of a half-meter Ebert-type grating spectrograph, calculated from measurements by G. Dunn (private communication). 
Various devices for depolarizing monochromators can be applied. One method uses a partially polarizing transmission element, such as an oblique quartz plate, which is adjusted at each wavelength to the angle at which it exactly compensates for the instrumental polarization. Another method relies on rotation of the axis of polarization of the incident light by an angle such that $T_{11}=T_{\perp}$. This method also requires readjustment at each wavelength, and may be erroneously applied for some longer-lived states if rotation of the axis of polarization by stray magnetic fields is neglected [see Eq. (171)].

A method avoiding the anisotropy associated with polarization is to observe the radiation, not at $90^{\circ}$ to the electron beam, but at the "magic angle," that angle at which the total intensity is equal to the average intensity over the total solid angle. From Eq. (148) we may write this condition

$$
I\left(\theta_{M}\right)=\bar{I}
$$

or

$$
3\left(100-P \cos ^{2} \theta_{M}\right)=300-P
$$

which leads to $\cos \theta_{M}=(\sqrt{3})^{-1}$ and $\theta_{M}=54^{\circ} 46^{\prime}$. However, the polarization of light emitted at angle $\theta$ is related to the polarization of light emitted at $90^{\circ}$ through the expression

$$
\frac{P(\theta)}{100}=\frac{\left[I_{11}\left(90^{\circ}\right)-I_{\perp}\left(90^{\circ}\right)\right] \sin ^{2} \theta}{I_{11}\left(90^{\circ}\right) \sin ^{2} \theta+I_{\perp}\left(90^{\circ}\right)\left(\cos ^{2} \theta+1\right)}
$$

and this is finite at $\theta_{M}=54^{\circ} 46^{\prime}$. Therefore, there remain the problems of instrumental polarization.

Burrows and Dunn (private communication) have suggested a more complete solution: to observe at $90^{\circ}$ to the electron beam axis but to permit only one component of polarization, at $35^{\circ} 14^{\prime}$ to the electron beam axis, to pass into the monochromator. A Polaroid sheet is used to accomplish this. As a result, the light incident on the monochromator is composed of two polarizations according to the relation

$$
I_{i} \propto I_{\| 1} \cos ^{2} \theta_{M}+I_{\perp} \sin ^{2} \theta_{M}=\frac{1}{3} I_{11}+\frac{2}{3} I_{\perp}=\frac{1}{2} \bar{I} .
$$

The response is proportional to the total cross section. Furthermore, since the radiation is linearly polarized at a fixed angle, $\theta=35^{\circ} 14^{\prime}$, the output is independent of instrumental polarization effects. The method by-passes almost all the problems arising from polarization and permits a direct measurement of the total cross section, but information about polarization is lost. Again there is a possible error for the long-lived states due to Larmor precession about residual magnetic fields.

Measurement of polarization requires measurement of the individual components $I_{\|}$and $I_{\perp}$. This is accomplished by use of an analyzer in the light beam, ahead of the monochromator. The observed polarization $P_{\text {cbs }}$ is then expressed in terms of the transmitted signals $I_{\| 1} T_{\|}$and $I_{\perp} T_{\perp}$,

$$
P_{\text {obs }} / 100=\left(I_{1 \mid} T_{\|}-I_{\perp} T_{\perp}\right) /\left(I_{\|} T_{\|}+I_{\perp} T_{\perp}\right) .
$$

Use of Eqs. (162) and (163) leads to the form

$$
\frac{P_{\text {obs }}}{100}=\frac{(P / 100)+\left[\left(T_{11}-T_{\perp}\right) /\left(T_{11}+T_{\perp}\right)\right]}{1+(P / 100) \cdot\left[\left(T_{11}-T_{\perp}\right) /\left(T_{11}+T_{\perp}\right)\right]} .
$$

where $P$ is defined by Eq. (147). The possibility for errors due to instrumental effects is clearly indicated by Eq. (169). Successful measurement of the polarization $P$ requires depolarization of the instrument at each wavelength or an explicit correction for instrumental transmission asymmetry, based on Eq. (169). Heddle (private communication) has suggested that depolarization of the instrument may be accomplished using a polaroid analyzer set parallel or perpendicular to the electron beam axis, but preceded by a half-wave plate which may be rotated so that the analyzer passes either $I_{\| 1}$ or $I_{\perp}$ through the monochromator. Both components are subject to the same instrumental transmission in this method and the error is eliminated. However, a different half-wave plate is required at the wavelength of each excitation function studied.

As has been suggested in the preceding paragraphs Larmor precession is potentially an important source of systematic error. Precession, about residual magnetic fields, of atoms with nonzero orbital angular momentum may result in depolarization, and in rotation of the polarization axis. The Larmor precession frequency is

$$
L=g \mu_{0} H / 2 \pi \hbar=1.4 \times 10^{6} \mathrm{gH} \mathrm{cps},
$$

where $\mu_{0}$ is the magnetic moment of one Bohr magneton, $g$ is the Lande $g$ factor, $2 \pi \hbar$ is Planck's constant, and $H$ is the magnetic field in gauss. Since $g$ is approximately unity and the terrestrial magnetic field is about $0.5 \mathrm{G}$, the angular precession frequency, $2 \pi L$, may well be of the order of $10^{7} \mathrm{rad} / \mathrm{sec}$. The mean lifetimes of a number of excited states are $10^{-7} \mathrm{sec}$ or longer so that significant disorientation will occur if there is a large unattenuated component of the earth's field or some other field perpendicular to the beam axis.

For some experimental work magnetic fields are used to confine the electron beam. If these fields are homogeneous and aligned with the electron beam and are large compared to the earth's field no disorientation occurs. For inhomogeneous fields $|d H / d r|=\frac{1}{2}|d H / d z|$ and disorienting radial fields may become significant off the axis. On the other hand, most experimental work does not involve deliberate use of magnetic fields. In fact magnetic shielding is frequently used to minimize deflection of the electron beam. This is especially common for measurements involving elements with low excitation thresholds. For the electron in a magnetic field $H$ the radius of curvature is $3.36(e V)^{1 / 2} / H \mathrm{~cm}$, where $e V$ is the electron energy in electron volts. For situations in which electron energies of interest are not too low it is common practice to accept a small residual field: terrestrial and stray fields of as much as half a gauss could be tolerated in some work without undue curvature of electron trajectories. 


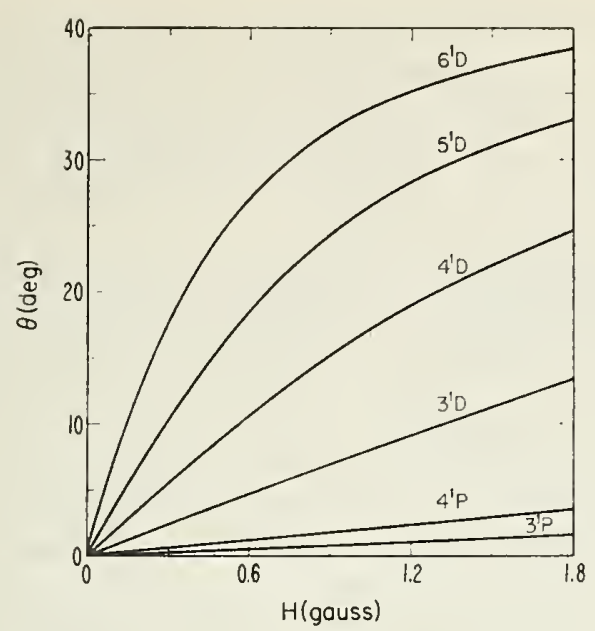

FIG. 61. Rotation of the axis of polarization about a magnetic field perpendicular to the axis of the exciting electron beam, shown for several states of helium as a function of magnetic field. Observation is along the magnetic field. The curves are calculated from Eq. (171) of the text.

Methods of calculating the effect of Larmor precession on polarization are readily available in the literature (Ruark and Urey, 1930, p. 359). For the special case of a residual magnetic field perpendicular to the electron beam along the direction of observation, the axis of polarization is rotated through angle $\Phi$, where

$$
\tan 2 \Phi=4 \pi L / \Gamma \text {. }
$$

Here $\Gamma$ is the reciprocal lifetime of the excited state. The precession is accompanied by a decrease in polarization of the observed radiation given by

$$
P / P_{0}=\left[1+(4 \pi L / \Gamma)^{2}\right]^{-1 / 2},
$$

where $P_{0}$ is the percent polarization in the absence of a magnetic field. The effect is illustrated in Figs. 61 and 62 showing the rotation of axis and the depolarization of the $n^{1} D$ states of helium. For states which live as long as the $6^{1} D$ states, residual magnetic fields may cause serious errors. On the other hand, short-lived states connecting to the ground state are not significantly affected by precession.

\subsection{Electric Field Effects}

Stray electric fields exist in any practical electron beam apparatus. These may include space-charge fields due to the electron beam, especially at high currents and in magnetically confined beams; fields due to contact potentials; fields due to charges accumulated on insulating surfaces; and penetration of fields originating in electrostatic electron optical elements. The question of residual electrostatic fields is a difficult and controversial one.

The magnitudes of fields to be expected from space charge in an unconfined beam can be estimated from a simple calculation based on an infinitely long beam of uniform density. In this case

$$
E_{\mathrm{radia}} \simeq 0.3\left[I_{\mu \mathrm{A}} /(\mathrm{eV})^{1 / 2}\right]\left(R_{\mathrm{mm}}\right)^{-1} \mathrm{~V} / \mathrm{cm}
$$

at the outer edge of the beam and has a linear dependence on radius within the beam, vanishing at the origin. Here $\mathrm{eV}$ is the electron energy in electron volts, $R_{\mathrm{mm}}$ is the beam radius in millimeters, and $I_{\mu \mathrm{A}}$ is the beam current in microamperes. The volume average field within the homogeneous beam is two-thirds of $E_{\text {radial }}$ at the outer edge. From the experimental conditions described in the literature it is clear that radial fields of one or two volts per centimeter are commonly tolerated, and substantially higher fields may be achieved. These electron space-charge fields may be effectively canceled by positive ion space charge for energies above the ionization threshold. The problem of space charge has been discussed in greater detail by Heddle (1967).

Jongerius (1961) has noted that a significant distortion of the electron energy scale may occur at the ionization potential. This is due to the process just cited whereby the positive ion space charge may build up to a level at which the electron space charge is neutralized. Within a small range of applied accelerating potential the actual electron energy may change by more than a volt as the negative space-charge depression is overcome. Jongerius presented this in connection with a study of the dependence of mercury excitation functions on gas pressure and electron beam density.

Bethe and Salpeter (1957, p. 284ff) have discussed the mixing of orbital angular momentum states of atomic hydrogen by electric fields. Because of the degeneracy of states with different orbital angular momenta but equal total angular momenta, very small electric fields suffice for mixing in atomic hydrogen. For the $j=m=\frac{3}{2}, n=3$ levels electric fields must be

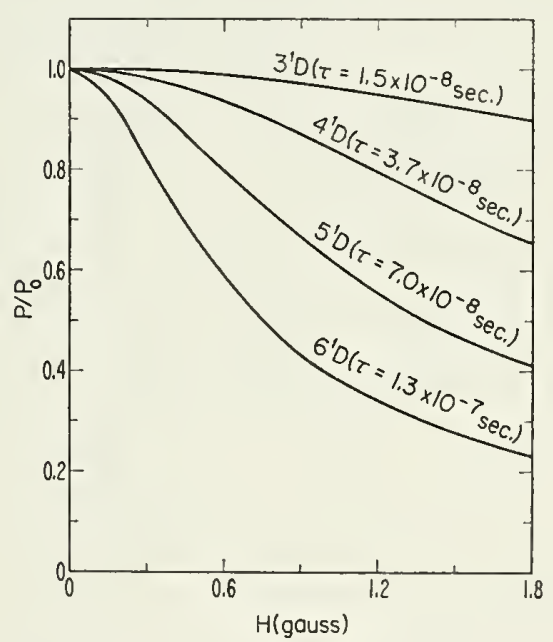

FIG. 62. Depolarization of radiation excited by electron impact, due to Larmor precession, shown for several states of helium as a function of the magnetic field component perpendicular to the electron beam. Observation is along the perpendicular magnetic field component. The curves are calculated from Eq. (172). The mean natural lifetime $\tau$ for each of the ${ }^{1} D$ states is indicated on the figures. 
much less than $F_{c}=1.9 \mathrm{~V} / \mathrm{cm}$ to avoid mixing. Critical vaiues of the field strengths, $F_{c}$, fall off roughly as $n^{-5}$, so mixing in higher $n$ values is certainly important in any experimental apparatus. The critical field strengths for mixing of the $j=\frac{1}{2}$ states is much larger because of the Lamb shift, but even here the critical field falls as $n^{-5}$ and is down to $\sim 1.7 \mathrm{~V} / \mathrm{cm}$ for $n=6$.

In other atoms the degeneracies are removed in the lower levels, but the very high levels are likely to be subject to broadening and mixing by small fields. However, for most lines amenable to study, the effect of electric field mixing on lifetimes and polarization of lines can probably be neglected except for atomic hydrogen.

\subsection{Absolute Measurements of Optical Excitation Functions}

The determination of absolute values for optical excitation measurements may be accomplished by calibrating the optical system using a tungsten strip lamp. It is important that this standard lamp be unpolarized. A calibrated lamp of known spectral radiance may be used, or the spectral radiance may be calculated from Planck's law if the temperature of the strip can be accurately determined with an optical pyrometer. The use of pyrometers and strip lamps is discussed in some detail by Kostkowski and Lee in NBS Monograph 41 (1962), entitled "Theory and Methods of Optical Pyrometry." That paper adequately points out the difficulties of attaining precision and accuracy in these calibrations, and emphasizes the considerable care and experience which must accompany the use of strip lamps and pyrometers. Optical pyrometry is an involved subject and is not discussed in detail here. However, disappearing filament-type pyrometers currently calibrated in a national laboratory and used with care may give results accurate within about $\pm 7^{\circ} \mathrm{C}$. Since pyrometers are universally calibrated on the International Practical Temperature Scale, a correction to the Thermodynamic Temperature Scale is necessary. Radiant flux in the visible spectrum from a tungsten strip lamp, calculated on the basis of such temperature measurements has associated errors of about $\pm 5 \%$, allowing for about $2 \%$ uncertainty in the emissivity of tungsten. This is a statement of minimum error based on a current, high quality calibration and skilled operation. Pyrometers not currently calibrated may well be several times worse because of drift in pyrometer lamps. Pyrometers calibrated through additional transfers from national standards are subject to additional errors.

There remains the problem of illuminating the optical system to be calibrated with the same effective pattern of radiation as is provided by the electron beam source. The usual technique is to place the strip lamp at the position of the electron beam. The same condenser lens may be used to focus the radiation from the strip lamp onto the monochromator entrance slit as is used for the electron beam source. In this case the effective solid angle presented to the source by this lens need not be known, but it must be small enough so that the optics within the monochromator are not exceeded in either mode of illumination.

The calibration is achieved by comparing the signal output produced by the radiation from that area of the tungsten strip which is focused through the monochromator entrance slit, with the signal produced by the volume of electron beam from which radiation is transmitted by the entrance slit. No general statement of the geometrical calibration factor can be given since the geometrical analysis varies with the exact arrangement of the optical system. The electron beam and tungsten strip are usually perpendicular to the slit, and the images do not cover the slit length. The source lengths contributing to transmitted radiation are determined by the slit width and the linear magnification.

The tungsten strip width and Wien's law are used to calculate the radiant flux per unit source length per unit solid angle per unit bandwidth. Multiplication by the monochromator bandwidth, the product of exit slit width and reciprocal dispersions, yields radiant flux per unit solid angle per unit source length contributing to the detector signal. This number permits the signal output from the electron beam excitation mode to be converted to total radiant flux per unit solid angle per unit length of electron beam. If the gas density, polarization of the radiation, and total electron current are known, the radiant flux emitted per unit solid angle can be converted to an absolute photon excitation cross section. The electron beam diameter and current distribution are irrelevant for the geometrical analysis, as are the dimensions of the optical system and an exact knowledge of the effective source length.

If different slit widths are used with the two sources, then the source lengths differ and the ratio of source lengths must be accurately known. Also if removable reflectors are used so that the calibrated lamp can be placed virtually at the position of the electron beam then the effect of extra reflection losses must be considered.

Several points at which measurement limitations are likely to affect the accuracy of the absolute measurements are:

(1) Determination of radiant flux. This always is of the order of $\pm 5 \%$ in the best of circumstances and is entirely likely to be much larger. Photoelectric pyrometers, capable of more accurate results, have not yet been applied in this field.

(2) Gas density measurement. This problem has been discussed recently by Kieffer and Dunn (1966) in a critical review of ionization measurements. They emphasize that density measurement techniques are in a very unsatisfactory state. Determination of density to 
within a few percent is impossible and there are indications that errors of 5 and $10 \%$ are common.

(3) Determination of angular distribution of radiation from electron beam excitation.

(4) Determination or elimination of instrumental polarization $P_{\text {inst.. }}$.

(5) Photoelectric detectors usually are not uniformly sensitive over the receiver surface. Small systematic changes of electron beam geometry may cause errors.

\subsection{Electron Beam Measurements}

Kieffer and Dunn (1966) have discussed many of the problems associated with measurements based on the use of electron beams in static gases. Much of their discussion is relevant for excitation function measurements, and will not be repeated in this review. Accurate electron beam measurements depend on proper precautions with respect to such effects as gas scattering, reflection, secondary emission, and ionization, which can cause incomplete collection of the electron beam or false electron currents. These effects are energy-dependent so they may result in errors in shapes as well as absolute values of excitation functions, and are as important here as they are for the ionization measurements discussed by Kieffer and Dunn.

\section{Other Reviews of Electron Impact Excitation}

Among the various reviews of electron-impact excitation work which have been written, two are of special interest here:

"The Measurement of Optical Excitation Functions" by D. W. O. Heddle, in Methods of Experimental Physics, L. Marton, Ed. (Academic Press Inc., New York, to be published), Vol. 8.

"Measurements of Electron Excitation Functions" by D. W. O. Heddle and R. G. W. Keesing, in Advances in Atomic and Molecular Physics, D. R. Bates and I. Estermann, Eds. (Academic Press Inc., New York, 1968), Vol. 4.

The spirit of these reviews is much the same as that of the preceding sections. The authors are intent upon elucidating the physical and instrumental effects which must be controlled in high-quality measurements of excitation functions.

Some other experimental reviews of interest are:

"Excitation and Ionization of Atoms by Electron Impact," by H. S. W. Massey, Handbuch der Physik, S. Flügge, Ed. (Springer-Verlag, Berlin, 1956), Vol. XXXVI, Atoms II.

"The Measurement of Collisional Excitation and Ionization Cross Sections," by W. S. Fite, in Atomic and Molecular Processes, D. R. Bates, Ed. (Academic Press Inc., New York, 1962).
These latter will serve the reader as excellent supplements to the sections to follow, in which we attempt to summarize and discuss excitation functions to be found in the literature for atomic hydrogen, atomic helium, the noble gases, and the atoms for which the unfilled shells are one and two electron structures.

\section{Atomic Hydrogen}

The excitation of atomic hydrogen has received much more attention from the theoretical physicist than has the excitation of any other atomic species, due to the relative simplicity of the system and the availability of accurate free-atom wave functions. The experimental situation is not quite so fortunate in this respect. It is helium, rather than hydrogen, which is convenient for experimental purposes. The technical problem of producing dissociated hydrogen in the laboratory in an adequately pure state and in sufficient concentration has provided a barrier to experimental work with atomic hydrogen.

Some early work on the excitation of atomic hydrogen was carried out by Ornstein and Lindeman (1933), who recorded the relative intensities of the first three Balmer lines as a function of bombarding electron energies. The atomic hydrogen was produced in a Woods discharge in hydrogen gas. Atom rich hydrogen was pumped out through a side-tube across the path of an electron beam. Light excited in the region of electronatom interaction was studied with a spectrograph using photographic recording. The authors recognized the major difficulty in this method: recombination of atomic hydrogen on metallic surfaces is so effective that the use of electrodes to define potentials in the interaction region was precluded.

No further progress was made for more than twenty years. In 1958 Fite and his collaborators published a series of papers describing work accomplished using beams of electrons and hydrogen atoms crossed in high vacuum. The excitation work presented at that time (Fite and Brackmann, 1958) was based on the observation of Lyman- $\alpha$ flux from the electron-atom interaction volume. This region was shielded from electric fields in order to avoid quenching the long-lived $2 s$ hydrogen atoms, which then moved out of the interaction volume in a time short compared to the mean life of the atom. The radiation observed coming from the interaction volume was all due to radiative decay of the short-lived $2 p$ state.

A few months later Lichten and Schultz (1959) published a measurement of the $2 s$ excitation cross section, based on detection of free electrons ejected from a platinum surface on which the metastable atoms were incident. Other atomic hydrogen excitation measurements have appeared since that time. All are crossed beam measurements, based on improvements in high vacuum and electronic measurement technology.

The hydrogen atom crossed beam measurements do not suffer from the pressure dependent effects discussed 
in the previous section. The pressure is low enough in any of these beam experiments so that resonance radiation trapping and collisional transfer can be ignored. The problems of distortion arising from instrumental polarization cannot be ignored but they are easier to handle where broad band optical filters are used rather than prism or grating monochromators. On the other hand, there is a whole new set of problems. Signals to be detected are indeed minute. Interaction geometries are complicated. Electron optical problems may be more severe at the extremely low pressures required, since pump fluids and other contaminants migrate readily in a hard vacuum. Progress in the crossed-beam work has been difficult and time consuming. As a result only a few excitation measurements have appeared.

There remains a need for additional work to confirm the measurements now in the literature, and to extend the range of information available. There is also a need for more detailed and quantitative treatment of errors, so that the limitations of a given measurement are well defined. A measurement is complete only if the reliability and accuracy of the results are stated quantitatively. Systematic geometrical and physical errors should be identified and evaluated. The statistical errors of the measurement should be stated separately in well defined terms. The statement of a statistically determined probable error coupled with quantitative estimates of the magnitudes of systematic errors is valuable. A presentation of error bars two or three times the statistical probable error in the expectation that it covers a host of vaguely defined systematic effects provides little information.

There have been no accurate absolute measurements of hydrogen atom excitation cross sections. [The paper by Lichten and Schultz (1959) would seem to stand as a contradiction to this statement. The authors estimated $\sigma(2 s)=0.28 \pm 0.14 \pi a_{0}^{2}$ on the basis of a measurement of detector yields and other factors. However, in a note added in proof, they pointed to a possible error and referred to the Born approximation at approximately $40 \mathrm{~V}$ as the basis for an absolute scale. Thus the estimated accuracy of the absolute measurement is unspecified.] Normalization to the Born approximation is frequently used as the basis for quoting absolute values, and this sometimes obscures the fact that the measurements are relative. The major significance of the experimental excitation work has been to reveal the characteristics of cross sections in the lower energy range where the conventional approximations used in quantum theoretical calculations are generally found to be unreliable.

\subsection{Excitation of $B(2 p)$}

A schematic diagram of the apparatus developed by Fite and Brackmann (1958) for high vacuum crossed beam work is shown in Fig. 63. The technique utilizes a chopped atom beam crossed with a direct current beam of electrons in a field free region. An ionization chamber

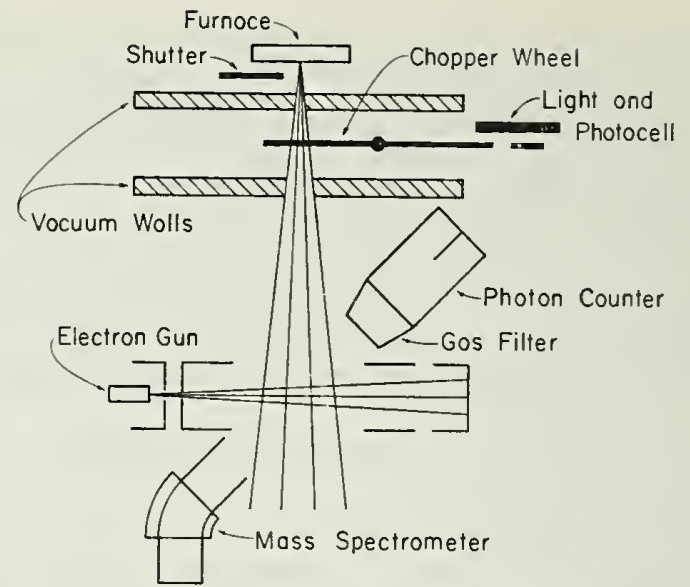

FIG. 63. Schematic diagram of the major elements of the crossed-beam apparatus used by Fite and Brackmann (1958) to study the excitation of Lyman- $\alpha$ radiation.

is used to detect the Lyman- $\alpha$ photons and the arrangement is such that the signal of interest must alternate synchronously with the beam chopping frequency. Microphonic signals as well as signals due to pressure modulation originating with the chopped beam may appear in addition to the signal due to Lyman- $\alpha$ photons originating from primary beam atoms. Care is required to guarantee the absence of such effects.

Another problem is the geometrical variation of the electron beam with energy. An excitation function can exhibit the correct energy dependence only if the integral of atom density over the electron trajectory is independent of electron energy. In a beam experiment it is necessary to ensure that all electrons pass through the full depth of the atom beam at all energies. It is also important to evaluate the effect on the integrated atom density traversed, of variations in the angular divergence of the electron beam. Further, consideration is needed of possible changes in photon collection and detection efficiency with such changes as may occur in the distribution of electron trajectories. If control of this geometry is not demonstrated in some way the value of the measurement is degraded. Structural details of the cross section might still be of interest but a comparison with theoretical shapes over a wide energy range would be of limited value. Other problems include atom beam purity, and the degree of spectral isolation of the radiation under investigation from radiation in other atomic and molecular lines which may be excited.

In Fig. 64 are shown excitation functions for the $2 p \rightarrow 1 s$ transition in atomic hydrogen, determined by observation of Lyman- $\alpha$ flux produced in a region free of electric fields, emitted in a direction perpendicular to the electron beam axis. The experimental results displayed include the results of the original measurement by Fite and Brackmann (1958), but exclude points near threshold now believed to be in error because they did not reproduce in subsequent careful measurements by Fite, Stebbings, and Brackmann (1959). The results of the latter set of authors have also been included, but 


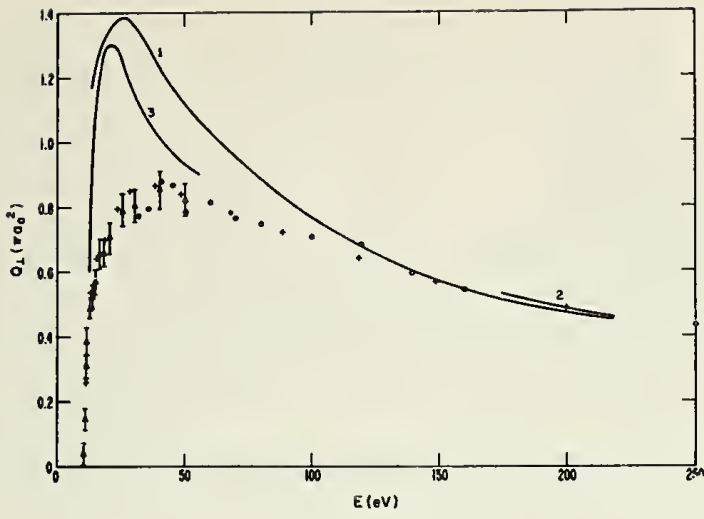

FIG. 64. Relative measurements of the cross section for excitation of the $2 p \rightarrow 1 s$ transition in atomic hydrogen [Fite and Brackmann (1958), O; Fite, Stebbings, and Brackmann (1959), $\triangle$; and Long, Cox, and Smith $(196 \%),+]$ compared with the Born approximation result for $Q_{\perp}$ (see text and Table XIII) without cascading (Curve 1) and with cascading (Curve 2), and compared with the result of a $1 s-2 s-2 p$ close coupling calculation for $Q_{\perp}$ (Burke, Schey, and Smith, 1963) (Curve 3) without cascading. Error bars shown for Fite et al. (1959) are confidence limits. Fite and Brackmann (1958) give $\pm 12 \%$ confidence limits. Long et al. (1967) results are limited by systematic errors of about $3 \%$.

we have renormalized them by a small factor to a better fit with recent results of Long, Cox, and Smith (1967).

Long, Cox, and Smith attempted to improve on the results of Fite et al. through a strenuous effort to reduce systematic and statistical errors to the order of a few percent at most. In the course of this work they found that the transmission of the lithium fluoride windows used on the Lyman- $\alpha$ detectors varied with electron energy, becoming more opaque at higher electron energies and with longer continuous exposure. The induced absorption decayed with a time constant of several seconds. Heath and Sacker (1966) had shown that lithium fluoride windows become opaque to Lyman- $\alpha$ when bombarded with $2-\mathrm{MeV}$ electrons. Heath and Sacker also found that magnesium fluoride windows are much less sensitive. Long, Cox, and Smith found that insertion of a magnesium fluoride window ahead of the detector eliminated the time-history-dependent effect, while insertion of another lithium fluoride window did not. The conclusion to be drawn is that the lithium fluoride window transmission is sensitive to $\mathrm{x}$ rays produced by electrons with energies of only about a hundred volts (no detailed study of the phenomena was carried out). These latter workers expected to obtain results different from the earlier results of Fite et al. when the $x$-ray effect was removed, but as shown in Fig. 64 the results are in complete agreement, within experimental errors, except for the small renormalization already mentioned. It appears that the effect was minimized but not identified in the work by Fite et al. We suggest that it may have been responsible for the original failure near threshold in the Fite and Brack- mann results, which has not otherwise been explained. Moderately high-energy electrons may have been present in the accelerating stages of their electron gun.

This confirmation of the observed $2 p \rightarrow 1 s$ excitation function permits a more definite conclusion that the theoretical methods described in Sec. 2.15 do not lead to an accurate description of the $2 p \rightarrow 1 s$ excitation function in the energy range below about 50 to $60 \mathrm{eV}$ except in the immediate vicinity of threshold. In Fig. 64 we have included the cross sections $Q \perp$ calculated using the Born approximation and the $1 s-2 s-2 p$ close coupling approximation [Burke, Schey, and Smith (1963)] together with the formula

$$
Q_{\perp}=0.918 Q_{2 p}+0.246 Q_{2 p_{0}}
$$

derived by Burke and Seaton (1960). The experimental results are normalized to the Born approximation, including a small allowance for cascading, at $200 \mathrm{eV}$. The measurements and the values calculated by the Born approximation seem to fit very well above about $120 \mathrm{eV}$, and it appears that the close coupling results may fit the measured values down to about $60 \mathrm{eV}$, a distinct improvement. However, below $50 \mathrm{eV}$ the departure of the close coupling results is quite striking.

This discrepancy leads to the speculation that the inclusion of the higher states in the close coupling scheme might reduce the cross section in this region. The calculations become extremely complex and expensive, and there is as yet no conclusive theoretical demonstration of what the effect of including coupling to all states might be. Burke (1963) showed that inclusion of close coupling to the $3 s$ and $3 p$ states reduced the value obtained for the total cross section at $16.5 \mathrm{eV}$ to 0.907 from the value of 1.094 obtained from a $1 s-2 s-2 p$ close coupling calculation. This is significantly higher than the experimental results on the basis of normalization to Born approximation at $200 \mathrm{eV}$.

Some intensive calculations have been carried out in the immediate vicinity of threshold by Burke, Ormonde, Taylor, and Whitaker (1967). These authors show that inclusion of close coupling to the $n=3$ level does not produce much of a change in the cross section at energies in the range from the $n=2$ threshold up to an energy somewhat below the $n=3$ threshold. However, it may be that at higher energies the cumulative effect of coupling to all the discrete levels with $n \geq 3$ and the continuum states would account for the difference between the $1 s-2 s-2 p$ close coupling approximation results and the observed behavior of the cross section.

Attempts to determine the polarization of Lyman- $\alpha$ radiation by comparing the excitation functions at two different angles (for example, $90^{\circ}$ and $45^{\circ}$ to the electron beam axis) have failed to yield reproducible and physically plausible results. The calculation of polarization from intensities at two angles involves the small differences in the intensities. Relative errors become very large and small systematic geometrical errors, 
such as result from the use of rather extended source volumes, have large effects.

Long, Cox, and Smith (1967) have concluded that the major difficulty derives from the sensitivity of lithium fluoride windows to low-energy $x$ rays. The window transmissions at a fixed position are found to vary with electron energy and with time of exposure. Furthermore, the behavior of the windows would vary with position since the exposures are dependent on position. Therefore, consistent measurements of polarization cannot be obtained using lithium fluoride-covered detectors.

A new technique, direct analysis of polarization by reflection at the Brewster angle from a sheet of lithium fluoride, seems promising, and preliminary results have been obtained by Ott, Kauppila, and Fite (1967).

Chamberlain, Smith, and Heddle (1964) studied the behavior of the $2 p$ excitation cross section in the immediate vicinity of threshold. Working with a carefully controlled electron beam geometry they obtained the experimental points for $Q_{\perp}$ shown in Fig. 65. The accuracy of these results depended on knowledge of the energy distribution of the electron beam. The energy distribution was obtained by analyzing the axial portion of the beam with a spherical retarding analyzer. The assumption was made that this energy distribution applied over the whole of the electron beam. Modification of beam energies by space charge fields was small with the $1.5-\mu \mathrm{A}$ beam used. Also shown in Fig. 65 is a curve based on recent threshold calculations for $Q_{\perp}$ by

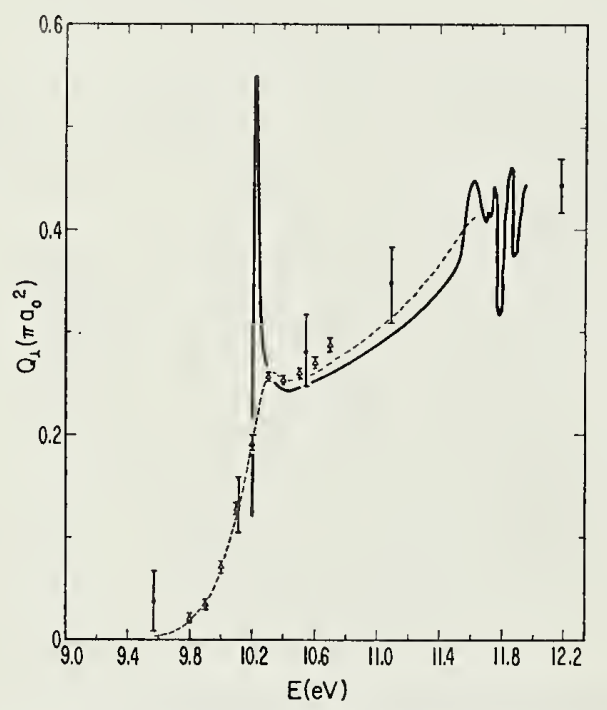

FIG. 65. Relative measurements of the cross section for excitation near threshold of the $2 p \rightarrow 1 s$ transition in atomic hydrogen [Chamberlain, Smith, and Heddle (1964), $\triangle$; and Fite, Stebbings, and Brackmann (1959), ○] compared with a composite (Burke, Taylor, and Ormonde, 1967) of a 3-state +20 correlation term calculation, below $11.4 \mathrm{eV}$, and a 6-state close coupling calculation, above $11.4 \mathrm{eV}$ (Burke, Taylor, Ormonde, and Whitaker, 1967). The dashed curve was obtained by folding the electron energy distribution from the work of Chamberlain et al. into the composite curve. Error bars for Fite $e t$ al. are confidence limits. Error bars for Chamberlain et al. are probable errors.

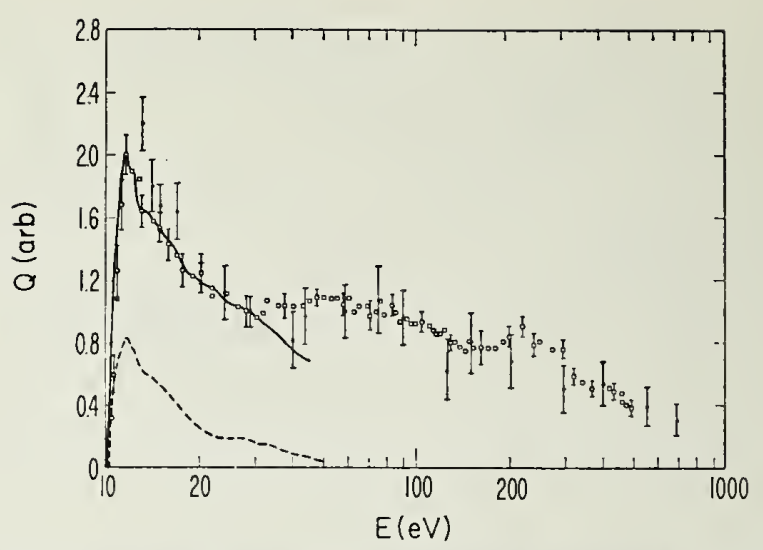

FIG. 66. Intercomparison of relative measurements for excitation of hydrogen atoms into the metastable $2 s$ state, including cascade contributions [Hils, Kleinpoppen, and Koschmieder (1966), $\square$; Lichten and Schultz (1959), solid line; Stebbings, Fite, Hummer, and Brackmann (1961) $\triangle$ ]. Hils et al. show error bars equal to twice rms errors plus $15 \%$ systematic errors. Stebbings et al. show confidence limits. Lichten and Schultz quoted a $15 \%$ error for the total cross section, the rms sum of all systematic errors and three times the probable statistical error. The dashed curve is the exchange cross section obtained by Lichten and Schultz, shown in the ratio to the total cross section determined by them to within $5 \%$.

Burke, Taylor, Ormonde, and Whitaker (1967). The dashed curve shows the result of folding the experimental energy distribution from Chamberlain et al. into the calculated resonant threshold. The experimental points have been scaled on the abscissa and shifted on the ordinate, this being allowable within reasonable limits since no accurate determination was made of the contact potentials involved. The resulting fit is quite good, noticeably better than can be achieved with a step function cross section or using the earlier results of Dambrug and Gailitis (1963).

Also shown in the figure are some of the points obtained by Fite, Stebbings, and Brackmann (1959) from their remeasurement of the threshold behavior. While their results do not reveal the threshold structure, they are consistent with the results of Chamberlain, Smith, and Heddle when account is taken of a larger energy distribution and larger error bars.

\subsection{Excitation of $H(2 s)$}

Three measurements of the excitation of the $2 s$ state of atomic hydrogen are shown in Fig. 66. We have renormalized the three available experimental results to facilitate direct comparison of the shapes. These are seen to be in fairly good agreement, considering experimental errors. Three aspects of the experimental results deserve particular notice: (1) the structure in the verylow-energy range best shown in the results by Lichten and Schultz (1959); (2) the gross structure through the medium-and high-energy range found by Hils, Kleinpoppen, and Koschmeider (1966) and which seems to be reflected in the work by Stebbings, Fite, Hummer, and Brackmann (1960, 1961); and (3) the over-all energy 
dependence of the measurements of Stebbings et al. and of Hils et al., which departs to a remarkable extent from that predicted by the Born approximation.

The Lichten and Schultz measurement, already briefly described, differed in basic principle from the other two measurements. The latter two relied on electric field quenching of the metastable atoms in free space within the field-of-view of a Lyman- $\alpha$ detector. In both cases the metastables were allowed to move out of the excitation region down the beam into a quench region.

The Stebbings, Fite, Hummer, and Brackmann measurement was a point-by-point comparison of the $2 s$ and $2 p$ excitation cross sections. The detector was mounted on a trolley so that the field-free excitation region and a down-stream region, in which a quenching field was applied, could be sequentially observed. The results were presented as a $2 s$ excitation cross section. The reliability of their result is dependent on the quality of the $2 p$ excitation cross section used, and this was not explicitly described.

The method of measurement by Hils, Kleinpoppen, and Koschmieder was similar to that used by Stebbings et al. An iodine counter with lithium fluoride window was used to detect the Lyman- $\alpha$ emitted from a region in which a quenching field was applied to the hydrogen beam. No chopper was used in this case, the application of the quenching field serving to differentiate between the signal and background.

The paper by Stebbings et al. describes in considerable detail the care taken to minimize errors due to the angular distribution of metastable atoms resulting from recoil, and due to spatial extent of the quenching field. They studied the angular distribution with which metastables came from the electron-beam-atom-beam intersection and found that the fraction of atoms with recoil angles much greater than $20^{\circ}$ was very small at all electron energies. Their apparatus was designed to subject all atoms within a $45^{\circ}$ angle to quenching within the field of view of the Lyman- $\alpha$ detector. The only comment we can add is to express the reservation that the field of view of the type of detector used is not homogeneous, and that a systematic error is to be expected from the variation with electron energy of the spatial distribution of metastables. At low energies the source volume would be more extended than at high electron energies.

It may also be noted that the soft x-ray mechanism for modifying lithium fluoride window transmission mentioned in the preceding section could possibly have been operative in the measurements of Stebbings et al., and of Hils et al. This effect is somewhat more likely in the former case because the measurement of $2 p$ excitation used for normalization involved direct observation of the region traversed by the electron beam. A depression of the $2 p$ signal due to induced opacity of the lithium fluoride could have led to a spuriously high relative value for $2 s$ excitation at higher energies.
Stebbings et al. do not present their primary data for the $2 p$ and $2 s$ excitation observed in this work.

The departure of the measured $2 s$ excitation cross sections from the Born approximation is so striking that normalization of experimental results to the theory at high energy is questionable. The usual procedure has been to normalize to the Born approximation plus a cascading correction at the highest energies for which measurements are available. The problem here is that the observed cross sections are so structured that one is reluctant to trust such a normalization. Hils et al. find a good fit over the range from 200 to $500 \mathrm{eV}$, but there is an abrupt departure from the Born result below 200 $\mathrm{eV}$ which does not occur for $2 p$ excitation.

Stebbings et al. found a good fit over the range from 400 to $700 \mathrm{eV}$. We would conclude from consideration of their error bars that a reasonable fit is possible from 150 to $700 \mathrm{eV}$, which results in a higher normalization, but this normalization is inconsistent with Hils et al. at lower energies. Stebbings et al. chose to normalize via the $2 p$ measurement to the Born approximation for $2 p$ excitation. The measured $2 p$ excitation fits the Born approximation to a much lower energy than is the case for $2 s$ excitation, and there is less uncertainty about the cascading correction for the $2 p$ cross section. On this basis, Stebbings et al. find their results for $2 s$ excitation in the 400 - to $700-\mathrm{eV}$ range lie about $50 \%$ higher than the Born cross section plus cascading. Their error bars fail to overlap the Born result at the three points in this range.

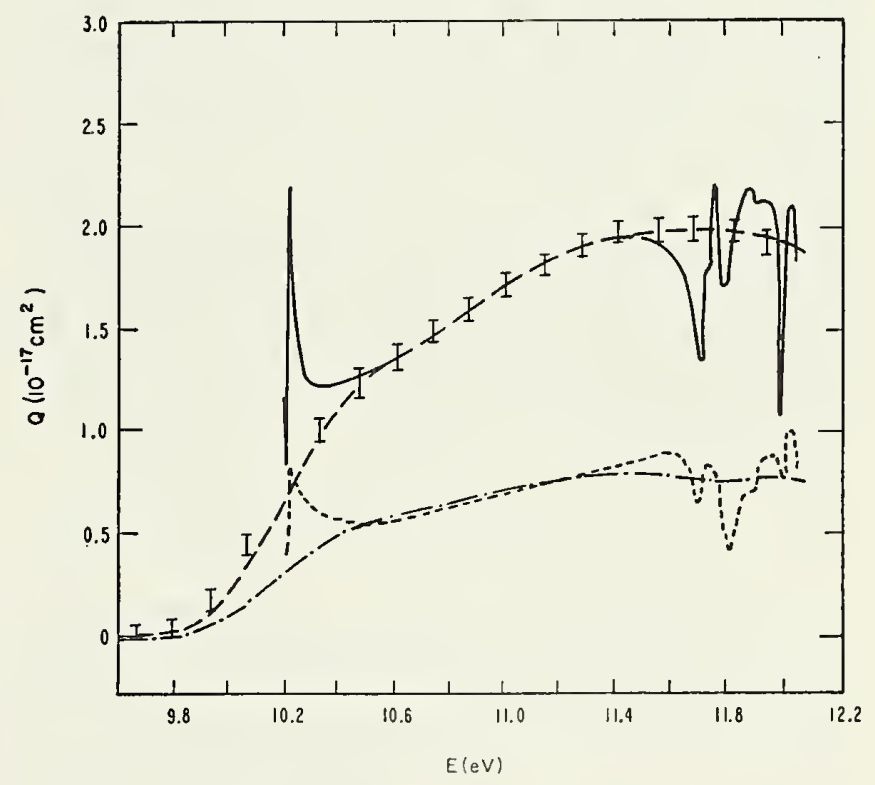

FrG. 67. The relative cross section near threshold for excitation of hydrogen atoms to the metastable $2 s$ state, measured by Lichten and Schultz (1959), represented by the bars, is compared with the results of close coupling calculations. The long dashed curve is the result of folding an electron-energy distribution of Gaussian shape and $0.2 \mathrm{eV}$ width into the theoretical curve. The lower set of curves are the corresponding results for the exchange cross section. The relative height of the two folded cross sections is consistent with the ratio determined by Lichten and Schultz. (Burke, Taylor, and Ormonde, 1967). 
Either of the above methods of normalization leads to a value for the peak near threshold between 0.1 and $0.15 \pi a_{0}^{2}$. In Fig. 67 we show the experimental results in the threshold region, due to Lichten and Shultz, in comparison with recent close coupling calculations by Burke, Taylor, Ormonde, and Whitaker (1967). It is the suggestion of Burke et al. that normalization to the peak at about $11.7 \mathrm{eV}$ may be valid. This is based on the observation that inclusion of the higher states has a small effect on the magnitude of the cross section at the peak, where the value is $\simeq 0.22 \pi a_{0}^{2}$. This normalization of the measurements leads to values at 500 to $700 \mathrm{eV}$ which are about twice as large as predicted by the Born approximation. This measured peak-to-high energy ratio is inconsistent with that predicted by the close coupling calculation, which presumably converges to the Born approximation at high energies.

In the case of $2 p$ excitation the measurements of the magnitude at threshold relative to the higher energy measurements, by S. J. Smith (1965), are consistent with the results of the close coupling calculation (Burke, Taylor, Ormonde, and Whitaker, 1967). The $2 s$ excitation cross section is in a relatively unsatisfactory state, and the theoretical and experimental results must both be suspect until the situation is clarified by further work.

\subsection{Excitation of Balmer Lines of Bydrogen}

Recent preliminary measurements by Kleinpoppen and Kraiss (1967) appear to have superseded the measurements of Ornstein and Lindeman (1933) for the Balmer- $\alpha$ line. The details of this relative measurement have not been published in full, but the principle difficulties lie in the necessity for differentiating the electron induced signal in the atom beam from the background of continuous radiation from the hydrogen disssociator. The Balmer- $\alpha$ excitation function shown in Fig. 68 includes contributions from populations in all angular momentum states of the $n=3$ level. The peak at threshold may be attributed to excitation of the $3 s$ and $3 d$ states while the broad maximum is mainly due to $3 p$ excitation.

Figure 69 shows the measured optical polarization of the Balmer- $\alpha$ lines excited by electron impact (Kleinpoppen and Kraiss, 1967). The fact that these observations include contributions from all available angular momentum states and that these states are sensitive to mixing by electric fields of magnitudes which may easily be produced by space-charge effects and field penetration, means that an interpretation of these observations in terms of excitation of the states of different orbital angular momenta cannot be quantitative.

\section{Excitation of Helium Positive Ions}

The excitation of $\mathrm{He}^{+}(1 s)$ differs from the excitation of atomic hydrogen in that the nuclear charge and mass are different. The principal physical difference is the influence of the long-range Coulomb field on the motion of incident electrons. The incident electron gains kinetic energy as it approaches the ion with the result that the $2 s \rightarrow 1 s$ excitation cross section is finite at threshold in the simplest Coulomb-Born approximation. For atomic hydrogen the finite threshold results from coupling to other states. Dance, Harrison, and Smith (1966) have measured the cross section for excitation of $\mathrm{He}^{+}$from the ground state $\mathrm{He}^{+}(1 s)$ to the metastable state $\mathrm{He}^{+}(2 s)$ using crossed charged beams in high vacuum. The experimental technique required was quite complicated because of the necessity for discriminating between the true excitation signal and several sources of background signal. Modulation techniques were used, but these were not capable of discriminating against the effects of space-charge modulation of one beam by the other. The correction for this effect was complicated by a dependence of the modulated ion beam geometry on the quenching field used for metastable detection.

The experimental results are shown in Fig. 70. Included in this figure is a trial cross section from the paper by Dance $e t$ al., chosen to give a good fit to the experimental points near threshold when folded with the electron energy distribution. The threshold points have been omitted from the figure for clarity. The experimental result is compared with the result of a close coupling calculation by Burke, McVicar, and Smith (1964a). There appears to be a serious discrepancy between the threshold behavior predicted by the close coupling calculations and that found experimentally.

\section{Helium}

Most of the existing helium excitation function data have been for transitions of the type $n(S, P, D) \rightarrow$ $2(S, P)$ ranging in wavelength from 2945 to $10829 \AA$. Transitions to the ground state are in the range from 500 to $600 \AA$, and are outside the range of conventional spectroscopy. Transitions terminating on levels of principle quantum number $n \geq 3$ are generally at wavelengths longer than $10000 \AA$, where photographic and photòelectric detection methods are insensitive. A few such lines are at wavelengths in the range 8000 to $10000 \AA$, but have not been studied, presumably because of low intensities due to comparatively small branching ratios. For the same reasons there are no observations of transitions corresponding to increasing orbital angular momentum, except, of course, those resulting from excitation of $S$ states. As a result there has been no more than one observed excitation function corresponding to each level of helium. It is understood that excitation functions refer to lines resulting from excitation of ground-state atoms.

\subsection{Helium $n^{1} P \rightarrow 2^{1} S$ Transitions}

Figure 71 presents relative measured apparent excitation functions for the $3^{1} P \rightarrow 2^{1} S$ transitions at $5016 \AA$, taken directly from the published tables or figures. All observations were made at $90^{\circ}$ to the electron beam axis. 


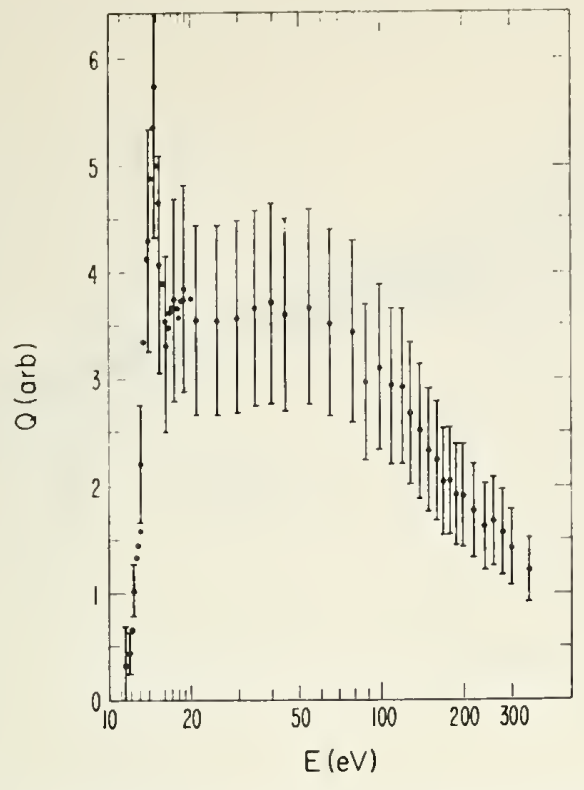

FIG. 68. Excitation function of the hydrogen Balmer- $\alpha$ line corrected for cascading. The errors are three times rms errors. (Kleinpoppen and Kraiss, 1967).

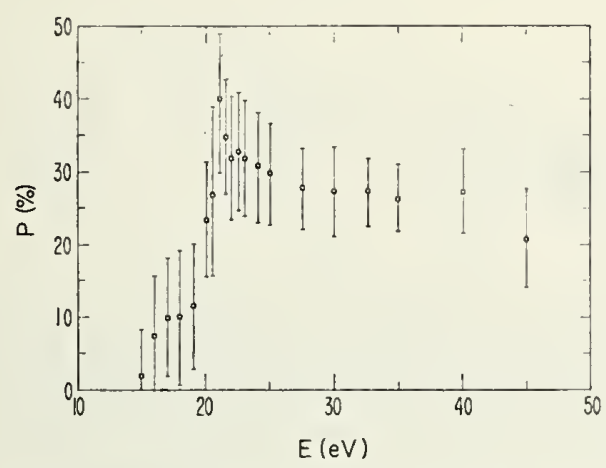

FIG. 69. Measured polarization of the hydrogen Balmer- $\alpha$ line as a function of electron energy. The errors are three times the rms errors plus $2 \%$ systematic errors. (Kleinpoppen and Kraiss, 1967).

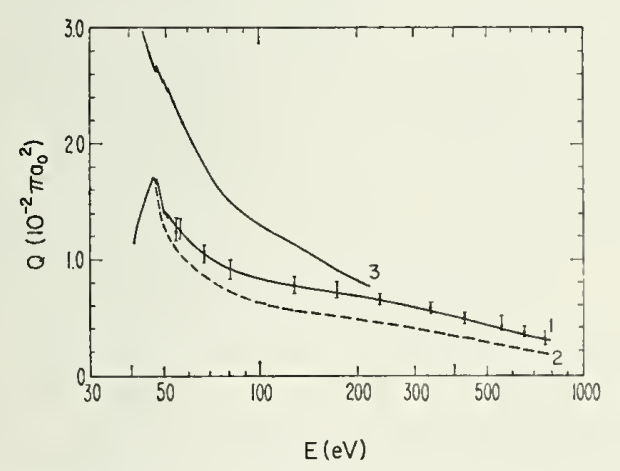

FIg. 70. Measured excitation function for $\mathrm{He}^{+}(2 s)$ from the $\mathrm{He}^{+}(1 s)$ ground state (Dance, Harrison, and Smith, 1966) compared with the result of a close coupling calculation by Burke, McVicar, and Smith (1964a) to which has been added an estimated cascade contribution (Curve 3 ). Curve 1 is a trial cross section chosen to give a good fit to the experimental points when folded with the electron energy distribution. Threshold points have been omitted from this figure. Curve 2 is derived by subtraction from Curve 1 of estimated cascading. The error bars represent $90 \%$ confidence limits.
One of the major factors contributing to the systematic differences among the $3^{1} P$ excitation functions shown in Fig. 71 is the polarization of the directly excited component of the $3{ }^{1} \mathrm{P} \rightarrow 2^{1} S$ line. The sources of such systematic differences have been discussed: distortion due to instrumental polarization, and pressure depolarization occurring to different degrees in the different measurements.

As has been mentioned, this line is extremely pressure sensitive due to imprisonment of radiation in the resonance line, $3^{1} P \rightarrow 1^{1} S$. This should not have a drastic effect on the shapes of the excitation functions. The major effect of imprisonment is an increase in the intensity of the $3^{1} P \rightarrow 2^{1} S$ line, due to effective modification of the lifetime of the $3^{1} P$ state and of the branching ratios. Some distortion of shapes due to imprisonment may occur through modification of cascading patterns, since transitions to the ground state are suppressed. Imprisonment of $n^{1} P \rightarrow 1^{1} S$ radiation, where $n \geq 5$, will increase the cascade contributions to the $3^{1} P$ population from higher $n^{1} P$ states through ${ }^{1} S$ and ${ }^{1} D$ states resulting in deformation of the $3^{1} P \rightarrow 2^{1} S$ excitation function near threshold. Away from threshold the distortion will be small because of the similarity of the $n^{1} P$ functions. Although collisional excitation transfer at the $n=3$ level may not be important, excitation transfer at higher levels may have some effect on the pattern of cascading into the $3^{1} P$ state. Figure 72 shows an estimate of the percentage cascade contribution to the $3^{1} P \rightarrow 2^{1} S$ line in the limit of low pressures. In this simplified calculation the $n^{1} P$ functions were assumed to have the same threshold for all $n$. Figure 72 also shows an estimate of cascade contribution at higher pressures where imprisonment is complete, but does not take into account the possible effects of excitation transfer.

We can estimate the extent to which the anisotropies in the radiation pattern may lead to the systematic differences in the excitation functions shown in Fig. 71. The measurements, carried out at different pressures, were subject to different degrees of pressure depolarization. Polarization and anisotropy are related in Eq. (148), which sirnplifies at $\theta=90^{\circ}$ to

$$
\langle I(\theta, E)\rangle_{\mathrm{Av}}=I\left(90^{\circ}, E\right)\{1-[P(E) / 300]\},
$$

where we have indicated dependence on energy $E$. Since polarization is energy-dependent, pressure depolarization causes a distortion in the shape of the apparent excitation function at $\theta=90^{\circ}$ from that which would be obtained at zero pressure.

A comparison of the various $3^{1} P \rightarrow 2^{1} S$ excitation functions which is more meaningful than that shown in Fig. 71 can be attempted by calculating $\langle I(\theta, E)\rangle_{\mathrm{Av}}$ for each of the measured excitation functions $I\left(90^{\circ}, E\right)$. This requires a knowledge of the polarization $P(E)$. For this purpose we have used the measurements of Heddle and Lucas shown in Fig. 73, where polarization measurements by several authors are compared. We have again referred to the results of Heddle and Lucas 


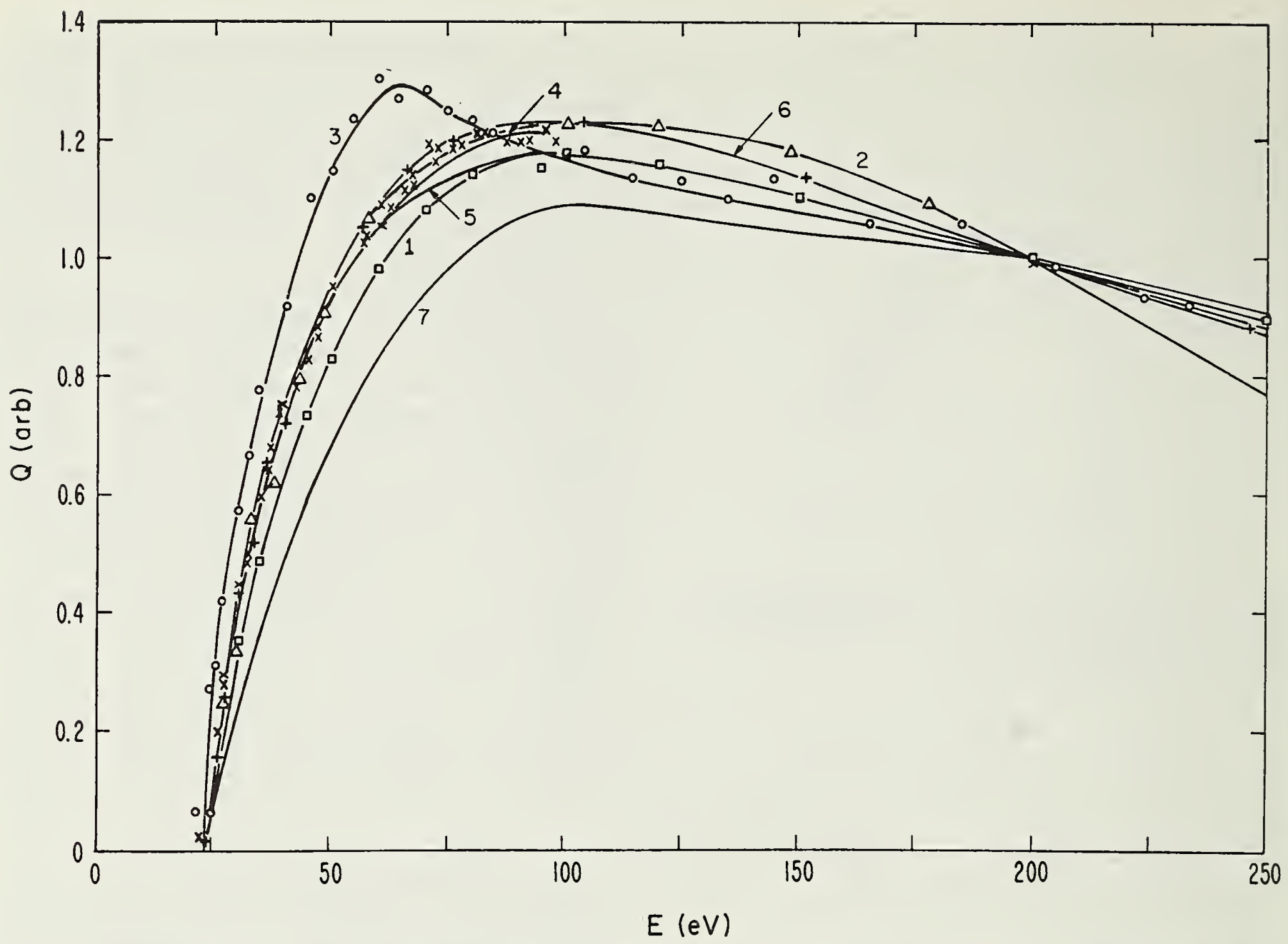

FIG. 71. Measured excitation functions for the $5016-\AA$ helium line $\left(3^{1} P \rightarrow 2^{1} S\right)$, normalized to unity at $200 \mathrm{eV}:$ St. John, Miller, and Lin (1964), $5.9 \mu$, Curve 1 and $\square$; Lees (1932), $44 \mu$, Curve 6 and + ; Thieme (1932), $5 \mu$, Curve 2 and $\triangle$; Heddle and Lucas (1963), $0.25 \mu$, Curve 3 and $O$; Yakhontova (1959), Curve 5; Zapesochnyi and Feltsan (1965), $\leq 0.04 \mu$, Curve 7; and Smit, Heideman, and Smit (1963), Curve 4 and $X$.

for the variation of polarization with pressure, and have applied an appropriate polarization reduction factor from Fig. 59 for each of the excitation functions. The resulting estimates of $I(E)$ are shown on a relative basis in Fig. 74.

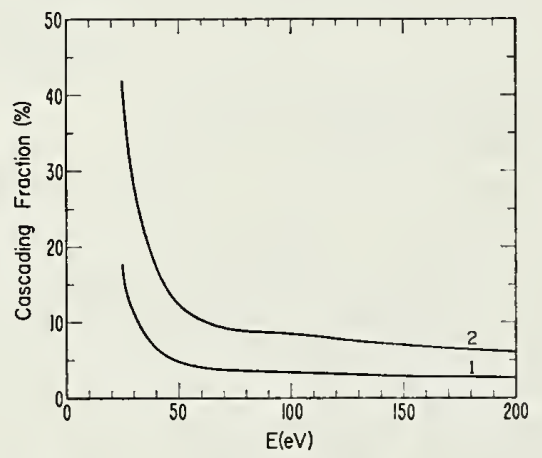

FIG. 72. The cascade fractions for the 5016- $\AA$ helium line $\left(3^{1} P \rightarrow 2^{1} S\right)$ are estimated for the optically thin (1) and optically thick (2) cases. Excitation functions from Heddle and Lucas (1963), absolute values from Gabriel and Heddle (1960), and helium transition probabilities from Wiese, Smith, and Glennon (1966) are used as the basis for the estimate. Excitation functions and cross sections are assumed to scale as $n^{-3}$, where $n$ is the principal quantum number, with no adjustment of threshold values. For the optically thick case, transitions to the ground state are suppressed.
The polarization corrections to the curves by Lees; Thieme; Yakhontova; St. John, Miller, and Lin; and Smit, Heideman, and Smit were small $(\sim 5 \%)$ since they were very largely depolarized at the pressures used. These pressures ranged from about 5 to $44 \mu$. It is particularly useful to intercompare these five curves. Since the radiation is almost completely depolarized, instrumental polarization errors should be small. Furthermore, as indicated earlier in this section, the effects of pressure on the shape of the $3^{1} P$ excitation function are not expected to be serious. The intercomparison of these five curves, then, should provide an indication of the scale on which other types of errors may occur.

The results of St. John et al. at low energies, and of Thieme at higher energies provide the largest departures from what would otherwise amount to a reasonable degree of consistency. For the St. John, Miller, and Lin curves in this and other figures of this section on helium, we have used tabular data published in Miller's thesis (1964). These data were taken from the continuous scan chart records of the excitation functions published at St. John et al. The quality of the chart records is not adequate to support a very accurate determination of threshold behavior for any of the helium excitation functions. The emphasis was on 

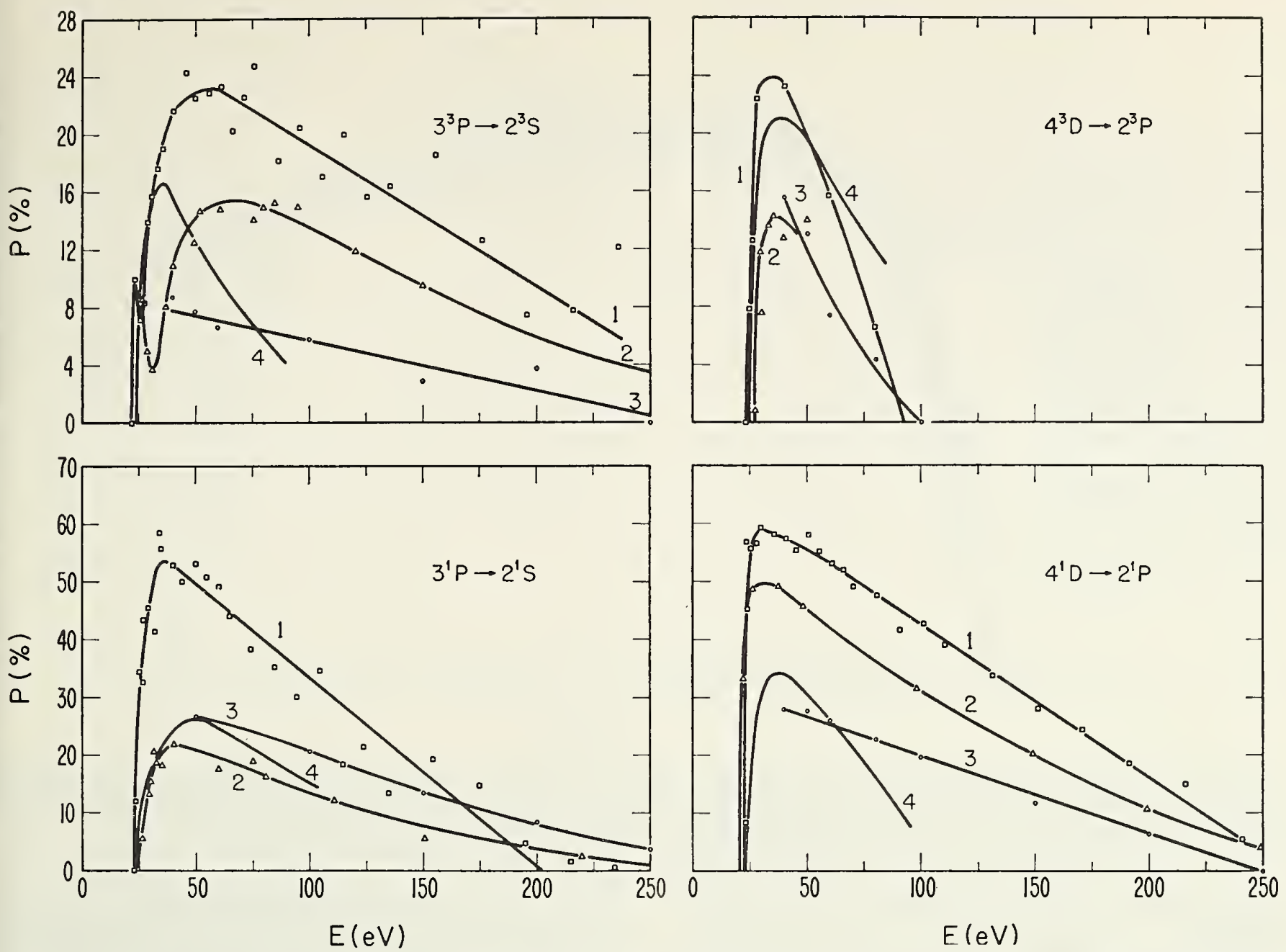

FIG. 73. The results of measurements of polarization of four helium lines excited by electron impact are intercompared [Heddle and Lucas (1963), Curves 1 and $\square$; McFarland and Soltysik (1962), Curves 2 and $\triangle$, except $4^{1} D$ which is McFarland (1967); Moustafa Moussa (1967), Curves 3 and O; and Dolgov (1959), Curves 4.]

behavior over a wider energy range $(0-500 \mathrm{eV})$. In fact, a detailed comparison of excitation functions for other helium lines, singlets, and triplets, shows that Miller's data is characterized by smaller initial slopes, and for the more resonant excitation functions, lower peaks shifted to higher energies than for the results of other workers, some of which were particularly concerned with threshold behavior. The shifts are so pronounced that they cannot easily be explained as due to electron energy distribution or space charge effects.

Thieme's excitation function differs from all the others in that his results fall off much more rapidly at the higher energies. Again this is characteristic of all Thieme's results for the singlet and triplet lines of helium. We have no explanation for this difference. However, the Thieme high-energy dependence stands alone against the fairly consistent results obtained by Yakhontova, Less, St. John, et al., and Heddle and Lucas. Furthermore, a recent experimental result by H. R. Moustafa Moussa (1967), primarily concerned with high energy dependence, exhibits a much smaller slope than Thieme's results and is much more nearly consistent with the results of the other authors just cited.

Moustafa Moussa also showed that the high-energy slopes of the ${ }^{1} P$ excitation functions are determined by the optical oscillator strengths, and that his own results for the ${ }^{1} P$ excitation functions are consistent with the theoretical predictions.

The Heddle and Lucas and the Zapesochnyi and Feltsan curves in Fig. 71 were obtained at such low pressures that the full polarization correction is required. While this correction brings the front end of the Heddle and Lucas curve fairly well into line in Fig. 74, the tendency of their curve to dip at medium energies can reasonably be assumed to result from inaccurate polarization measurements at these values. Heddle and Lucas summarized their polarization measurements by drawing a straight line going to zero at $200 \mathrm{eV}$. The scatter of the data is such that other choices are possible. The comparison of Fig. 74 suggests that their polarization values below $65 \mathrm{eV}$ are rather good, but that their curve should fall more rapidly at higher energies. In this medium energy region the data of McFarland and 


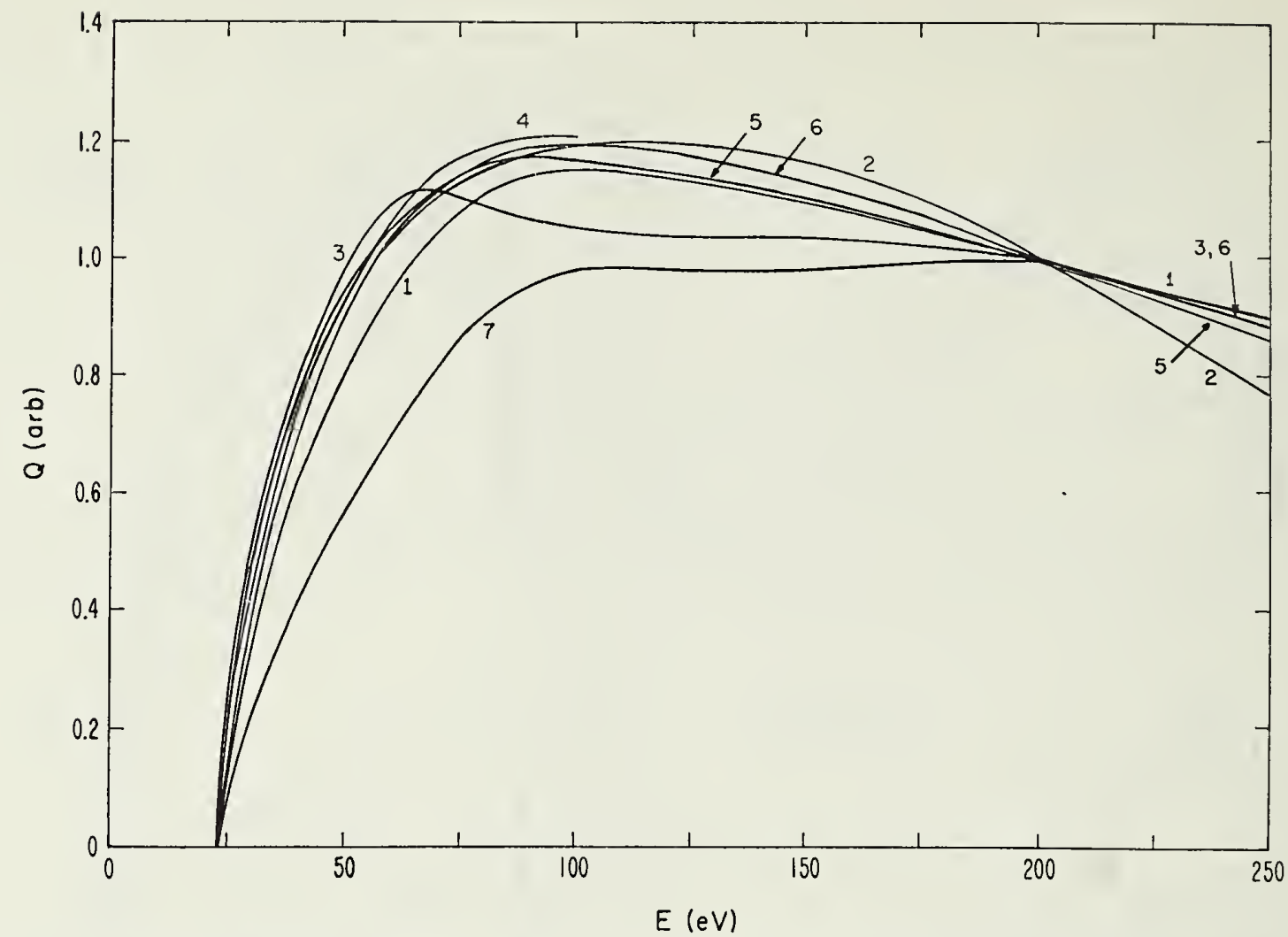

FIG. 74. The excitation functions shown in Fig. 71 are corrected for the polarization anisotropy using the polarization data and pressure depolarization data of Heddle and Lucas (1963) [St. John, Miller, and Lin, Curve 1; Thieme, Curve 2; Heddle and Lucas, Curve 3; Smit, Heideman, and Smit, Curve 4; Yakhontova, Curve 5; Lees, Curve 6; and Zapesochnyi and Feltsan, Curve 7].

Soltysik (1962) would have provided a more consistent correction.

Zapesochnyi and Feltsal,, who worked at the lowest pressures, obtained results which are in very poor agreement with all the others. The polarization correction makes the situation worse. This discrepancy cannot be definitely reconciled, but it appears possible that their monochromator, which according to their schematic diagram has a large number of oblique reflections and transmissions in the same plane, may have had a very high transmission asymmetry. This interpretation is consistent with the results for other helium lines; the results of Zapesochnyi and Feltsan are inconsistent with the results of other workers for the polarized lines, but are more nearly consistent for the unpolarized lines. It is also worth noting that according to Fig. 72 their result and that by Heddle and Lucas may include a smaller contribution from cascading than the other results, because of the low pressure at which they worked.

In Fig. 75 the excitation functions of Lees and Yakhontova, which we have corrected for cascading, have been compared with the energy dependence of the $3^{1} P$ excitation cross section calculated by Ochkur and Bratsev (1965b). Also included are results by H. R. Moustafa Moussa (1967), of measurements made at low pressure and published corrected for polarization and cascading.

The accurate absolute measurement of the magnitude of the $3^{1} P \rightarrow 2^{1} S$ excitation function is especially difficult because of the effect of imprisonment of resonance radiation. Figure 76 based on the work of Phelps (1958), is a graph showing high and low estimates of the effect of imprisonment of resonance radiation on the intensity of the $3^{1} P \rightarrow 2^{1} S$ line in helium. At zero pressure the fraction $f_{3-2}$ of $3^{1} P$ atoms giving rise to $3^{1} P \rightarrow 2^{1} S$ radiation is determined solely by the Einstein coefficients; i.e., $f_{3-2}=A_{3-2} /\left(A_{3-2}+A_{3-1}\right)=0.023$. In the very-low-pressure range the fraction $f_{3-2}$ in Fig. 76 can be represented approximately by a linear relation:

$$
f_{3-2}=0.023+0.04 \rho\left(p \times 10^{3}\right),
$$

where $\rho$ is the radius in centimeters of the cylindrical reaction chamber and $p$ is the helium pressure in Torr. Helium pressures in the vicinity of a few times $10^{-5}$ Torr evidently are required to obtain absolute values for the $3^{1} P \rightarrow 2^{1} S$ excitation function which approach to within a few percent of the zero pressure value.

Equation (176) may be difficult to apply to obtain corrections in the low-energy range because of the question of definition of the effective radius $\rho$ of the collision chamber in an apparatus for which only a fraction of the diameter of the reaction chamber is focussed onto the monochromator entrance slit. The pressure dependence of an excitation function would have to be determined experimentally to permit an exact correction or to determine the safe pressure range corresponding to some maximum error. Most of the experimental papers men- 


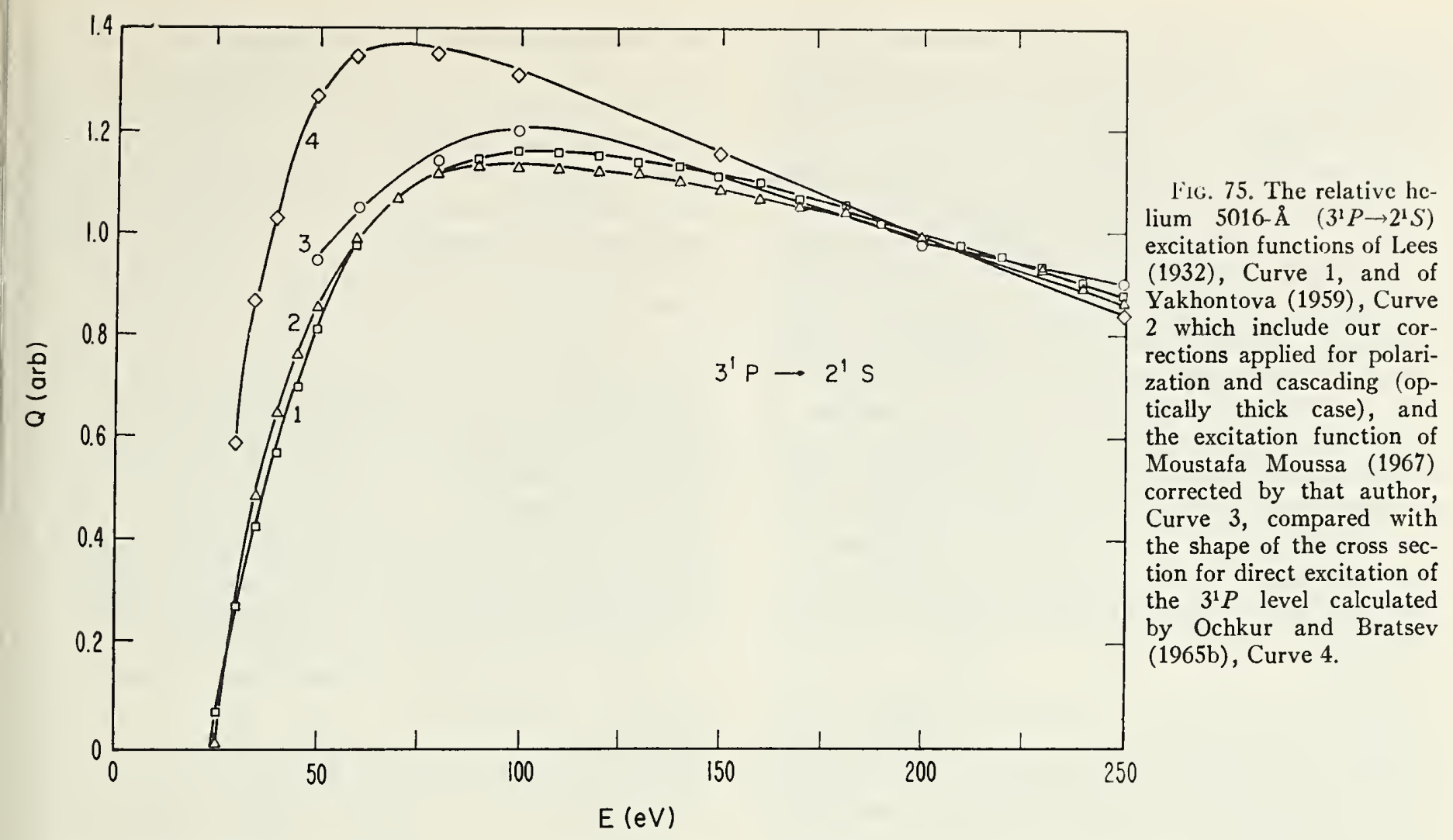

tioned below include linearity checks of one form or another. However, in every case the linearity data contains scatter much larger than a few percent in the $10^{-5}$ to $10^{-4}$ Torr range. It must be presumed that all of the absolute measurements could have been seriously affected by imprisonment of resonance radiation.

Measurements of the absolute value of the $3^{1} P \rightarrow 2^{1} S$ excitation function at very low pressures were accomplished by St. John, Bronco, and Fowler (1960), and later by St. John, Miller, and Lin (1964). The latter published a peak value (at $\simeq 100 \mathrm{eV}$ ) for the apparent excitation cross section of the $3^{1} P$ level of $350 \times 10^{-20}$ $\mathrm{cm}^{2}$ per atom. This value was obtained by absolute measurement of the intensity in the $3^{1} P \rightarrow 2^{1} S$ line "at pressures as low as $10^{-4}$ Torr." Transition probabilities calculated by Dalgarno, Lynn, and Stewart [from tables published by Gabriel and Heddle (1960) who referenced these to Dalgarno, Lynn, and Stewart (1959, private communication)] were used to calculate the apparent excitation cross section for the $3^{1} P$ level, on the assumption that imprisonment of resonance radiation is negligible at these pressures. A curve of pressure dependence of the excitation is given in Miller's thesis but the data shown in the low-pressure range are not sufficient to support that assumption. Equation (176) suggests that imprisonment may have been responsible for 10 or $20 \%$ of the observed intensity. No estimate of the experimental error in the absolute measurement of the excitation function is given.

This "apparent" excitation cross section is uncorrected for polarization and cascading effects. The au- thors did not measure the polarization of the lines observed in their measurements. To obtain a correction they applied Eq. (158) using the data of McFarland and Soltysik (1962). Their correction was $-4.4 \%$ at $100 \mathrm{eV}$. An additional correction for cascading of 4 percent at $100 \mathrm{eV}$ then led to a $3^{1} P$ level-excitation cross section of $\sim 322 \times 10^{-20} \mathrm{~cm}^{2}$ per atom.

Another low-pressure measurement was carried out by Zapesochnyi and Feltsan (1965). They worked at pressures $\leq 4 \times 10^{-5}$ Torr, where imprisonment effects could be of the order of a very few percent. They give a

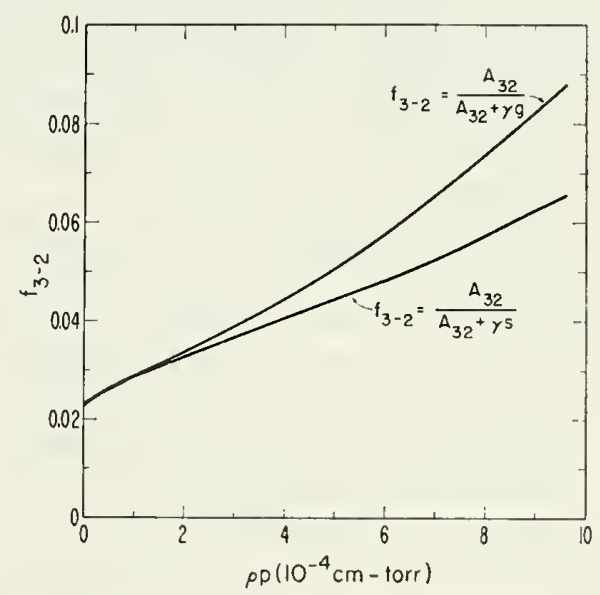

FIG. 76. Estimates of the effect of imprisonment of resonance radiation on the fraction $f_{3-2}$ of helium atoms excited to the $3^{1} P$ state which contribute to observed $5016-\AA$ emission. The two curves are based on imprisonment coefficients $g_{R}$ and $s_{R}$ given by Phelps (1958) in his Fig. 4. The pressure $p$ is in Torr and the effective radius of the interaction chamber $\rho$ is in centimeters. 
peak value of $17.4 \times 10^{-20} \mathrm{~cm}^{2}$ for the absolute apparent excitation function of the $3^{1} P \rightarrow 2^{1} S$ line. This corresponds to a cross section for excitation of the $3^{1} P$ level of about $750 \times 10^{-20} \mathrm{~cm}^{2}$. They estimated the error as being $50-100 \%$ from all causes. Corrected for cascading and polarization, this becomes $675 \times 10^{-20} \mathrm{~cm}^{2}$.

V. E. Yakhontova (1959) measured the absolute value of the excitation cross section for the $3^{1} P$ level by working at a pressure of 0.1 Torr where imprisonment of resonance radiation is essentially complete so that all excitations to the $3^{1} P$ level give rise to $3^{1} P \rightarrow 2^{1} S$ radiation. Under these circumstances no polarization correction is needed since pressure depolarization is essentially complete. A magnitude of $3.5 \times 10^{-20} \mathrm{~cm}^{2}$ at 100 $\mathrm{eV}$ was obtained for the excitation of the line, uncorrected for cascading. This magnitude corresponds to a cross section for excitation of the $3^{1} P$ level of about $150 \times 10^{-20} \mathrm{~cm}^{2}$. The author refers to an auxiliary investigation of secondary processes as indicating that these are small.

Gabriel and Heddle (1960) measured the $3^{1} P \rightarrow 2^{1} S$ excitation function over a range of pressures using photographic detection. By applying Phelps' trapping analysis to data taken at $0.011,0.03,0.06$, and 0.1 Torr they obtained a value for the $3^{1} P$ level-excitation cross section of $457 \times 10^{-20} \mathrm{~cm}^{2}$, at $108 \mathrm{eV}$, corrected for cascading. The radiation is so nearly depolarized at these pressures that correction for angular anisotropy is negligible. The method would seem to be subject to considerable uncertainty since there are several free parameters; e.g., the effective radius $(\sim 0.5 \mathrm{~cm})$ and the collisional transfer cross sections as well as the excitation cross section are simultaneously determined from the four measurements.

Moustafa Moussa (1967), working at Leyden, has published absolute excitation cross sections for a number of helium lines over the energy range from $50 \mathrm{eV}$ to 6 $\mathrm{keV}$. For the $3^{1} P$ cross section at $10^{-4}$ Torr he obtained $260 \times 10^{-20} \mathrm{~cm}^{2}$ at $100 \mathrm{eV}$ after correction for polarization $(7 \%)$ and cascading $(4.1 \%)$. The pressure is high enough, according to Fig. 76, so that a significant imprisonment error could have occurred.

Other absolute measurements, by Lees (1932), by Thieme (1932), and by Stewart and Gabathuler (1959) were carried out at pressures for which imprisonment of the resonance line is severe. Phelps has shown that the results of Lees and of Thieme are consistent with the theory of imprisonment of resonance radiation if an effective radius of $0.75 \mathrm{~cm}$ is assumed for their somewhat similar excitation chambers.

The range of results, from 150 to $750 \times 10^{-20} \mathrm{~cm}^{2}$ for the $3^{1} P$ level excitation cross section, certainly is unsatisfactory. If it were necessary to narrow the choice we would disregard the results obtained by Yakhontova because of the errors introduced by excitation transfer at the high pressures used. The remaining results differ within a factor of three. Zapesochnyi and Feltsan admit to the possibility of their result being 1.5-2 times too large so that the two results are apparently not contradictory, but by inference they are allowing for trapping of resonance radiation. They worked at lower pressures than did St. John, Miller, and Lin. Obviously there were gross inaccuracies in several of these measurements. It is impossible to make a valid judgment as to where the correct answer might lie. These absolute values and those for the higher $n^{1} P$ excitations are listed in Table $\operatorname{LXIV(a),~as~are~absolute~values~calcu-~}$ lated at $100 \mathrm{eV}$ by Ochkur and Bratsev (1965b). It is interesting to note that there is rather good agreement in the measured ratios of the $3^{1} P$ and $4^{1} P$ excitation cross sections at 5016 and $3965 \AA$. The work at a given laboratory tends to be internally consistent, but the basic radiometric, pressure, and other calibrations are not consistent from one laboratory to another. Improvement of radiometric and density standards is needed.

In Table LXIV a-f we have applied branching ratios to convert the photon-excitation cross sections to cross sections for populating the upper levels. Zapesochnyi (1966), in Astron. Zh., has summarized results obtained in his laboratory over several prior years. His results are presented as direct excitation cross sections for the levels corrected for cascading. Because we have not included cascade corrections the values we have obtained from Zapesochnyi and Feltsan (1965) do not agree for all levels with the values presented by Zapesochnyi (1966). A level by level comparison suggests that Zapesochnyi may have introduced corrections other than for cascading or may have included some results not otherwise published. For example, Zapesochnyi (1966) lists the peak $3^{1} P$ level excitation cross section as $530 \times 10^{-20} \mathrm{~cm}^{2}$. In the text above we have given a value of $675 \times 10^{-20} \mathrm{~cm}^{2}$ based on Zapesochnyi and Feltsan (1965). Since the source of Zapesochnyi's numbers is obscure we have not included them in our table.

Zapesochnyi found that his peak level-excitation cross sections for a given series lie along a straight line on a $\log -\log$ plot against principle quantum number. The peak cross sections for each series of levels can be represented by the expression

$$
Q_{L}(\max )=c / n^{\alpha},
$$

where $c$ and $\alpha$ are constants for each value of orbital angular momentum $L$, and $n$ is the principal quantum number. He finds $\alpha=3$ for the ${ }^{1} P,{ }^{1} D$, and ${ }^{3} D$ levels, $\alpha=4$ for the ${ }^{1} S$ and ${ }^{3} S$ levels and $\alpha=9$ for the ${ }^{3} P$ levels. Values for the $2^{1} S, 2^{1} P$, and $2^{3} S$ cross sections are obtained by extrapolation. No physical justification is given for formula (177).

The same general physical considerations apply to excitation of the higher $n^{1} P$ levels as apply to the $3^{1} P$ level, with the exception that collisional transfer of excitation may be more severe for the higher levels. In particular, imprisonment of resonance radiation presents essentially the same problem for each of the 


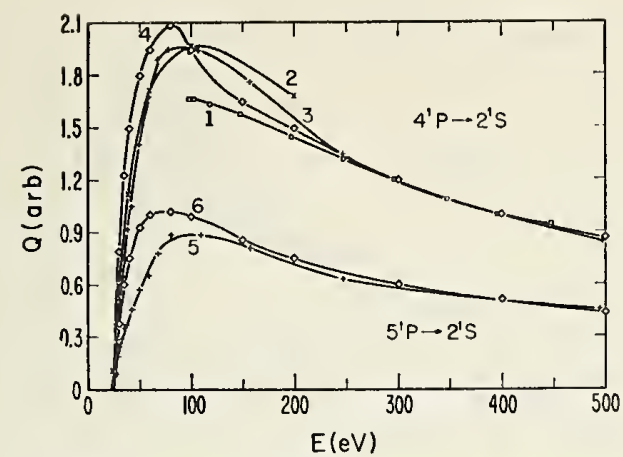

Fig. 77. Relative excitation functions for the helium $4^{1} P$ and $5^{1} P \rightarrow 2^{1} S$ transitions [St. John, Miller, and Lin (1964), $2 \mu$, Curve 1; Yakhontova (1959), Curve 2; Lees (1932), $44 \mu$, Curves 3 and 5] compared with direct excitation cross section for $4^{1} P$ and $5^{1} P$ levels calculated by Ochkur and Bratsev (1966), Curves 4 and 6 . The experimental data are uncorrected for polarization or cascading. The Ochkur and Bratsev $4^{1} P$ and $5^{1} P$ cross sections are shown with the proper relative magnitudes. The data obtained by Moustafa Moussa (1967) for $4^{1} P$ excitation closely correspond to Curve 4 above $100 \mathrm{eV}$, falling below it at lower energies; and for $5^{1} P$ they fit Curve 6 at all energies.

lines $n^{1} P \rightarrow 2^{1} S$. In the low-pressure range the fraction $f_{n-2}$ of $n^{1} P$ atoms which give rise to $n^{1} P \rightarrow 2^{1} S$ radiation can be represented by

$$
f_{n-2}=B_{n-2}+\left(0.04 A_{n-1} / A_{3-1}\right) \rho\left(p \times 10^{3}\right),
$$

where $A_{n-m}$ is the transition probability for the line $n^{1} P \rightarrow m^{1} S$. The first term on the right, $B_{n-2}$, is the branching ratio for the $n^{1} P \rightarrow 2^{1} S$ line, which does not vary greatly within this series. The pressure-dependent term is approximately proportional to the transition probability of the appropriate resonance line, and decreases by a factor of two or less with each higher level. The pressure $p$ is in Torr and the effective radius $\rho$ is in centimeters.

All these $n^{1} P$ lines are $100 \%$ polarized at threshold according to Percival and Seaton, and presumably behave qualitatively in the same way as the $3^{1} P \rightarrow 2^{1} S$ line with respect to energy dependence and pressure dependence of the polarization.

Some experimentally determined relative excitation functions for the $4^{1} P \rightarrow 2^{1} S$ and $5^{1} P \rightarrow 2^{1} S$ lines are shown in Fig. 77. Many of these results were obtained at pressures high enough so that pressure depolarization and imprisonment of resonance radiation are very effective. Zapesochnyi and Feltsan (1965) carried out measurements of excitation functions for the $4^{1} P, 5^{1} P$ and $6^{1} P \rightarrow 2^{1} S$ lines, not included in the figures, which have much the same characteristics as their $3^{1} P \rightarrow 2^{1} S$ function shown in Fig. 71. Thieme's data for $4^{1} P$ excitation is also omitted. They are characterized by a steep slope at the higher energies.

Moustafa Moussa used an ultraviolet spectrometer to obtain the first measurements of the $2^{1} P$ excitation cross section. He measured the energy dependence but did not obtain an absolute value.

\subsection{Helium $n^{1} S \rightarrow 2^{1} P$ Transitions}

The situation for the $n^{1} S \rightarrow 2^{1} P$ series of excitation functions is usually presumed to be simpler than for $n^{1} P$ excitation. The $n^{1} S$ state has spherical symmetry and the lines are not polarized so that there is no question of instrumental polarization effects or of angular distribution. Furthermore, since the $n^{1} S$ state does not optically connect to the ground state the problem of imprisonment of resonance radiation enters only through the population contributed by cascading from the ${ }^{1} P$ levels. Therefore, the poor agreement between the available measurements has been regarded as surprising. However, because of the cascading contribution these lines are much more pressure sensitive than the corresponding triplet series, for example. Figure 78 shows estimates of the cascade contribution to the $4^{1} S \rightarrow 2^{1} P$ line at several pressures. The extreme sensitivity of the cascading fraction to pressure in the range where most of the measurements were carried out has not been recognized.

The available measured excitation functions are clearly of limited value, in view of this pressure dependence. Figure 79 displays the uncorrected data for the 5048- $\AA \quad\left(4^{1} S \rightarrow 2^{1} P\right)$. line. Here we have normalized the experimental results at $60 \mathrm{eV}$ and shown them in comparison with the shape of the theoretical curve by Fox (1965). It should be noted that the cascading fraction is quite significant at $1-\mu$ pressure and varies sharply with pressure. Furthermore, the pressures are not explicitly stated for two of the curves. The comparison with theory should not be carried too far here.

Data for the $4438-\AA\left(5^{1} S \rightarrow 2^{1} P\right)$ excitation function is displayed in Fig. 80. The comparison with theory looks much the same as for the $4^{1} S \rightarrow 2^{1} P$ excitation function and should be regarded as only qualitative since the measurements were carried out at pressures for which cascading should be large.

A few results are available for the $3^{1} S$ (St. John et al., Zapesochnyi and Feltsan, and Moustafa Moussa),

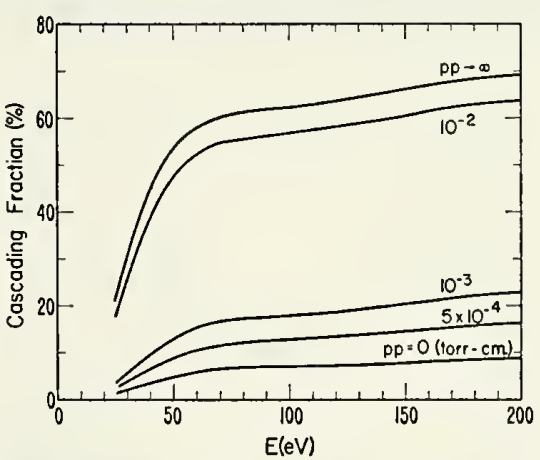

Fig. 78. The cascade fractions for the helium $5048-\AA$ line $\left(4^{1} S \rightarrow 2^{1} P\right)$ are estimated for several values of the product $p \rho$, where $p$ is pressure in Torr and $\rho$ is effective radius of the collision volume. The basis of the calculations is the same as for Fig. 72 . In addition the imprisonment curves of Fig. 76 and data from the text are used to estimate the degree of suppression of transitions to the ground state from higher ${ }^{1} P$ states. 
FIG. 79. Relative excitation functions for the helium $4^{1} S \rightarrow 2^{1} P$ transition [St. John, Miller, and Lin (1964), $1.5 \mu$, Curve 1; Heddle and Lucas (1963), $1.2 \mu$, Curve 2; Thieme (1932), Curve 3; Yakhontova (1959), Curve 4; and Zapesochnyi and Feltsan (1965), Curve 5] are compared with the $4^{1} S$ levelexcitation cross section calculated by Fox (1965), Curve 6.

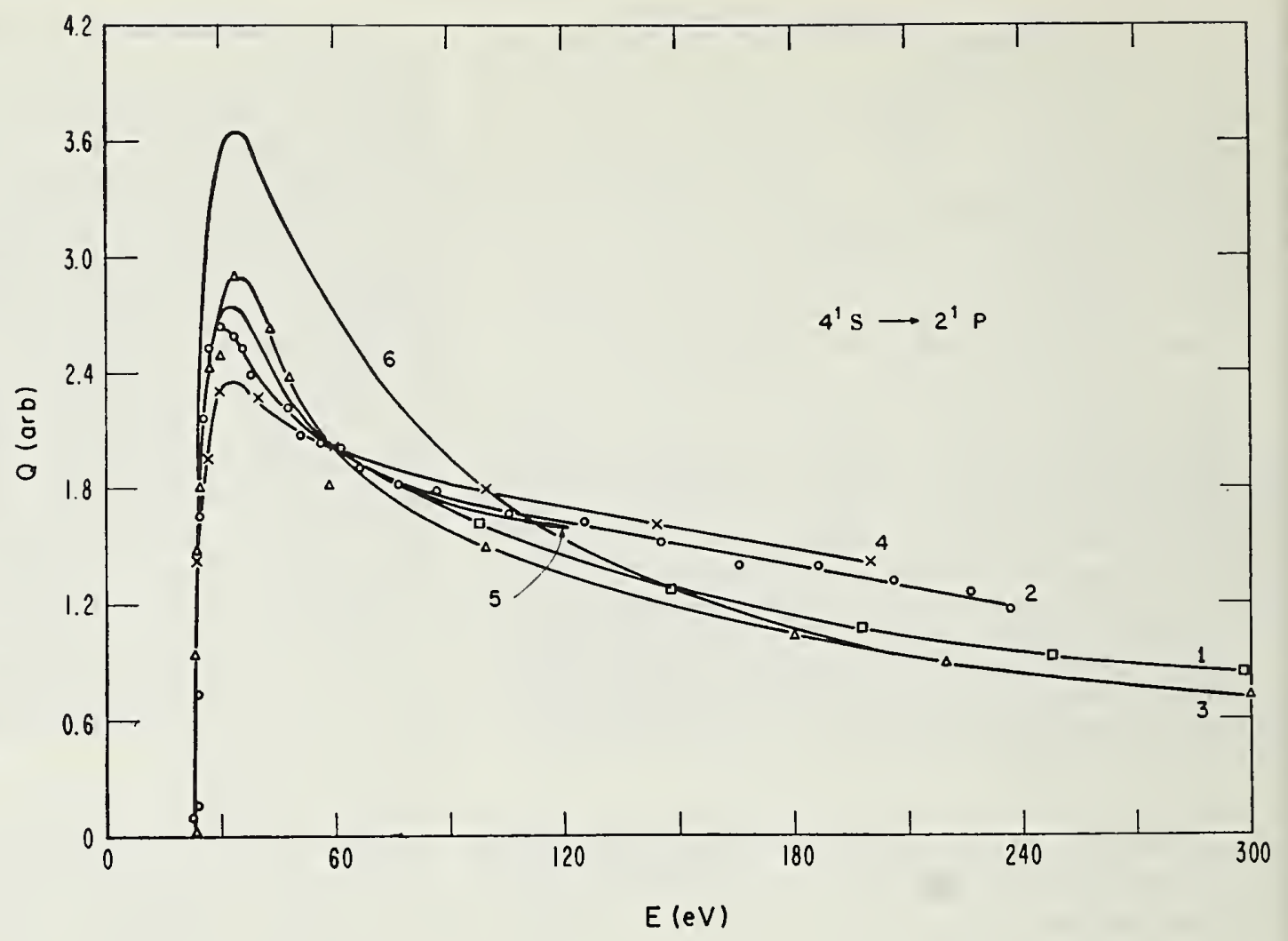

$6^{1} S$ (Thieme, St. John et al., Zapesochnyi and Feltsan, and Moustafa Moussa), and $7^{1} S$ (Zapesochnyi and Feltsan) excitation functions. These results are similar to the results for the $4^{1} S$ and $5^{1} S$ functions. The Zapesochnyi and Feltsan results are limited to the energy range below $40 \mathrm{eV}$.

Measurements of a large number of helium excitation functions by $\mathrm{H}$. R. Moustafa Moussa became available too recently to be considered in planning the figures for this review article. We have added them where possible to the already drafted figures. Moustafa Moussa's data extends from $50 \mathrm{eV}$ to about $6 \mathrm{keV}$. Our figures were designed to emphasize the lower energy range, and extend typically to $250 \mathrm{eV}$ so that the slope and curvature of excitation functions are displayed at energies

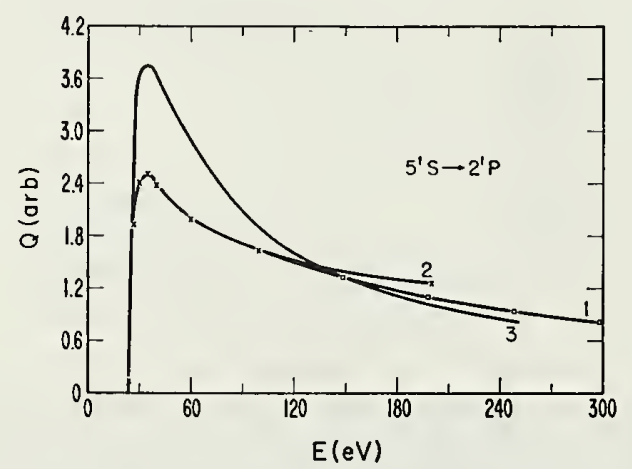

FIG. 80. Relative excitation functions for the $5^{1} S \rightarrow 2^{1} P$ helium transition [St. John, Miller, and Lin (1964), $5.5 \mu$, Curve 1; and Yakhontova (1959), Curve 2] are compared with the $5^{1} S$ level excitation cross section calculated by Fox (1965), Curve 3. higher than the energy of maximum excitation. Moustafa Moussa's data for the ${ }^{1} S,{ }^{1} D,{ }^{3} S,{ }^{3} P$, and ${ }^{3} D$ excitation functions do not contain information about the shape of the peak and threshold regions, but show the shape beyond the peak. In comparing Moustafa Moussa's data with other results we have used data given in his thesis to reintroduce cascading and polarization effects.

For $4^{1} S$ excitation the negative slope of the Moustafa Moussa curve is slightly less than that of the curve representing the work of St. John, Miller, and Lin. The two would be indistinguishable in our Fig. 79. For $5^{1} S$ excitation, shown in Fig. 80, Moustafa Moussa's results again have a slightly smaller negative slope in the range from 75 to $250 \mathrm{eV}$ than that of the results by St. John et al.

In Table LXIVb are listed the absolute values of the $n^{1} S$ levels measured by a number of workers. Most of these absolute values are measured at the peak of the excitation function. This practice of measuring absolute values at the peaks may not be the best in every case, particularly for the triplet lines because of the very sharp peaking near threshold. In the first place, variations in the widths of electron energy distribution from one measurement to another may well make comparisons between peak values rather meaningless. In the second place the entire question of polarization near tions to peak values for angular distribution and instrumental effects may not be meaningful. In the third place, the question of pressure sensitivity near threshthreshold seems to be quite undecided so that correc- 


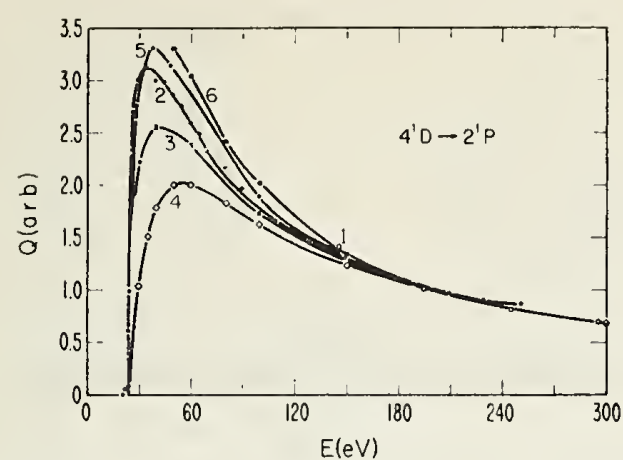

FIG. 81. Relative excitation functions for the helium $4^{1} D \rightarrow 2^{1} P$ transition [St. John, Miller, and Lin (1964), $1.7 \mu$, Curve 1; Heddle and Lucas (1963), $1.2 \mu$, Curve 2; Yakhontova (1959), Curve 3; McFarland (1967), $10^{-2} \mu$ (crossed-beam measurement), Curve 5; and Moustafa Moussa (1967), $\sim 1 \mu$, Curve 6] compared with the $4^{1} D$ excitation cross section calculated by Ochkur and Bratsev (1965), Curve 4.

old, raised by Bogdanova and Geitsi, leads to some uncertainty about the validity of peak measurements. We have extrapolated the $n^{1} S$ absolute measurements to $100 \mathrm{eV}$, and compared them in Table LXIVb with the absolute values calculated by Fox.

\subsection{Helium $n^{1} D \rightarrow 2^{1} P$ Transitions}

The ${ }^{1} D$ excitation process results in radiation which is rather strongly polarized (see Fig. 73). Therefore, all the earlier discussion of distortion due to polarization is applicable here. The possibility of Larmor precession causing depolarization is especially significant here because of the longer lifetimes involved (see Figs. 61 and 62). Also, as discussed by Lin and St. John (1964), these excitation functions are subject to distortion by excitation transfer, presumably from the high $n^{1} P$ states to $n^{1} F$ states followed by cascading. The pressure dependence curve for $4^{1} D \rightarrow 2^{1} P$ radiation of Heddle and Lucas (1963) does not indicate a very great sensitivity to pressure, but it was made at $35 \mathrm{eV}$, well below the peak of the $n^{1} P$ cross sections. The distortion should be greatest at $100 \mathrm{eV}$ where the $n^{1} P$ cross sections have their maxima.

We have not calculated the cascading fraction and its pressure dependence for lack of reliable parameters and quantitative information about excitation transfer at higher levels. The ${ }^{1} D$ excitation functions are of the same order of magnitude as the ${ }^{1} S$ functions, while the $n^{1} P \rightarrow m^{1} D$ transition probabilities are typically a third or a fourth of the corresponding $n^{1} P \rightarrow m^{1} S$ transition probabilities. Therefore, the $n^{1} P \rightarrow m^{1} D$ cascading is relatively smaller than the $n^{1} P \rightarrow m^{1} S$ cascading. Cascading fractions for ${ }^{1} D$ excitation functions should be smaller than for ${ }^{1} S$ excitation functions. Cascading also occurs from ${ }^{1} F$ states but St. John et al. estimate the cascading from ${ }^{1} F$ levels to be about a tenth of the cascading from ${ }^{1} P$ levels. They give the total cascading fraction as a maximum percentage ( 5 to $8 \%$ ) at $450 \mathrm{eV}$.

Figure 81 shows some of the measurements of the $4922 \AA\left(4^{1} D \rightarrow 2^{1} P\right)$ excitation function. The polariza- tion of this line is $60 \%$ at threshold and appears to be significant out to several hundred volts. Since the pressures at which Yakhontova, and Zapesochnyi and Feltsan made their measurements are not explicitly stated it is not possible to apply polarization corrections to the different excitation functions. The most complete treatment covering the region of the maximum is the work of Heddle and Lucas, explicitly corrected for instrumental polarization, at an explicitly stated pressure of $1.2 \mu$, coupled with their polarization measurement at $1.3 \mu$ shown in Fig. 73. McFarland (1967) measured the $4^{1} \mathrm{D}$ excitation and polarization using a helium beam. His objective was to study threshold effects. The beam pressure in this work was low enough so that secondary processes would not have been significant. His polarization curve is presented in Fig. 73 . McFarland's $4^{1} D$ excitation function, shown in Fig. 81, and that obtained by Heddle and Lucas are in rather good agreement. Also shown are Moustafa Moussa's results, including cascading, and without polarization correction, for $4^{1} D$ excitation. From 140 to $250 \mathrm{eV}$ Moustafa Moussa, Heddle and Lucas, and McFarland have essentially identical slopes. At lower energies Moustafa Moussa's results are slightly higher than the others.

These results could possibly be reconciled with Yakhontova's results, for example, through depolarization if she worked at higher pressures than Heddle and Lucas, or through some instrumental polarization, which might not have shown up in the ${ }^{1} P$ work where pressure depolarization was more nearly complete. The Zapesochnyi and Feltsan curve is again very different from those of other authors.

Figure 82 shows the $4387-\AA\left(5^{1} D \rightarrow 2^{1} P\right)$ excitation functions of Yakhontova, St. John et al., and Moustafa Moussa uncorrected for cascading or polarization, in comparison with the $5^{1} D$ excitation cross section calculated by Ochkur and Bratsev. The theoretical result characteristically falls below the experimental results at the peak for all the ${ }^{1} D$ lines of helium.

Additional measurements have been carried out for

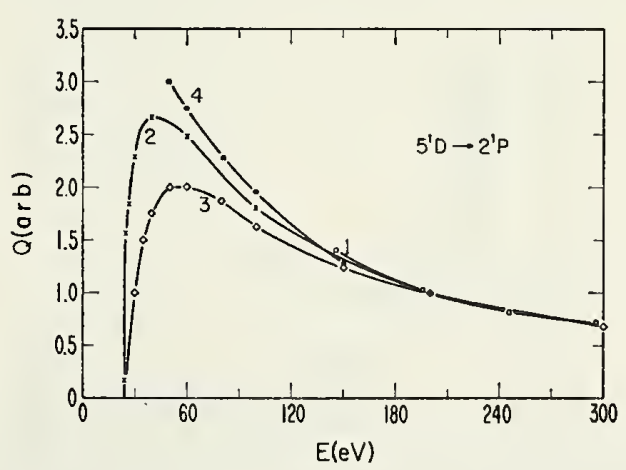

FIG. 82. Relative excitation functions for the helium $5^{1} D \rightarrow 2^{1} P$ transition [St. John, Miller, and Lin (1964), $1.1 \mu$, Curve 1; Yakhontova (1959), Curve 2; and Moustafa Moussa (1967), $1 \mu$, Curve 4] compared with the $5^{1} D$ excitation cross section calculated by Ochkur and Bratsev (1965b), Curve 3. 
$3^{1} D, 6^{1} D, 7^{1} D$, and $8^{1} D$ excitation (St. John et al., Yakhontova, Zapesochnyi and Feltsan, and Moustafa Moussa). St. John and Jobe (1967) have measured a helium excitation cross section at $18695 \AA$. The bandwidth of the monochromator is not given and the paper does not specify whether the measurement includes both singlet and triplet $4 F \rightarrow 3 D$ transitions at 18697 and $18686 \AA$, respectively. They obtained a direct $4 F$ excitation cross section of $17 \times 10^{-20} \mathrm{~cm}^{2}$ at $100 \mathrm{eV}$.

Measured absolute values for ${ }^{1} D$ excitation are compared in Table LXIVc.

\subsection{Helium $n^{3} S \rightarrow 2^{3} P$ Transitions}

The helium ${ }^{3} S$ excitation functions would appear to be as nearly experimentally fool-proof as any excitation function. Since the orbital angular momentum is zero there is no polarization in Russell-Saunders coupling so that all the problems of instrumental polarization, pressure depolarization, and anisotropy do not occur. According to Heddle and Lucas (Fig. 58) the ${ }^{3} S$ and ${ }^{3} P$ excitation functions are not particularly pressure sensitive. The triplet system is free of direct influence of imprisonment of resonance radiation, but is subject to it rather indirectly through collisional transfer of excitation from the singlet system.

The fraction of excitation due to cascading is given as 20 to $30 \%$ for $n^{3} S$ excitation by St. John, Miller, and Lin. This rather large contribution reflects the inaccessibility of the ground state. (The $2^{3} S$ state excitation is a special case and will be discussed separately.) Since ${ }^{3} P$ state excitation function is not pressure sensitive below 7 or $8 \mu$ the component of ${ }^{3} S$ excitation due to cascading should not be pressure-dependent.

The ${ }^{3} S$ excitation functions are displayed in Figs. 83 and 84, and the measured absolute values in Table $\operatorname{LXIV}(d)$.

An intercomparison of the shapes of the excitation functions, and a comparison with theoretical results reveals an interesting difference which seems to be characteristic of the triplet cross sections. The theoretical results are based essentially on an expansion of

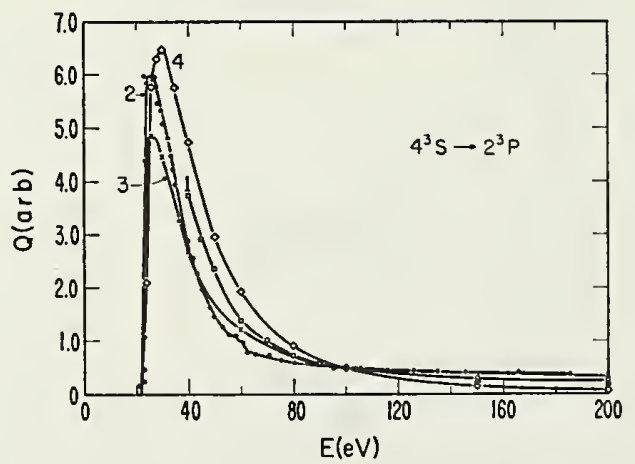

FIG. 83. Relative excitation functions for the helium $4^{3} S \rightarrow 2^{3} P$ transition [St. John, Miller, and Lin (1964), $2.0 \mu$, Curve 1; Heddle and Lucas (1963), $0.5 \mu$, Curve 2; and Yakhontova (1959), Curve 3$]$, compared with the $4^{3} S$ excitation cross section calculated by Ochkur and Bratsev (1965b), Curve 4.

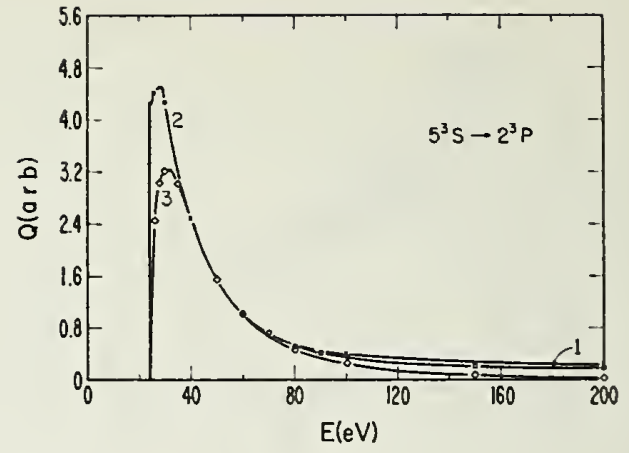

Fig. 84. Relative excitation functions for the helium $5^{3} S \rightarrow 2^{3} P$ transition [St. John, Miller, and Lin (1964), $5.3 \mu$, Curve 1; and Yakhontova (1959), Curve 2] compared with the $5^{3} S$ excitation cross section calculated by Ochkur and Bratsev (1965b), Curve 3. The data obtained by Moustafa Moussa (1967), if normalized t the other curves at $60 \mathrm{eV}$, would correspond closely to Curve 1 at higher energies.

the cross section as a series in the electron energy, $E$. At high energies the leading term, $E^{-3}$, presumably dominates. The experimental results, without exception, fall off much more slowly with energy.

The question arises of whether the slow fall off of the experimental results is instrumental, due to (1) a high background of stray light emitted by the monochromator or (2) excitation by slow electrons produced from the primary beam by scattering at slit edges. Gabriel and Heddle (1960) give a total excitation cross section of $9.6 \times 10^{-18} \mathrm{~cm}^{2}$ for all "observed states," and ${ }^{3} S$ cross sections ranging from 1 to $15 \times 10^{-20} \mathrm{~cm}^{2}$ at $100 \mathrm{eV}$. Therefore a transmission of $1 \%$ in light scattered throughout the spectrum would give the observed high energy tail. This question is not discussed in detail in the papers but there are indications that the background level just off the line in question was checked in most of the work. In this case the theoretician is left with an interesting problem of accounting for a dependence weaker than $E^{-3}$. Perhaps the slow decay may be due to a large coefficient for the term of next higher order in the expansion of the cross sections in powers of $E$. Possibly the slow fall off may arise through cascading from higher levels where a weakening of the RussellSaunders coupling scheme may result in the excitation cross sections having some of the character of singlet excitation.

In Fig. 83 we intercompare some shapes of the $4713-\AA$ $\left(4^{3} S \rightarrow 2^{3} P\right)$ function. The result is disappointing in view of the absence of polarization effects. The pressure at which Yakhontova worked is unspecified. The relatively low peak-to-tail ratio would be consistent with a helium pressure of $10 \mu$ or higher, where according to Fig. 58, the excitation function is enhanced and presumably distorted by excitation transfer. The possibility that space charge effects would account for the difference would not seem likely.

Some further careful work at low pressure, low electron current density, and high electron energy resolution over a broad energy range on the 4713-A excitation 


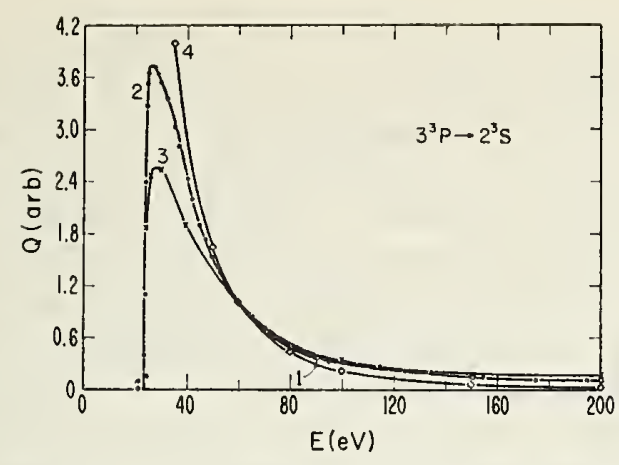

FIG. 85. Relative excitation functions for the helium $3^{3} P \rightarrow 2^{3} S$ transition [St. John, Miller, and Lin (1964), $1.5 \mu$, Curve 1 and $\square$; Heddle and Lucas (1963), $0.25 \mu$, Curve 2; and Yakhontova (1959), Curve 3$]$ compared with the $3^{3} P$ excitation cross section calculated by Ochkur and Bratsev (1965b), Curve 4. Data by Moustafa Moussa (1967), normalized to the other curves at 60 $\mathrm{eV}$, closely correspond to Curve 1 at the higher energies.

function would be very useful. This line provides a good oppurtunity for a highly precise and reliable measurement which would permit a definitive comparison with the theoretical results for triplet excitation, a comparison which may have considerable physical significance.

\subsection{Helium $n^{3} P \rightarrow 2^{3} S$ Transitions}

The ${ }^{3} P$ excitation functions appear to be as insensitive to pressure as are the ${ }^{3} S$ functions. Here, there is the added complication of polarization. This polarization is as high as $25 \%$ at low energies and falls off slowly to zero above $200 \mathrm{eV}$. Therefore, some distortion of the excitation function is possible. The measured excitation functions at $3889 \AA\left(3^{3} P \rightarrow 2^{3} S\right)$ are compared in Fig. 85 and the absolute values of $n^{3} P$ functions in Table LXIVe.

The comparison between the data by Heddle and Lucas and by Yakhontova is much like that of the ${ }^{3} S$ case. The lower peak-to-tail ratio obtained by Yakhontova could be accounted for in terms of polarization only through the assumption that there was nearly $100 \%$ instrumental polarization.

The $2^{3} P \rightarrow 2^{3} S$ excitation measurement at 10 829-30 $\AA$, by Zapesochnyi and Feltsan (1965) is worth special notice. They obtained a value of $2510 \times 10^{-20} \mathrm{~cm}^{2}$ at $26.5 \mathrm{eV}$. Since all $2^{3} P$ excitation decays to the $2^{3} S$ state, the $2^{3} S$ excitation function is necessarily larger than the $2^{3} P$ excitation function. Measurements of $2^{3} P$ excitation functions (Fleming and Higginson, 1964; and Schulz and Fox, 1967) yield values at the peak just above $20 \mathrm{eV}$, a factor of ten smaller. The Zapesochnyi and Feltsan result implies a rapid increase in the $2^{3} P$ cross section and therefore in metastable production in the range from 20 to $26.5 \mathrm{eV}$. The cascade contribution would be quite significant at $26.5 \mathrm{eV}$. Since the measurements were made at pressures of 8 to $25 \times 10^{-3}$ Torr there may also have been significant excitation transfer into the triplet system, much of which would cascade into the $2^{3} P$ state.

\subsection{Helium $n^{3} D \rightarrow 2^{3} P$ Transitions}

The $4^{3} D \rightarrow 2^{3} P$ excitation function at $4471-\AA$ has been shown to be quite pressure sensitive, presumably due to excitation transfer into the higher ${ }^{3} F$ states followed by cascading. This has been discussed in an earlier section. Quite low pressures are required to obtain reliable absolute values, and some slight distortion of the excitation function should be evident at pressures of a few times $10^{-3}$ Torr. Figure 86 displays the excitation function for the $4471-\AA$ line and measured absolute values are listed in Table LXIVf.

Figure 86 illustrates again the remarkable difference between the behavior of the experimental and theoretical results at higher energies. It also shows that the peak-to-tail ratio in Yakhontova's results is still smaller relative to the results of Heddle and Lucas, for this very pressure sensitive line.

\subsection{Excitation of the Helium $n=2$ Levels}

Measurement of helium $n=2$ level excitation presents a special set of problems which have led to a variety of measurement techniques. The lowest excited states are listed below with energies and lifetimes:

\begin{tabular}{ccc}
\hline \hline State & Energy $(\mathrm{eV})$ & Mean life $(\mathrm{sec})$ \\
\hline $3^{3} S$ & 22.72 & $3.6 \times 10^{-8}$ \\
$2^{1} P$ & 21.22 & $5.6 \times 10^{-10}$ \\
$2^{3} P$ & 20.96 & \multicolumn{2}{c}{$1.05 \times 10^{-7}$} \\
$2^{1} S$ & 20.61 & 0.14 \\
$2^{3} S$ & 19.82 & $10^{5}$. \\
\hline
\end{tabular}

As electron energy is increased the metastable states $2^{3} S$ and $2^{1} S$ are excited first, followed by the $2^{3} P$ state

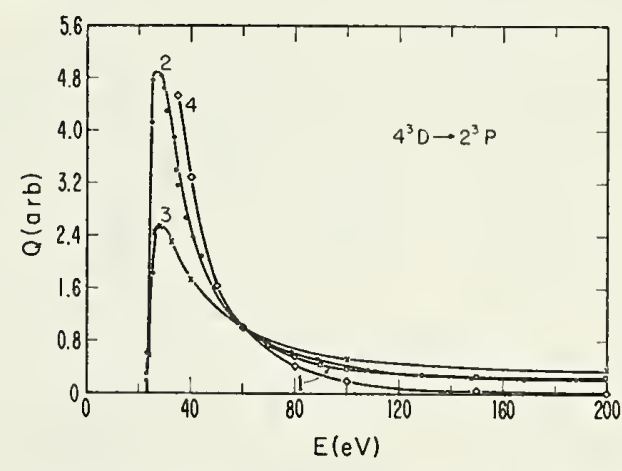

Fic. 86. Relative excitation functions for the helium $4^{3} D \rightarrow 2^{3} P$ transition [St. John, Miller, and Lin (1964), $1.7 \mu$, Curve 1 and $\square$; Heddle and Lucas (1963), $1 \mu$, Curve 2; and Yakhontova (1959), Curve 3] compared with the $4^{3} \mathrm{D}$ excitation cross section calculated by Ochkur and Bratsev (1965b), Curve 4. Data by Moustafa Moussa (1967), normalized to the other curves at $60 \mathrm{eV}$, closely correspond to Curve 1 at the higher energies. 
which decays entirely into the $2^{3} S$ metastable state. At $21.22 \mathrm{eV}$ the $2^{1} P$ state, which radiates primarily to the ground state, is excited. The $2^{1} P \rightarrow 1^{1} S$ transition probability is a thousand times larger than the $2^{1} P \rightarrow 2^{1} S$ transition probability so that the probability of exciting the $2^{1} P \rightarrow 2^{1} S$ line must remain small in the energy interval immediately above its threshold, even for a moderate amount of reabsorption. There is no evidence or expectation of collisional excitation transfer in this energy range, so that observations of excitation over the three volt range from 20 to $23 \mathrm{eV}$ have a simple interpretation in terms of a few processes. Good electron energy resolution becomes of paramount importance in separating the effects of excitation of the different $n=2$ levels.

The oldest method of studying helium excitation in this energy range is due to Maeir-Leibnitz (1935) and was recently used again with refinements of technique by Fleming and Higginson (1964). An axial filament is used in a simple concentric cylindrical configuration of electrodes and grids. Electrons were accelerated to the excitation potential $V$ and emitted through a narrow slit into an excitation volume. An outer collector was biased to receive only elastically scattered electrons. The helium pressure was maintained at 0.47 Torr, at which electrons make a substantial number of collisions before they reach the collector. Near threshold the drop in collector current beyond the first excitation threshold could be analyzed in terms of diffusion theory, taking into account only elastic scattering and excitation of the $2^{3} S$ state. A cross section for excitation of the $2^{3} S$ state at the maximum just above $20 \mathrm{eV}$ of $2.6 \times 10^{-18} \mathrm{~cm}^{2} \pm$ $17 \%$ was obtained.

The other absolute measurement of the $2^{3} S$ peak that is of interest was by Schulz and Fox (1957), using a method of detection of metastables by measurement of the secondary electron current emitted from a metal surface due to energy given up by the metastables. This method was previously exploited by Dorrestein (1942) and subsequently by Holt and Krotkov (1965) for relative measurements.

Figure 87 compares the relative measurements of metastable current from these last three papers. The shapes obtained in the three measurements compare quite well, with differences attributable to differences in energy resolution. Schulz and Fox, using a retarding potential difference method, had an effective energy resolution of about $0.1 \mathrm{eV}$. They used an axially symmetric electrode configuration with the electron beam projected down the axis along a confining magnetic field. The metastable current was measured at an outer cylinder. They put the measurement at the $2^{3} S$ peak on an absolute basis by assuming isotropic motion of the metastables and using an efficiency for electron ejection by metastables at a gold surface obtained by Stebbings (1957). Their result was $4 \times 10^{-18} \mathrm{~cm}^{2} \pm 30 \%$, the stated error overlapping that given by Fleming and Higginson.
The first peak in Fig. 87 is unambiguously attributable to the $2^{3} S$ excitation in the Schulz and Fox experiment because it is well separated from the thresholds for $2^{1} S$ and $2^{3} P$ excitation. Above $20.6 \mathrm{eV}$ the metastable current includes the $2^{1} S$ contribution and the $2^{3} P$ excitation through cascading into the $2^{3} S$. The interpretation of each of the humps and bumps in terms of excitation of a different state has been proved inadequate through the work of Schulz and Philbrick (1964), Chamberlain (1965) and Chamberlain and Heideman (1965), these studies being based on the measurement of components of the scattered electron current corresponding to specific inelastic processes. The Schulz and Philbrick results for electrons scattered at $72^{\circ}$ in an inelastic process leaving the helium atom in the $2^{3} S$ state is included in Fig. 87. These results strongly suggest that the maximum near $21 \mathrm{eV}$ in the total metastable production curve is due to a second peak in the $2^{3} S$ excitation curve rather than being due to $2^{1} S$ or $2^{3} P$ production, and that the bump near $22.5 \mathrm{eV}$ is due to resonance structure. In Fig. 88 results of the five-state close coupling calculation (Burke, Cooper, and Ormonde 1966) discussed in Sec. 3.4 is compared with the experimental results of Schulz and Fox. There is an excellent correspondence between the measured total metastable production cross section and that predicted from the theory by summing the calculated contributions (see Fig. 33), in the range where cascading from higher states does not contribute.

Chamberlain and Heideman obtained a spectacular set of curves for the forward inelastically scattered components corresponding to $2^{3} S, 2^{1} S, 2^{1} P$, and $2^{3} P$ excitation, which demonstrate that these curves are very heavily structured. These are shown in Fig. 89. Both the Schulz and Philbrick, and the Chamberlain and Heideman results were obtained with electron beams of about $0.06 \mathrm{eV}$ half-width. A comparison of their results reveals that the inelastic scattering corresponding to $2^{3} S$ excitation is anisotropic, so the results cannot be applied to obtain a quantitatively correct subtraction of $2^{3} S$ excitation from the total metastable excitation curve.

The work by Holt and Krotkov (1965) is another very significant contribution to the analysis of the metastable production curve. Their total metastable production curve is included in Fig. 87. They capitalized on the fact that the $2^{1} S$ metastables can be quenched with $90 \%$ efficiency in a field of $226 \mathrm{kV} / \mathrm{cm}$. The quenched component of the production provided a cross section for the $2^{1} S$ state excitation, which rises smoothly from threshold to a broad maximum centered at $22 \mathrm{eV}$ and with about one-third the amplitude of the first $2^{3} \mathrm{~S}$ maximum. The unquenched component gave a total cross section for excitation of the $2^{3} S$ and $2^{3} P$ states, since all the $2^{3} P$ atoms immediately decay into the $2^{3} S$ state, and no higher triplet states are excited in the energy range up to $22.7 \mathrm{eV}$.

Holt and Krotkov also used an inhomogeneous magnetic field to spatially separate the three components of 


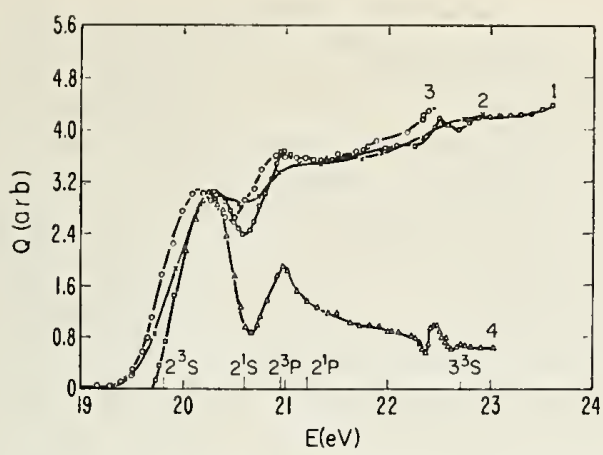

FIG. 87. The total helium metastable excitation functions measured by Schulz and Fox (1957), Curve 1 [the data were taken from Schulz and Philbrick (1964)]; Holt and Krotkov (1966), Curve 2; and Dorrestein (1942), Curve 3 are intercompared on a relative basis. Curve 4 is the $2^{3} S$ excitation cross section measured at $72^{\circ}$ by Schulz and Philbrick (1964).

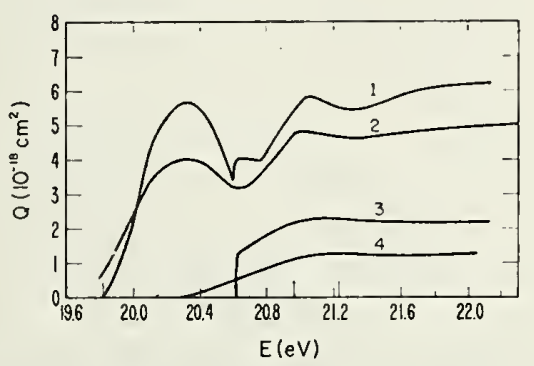

Fig. 88. The total helium metastable production cross section obtained by Schulz and Fox (1957), Curve 2, is compared with the combined results of five-state close coupling calculations (Burke, Cooper, and Ormonde, 1966), Curve 1 . The $2^{1} S$ production cross section obtained by Holt and Krotkov (1966), Curve 4, is also included, in comparison with the $2^{1} S$ excitation cross section calculated by Burke, Cooper, and Ormonde, Curve 3.

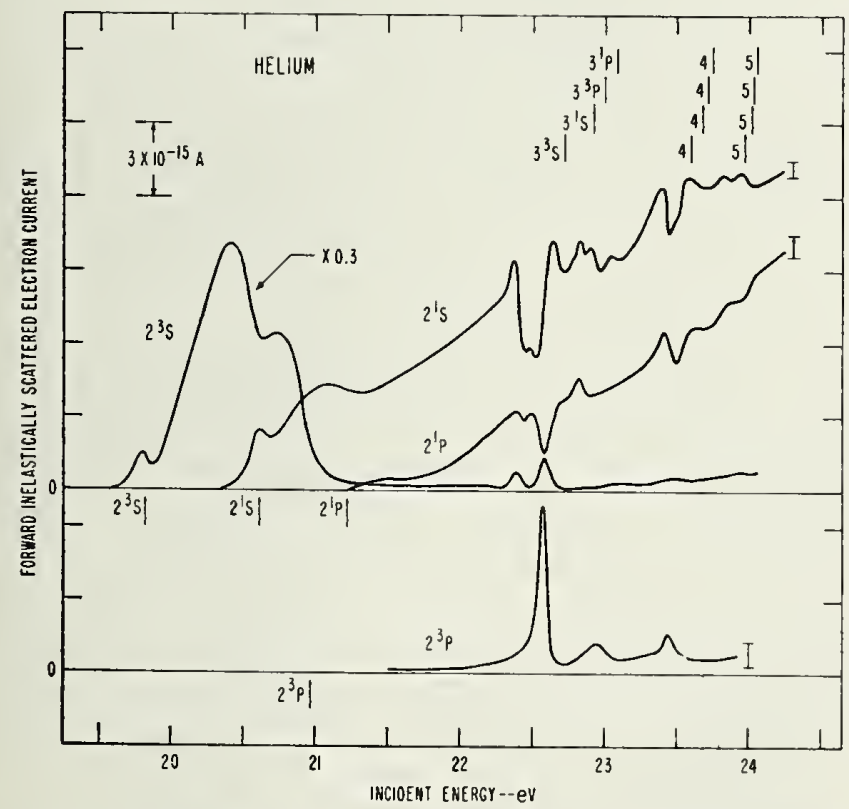

Fig. 89. Energy dependences of the components of forward inelastically scattered electron current in helium corresponding to excitation to the $2^{3} S, 2^{1} S, 2^{3} P$, and $2^{1} P$ states. The curves are smoothed tracings of the original data, and the width of the noise is indicated by the error bars. (Chamberlain and Heideman, 1965). the ${ }^{3} S$ state. The metastables were allowed to pass through an aperture out of the excitation region into a drift space at lower background pressure, where the magnetic and electric fields could be applied. At the end of the drift space the metastables were detected by counting the electrons ejected from a tungsten electrode placed in the path of the beam.

From the $2^{3} S$ differential excitation cross sections shown in Figs. 87 and 89 they inferred a total $2^{3} P$ excitation cross section which they used as the basis for calculating the polarization of those $2^{3} S$ atoms derived by cascading from the excitation of the $2^{3} P$ state.

Cêrmak (1966) developed a method of analyzing metastable production by energy analyzing the electrons, with a Lozier technique, produced by Penning ionization as a metastable beam is passed through a gas of lower ionization potential. For his study of helium excitations he used argon, with an ionization potential of $15.75 \mathrm{eV}$. The $2^{3} S$ states $(19.82 \mathrm{eV})$ result in electrons of $4.07 \mathrm{eV}$ and the $2^{1} S$ states $(20.61 \mathrm{eV})$ result in $4.86-\mathrm{eV}$ electrons. Retarding potential analysis was used to determine the electron currents in each energy component. The behavior of these electron currents as a function of the energy of the exciting electron beam yields excitation cross sections for the two states. The results are relative cross sections but they could be related to each other if the cross sections for Penning ionization were known.

Cêrmak extended his results up to $60 \mathrm{eV}$ so that his ${ }^{3} S$ excitation function includes the cascading from all excitation into the triplet system. Similarly, the ${ }^{1} S$ excitation function necessarily includes a significant cascading contribution from higher ${ }^{1} P$ states. The pressure in the source, $5 \times 10^{-4}$ to $10^{-3}$ Torr, was high enough so that some modification of the singlet system cascading pattern presumably occurred due to reabsorption of resonance radiation.

Dugan, Richards, and Muschlitz (1967), using a system somewhat similar to that of Holt and Krotkov, studied the excitation functions of the $2^{1} S$ and $2^{3} S$ states at electron energies of 25 to $135 \mathrm{eV}$. They used an inhomogeneous magnetic field to deflect the $M_{s}= \pm 1$ components of the $2^{3} S$ state out of the beam. It was assumed that this reduces the $2^{3} S$ population by twothirds, leaving the one-third in the $M_{s}=0$ substate undeflected. By this method they could determine the separate excitation functions and their ratios. However, the analysis depended on extrapolation to zero pressure from excitation over a helium pressure range of 40 to $130 \mu \mathrm{Hg}$, in order to eliminate effects of imprisonment and differential scattering. For their geometry it seems certain that imprisonment is severe over the whole range of pressures used, and an extrapolation to zero pressure cannot be reliable. As Phelps (1958) has demonstrated, the cross section for excitation of the $5016 \AA\left(3^{1} P \rightarrow 2^{1} S\right)$ line is tremendously enhanced at helium pressures of $40 \mu$, to the point that it alone is 
comparable to the value of the $2^{1} S$ cross section estimated by Holt and Krotkov. The imprisonment process is not linear at high pressures. An opposing error should also occur due to enhancement of the $2^{3} S$ state as a result of excitation transfer into the triplet system. The observations by Lin and St. John (see Fig. 56) show that this would result in large contributions to the triplet population at high pressure and at the higher electron energies. This process would be quadratic in pressure, not linear.

\subsection{Thresholds of Helium Excitation Functions}

The discussion of the $n=2$ excitation functions has centered on behavior near threshold, and has shown that this is a region of special interest. This interest also exists in thresholds for the excitation of higher levels of helium. In the work which has been discussed on the excitation of the visible helium lines thresholds have not been emphasized but rather we have been concerned with the determination of the gross behavior of the excitation functions. There has been a considerable amount of threshold work, especially as it has related to the polarization of the excited radiation near threshold.

Perhaps the first experimental work to reveal the general behavior of the thresholds was by Yakhontova (1959). Detailed shapes were obtained by Smit, Heideman, and Smit (1963). Most of these results were obtained at pressures of 2 to $10 \mu \mathrm{Hg}$. Zapesochnyi and Feltsan (1965) measured the shapes of a large number of thresholds at pressures from 0.3 to $3 \mu$. None of these workers considered effects due to the polarization of the radiation. All used the thermal energy width of electrons from a hot cathode with no further energy selection. Energy resolution was about a third of an electron volt for the two more recent works while Yakhontova, using a more intense electron beam, had resolutions ranging from 0.7 to $1.2 \mathrm{eV}$.

Zapesochnyi and Shpenik (1966), using a $127^{\circ}$ electrostatic analyzer, studied threshold excitation for a number of atoms. They give results for the threshold behavior of $3^{3} S, 4^{3} S, 3^{3} P, 3^{3} D$, and $3^{1} D$ excitation obtained with electron beam half-widths of 0.3 to $0.5 \mathrm{eV}$, showing pronounced structure immediately at threshold.

Heddle and Keesing (1967a) have applied a hemispherical electron monochromator to the study of the thresholds for $4^{1} S$ and $4^{3} S$ states. The energy spread of electrons transmitted by the monochromator was nominally $0.070 \mathrm{eV}$. The $4^{1} S$ and $4^{3} S$ functions had much the same behavior, each exhibiting a second maximum beyond the threshold maximum, which Heddle and Keesing suggest is a resonance with the configuration $\left(1 s 5 s^{2}\right)^{2} S_{1 / 2}$.

To illustrate the threshold behavior Fig. 90 displays the results obtained by Smit, Heideman, and Smit for one line from each series. General characteristics of thresholds within a spectral series seem to be the same.

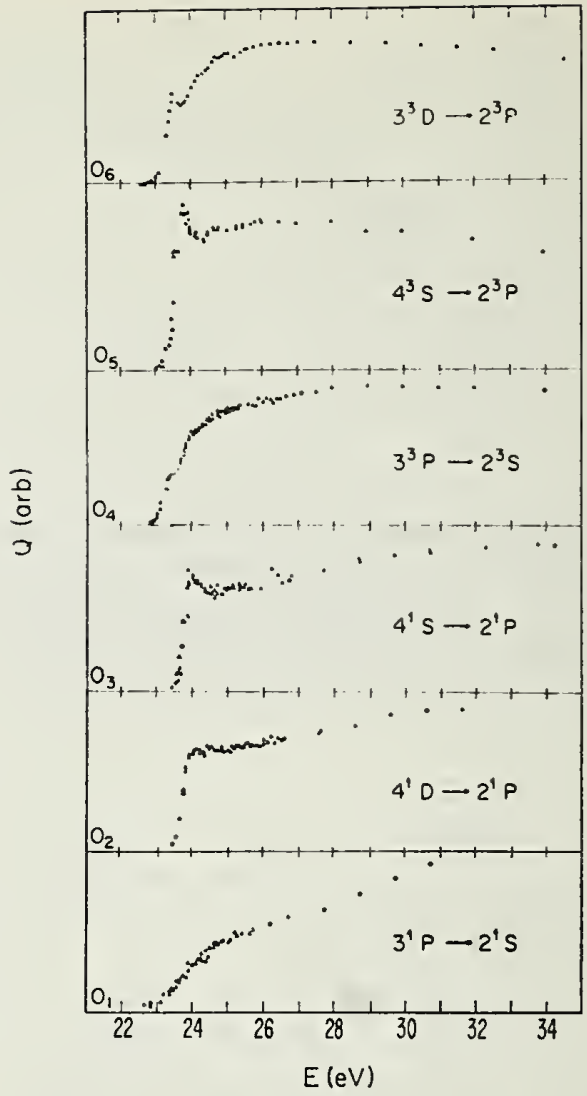

Fig. 90. Threshold behavior of the helium singlet and triplet series excitation functions obtained by Smit, Heideman, and Smit (1963).

An exception occurs for the ${ }^{3} D$ series, the structure in the $3^{3} D \rightarrow 2^{3} P$ excitation function being absent from the $4^{3} D$ and $5^{3} D$ functions.

Polarization of helium lines excited by electron impact has been a controversial subject since Lamb and Maiman (1957) published a measurement of the polarization of the helium $3889-\AA\left(3^{3} P \rightarrow 2^{3} S\right)$ line, showing a minimum at $28 \mathrm{eV}$. Earlier work by Skinner and Appleyard (1927) showed that the polarization of electron impact excited radiation for a number of mercury lines drops toward zero as the electron energy approaches threshold from above.

In 1958 Percival and Seaton published the theoretical study of polarization of impact radiation discussed in Sec. 9. In their paper Percival and Seaton reemphasized the fact that for many lines conservation of angular momentum dictates the value of polarization these lines must have when excited by an electron carrying only the energy of excitation and no more. Further, they pointed out that the results of Skinner and Appleyard seemed to violate these essential requirements by going toward zero at threshold, and they suggested that more careful experimental work seemed in order.

Heddle and Lucas (1961; 1962) and McFarland and Soltysik $(1961 ; 1962)$ carried out their studies of polarization using helium, and both studies showed polarization in helium going to zero at threshold. The results have been reproduced in Fig. 73. 
Hughes, Kay, and Weaver (1963) obtained similar behaviors at threshold. Their work and the results of $\mathrm{McF}$ arland and Soltysik showed in excellent detail the structure of the $3^{3} P \rightarrow 2^{3} S$ polarization near threshold first observed by Lamb and Maiman. However, the work of Hughes, Kay, and Weaver suffers from instrumental effects similar to but more severe than those appearing in McFarland and Soltysik's results. In both, there are cases of negative polarization increasing with pressure at fixed electron energy, which must be a nonphysical effect.

The polarization observed $\left(P_{\text {obs }}\right)$ at $90^{\circ}$ to the electron beam, through a monochromator, is related to the true percentage polarization $P$ of the line in equation (169). We rewrite Eq. (169) to include depolarization effects.

$$
\frac{P_{\text {obs }}}{100}=\frac{(\gamma P / 100)+\left[\left(T_{11}-T_{\perp}\right) /\left(T_{11}+T_{\perp}\right)\right]}{1+(\gamma P / 100) \cdot\left[\left(T_{11}-T_{\perp}\right) /\left(T_{11}+T_{\perp}\right)\right]} .
$$

Here, $\gamma$ is a pressure depolarization factor which may take values between zero and unity. At high pressures depolarizing collisions and depolarization due to such processes as imprisonment of resonance radiation and collisional transfer of excitation, may occur. The factor $\gamma$ becomes small as such effects become dominant and the observed polarization at sufficiently high pressure is the instrumental polarization. Instrumental polarization is also observed at energies such that the true polarization is zero.

The observations of small values of polarization near threshold have been the subject of continuing controversy. A simple integration sufficies to show that the observed effects cannot be due merely to the finite energy distribution in the electron beam. McFarland (1963, 1964) carried out an extensive series of tests to determine whether the observations might be the result of radially scattered electrons. In the course of this work he found low polarization values near threshold for $3^{3} P, 3^{1} P, 5^{1} D$, and $4^{1} D$ excitation, but the polarization seemed to climb toward the theoretical value again, very near to the threshold.

Heddle and Keesing (1964, 1965) have confirmed this behavior for the $3^{1} P$ and $4^{1} D$ excitations, and Soltysik, Fournier, and Gray (1966) have reproduced $\mathrm{McF}$ arland's results for $3^{1} P, 3^{3} P$, and $4^{1} D$ excitation. More recently McFarland (1967) has carried out a careful crossed beam measurement of the polarization of the $4922-\AA\left(4^{1} D_{2} \rightarrow 2^{1} P_{1}\right)$ line of helium. The results near threshold, shown in Fig. 91, confirm the earlier measurements. Heddle and Keesing (1967b) have also carried out very careful studies of $4^{1} D, 3^{1} P, 3^{3} P$, and $4^{3} S$ threshold polarization. The predicted zero polarization of the $4^{3} S$ case was confirmed. For the states with nonzero angular momentum, minima as much as several volts wide near threshold appear to be established as physical properties of the excitation process, and the indications are that the threshold polarizations required by conservation of angular momentum are satisfied by rapid increases in polarization within the last few tenths of a volt above threshold.

\section{Heavy Rare Gases}

The first significant contribution on the excitation functions of neon and argon came out of the work of Hanle (1930), at Jena, who studied the excitation functions of neon at rather high pressures. He described some strong pressure dependences. Subsequently Fischer (1933), also at Jena, studied the excitation functions for a number of lines of neutral argon originating in the $n=5$ shell and terminating in the $n=3$ shell. He also studied a number of lines of ionized argon excited from the neutral ground state. Herrmann (1936) obtained excitation functions for a large number of argon and neon lines. The pressures used were approximately $20 \mu$ for argon and somewhat higher for neon.

Maeir-Leibnitz (1935) obtained threshold measurements on neon and argon similar to those already described for helium, and Dorrestein (1942) studied metastable production in neon. Milatz and Ornstein (1935) observed absorption of the 6402- $\AA$ line to determine the neon metastable concentration in an electronic excitation measurement. Milatz and Woudenberg (1940) obtained absolute values for four neon excitation functions, referring the measurements to a standard tungsten lamp.

It would be impossible to critically evaluate these data. The pressures used were in the range for which excitation transfer and imprisonment of resonance radiation are important for helium. There have been no detailed quantitative studies of pressure-dependent effects, analogous to those which have been carried out for helium. The data were taken without regard to the state of polarization of the observed radiation. Furthermore, there has been surprisingly little duplication of

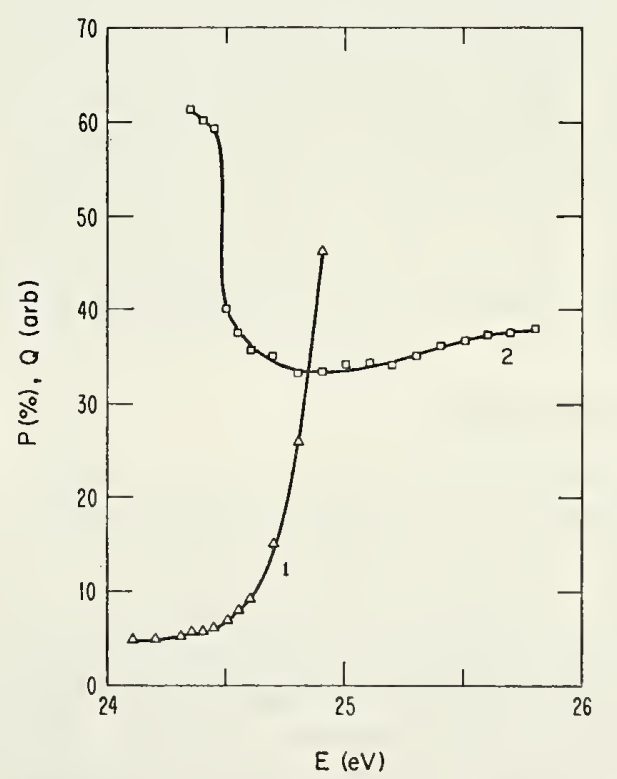

FIG. 91. Threshold polarization, curve 2, and excitation, curve 1, of the helium $4^{1} D_{2} \rightarrow 2^{1} P_{1}$ line obtained in a crossed beam measurement (McFarland, 1967). 
measurements so that intercomparison of data is not productive.

Data taken in the last decade are on a better basis because lower pressures were used to reduce secondary effects. However, there is still little duplication, and there has been no recognition of the problems of polarization and imprisonment in this work on the heavy rare gases.

Devyatov and Kaptsov (1955) carried out relative measurements of krypton excitation functions at about $10^{-3}$ Torr. Excitation functions were obtained for ten neutral krypton lines and four lines of singly ionized krypton. Photographic detection was used. In 1957 Sin San Guk and A. M. Devyatov published further relative measurements of krypton excitation functions, also including some work on xenon. Pressures were again about $10^{-3}$ Torr. These measurements were carried out in sealed tubes, using a barium getter. Photoelectric detection was used.

Volkova (1959) measured shapes of six argon excitation functions at pressures of $1.0 \times 10^{-3}$ Torr, only one of which is presented in her paper. Volkova and Devyatov (1959) measured the absolute values of these six lines and one other line at argon pressures of $4.68 \times 10^{-3}$ Torr. They calibrated against a tungsten lamp, and commented that their results for the $7723-4-\AA$ argon line agreed with the absolute value obtained by Herrmann in 1936. Volkova, Devyatov, and Kuralova (1960) measured absolute values of several lines of krypton and xenon. Zapesochnyi and Feltsan (1963), measured relative excitation functions for three neon lines.

A number of these recent workers have commented on the characteristic differences between helium excitation and the excitation functions of the heavier rare gases. Russell-Saunders coupling holds for only a few levels for these heavier gases, and intercombination lines are common. Triplet excitation does not have the strongly resonant character seen in helium. Zapesochnyi and Feltsan (1966) presented shapes and absolute values for excitation cross sections of the ten Paschen levels $\left(2 p_{n}\right)$ of argon, and showed an interesting comparison of the behavior of the $2 p_{8}$ levels of argon, krypton, and xenon, which we show in Fig. 92. These excitation functions peak more strongly toward threshold at higher atomic masses.

\section{Alkali Atoms}

The properties of the light alkali metals are of much interest from several points of view. They are, to a reasonable approximation, one-electron systems and have been investigated theoretically by a number of workers (see Sec. 6). They are particularly interesting as components of stellar atmospheres and other plasmas, in part because their low ionization potentials $(\sim 4-5$ $\mathrm{eV}$ ) lead to high ionization probabilities and put them in the role of electron donors. Also, in contrast to hydrogen, the resonance lines occur in the visible or quartz

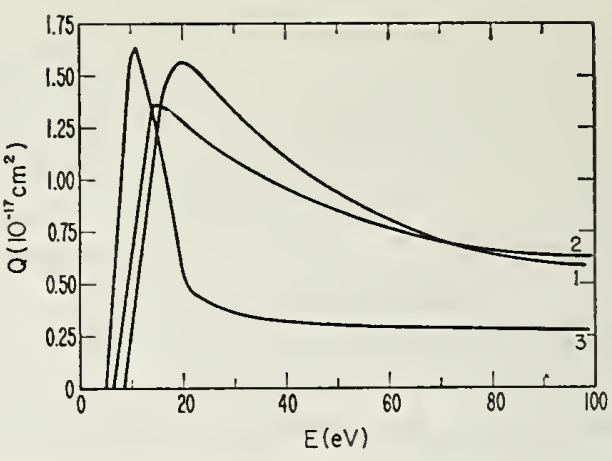

FIG. 92. Measured absolute excitation functions of the (Paschen) $2 p_{8}$ levels of argon $(z=18)$, Curve 1 ; krypton $(z=36)$, Curve 2; and xenon $(z=54)$, Curve 3 . (Zapesochnyi and Feltsan, 1966).

ultraviolet and may be prominent features of visible stellar spectra.

Experimentally the alkalis present a unique set of problems. Appreciable vapor pressures of these metals can be achieved at temperatures of a few hundred degrees centigrade, and most of the excitation work has been carried out in apparatus quite similar to that described for helium except that it is operated at elevated temperatures. Typically, the reservoir of alkali metal is placed in a sidearm the temperature of which is controlled to achieve a desired vapor pressure. The rest of the apparatus is usually operated at a somewhat higher temperature to avoid condensation on the walls. At the temperatures often used the experimental difficulties include the fact that glass becomes electrically conducting so that electrical measurements become difficult. Alkali vapors are readily absorbed in glass causing optical windows to deteriorate. This absorption also causes possible difficulties in attaining equilibrium.

Some attention has been given to the possibility of dimer content in alkali atmospheres. Calculations based on thermal equilibrium indicate that the percentage of atoms in the molecular form is typically a fraction of one percent at operating temperatures and increases with temperature and vapor pressure. Serious contamination of atomic excitation functions would require that the molecular excitation cross sections be large, and would depend on the spectral resolution of the detection equipment. Generally, it is probably not a serious problem where high resolution spectrometers are used but must be considered in each case. The effects of reabsorption of resonance radiation must also be considered. Another complication is in the difficulty of operating electron guns at energies down to one or two volts in order to observe the excitation threshold. Some of these experimental problems are circumvented for measurements carried out with beams of alkali atoms, but only a few such studies have been made.

For lithium the only available results were obtained using lithium beams, because of the high temperature $\left(400^{\circ}-500^{\circ} \mathrm{C}\right)$ required to obtain the necessary static pressure. The crossed beam results are all relative, no absolute beam measurements having been attempted. 


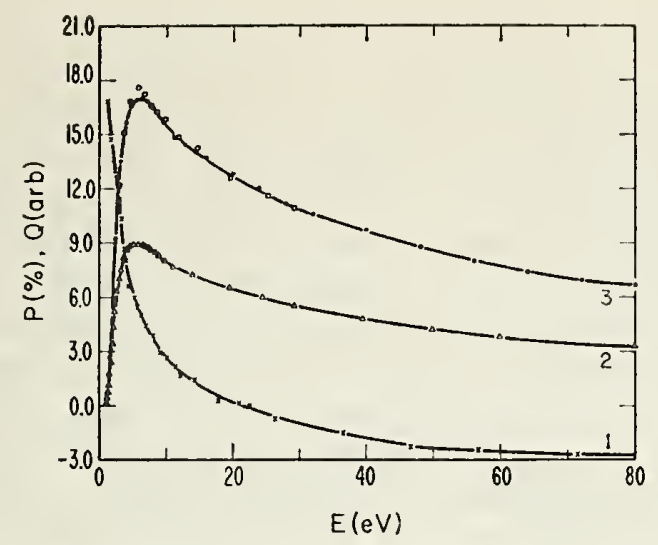

FIG. 93. Polarization (Curve 1) and relative intensity component $I_{\|}$(Curve 2$)$ of the first resonance line $(2 p \rightarrow 2 s, 6708-\AA$ ) of lithium (natural isotopic composition) excited by electron impact, measured by Hafner and Kleinpoppen (1967). Curve 3, the total intensity observed at $90^{\circ}$ to the electron beam, is calculated from Curves 1 and 2. Also shown on Curve 3 are the data of Hughes and Hendrickson (1964), $\square$.

Hughes and Hendrickson (1964) used a lithium beam crossed by an electron beam to measure the relative excitation cross section for the $2 p \rightarrow 2 s$ line at $6708 \AA$ (the $2 p \rightarrow 1 s$ line in terms of a hydrogenic scheme). An interference filter was used so that the doublet was not resolved. Beam density and the possibility of reabsorption of resonance radiation were not discussed.

In the course of studying the polarization of radiation excited by electron impact, Hafner and Kleinpoppen (1967) have obtained an excitation curve for the 6708 - $\AA$ line over the range from threshold to about $80 \mathrm{eV}$, using an interference filter to isolate the line. Their published results show the intensity of the polarization component with electric vector parallel to the electron beam. The measured function is easily corrected using their measured polarization to obtain the total intensity. This measurement and that of Hughes and Hendrickson were carried out on lithium beams composed of natural abundances of the isotopes. The results of both measurements are shown in Fig. 93. The agreement is quite good. In connection with their measurement Hafner and Kleinpoppen showed that depolarization of the radiation occurred as the beam density was increased, due to reabsorption and reemission of the resonance radiation within the beam. Their results were obtained at pressures low enough so that the effect was negligible. The effect of such trapping on the observed excitation curve for a resonance line would be threefold: it would effectively increase the volume from which the radiation was observed (although in a beam experiment convenient limits are provided by the size of the beam), it would depolarize the radiation, and it would result in an increase in the cascading contribution due to an effective modification of branching ratios from the higher $p$ states.

Hafner, Kleinpoppen, and Kruger (1965) measured an excitation function for the $3^{2} D \rightarrow 2^{2} P$ (6103- $\AA$ ) line of lithium and observed no depolarization as a function of beam pressure, consistent with the fact that the $3^{2} D$ state does not optically connect to the ground state and is affected only very indirectly through cascading with imprisonment effects. Their results are shown in Fig. 94.

The most important result presented in these papers by Kleinpoppen and his students is a measurement of the threshold polarization of $\mathrm{Li}^{6}, \mathrm{Li}^{7}$, and $\mathrm{Na}^{23}$. Their results for the resonance lines show that the polarizations decrease monotonically from threshold as a function of electron energy, and that the three threshold polarizations obtained agree with the three values predicted from the theory of Percival and Seaton, these being different primarily because of differences in widths and separations of fine and hyperfine levels in the different isotopes. However, the polarization of the non-resonant $3^{2} D \rightarrow 2^{2} P(6103-\AA)$ line appears to fall below the theoretical value at threshold.

Feldman and Novick (1963) have carried out experiments which demonstrate the existence of long-lived excited states in lithium, potassium, and rubidium. These states are presumed to be associated with the excitation of core electrons, rather than the valence electron. The energies of such states are above the ionization energy associated with the valence electron but below the second ionization potential, and therefore may be autoionizing states. By collecting either the ions or electrons produced in the autoionization process, Feldman and Novick obtained excitation functions and, by changing the distance from the excitation region to the detector, obtained measurements of the effective lifetimes.

All measurements of excitation functions of sodium and the heavier alkalis were carried out using static systems rather than beams.

As in the case of lithium the reabsorption of resonance radiation in sodium should be significant down to quite low pressures. The effect of this on the first reso-

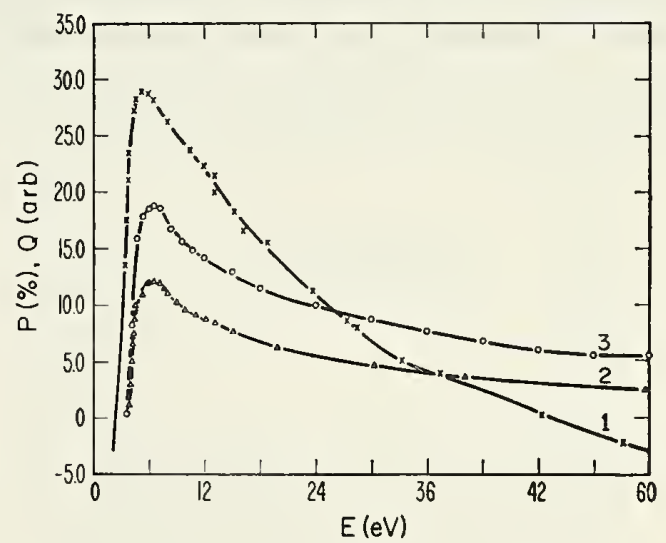

FIG. 94. Polarization (Curve 1) and relative intensity component $I_{||}$(Curve 2) of the $6103-\AA\left(3^{2} D \rightarrow 2^{2} P\right)$ line of lithium (natural isotopic composition) excited by electron impact, measured by Hafner, Kleinpoppen, and Kruger (1965). Curve 3, the total intensity observed at $90^{\circ}$ to the electron beam, is calculated from Curves 1 and 2. 
nance line would be to increase the source volume, to produce some modification of the cascading patterns, and to depolarize the radiation.

Hafner, Kleinpoppen, and Kruger (1965) have measured the polarization of the sodium $D$ lines and found that it decreases monotonically with energy from about $15 \%$ at threshold. This results in an anisotropic radiation pattern as indicated by equation (158) but the polarization is not large and the difference $I\left(90^{\circ}\right)-\bar{I}$ is $5 \%$ or less of $\bar{I}$. The distortion due to instrumental polarization could be $15 \%$ at most, and this only in the case of nearly complete instrumental polarization.

The other sodium lines will be subject to greater errors. Measurements of absolute values are subject to modification of the effective branching ratios when imprisonment of the higher resonance lines occurs. The effects of excitation transfer would be apparent in distortion of nonresonant lines, but most experimental work has been carried out at pressures low enough so that this should not be important.

In general, the shapes of alkali excitation functions determined experimentally should be somewhat less subject to imprisonment and polarization effects than helium, while the measured absolute values should be quite sensitive to the effects of imprisonment of resonance radiation. Unfortunately, there has not been enough experimental work directed at evaluation of these pressure and polarization effects. There has not been enough work for intercomparison to indicate reproducibility. Much of the available work is confined to a narrow energy range near threshold, where theoretical work is likely to be unreliable, so that comparisons with theoretical shapes over the narrow energy range available are not very illuminating.

Zapesochnyi and Shimon (1962) studied the dependence of the shape of the excitation function for the first sodium resonance line on pressure. The results are shown in Fig. 95. The measurements at $2.5 \times 10^{-4}$ and $5 \times 10^{-4}$ Torr are in agreement, while the curve obtained at $10^{-3}$ Torr falls more rapidly with energy. This

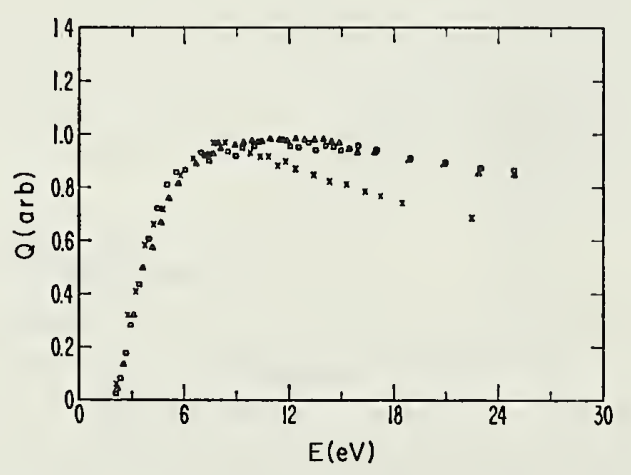

Frg. 95. Behavior of the excitation function of the resonance line of atomic sodium as a function of pressure: $2.5 \times 10^{-4}$ Torr $(\square), 5 \times 10^{-4}$ Torr $(\triangle)$, and $1 \times 10^{-3}$ Torr $(X)$. The electron current density was $1.5 \times 10^{-4} \mathrm{~A} / \mathrm{cm}^{2}$. (Zapesochnyi and Shimon, 1962). behavior is difficult to explain. Modification of cascading patterns by imprisonment of resonance radiation would seem not to provide an explanation. The possibility of contamination of the spectrum, as from the sodium dimer, does not seem likely. Under the experimental conditions the dimer content is a few tenths of one percent and rises with temperature as is easily demonstrated from simple thermodynamic calculations. A dimer excitation cross section peaked strongly at threshold would conceivably give the observed result. A more reasonable possibility is that the trapping of resonance radiation so extends the effective life of the upper state at $10^{-3}$ Torr that chemical interactions between excited states of sodium provide an energy-dependent mechanism for depleting the population of the upper state of the $D$ line. In any case it is reasonable to accept the shape obtained at 2.5 and $5 \times 10^{-4}$ Torr pending a more adequate understanding of the processes which cause the distortion.

Haft (1933) measured the excitation functions of a number of sodium lines, but worked at rather high pressures. Christoph (1935) measured the absolute.cross section for excitation of the sodium $D$ lines using a tungsten strip lamp for radiometric calibrations, and working at a side-arm temperature of $163.5^{\circ} \mathrm{C}$. Volkova (196i) measured absolute values of ten sodium lines at temperatures of $182^{\circ}$ and $202^{\circ} \mathrm{C}\left(p \simeq 5 \times 10^{-5} \mathrm{~mm} \mathrm{Hg}\right)$. Because of window darkening due to sodium absorption the intensities were determined by comparison with lines excited in helium at a pressure of $4 \times 10^{-3}$ Torr. Absolute measurements of helium excitation by Yakhontova (1959) were used to provide the calibration. This indirect calibration process led to stated errors of about $70 \%$.

The most extensive set of measurements under conditions of reasonably low pressure have been carried out by Zapesochnyi and Shimon (1965). They give errors of $30-35 \%$ in absolute values and relative errors not exceeding $10 \%$.

The absolute value of the sodium $D$ line excitation function was measured by Zapesochnyi and Shimon (1965) at $164^{\circ} \mathrm{C}$, essentially the same temperature as was used by Christoph. In fact, Zapesochnyi and Shimon used an absorption coefficient of $0.4 \mathrm{~cm}^{-1}$ determined by Christoph to correct for absorption of resonance radiation, so the two measurements, carried out at the same pressure, are not completely independent. Figure 96 shows the absolute excitation functions obtained by Zapesochnyi and Shimon for the principle series of sodium. Christoph's three absolute measurements for the $D$ line, are shown there for comparison. We have also included Born approximation results (Vainshtein, 1965) and the results of a close coupling calculation by Barnes, Lane, and Lin (1965).

Christoph gave his results in terms of $\mathrm{cm}^{2} / \mathrm{cm}^{3}$ per electron at one millimeter pressure. He gave his working pressure as $1.6 \times 10^{-5}$ Torr at $163.5^{\circ} \mathrm{C}$, which is reason- 


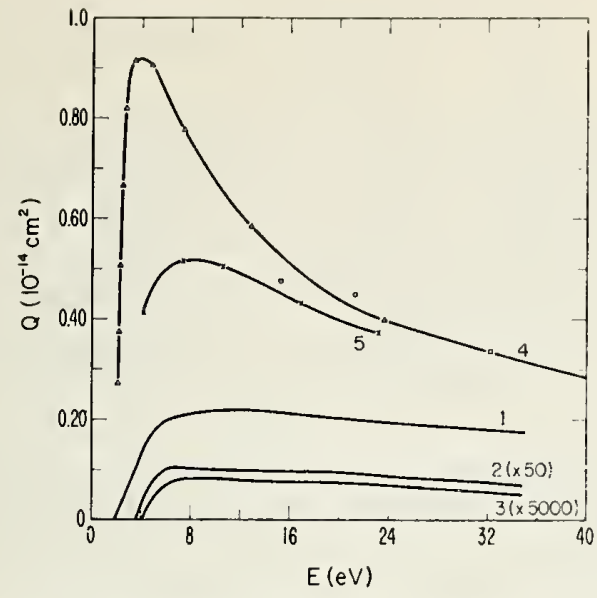

Fig. 96. Absolute excitation function of the resonance line of atomic sodium $(5890-96 \AA, 3 p \rightarrow 3 s)$. Included are three points by Christoph (1935) ( $\square$ ); the excitation function measured by Zapesochnyi and Shimon (Curve 1); the Born approximation cross section for direct excitation of the $3 p$ state, not including cascading, by Vainshtein (1965) (Curve 4); and a close coupling result, not including cascading, by Barnes, Lame, and Lin (1965) (Curve 5). Also shown are excitation functions for the sodium $4 p \rightarrow 4 s$ and $5 p \rightarrow 5 s$ transitions (Curves 2 and 3 , respectively) measured by Zapesochnyi and Shimon (1965).

ably consistent with more recent vapor pressure tables. From a summary of vapor pressure data by Hultgren, Orr, Anderson, and Kelly (1963) we obtain a vapor pressure of $1.7 \times 10^{-5}$ Torr, and the difference is not significant. Christoph's statistical accuracy was of the order of $25 \%$ to which must be added basic radiometric errors and systematic pressure errors. He gave values of 105 (at $11 \mathrm{eV}), 99($ at $21 \mathrm{eV}$ ), and $74($ at $32 \mathrm{eV}$ ) in units of $\mathrm{cm}^{2} / \mathrm{cm}^{3}$. According to Kieffer and Dunn, gas density for an ideal can be computed from

$$
\rho=\left(3.535 \times 10^{16}\right) P_{c}\left(273 / T_{c}\right) \text { atoms }-\mathrm{cm}^{-3},
$$

where $P_{c}$ and $T_{c}$ are the pressure in Torr and temperature in ${ }^{\circ} \mathrm{K}$, respectively. Use of this expression permits conversion of the data to $\mathrm{cm}^{2}$ per atom yielding 48,45 , and $34 \times 10^{-16} \mathrm{~cm}^{2}$. Bates, Fundaminsky, Leech, and Massey (1950) published Christoph's results plotted in a figure at values of $46.6,44.2$, and $32.6 \times 10^{-16} \mathrm{~cm}^{2}$, which have been widely quoted. Zapesochnyi and Shimon (1965) have erroneously remarked that values in use in the literature, were a factor of almost two too high, but they have evidently overlooked the factor $273 / T_{c}$ in the density formula.

This being the case, it is possible that Zapesochnyi and his colleagues have not correctly interpreted their own experimental work on sodium and, perhaps, the other alkali atoms. For excitation of the sodium $D$ line their absolute values would be too low by the factor $273 / 437=0.625$. As can be seen from Fig. 96, such an adjustment would bring their excitation function into better agreement with the theoretical results at higher energies. The data for excitation of alkali atoms presented in this review, however, are taken without modi- fication from the papers by Zapesochnyi and his colleagues.

Figures 97 and 98 give the absolute results obtained by Zapesochnyi and Shimon for the sharp and diffuse series of sodium. These results were obtained with pressures not greater than $4.5 \times 10^{-4}$ Torr and with energy spreads of not more than $0.8 \mathrm{eV}$. In Table $\mathrm{LXV}$, taken from Zapesochnyi and Shimon (1965), their absolute values for the sodium photon-excitation cross sections, taken at cross section maxima, are compared with results of Christoph (1935) and of Volkova (1961) (a corrected value is substituted for the last figure in the column, an error having occurred in the English translation).

Zapesochnyi and Shimon (1965) investigated the dependence of the absolute values, within a given spectral series, on the principal quantum number $n$ of the upper level. They found a good correspondence to the relation $Q(n)=c / n^{\alpha}$, where for the diffuse series $\alpha=5.25$ and $c=5.56 \times 10^{-4}$; and for the sharp series $\alpha=6.87$ and $C=1.26 \times 10^{-12}$. A departure is noted for the excitation of the lowest $D$ level, presumably because this lies in the path of cascade transitions from higher $F$ levels.

The rather high values they obtain for $\alpha$ imply that cascading contributions can be estimated on the basis of only a few higher levels. For example, from Table LXV and Figs. 96 and 98 it can be seen that the resonance line contains a contribution of about $25 \%$ due to cascading from the $D$ states. Other cascade fractions are smaller.

The work on potassium has similarly all been carried out in static vapor experiments rather than with beams.

Volkova (1959) measured excitation functions for two lines of the principal series of neutral potassium and eleven lines of singly ionized potassium excited from the neutral ground state, and measured the absolute values at $60 \mathrm{eV}$. In another paper (Volkova, 1962) are presented absolute values measured at $5 \mathrm{eV}$ for 19 lines of the diffuse and sharp series of neutral potassium. These were all measured using a tungsten ribbon lamp as a radiation standard, and presumably at pressures of about $1.3 \times 10^{-4}$ Torr, although no explicit information

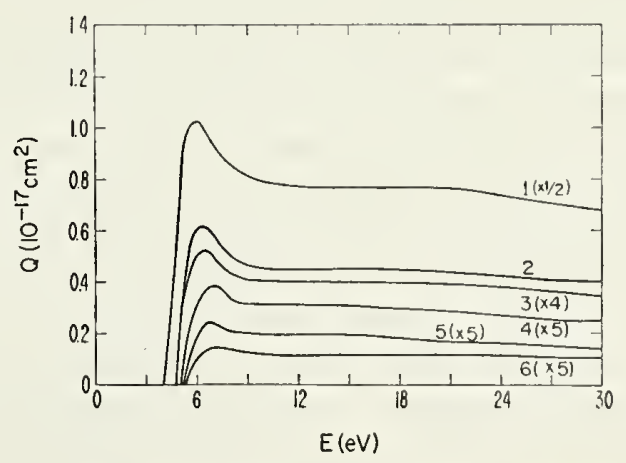

FIG. 97. Absolute excitation functions for the sharp series of

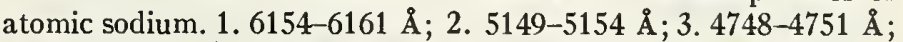
4. 4541-4646 $\AA$; 5. 4420-4423 $\AA$; $4343 \AA$ (Zapesochnyi and Shimon, 1965). 
to this effect is given in the second paper. The shapes of three excitation functions were measured photographically using a retarding field to obtain simultaneous observations over a range of energies as a function of position in the electron beam.

Subsequently, Volkova, and Devyatov (1963) measured the excitation functions of the resonance lines at $7665 \AA$ and $7698 \AA$. This measurement was carried out at a vapor pressure of $2 \times 10^{-6}$ Torr $\left(72^{\circ} \mathrm{C}\right)$, a pressure at which some absorption of resonance radiation occurs. They built a special tube in which the distance from the beam to the window was minimized. The absorption coefficient at the center of the line was calculated and from this the total absorption for a Doppler line was estimated. The assumption of a Doppler line shape was verified by measurement with a Fabry-Perot etalon. The shapes of the functions were also measured at $2 \times 10^{-6}$ Torr. The stated maximum error is $35 \%$.

Another recent study of potassium was reported by Zapesochnyi, Shimon, and Soshnikov (1965). They measured excitation functions of 28 lines of potassium at pressures between $9 \times 10^{-5}$ and $5 \times 10^{-4}$ Torr $\left(122^{\circ}\right.$ $150^{\circ} \mathrm{C}$ ) and made an effort to achieve low electron densities. The static vapor pressure method was used but detection was photoelectric. The error quoted is $30-35 \%$ in the absolute values. These were measured at the two extremes of the static pressure range except for the principal series, and the results differed by $15 \%$ or less.

Excitation functions have been obtained by Zapesochnyi and Shimon (1966a) for cesium, and by Shimon (1964) for rubidium.

Zapesochnyi and Shimon (1966b) designed an apparatus which included a movable window mounted on a bellows, for the purpose of studying the resonance lines of the alkalis. The window was driven by a micrometer screw so that the position, and hence the thickness of absorbing vapor, could be accurately controlled. They showed that the intensity varied exponentially with the thickness of the absorbing layer at sufficiently low pressure and distance in accordance with Beer's law. An extrapolation to zero thickness permits determination of the absolute value. The cross sections for the resonance lines of potassium, rubidium, and cesium obtained in this manner are shown in Figs. 99, 100, and 101. Apparently the method was not applied to sodium, but the results presented here have a similar form to that shown in Fig. 96. Also shown in Fig. 99 are the results obtained by Volkova and Devyatov (1963). Their cross section is peaked near threshold in contrast to the shape obtained by Zapesochnyi and Shimon. The latter authors point out that Volkova and Devyatov used a very high electron beam intensity. In their sodium work Zapesochnyi and Shimon demonstrated that the excitation function was similarly distorted by such dense electron beams.

The absolute values obtained for the resonance lines are shown in Table LXVI.

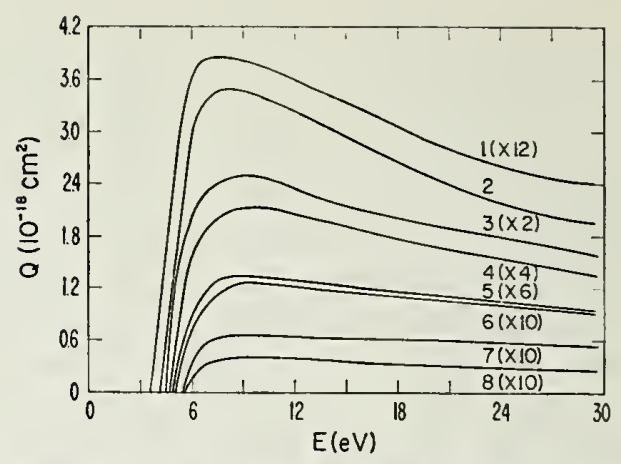

FIG. 98. Absolute excitation functions for the diffuse series of

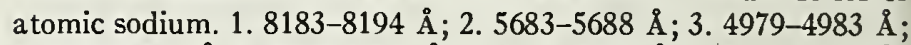

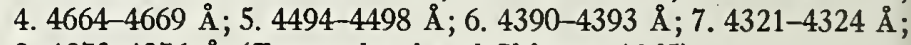
8. 4273-4276 $\AA$ (Zapesochnyi and Shimon, 1965).

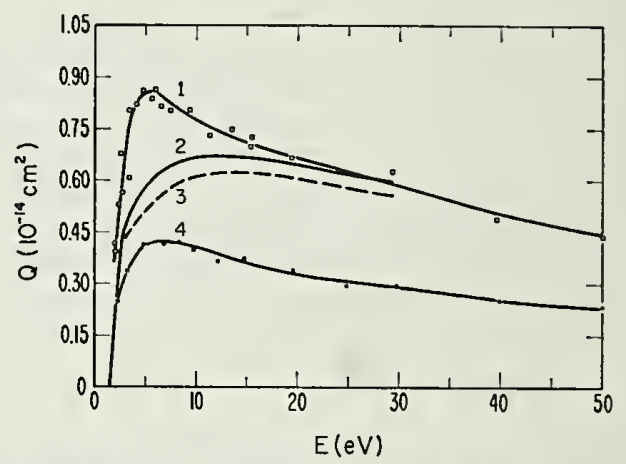

FIG. 99. Absolute excitation functions of the first resonance lines of potassium. Curves 1 and 4 are the excitation functions for the $7665-\AA$ and $7698-\AA$ lines, respectively, measured by Volkova and Devyatov (1963). Curves 2 and 3 are the combined excitation function for the resonance line, with and without cascading, obtained by Zapesochnyi and Shimon (1966b).

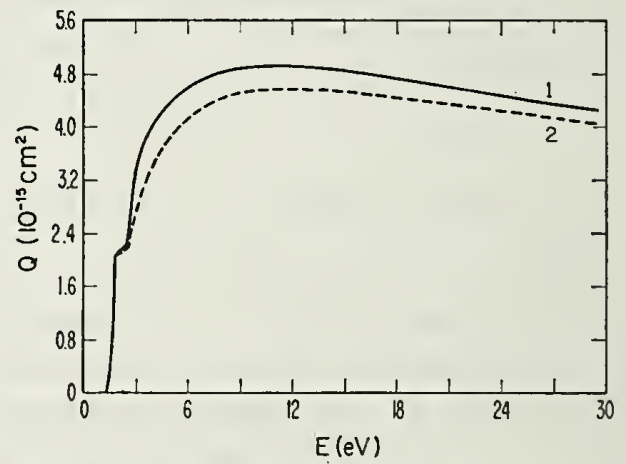

FIG. 100. Absolute excitation function of the rubidium resonance lines (7948-7800 $\AA$ ) with and without cascading (Zapesochnyi and Shimon, 1966b).

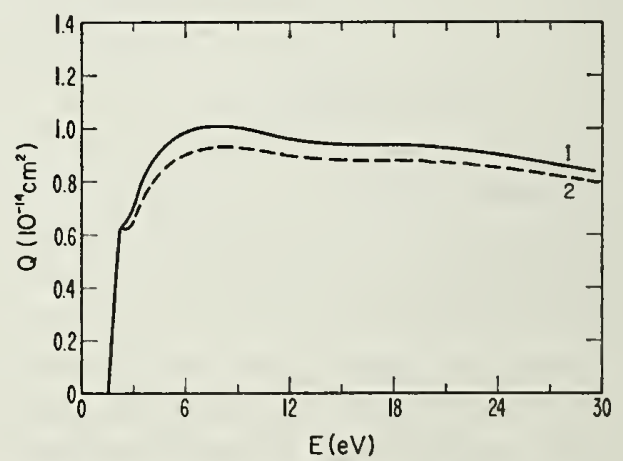

FIG. 101. Absolute excitation function of the cesium resonance lines (8944-8521 $\AA$ ) with and without cascading (Zapesochnyi and Shimon, 1966b). 


\section{Heavy Two-Electron Systems}

The experimental study of helium excitation has been discussed in considerable detail. It is the simplest of the two-electron systems. It has been studied extensively, is relatively tractable theoretically, and has important applications in astrophysics and elsewhere. Of the other two-electron systems, mercury has also been studied extensively and some work has been carried out on cadmium and zinc. These atoms are more complicated, have not received such extensive theoretical treatment, and are not quite so interesting with respect to current applications.

However, they provide some physically interesting contrasts with helium. In helium, $L-S$ coupling holds well and the spectrum is free of inter-system transitions. In the heavier atoms spin-orbit interaction is strong and inter-system transitions occur with competitive probabilities. The energy levels within a given shell are also strongly modified by spin-orbit interaction energy so that there are quite significant differences in energy between states of different angular momenta. This strong spin-orbit interaction, and the fact that the electrostatic interaction with the nucleus is less in the heavier elements, results in a higher lying ground state and larger intervals between some of the relevant upper levels. The transition to the ground state is not so strongly favored over other transitions, energetically, as in helium. One result appears to be that cascade transitions make more significant contributions to the observed excitation functions, as has been demonstrated quite effectively in the more recent experimental work discussed below.

Another result is that trapping of resonance radiation has relatively less influence on the effective lifetime of a given state of the heavy atom than for helium, and therefore less effect on the observed intensity of lines not connecting to the ground state. In helium the $3^{1} P \rightarrow 2^{1} S$ line may be enhanced by a factor of forty through trapping of the $3^{1} P \rightarrow 1^{1} S$ line. The factor is presumably lower for corresponding mercury, cadmium, and zinc lines. The effects of trapping, however important, may be expected to occur at the same pressures, within an order of magnitude, as in the case of helium.

L. J. Kieffer's "Bibliography of Low Energy Electron Collision Cross Section Data" (1967) lists 53 papers under the headings "Electronic Excitation, Experimental, Hg." For helium there are 95, for cadmium 6, and for zinc 3. For the case of helium, there is reason for desiring rather accurate information about the shapes of the excitation functions as well as their absolute values. Considerations of pressure-dependent secondary effects, especially trapping of resonance radiation, have reduced the number of helium papers considered in detail to a relatively small number.

For the case of mercury, the very accurate determination of excitation functions is much less interesting because the theory is not in a refined state and because the applications are not so important. For the case of mercury, zinc, and cadmium, it is the structure of the excitation functions which provides the interest. Thus the essential experimental problems are not so much the refinement of spectroscopic techniques, to which some of the earlier workers may have been more alert than many of the recent workers, but the refinement of photoelectric recording techniques and the techniques of obtaining high-energy resolutions in low-energy electron beams, at which modern workers are beginning to excel. The earlier workers did not expect complex structure and most of them did not see it. Recent work has shown the excitation functions to be rich in structure. Therefore, we will confine our attention to the more recent work. However, we note that authors in this field are either blissfully unaware of, or find it easy to ignore, the distortions which result from polarization through instrumental polarization and as a result of anisotropic radiation patterns.

The pressure dependence problem in mercury seems less serious than in helium, although it should not be ignored to the extent that it is in the literature, particularly where absolute measurements are involved. The pressures used are not always stated explicitly. In most cases the mercury pressures used were probably those of mercury at room temperatures, for which the equilibrium vapor pressure is about $10^{-3}$ Torr, or lower temperatures, for example that of melting ice, for which the pressure is about $2 \times 10^{-4}$ Torr. Zinc and cadmium are used at temperatures of several hundred degrees centigrade in order to achieve comparable vapor pressures.

Evidence that excitation functions of certain lines are highly affected at mercury pressures characteristic of room temperature appear in the literature. Skinner and Appleyard (1927) noted that it was necessary to work at temperatures of $-15^{\circ}$ to $-20^{\circ} \mathrm{C}$ to avoid almost complete depolarization of the $6^{3} P_{1} \rightarrow 6^{1} S_{0}(2537-\AA)$ line which occurs at room temperature. Federov (1965) studied pressure dependence of the $6^{3} D_{2} \rightarrow 6^{1} P_{1}(5770-\AA)$ polarization curve and found that the portion immediately adjacent to threshold where only direct $6^{3} D_{2}$ excitation was effective was decreased by a factor of two in changing the pressure from $8 \times 10^{-4}$ to $10^{-2}$ Torr, and that significant depolarization occurred at $4 \times 10^{-3}$ Torr. His explanation was based on excitation by scattered electrons but at these pressures this does not seem possible. Other secondary processes must be invoked. $\mathrm{He}$ noted the appearance of a halo, reminiscient of the effects observed in helium by Lee and others, and there attributed to trapping of resonance radiation.

The mercury literature contains two papers which should be of special interest to experimentalists working in this or related fields, because they contain unusually complete and interesting accounts of careful experimental investigations. The first of these, by Zapesochnyi (1954), marks the beginning of the modern high resolution work which has revealed the extensive struc- 
turing of the excitation functions. He attributed the structure primarily to cascading. The second paper is a quite comprehensive report on experimental techniques by Jongerius (1961). This paper also includes an excellent summary of the older literature. Both papers describe successful attempts to reproduce results of earlier workers in order to account for the differences. In addition to papers by these workers and their colleagues, one other paper of particular significance by Anderson, Lee, and Lin (1967) has appeared recently. This paper reports a large number of experimental results. It includes only the briefest possible account of experimental techniques, but it contains the most complete analysis and discussion of the structure of the excitation available.

Figure 102 shows the experimental results for the $7^{3} S_{1} \rightarrow 6^{3} P_{2} \quad(5461-\AA)$ line of mercury published by Frish and Zapesochnyi (1956) in support of their original interpretation of the structure in terms of cascading. The lower curves taken from their paper show their interpretation in terms of excitation functions of higher levels. The interpretation is qualitative with respect to shapes and magnitudes but quantitative with respect to intervals between excitation thresholds. It is a quite convincing demonstration, indicating the great importance of cascading in the development of the excitation function. Zapesochnyi (1954) gives similar interpretations of structure for other mercury lines.

It is interesting to note that while most early papers present smooth excitation functions for the 5461- $\AA$ line, Seibertz (1931) succeeded in obtaining most of the essential features in his measurement of the $7^{3} S_{1} \rightarrow$ ${ }^{3} P_{0}$ (4047- $\AA$ ) line for which the excitation function has the same shape as for the 5461- $\AA$ line.

Absolute values of a large number of lines have been obtained by Jongerius (1961) and by Anderson, Lee, and Lin (1967), with a fair degree of consistency between the results. The results by the latter group tend to run higher than those by Jongerius by $15-50 \%$. Older results by Hanle and Schaffernicht (1930) are substantially higher than the recent values.

Of special interest are the relative values of excitation functions with the same upper states. Several such sets have been studied. Jongerius gives relative transition probabilities for lines with $7^{3} S_{1}, 8^{3} S_{1}$, and $6^{3} D_{2}$ upper states. Anderson, Lee, and Lin present results for $7^{3} S_{1}$, ${ }^{1} S_{0}, 8^{1} P_{1}, 9^{1} P_{1}$, and $10^{1} P_{1}$ upper states. These last three involve comparisons between singlet-singlet and singlet-triplet transitions which yield information about the mixing coefficients.

Except for some very early work, the only available data on excitation functions for zinc and cadmium are from recent work by Zapesochnyi and his collaborators. These data reveal structure similar in many respects to that found in mercury. We show on Figs. 103, 104, and 105 some results obtained by Zapesochnyi and Shpenik (1966). This work was accomplished using a $127^{\circ}$ cylindrical electrostatic analyzer to obtain narrow

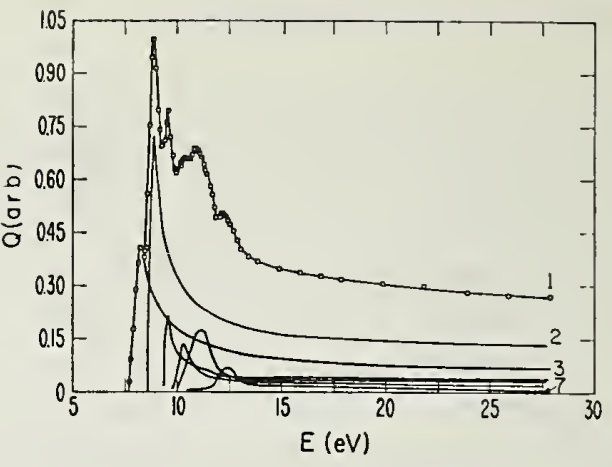

F1G. 102. Relative excitation function for the $7^{3} S_{1} \rightarrow 6^{3} P_{2}$ (5461- $\AA$ ) transition in mercury (Curve 1) showing an interpretation of structure in terms of excitation cross sections for higher levels $\left[(2) 7^{3} P\right.$; (3) $7^{3} S$; (4) $8^{3} P$; (5) $10^{3} P$; (6) $9^{3} P$; (7) ionization plus excitation] by Frish and Zapesochnyi (1956).

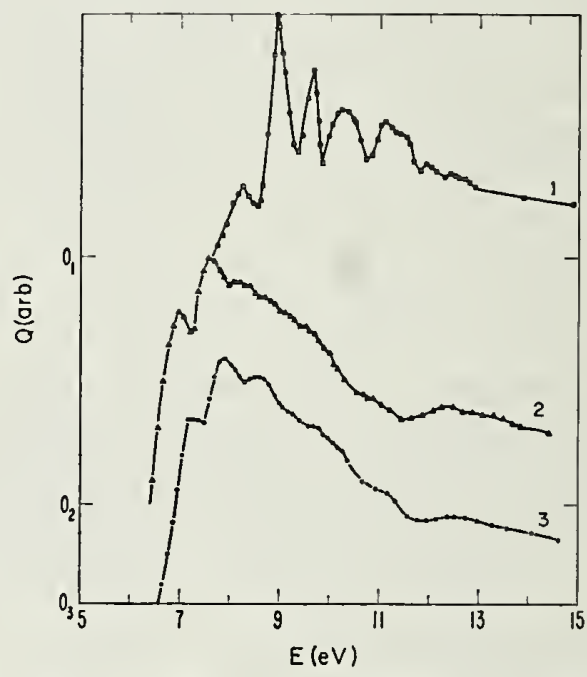

FIG. 103. Relative excitation functions for the corresponding ${ }^{3} S$ states in mercury $\left(7^{3} S_{1}\right)$, Curve 1 ; cadmium $\left(6^{3} S_{1}\right)$, Curve 2 ; and zinc $\left(5^{3} S_{1}\right)$, Curve 3 , measured with an electrostatic electron monochromator to obtain energy spreads of $0.1,0.2$, and $0.3 \mathrm{eV}$, respectively, for the three curves (Zapesochnyi and Shpenik, 1966).

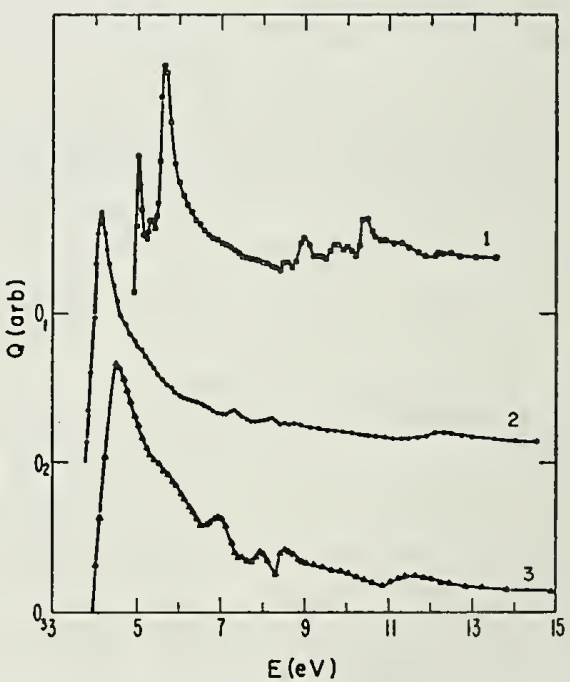

Fig. 104. Relative excitation functions for the corresponding ${ }^{3} P$ states in mercury $\left(6^{3} P_{1}\right)$, Curve 1 ; cadmium $\left(5^{3} P_{1}\right)$, Curve 2 ; and zinc $\left(4^{3} P_{1}\right)$, Curve 3 , measured with an electrostatic electron monochromator (Zapesochnyi and Shpenik, 1966). 


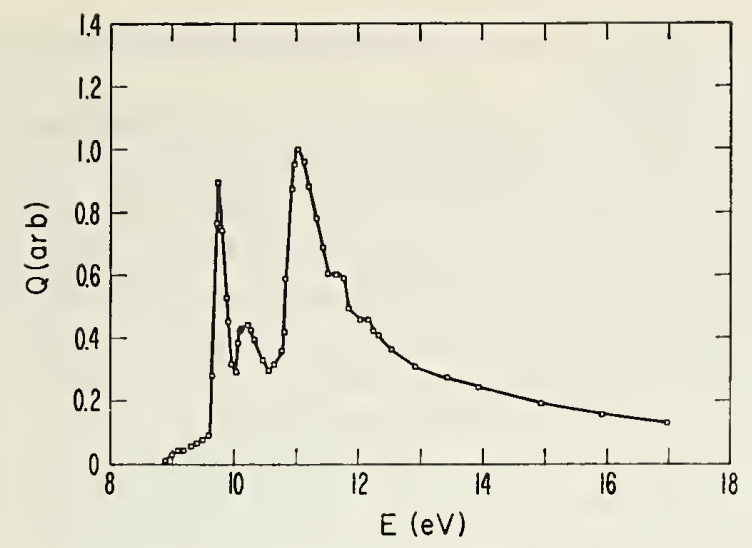

Fig. 105. Highly resolved $(\sim 0.1 \mathrm{eV})$ excitation function for the mercury $3650-\AA$ line originating in the $6^{3} D_{3}$ level (Zapesochnyi and Shpenik, 1966).

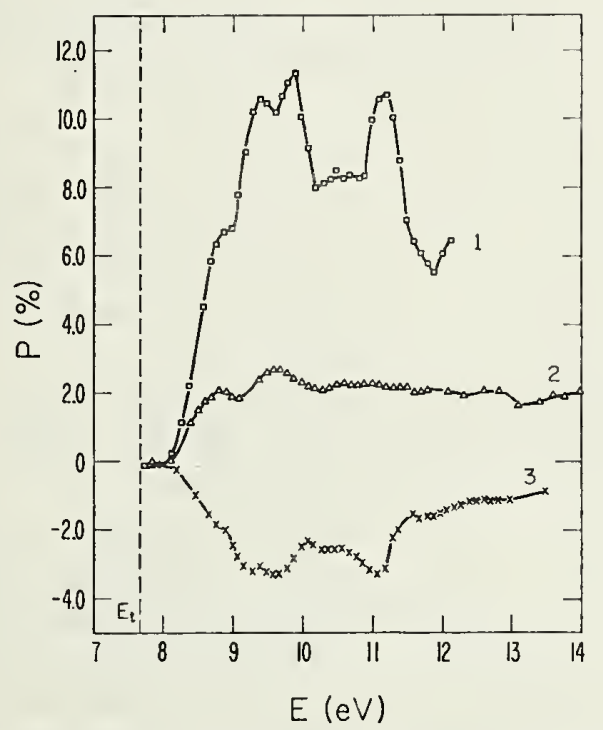

Fig. 106. Polarization of the mercury triplet $7^{3} S_{1} \rightarrow 6^{3} P_{2,1,0}$ as a function of electron energy, measured by Federov and Mezentsev (1965): Curve 1, $4047 \AA$; Curve 2, $5461 \AA$; Curve 3, $4358 \AA$. The occurrence of finite polarization beginning $0.4-0.5 \mathrm{eV}$ above threshold is attributed to cascading from $n^{3} P$ levels.

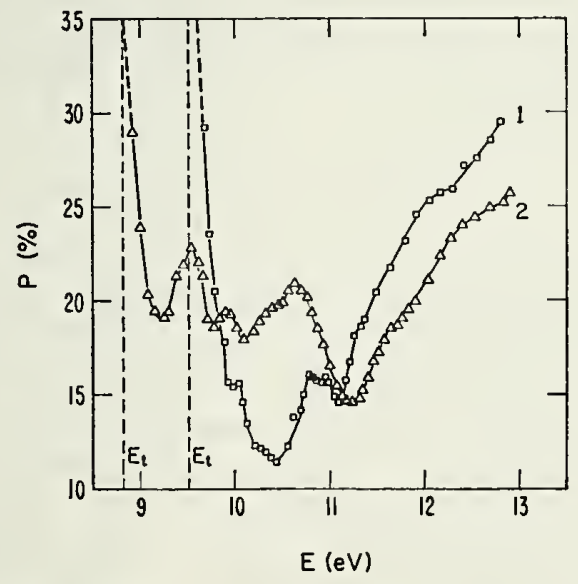

FIG. 107. Polarization of the mercury lines at $4347 \AA\left(7^{1} D_{2} \rightarrow\right.$ $\left.6^{1} P_{1}\right)$, Curve 1 ; and at $5770 \AA\left(6^{3} D_{2} \rightarrow 6^{1} P_{1}\right)$, Curve 2 , as a function of electron energy, measured by Federov and Mezentsev (1965). energy spreads in the electron beam. In Fig. 103 are compared the excitation functions obtained for lines with the upper states $7^{3} S_{1}, 6^{3} S_{1}$, and $5^{3} S_{1}$ in mercury, cadmium, and zinc. The energy spreads used were given as $0.1,0.2$, and $0.3 \mathrm{eV}$ for the three cases. The fact that the mercury structure appears to be narrower is due in part to the energy spread used.

In Fig. 104 are compared the excitation functions corresponding to $6^{3} P_{1}, 5^{3} P_{1}$, and $4^{3} P_{1}$ levels in the three respective atoms, and in Fig. 105 is a highly resolved $6^{3} D_{3}$ excitation for mercury. Zapesochnyi and Shpenik relate the maxima to excitation of higher levels with subsequent cascading.

Skinner and Appleyard (1927) investigated the polarization of a number of the mercury lines excited by electron impact. Their work has been the subject of a great amount of interest because of their observation that the polarization of all lines studied went to zero at threshold. This conflicts with the requirements of conservation of angular momentum at threshold. Another main feature of their results was the highenergy trend to negative polarization of the $6^{1} D_{2} \rightarrow 6^{1} P_{1}$ and ${ }^{1} D_{2} \rightarrow 6^{1} P_{1}$ lines, and the observation of negative maxima in polarization at lower energies for several of their lines.

From the preceding discussion of the importance of cascading in the formation of the mercury excitation functions it is clear that the interpretation of the observed polarization should be quite complex, except near threshold below energies at which the cascading mechanisms can be actuated. Furthermore, one would expect a certain amount of structure in the polarization which does not show up in the work of Skinner and Appleyard. Federov and Mezentsev (1965) have recently studied the polarization of some mercury lines in the region near threshold. Using relatively narrow energy spreads $(\sim 0.6 \mathrm{eV})$ they find structure in the polarization curves related to cascading. They find the lines ${ }^{1} S_{0} \rightarrow 6^{1} P_{1}$ and $7^{1} S_{0} \rightarrow 6^{3} P_{1}$ unpolarized near threshold.

Their polarization curves for the $7^{3} S_{1} \rightarrow 6^{3} P_{2,1,0}$ lines are shown in Fig. 106. The polarization is zero near threshold but becomes finite half a volt above threshold because of cascading from the $n^{3} P$ levels. In Fig. 107 are shown the polarization curves for the $7^{1} D_{2} \rightarrow 6^{1} P_{1}$ $(4347-\AA)$ and $6^{3} D_{2} \rightarrow 6^{1} P_{1}(5770-\AA)$ transitions near threshold. Skinner and Appleyard found the 4347polarization to fall to zero near threshold, but with better resolution Federov and Mezentsev show that it rises again as threshold is approached from a higher energy, in a manner not inconsistent with the requirements of angular momentum conservation. The behavior is similar to that now found in helium.

\section{Experimental Summary}

Experimental research in the electron impact excitation of atoms is entering a new phase. Many of the 
important atomic excitation functions have been surveyed again and again in semi-quantitative fashion. There is little point to more work of this type on helium. The technology is available to support reliable measurements of relative excitation functions. The technology must be applied with due attention to the physical properties of the atom under study and to the effects of ambient fields on the observations. Hopefully, in this article we have indicated the important physical properties and processes to be considered, at least for helium. For atoms heavier than helium much more study is needed in order to determine the nature and importance of secondary processes.

The quality of absolute measurement depends on the quality of radiometric and gas density standards, and on the convenience with which they can be applied in the laboratory. At the present time the reliability of absolute measurement cannot be much better than $10 \%$. The reproducibility with which the standards are applied from one laboratory to another is reflected in the scatter of absolute measurements of a given excitation function at the same energy, as in Tables LXIVd and LXIVe. The scatter does not provide an indication of the true reliability of the measured values, since certain systematic effects may occur in all the measurements and since the standards tend to be coupled through intercomparison and through use of common supporting data. The significance which may be attached to a comparison between theory and experiment is limited accordingly. Other limitations include the uncertainty in making proper allowance for cascading, polarization, and imprisonment of resonance radiation.

Some indication of the significance of a shape comparison between experiment and theory can be gained from considering the figures in which we have intercompared the experimentally determined helium excitation functions. Some authors of the experimental results have clearly been more thorough in their treatment of secondary and instrumental effects than others. However, more high quality work is necessary before a comparison between theoretical and experimental shapes could be trusted in great detail. One serious limitation clearly lies in the current confusion over the polarization of radiation excited by electron impact, apparent in Fig. 73; but there are other limitations as may be seen from consideration of Fig. 83 for the $4^{3} S$ excitation function and of Fig. 79 for the $4^{1} S$ excitation function, for which polarization should not be a factor. Accurate allowance for cascading may be a serious limitation in these and other cases.

However, the comparison between theory and experiment has provided some extremely important results:

(1) In the range of energies below 50 or $60 \mathrm{eV}$ the close coupling calculations, involving as many states as it is presently practical to include but omitting higher states and the continuum, yield results which rise much higher than can be considered compatible with experimental results,

(2) except in the immediate vicinity of threshold where all open channels can be included in the close coupling calculation. In the threshold case there has been some remarkable agreement between theory and experiment, as is evident in Figs. 65, 67, and 88.

(3) The comparison between theory and experiment for the helium triplet excitation functions shows that there is a serious discrepancy in the high-energy behavior, the theoretical results falling off as $E^{-3}$ and the experimental results falling much more slowly.

If it is difficult to make definitive statements about the quality of helium excitation functions, it is even more difficult for the excitation functions of the heavier atoms. The study of helium excitation gives a picture of secondary and instrumental effects which must apply to work on other atoms. However, there is little evidence in the literature that secondary effects for heavier atoms have been properly considered, and it must be presumed that the available data contains serious distortions.

The future of electron impact excitation work may lie in the further development of methods of measuring the inelastically scattered electron currents. This would require measurement of scattered currents at all angles and subsequent integration over the total solid angle. It is not clear that accuracy can be achieved at the lower electron energies. Nevertheless, the method is attractive because it would yield direct excitation cross sections. There would be no cascade problem, no imprisonment problem, and no collisional excitation transfer problem. The angular distribution of scattered electrons would certainly be nonisotropic, which would present a problem fully as difficult as that presented by the polarization of the radiation in the optical method.

However, the only one of the above problems which cannot be avoided in the optical method is that of cascading. It seems that the optical method has not yet been properly exploited. With a proper consideration of the physics involved, more carefully planned experimental work, and more adequate treatment in publishing results, the full value of the optical method could be realized.

\section{ACKNOWLEDGMENTS}

This review was supported by the National Bureau of Standards through the National Standard Reference Data Program. The authors are grateful for the use of facilities of the JILA Information Center, which is supported by the Advanced Research Projects Agency (Project DEFENDER), which is monitored by the U.S. Army Research Office-Durham, and the National Bureau of Standards, through the National Standards Reference Data Program. We are also grateful for the many months of conscientious and careful work by the Information Center staff, particularly by Elizabeth Hosack, Mary Ann McCormick, and Ross Fraker, on 
the preparation of data, figures, and references for this article.

We would like to express our thanks to M. J. Seaton, D. W. O. Heddle, and L. J. Kieffer for very helpful discussions of some aspects of this review. One of us, S. J. Smith, would like to acknowledge the contribution made by his scientific colleagues in JILA through innumerable discussions of relevant experimental problems.

\section{BIBLIOGRAPHY}

Altshuler, S., 1952, Phys. Rev. 87, 992.

,- 1953, Phys. Rev. 89, 1093.

Anderson, R. J., E. T. P. Lee, and C. C. Lin, 1967, Phys. Rev. 157,31 .

Barnes, L. L., N. F. Lane, and C. C. Lin, 1965, Phys. Rev. 137, A388.

Bates, D. R., A. Fundaminsky, J. W. Leech, and H. S. W. Massey, 1950, Phil. Trans. Roy. Soc. London A243, 93.

— Becker, P. M., and J. S. Dahler, 1964, Phys. Rev. 136, A73. Bell, K. L., 1965, Proc. Phys. Soc. (London) 86, 246.

-, H. Eissa, and B. L. Moiseiwitsch, 1966, Proc. Phys. Soc. (London) 88, 57.

, and B. L. Moiseiwitsch, 1963, Proc. Roy. Soc. (London) A276, 346.

Bely, O., 1962, Compî. Rend. 254, 3075.

- , J. Tully, and H. Van Regemorter, 1963, Ann. Phys. (Paris) $8,303$.

Bethe, H., and E. E. Salpeter, 1957, Quantum Mechanics of One and Two Electron Systems (Academic Press Inc., New York).

Biberman, L. M., 1947, Zh. Eksperim. i Teor. Fiz. 17, 416.

Bogdanova, I. P., O. P. Bochkova, and S. E. Frish, 1964, Dokl. Akad. Nauk SSSR 156, 54 [English transl.: Soviet Phys.Doklady 9, 370 (1964)].

, and I. I. Geitsi, 1964, Opt. i Spektroskopiya 17, 151 [English transl.: Opt. Spectry. USSR 17, 80 (1964) ].

- and V. D. Marusin, 1966, Opt. i Spektroskopiya 20, 209 [English transl.: Opt. Spectry. USSR 20, 113 (1966)].

Boikova, R. F., and E. E. Fradkin, 1965, Opt. i Spektroskopiya 19, 843 [English transl.: Opt. Spectry. USSR 19, 470 (1965)]. Boyd, T. J. M., 1959, Proc. Phys. Soc. (London) 72, 523.

Bransden, B. H., A. Dalgarno, and N. M. King, 1953, Proc. Phys. Soc. (London) A66, 1097.

- , and J. S. C. McKee, 1956, Proc. Phys. Soc. (London) A69, 422.

Burgess, A., 1961, Mem. Soc. Roy. Sci. Liege 4, 299.

$\longrightarrow, 1964$, in Atomic Collision Processes, M. R. C. McDowell, Ed. (North-Holland Publ. Co., Amsterdam), p. 237. [Proceedings of the Third International Conference on the Physics of Electronic and Atomic Collisions (London, 22-26 July 1963)].

- D. D. Hummer, and J. A. Tully, 1961, in Abstracts of the Second International Conference on the Physics of Electronic and Atomic Collisions (Boulder, 12-15 June 1961) (W. A. Benjamin, Inc., New York), p. 173.

, and I. C. Percival, 1968, to be published in Advances in Atomic and Molecular Physics, D. R. Bates and I. Estermann, Eds. (Academic Press Inc., New York), Vol. 4.

Burke, P. G., 1963, Proc. Phys. Soc. (London) 82, 443.

- , V. M. Burke, I. C. Percival, and R. McCarroll, 1962, Proc. Phys. Soc. (London) $80,413$.

- J. W. Cooper, and S. Ormonde, 1966, Phys. Rev. Letters 17,345 .

, D. D. McVicar, and K. Smith, 1964a, Proc. Phys. Soc. (London) 83, 397.

, D. D. McVicar, and K. Smith, 1964b, Proc. Phys. Soc. (London) 84, 749.

- , S. Ormonde, A. J. Taylor, and W. Whitaker, 1967, in abstracts of, The Fifth International Conference on the Physics of
Electronic and Atomic Collisions (Leningrad, 17-23 July 1967), p. 368.

, S. Ormonde, and W. Whitaker, 1966, Phys. Rev. Letters 17, 800.

—_, 1967, Proc. Phys. Soc. (London) 92, 319.

- A. J. Taylor, and S. Ormonde, 1967, Proc. Phys. Soc. (London) 92,345 .

- - H. M. Schey, and K. Smith, 1963, Phys. Rev. 129, 1258.

, J. H. Tait, and B. A. Lewis, 1966, Proc. Phys. Soc. London $87,209$.

- and A. J. Taylor, 1966, Proc. Phys. Soc. (London) 88, 549.

- , and R. McCarroll, 1962, Proc. Phys. Soc. (London) 80, 422.

__, and M. J. Seaton, 1961, Proc. Phys. Soc. (London) 77, 199.

Cêrmak, V., 1966, J. Chem. Phys. 44, 3774.

Chamberlain, G. E., 1965, Phys. Rev. Letters 14, 581.

- , and H. G. M. Heideman, 1965, Phys. Rev. Letters 15, 337.

- - , S. J. Smith, and D. W. O. Heddle, 1964, Phys. Rev. Letters 12,647 .

- J. A. Simpson, and C. E. Kuyatt, 1965, in abstracts of The Fourth International Conference on the Physics of Electronic and Atomic Collisions (Quebec, 2-6 August 1965) (Science

Bookcrafters, Inc., Hastings-on-Hudson, New York), p. 378. Christoph, W., 1935, Ann. Physik 23, 51.

Clementi, E., C. C. J. Roothaan, and M. Yoshimine, 1962, Phys. Rev. 127, 1618.

Corinaldesi, E., and L. Trainor, 1952, Nuovo Cimento 9, 940. Crothers, D., 1967, Proc. Phys. Soc. (London) 91, 855.

—- and R. McCarroll, 1965, Proc. Phys. Soc. (London) 86, 753. Czysak, S. J., and T. K. Krueger, 1964, in Atomic Collision Processes, M. R. C. McDowel, Ed. (North-Holland Publ. Co., Amsterdam), p. 213. [Proceedings of the Third International Conference on the Physics of Electronic and Atomic Collisions (London, 22-26 July 1963) ].

623.

Damburg, R. Ya., 1962, Opt. i Spektroskopiya 12, 787 [English transl.: Opt. Spectry. USSR 12, 445 (1962)].

- and M. Gailitis, 1963, Proc. Phys. Soc. (London) 82, 1068.

, and R. K. Peterkop, 1962a, Opt. i Spektroskopiya 13, 597

[English transl.: Opt. Spectry. USSR 13, 338 (1962)].

_- and R. K. Peterkop, 1962b, Proc. Phys. Soc. (London) 80,563 .

— 1073.

_- and R. K. Peterkop, 1963, Zh. Eksperim. i Teor. Fiz. 43, 1765 [English transl.: Soviet Phys.-JETP 16, 1247 (1963) ].

Dance, D. F., M. F. A. Harrison, and A. C. H. Smith, 1966, Proc. Roy. Soc. (London) A290, 74.

Devyatov, A. M., and H. A. Kaptsov, 1955, Vest. Mosk. Univ. Ser. Fiz. Mat. Estestven. Nauk 10, 27.

Dolgov, G., 1959, Opt. i Spektroskopiya 6, 717 [English transl.: Opt. Spectry. USSR 6, 469 (1959)].

Dorrestein, R., 1942, Physica 9, 447.

Drukarev, G. F., 1953, Zh. Eksperim. i Teor. Fiz. 25, 139.

Dugan, J. L. G., H. L. Richards, and E. E. Muschlitz, 1967, J. Chem. Phys. 46, 346.

Eckhart, C., 1930, Phys. Rev. 36, 878.

Erskine, G. A., and H. S. W. Massey, 1952, Proc. Roy. Soc. (London) A212, 521.

Federov, V. L., 1965, Opt. i Spektroskopiya 19, 147 [English transl.: Opt. Spectry. USSR 19, 79 (1965)].

, and A. P. Mezentsev, 1965, Opt. i Spektroskopiya 19, 12 [English transl.: Opt. Spectry. USSR 19, 5 (1965)].

Feenberg, E., 1932, Phys. Rev. 40, 40.

Feldman, P., and R. Novick, 1963, Phys. Rev. Letters 11, 278. Fischer, O., 1933, Z. Physik 86, 646.

Fisher, L., S. N. Milford, and F. R. Pomilla, 1960, Phys. Rev. $119,153$.

Fite, W. L., 1962, in Atomic and Molecular Processes, D. R. Bates, Ed. (Academic Press Inc., New York), p. 421.

-_, and R. T. Brackmann, 1958, Phys. Rev. 112, 1151. 
- , R. F. Stebbings, and R. T. Brackmann, 1959, Phys. Rev. 116,356 .

Fleming, R. J., and G. S. Higginson, 1964, Proc. Phys. Soc. (London) 84,531 .

Flower, D. R., and M. J. Seaton, 1967, Proc. Phys. Soc. (London) $91,59$.

Fox, M. A., 1965, Proc. Phys. Soc. (London) 86, 789.

—_, 1966, Proc. Phys. Soc. (London) 88, 65.

__ 1967, Erratum, Proc. Phys. Soc. (London) 90, 585.

Frish, S. E., and I. P. Zapesochnyi, 1956, Izv. Akad. Nauk SSSR Ser. Fiz. 19, 5 [English transl.: Bull. Acad. Sci. USSR Phys. Ser. 19, 1 (1956)].

Gabriel, A. H., and D. W. O. Heddle, 1960, Proc. Roy. Soc. (London) A258, 124.

Gailitis, M., 1963, Opt. i Spektroskopiya 14 , 465 [English transl.: Opt. Spectry. USSR 14, 249 (1963)].

—_, and R. Ya. Damburg, 1963, Proc. Phys. Soc. (London) 82, 192.

Gaspar, R., 1952, Acta Phys. Acad. Sci. Hungary 2, 151.

Goldberg, L., and A. M. Clogston, 1939, Phys. Rev. 56, 696.

Green, L. C., M. M. Mulder, M. N. Lewis, and J. W. Woll, 1954, Phys. Rev. 93, 757.

Gryzinski, M., 1959, Phys. Rev. 115, 374.

- 1965a, Phys. Rev. 138, A305.

- 1965 b, Phys. Rev. 138, A322.

—., 1965c, Phys. Rev. 138, A336.

Guk, Sin San, and A. M. Devyatov, 1957, Vest. Mosk. Univ. 1, 63.

Hafner, H., and H. Kleinpoppen, 1967, Z. Physik 198, 315.

,- H. Kleinpoppen, and H. Kruger, 1965, Phys. Letters 18, 270.

Haft, G., 1933, Z. Physik 82, 73.

Hanle, W., 1930, Z. Physik 65, 512.

- and W. Shaffernicht, 1930, Ann. Physik 6, 905.

Hansen, L. K., 1964, J. Appl. Phys. 35, 254.

Heath, D. F., and P. A. Sacker, 1966, Appl. Opt. 5, 937.

Heddle, D. W. O., 1967, Proc. Phys. Soc. (London) 90, 81.

- in press, "The Measurement of Optical Excitation Functions," to be published in Methods of Experimental Physics, L. Marton, Ed. (Academic Press Inc., New York), Vol. 8.

- , and R. G. W. Keesing, 1964, in Atomic Collision Processes, M. R. C. McDowell, Ed. (North-Holland Publ. Co., Amsterdam), p. 179. [Proceedings of the Third International Conference on the Physics of Electronic and Atomic Collisions (London, 22-26 July 1963)].

- - and R. G. W. Keesing, 1965, in Abstracts of The Fourth International Conference on the Physics of Electronic and Atomic Collisions (Quebec, 2-6 August 1965) (Science Bookcrafters, Inc., Hastings-on-Hudson, New York), p. 382.

- and R. G. W. Keesing, 1967a, Proc. Phys. Soc. (London) 91 , 510.

- and R. G. W. Keesing, 1967b, Proc. Roy. Soc. (London) A299, 212.

- - and R. G. W. Keesing, 1968, "Measurements of Electron Excitation Functions," to be published in Advances in Atomic and Molecular Physics, D. R. Bates and I. Estermann Eds (Academic Press Inc., New York), Vol. 4.

- and C. B. Lucas, 1961, in Abstracts of The Secosid International Conference on the Physics of Electronic and Alomic Collisions (Boulder, 12-15 June 1961) (W. A. Benjamin, Inc., New York), p. 119.

_- and C. B. Lucas, 1963, Proc. Roy. Soc. (London) A271, 129.

Herrmann, O., 1936, Ann. Physik 25, 143.

Hils, D., H. Kleinpoppen, and H. Koschmeider, 1966, Proc. Phys. Soc. (London) 89, 35.

Holstein, T., 1947, Phys. Rev. 72, 1212.

$\longrightarrow, 1951$, Phys. Rev. 83, 1159.

Holt, A. R., and B. L. Moiseiwitsch, 1968, Proc. Phys. Soc. (London) $\mathbf{B 1}, 36$.

Holt, H. K., and R. Krotkov, 1966, Phys. Rev. 144, 82.

Houston, W. V., 1929, Phys. Rev. 33, 297.

Hughes, R. H., and G. G. Hendrickson, 1964, J. Opt. Soc. Am. 54,1494 .
- , R. B. Kay, and L. D. Weaver, 1963, Phys. Rev. 129, 1630. Hultgren, R., R. L. Orr, P. D. Anderson, and K. K. Kelley, 1963, Selected Values of Thermodynamic Properties of Metals and Alloys (John Wiley \& Sons, Inc., New York).

Hylleraas, E. A., 1929, Z. Physik 54, 347.

,- 1930 , Ann. Physik 65, 209.

Inokuti, M., 1967, J. Phys. Soc. Japan, 22, 971.

Ivanova, A. V., and A. N. Ivanova, 1964, Opt. i Spektroskopiya 16, 917 [English transl.: Opt. Spectry. USSR 16, 499 (1964)].

Joachain, C. G., and M. J. Mittleman, 1965, Phys. Rev. 140, A432.

Jongerius, H. M., 1961, thesis, University of Utrecht.

Karule, E. M., and R. K. Peterkop, 1964, Opt. i Spektroskopiya 16, 958 [English transl.: Opt. Spectry. USSR 16, 519 (1964)]. - , and R. K. Peterkop, 1965, in Atomic Collisions III, Akad. Nauk Latv. SSR Inst. Fiz., V. Ya. Veldre, Ed., Riga, p. 3.

[Translation TT-66-12939 available through SLA Translation Center, John Crerar Library, Chicago, p. 1].

Kay, R. B., and R. H. Hughes, 1967, Phys. Rev. 154, 61.

Khashaba, S., and H. S. W. Massey, 1958, Proc. Phys. Soc. London 71, 574.

Kieffer, L. J., 1967, National Bureau of Standards Miscellaneous Publication 289.

—. , and G. H. Dunn, 1966, Rev. Mod. Phys. 38, 1.

Kingston, A. E., 1964, Phys. Rev. 135, A1529.

-_, and J. E. Lauer, 1966a, Proc. Phys. Soc. (London) 87, 399.

—, and J. E. Lauer, 1966b, Proc. Phys. Soc. (London) 88, 597.

- B. L. Moiseiwitsch, and B. G. Skinner, 1960, Proc. Roy. Soc. (London) A258, 245.

Kleinpoppen, H., and E. Kraiss, 1967, Phys. Rev. Letters 20, 361. Kostkowski, H. J., and R. D. Lee, 1962, National Bureau of Standards Monograph No. 41.

Krueger, T. K., and S. J. Czyzak, 1965, Mem. Roy. Astron. Soc. $69,145$.

Lamb, W. E., and T. H. Maiman, 1957, Phys. Rev. 105, 573. Lane, N. F., and C. C. Lin, 1964, Phys. Rev. 133, A947.

Lashmore-Davies, C. N., 1965, Proc. Phys. Soc. (London) 86, 783.

Lassettre, E. N., and E. A. Jones, 1964, J. Chem. Phys. 40, 1218. $\longrightarrow$, M. E. Krasnow, and S. Silverman, 1964, J. Chem. Phys. $40,1242$.

Lawson, J., W. Lawson, and M. J. Seaton, 1961, Proc. Phys. Soc. (London) 77, 192.

Lees, J. H., 1932, Proc. Roy. Soc. (London) A137, 173.

- , and H. W. B. Skinner, 1932, Proc. Roy. Soc. (London) A137, 186.

Lichten, W., and S. Schultz, 1959, Phys. Rev. 116, 1132.

Lin, C. C., and R. G. Fowler, 1961, Ann. Phys. (N.Y.) 15, 561. $\longrightarrow$, and R. M. St. John, 1962, Phys. Rev. 128, 1749.

Long, R. L., D. M. Cox, and S. J. Smith, to be submitted for publication in J. Res. Natl. Bur. Std.

Madden, R. P., and K. Codling, 1963, Phys. Rev. Letters 10, 516; Proceedings of the Sixth International Conference on Ionization Phenomena in Gases (Paris, 8-13 July 1963), P. Hubert and E. Cremieu-Alcan, Eds. (Serma, Paris), Vol. 1, p. 139.

Maeir-Leibnitz, H., 1935, Z. Physik 95, 499.

Marriott, R., 1957, Proc. Phys. Soc. (London) A70, 288.

,- 1958, Proc. Phys. Soc. (London) 72, 121.

_- 1964, in Atomic Collision Processes, M. R. C. McDowell, Ed. (North-Holland Publ. Co., Amsterdam), p. 114. [Proceedings of the Third International Conference on the Physics of Electronic and Atomic Collisions (London, 22-26 July 1963)]. -, 1966, Proc. Phys. Soc. (London) 87, 407.

$\longrightarrow$, and M. J. Seaton, 1957, Proc. Phys. Soc. (London) A70, 296.

Massey, H. S. W., 1956, in Handbuch der Physik, S. Flügge, Ed. (Springer-Verlag, Berlin), Vol. 36/II, p. 307.

-, and C. B. O. Mohr, 1931, Proc. Roy. Soc. (London) Al32, 605.

187, and C. B. O. Mohr, 1933a, Proc. Roy. Soc. (London) A139, 187. 
, and C. B. O. Mohr, 1933b, Proc. Roy. Soc. (London) A140, 613.

, and C. B. O. Mohr, 1934, Proc. Roy. Soc. (London) A146, 880.

- , and C. B. O. Mohr, 1935, Proc. Cambridge Phil. Soc. 31, 604.

, and B. L. Moiseiwitsch, 1954, Proc. Roy. Soc. (London) A227, 38.

- and B. L. Moiseiwitsch, 1960, Proc. Roy. Soc. (London) A258, 147.

McCarroll, R., 1957, Proc. Phys. Soc. (London) A70, 460.

- 1964, Proc. Phys. Soc. (London) 83, 409.

McConnell, J. C., and B. L. Moiseiwitsch, 1968, to be published in Proc. Phys. Soc. (London).

McCoyd, G. C., and S. N. Milford, 1963, Phys. Rev. 130, 206.

- , S. N. Milford, and J. J. Wahl, 1960, Phys. Rev. 119, 149.

McCrea, D., and T. V. M. McKirgan, 1960, Proc. Phys. Soc.

(London) 75, 235.

McFarland, R. H., 1963, Phys. Rev. Letters 10, 397.

-, 1964, Phys. Rev. 133, A986.

, 1967, Phys. Rev. 156, 55.

_, and E. A. Soltysik, 1961, Bull. Am. Phys. Soc. 6, 423.

, and E. A. Soltysik, 1962, Phys. Rev. 127, 2090.

Milatz, J. M. W., and L. S. Ornstein, 1935, Physica 2, 355.

_- and J. P. M. Woudenberg, 1940, Physica 7, 697.

Milford, S. N., 1960, Astrophys. J. 131, 407.

- J. J. Morrissey, and J. H. Scanlon, 1960, Phys. Rev. 120, 1715.

Miller, F. L., 1964, thesis, University of Oklahoma.

Miller, W. F., and R. L. Platzman, 1957, Proc. Phys. Soc. (London) A70, 299.

Moiseiwitsch, B. L., 1957, Monthly Notices Roy. Astron. Soc. $117,189$.

, and R. Perrin, 1965, Proc. Phys. Soc. (London) 85, 5.1.

Morse, P. M., L. A. Young, and E. S. Haurwitz, 1935, Phys. Rev. 48, 948.

Mott, N. F., and H. S. W. Massey, 1965a, Theory of Atomic Collisions (Clarendon Press, Oxford, England), 3rd ed, Chap., XII, p. 324.

- , and H. S. W. Massey, 1965b, Theory of Atomic Collisions (Clarendon Press, Oxford, England), 3rd ed., Chap. XV, p. 388.

Moustafa Moussa, H. R., 1967, thesis, Leiden.

Ochkur, V. I., 1958, Vest. Leningr. Univ. Ser. Fiz. i Khim. 4, 53. - 1963, Zh. Eksperim. i Teor. Fiz. 45, 734 [English transl.: Soviet Phys.-JETP 18, 503 (1964)].

- , and V. F. Bratsev, 1965a, Astron. Zh. 42, 1035 [English transl.: Soviet Astron.-AJ 9, 797 (1966) ].

- , and V. F. Bratsev, 1965b, Opt. i Spektroskopiya 19, 490

[English transl.: Opt. Spectry. USSR 19, 274 (1965)].

Omidvar, K., 1964 NASA Technical Note TN-D-2145. , 1965, Phys. Rev. 140, A38.

, 1967, Phys. Rev. Letters 18, 153.

Oppenheimer, J. R., 1927a, Proc. Natl. Acad. Sci. (U.S.) 13, 800. , 1927b, Z. Physik 43, 27.

, 1928, Phys. Rev. 32, 361.

Ornstein, L. S., and H. Lindeman, 1933, Z. Physik 80, 525.

Ott, W. R., W. E. Kauppila, and W. L. Fite, 1967, in abstracts of

The Fifth International Conference on the Physics of Electronic and Atomic Collisions (Leningrad, 17-23 July 1967), p. 534.

Penney, W. G., 1932a, Phys. Rev. 39, 467.

-, 1932b, Proc. Natl. Acad. Sci. (U.S.) 18, 231.

Percival, I. C., 1957, Proc. Phys. Soc. (London) A70, 241.

, and M. J. Seaton, 1957, Proc. Cambridge Phil. Soc. 53, 654.

-, and M. J. Seaton, 1958, Phil. Trans. Roy. Soc. (London)

A251, 113.

Peterkop, R., and V. Ya. Veldre, 1966, in Advances in Atomic and

Molecular Physics, D. R. Bates and I. Esterman, Eds. (Aca-

demic Press Inc., New York), Vol. 2, p. 264.

Phelps, A. V., 1955, Phys. Rev. 99, 1307. , 1958, Phys. Rev. 110, 1362.
Purcell, E. M., 1952, Astrophys. J. 116, 457.

Rothenstein, W., 1954, Proc. Phys. Soc. (London) A67, 673.

Ruark, A. E., and H. C. Urey, 1930, Atoms, Molecules and Quanta (McGraw-Hill Book Co., Inc., New York).

Rudge, M. R. H., 1965, Proc. Phys. Soc. (London) 86, 763.

St. John, R. M., C. J. Bronco, and R. G. Fowler, 1960, J. Opt. Soc. Am. 50, 28.

- and R. G. Fowler, 1961, Phys. Rev. 122, 1813.

- - and J. D. Jobe, 1967, in abstracts of The Fifth International Conference on the Physics of Electronic and Atomic Collisions (Leningrad, 17-23 July 1967), p. 517.

— , F. L. Miller, and C. C. Lin, 1964, Phys. Rev. 134, A888.

Salmona, A., and M. J. Seaton, 1961, Proc. Phys. Soc. (London) $77,617$.

Saraph, H. E., 1964, Proc. Phys. Soc. (London) 83, 763.

(London) 89, 27.

Scanlon, J. H., and S. N. Milford, 1961, Astrophys. J. 134, 724.

Schulz, G. J., and R. E. Fox, 1957, Phys. Rev. 106, 1179.

-, and J. Philbrick, 1964, Phys. Rev. Letters 13, 477.

Seaton, M. J., 1953a, Phil. Trans. Roy. Soc. London A245, 469.

-, 1953 b, Proc. Roy. Soc. (London) A218, 400.

_- 1955a, Proc. Phys. Soc. (London) 68, 457.

—_, 1955b, Proc. Roy. Soc. (London) A231, 37.

- 1956, The Airglow and the Aurorae (Pergamon Press, London), p. 289.

—, 1961, Proc. Phys. Soc. (London) 77, 174.

_-, 1962, Proc. Phys. Soc. (London) 79, 1105.

Shimon, L. L., 1964, Opt. i Spektroskopiya 17 , 24 [English transl.:

Opt. Spectry. USSR 17, 12 (1964)].

Siebertz, K., 1931, Z. Physik 68, 505.

Silverman, S. M., and E. N. Lassettre, 1964, J. Chem. Phys. 40, 1265.

Simpson, J. A., S. R. Mielczarek, and R. Cooper, 1964, J. Opt. Soc. Am. 54, 269.

Skinner, H. B. W., and E. T. S. Appleyard, 1928, Proc. Roy. Soc. (London) A117, 224.

Smit, C., H. G. M. Heideman, and J. A. Smit, 1963, Physica 29, 245.

Smith, K., 1960, Phys. Rev. 120, 845.

- R. J. W. Henry, and P. G. Burke, 1966, Phys. Rev. 147, 21.

- - W. F. Miller, and A. J. P. Mumford, 1960, Proc. Phys. Soc. (London) 76, 559.

Smith, S. J., 1965, in Abstracts of The Fourth International Conference on the Physics of Electronic and Atomic Collisions (Quebec, 2-6 August 1965) (Science Bookcrafters, Inc., Hastings-on-Hudson, New York), p. 377.

Soltysik, E. A., A. Y. Fournier, and R. L. Gray, 1967, Phys. Rev. 153, 152.

Somerville, W. B., 1962, Proc. Phys. Soc. (London) 80, 806.

- 1963 , Proc. Phys. Soc. (London) 82, 446.

Stabler, R. C., 1964, Phys. Rev. 133, A1268.

Stebbings, R. F., 1957, Proc. Roy. Soc. (London) A241, 270.

- W. W. Fite, D. G. Hummer, and R. T. Brackmann, 1960, Phys. Rev. 119, 1939.

_- W. L. Fite, D. G. Hummer, and R. T. Brackmann, 1961, Phys. Rev. 124, 2051.

Stewart, D. T., and E. Gabathuler, 1959, Proc. Phys. Soc. (London) 74,473 .

Sural, D. P., and N. C. Sil, 1966, Proc. Phys. Soc. (London) 87, 201.

Taylor, A. J., and P. G. Burke, 1967, Proc. Phys. Soc. (London) $92,336$.

Thieme, O., 1932, Z. Physik 78, 412.

Thomson, J. J., 1912, Phil. Mag. 23, 449.

Tully, J., 1960, M.Sc. dissertation, London. Quoted by M. J. Seaton, in Atomic and Molecular Processes, D. R. Bates, Ed. (Academic Press Inc., New York, 1962), p. 389.

Vainshtein, L. A., 1961, Opt. i Spektroskopiya 11, 301 [English transl.: Opt. Spectry. USSR 11, 163 (1961) ]. 
_-, 1965, Opt. i Spektroskopiya 18, 947 [English transl.: Opt. Spectry. USSR 18, 538 (1965) ].

—_, and G. G. Dolgov, 1959, Opt. i Spektroskopiya 7 , 3. [English transl.: Opt. Spectry. USSR 7, 1 (1959) ].

—, V. Opykhtin, and L. Presnyakov, 1964a, P. N. Lebedev Institute of Physics Report A-53.

, V. Opykhtin, and L. Presnyakov, 1964b, Zh. Eksperim. i

Teor. Fiz. 47, 2306 [English transl.: Soviet Phys.-JETP 20, 1542 (1965) ].

- , L. Presnyakov, and I. Sobelman, 1963, Zh. Eksperim. i

Teor. Fiz. 45, 2015. [English transl.: Soviet Phys.-JETP 18, 1383 (1964)].

Van Regemorter, H., 1960a, Ann. Astrophys. 23, 817.

- 1960b, Monthly Notices Roy. Astron. Soc. 121, 213.

- 1961, Compt. Rend. 252, 2667.

Veldre, V. Ya., A. V. Lyash, and L. L. Rabik, 1965a, in Atomic Collisions III, Akad. Nauk Latv. SSR Inst. Fiz., V. Ya. Veldre, Ed., Riga, p. 85. [Translation TT-66-12939 available through SLA Translation Center, John Crerar Library, Chicago, p. 73) ].

—, A. V. Lyash, and L. L. Rabik, 1965b, Opt. i Spektroskopiya 19, 319 [English transl.: Opt. Spectry. USSR 19, 182 (1965) ].

—, and L. L. Rabik, 1965, Opt. i Spektroskopiya 19, 474. [English transl.: Opt. Spectry. USSR 19, 265 (1965)].

Veselov, M. G., I. M. Antonova, V. F. Bratsev, and I. V. Kirillova, 1961, Opt. i Spektroskopiya 10, 693 [English transl.: Opt. Spectry. USSR 10, 367 (1961)].

Volkova, L. M., 1959, Izv. Akad. Nauk SSSR Ser. Fiz. 23, 968 [English transl.: Bull Acad. Sci. USSR Phys. Ser. 23, 957 (1959)].

—_, 1959, Opt. i Spektroskopiya 6, 273 [English transl.: Opt. Spectry. USSR 6, 179 (1959) ].

— , 1961, Opt. i Spektroskopiya 11, 775 [English transl.: Opt. Spectry USSR 11, 420 (1961) ].

_, 1962, Opt. i Spektroskopiya 13, 849 [English transl.: Opt. Spectry. USSR 13, 482 (1962) ].

[English transl.: Opt. Spectry. USSR 7, 480 (1959)].

, and A. M. Devyatov, 1963, Izv. Akad. Nauk SSSR Ser. Fiz. 27, 1052 [English transl.: Bull. Acad. Sci. USSR Phys. Ser. 27, 1025 (1963) ].
A. M. Devyatov and A. V. Kuralova, 1960, Izv. Akad. Nauk SSSR Ser. Fiz. 24, 950 [English transl.: Bull Acad. Sci. USSR Phys. Ser. 24, 952 (1960)].

Vriens, L., 1965, Physica 31, 1081.

Wheeler, J. A., 1933, Phys. Rev. 43, 258.

Weiss, A. W., 1963, Astrophys. J. 138, 1262.

Wiese, W. L., M. W. Smith, and B. M. Glennon, 1966, "Atomic Transition Probabilities-Hydrogen Through Neon," National Standard Reference Data Series, National Bureau of Standards 4, Vol. I.

Wolf, R., and W. Maurer, 1940, Z. Physik 115, 410.

Wu, T. Y., 1960, Can. J. Phys. 38, 1654.

Yakhontova, V. E., 1959, Vestnik Leningr. Univ. Ser. Fiz. i Khim. 14, 27.

Yamanouchi, T., Y. Inui, and A. Amemiya, 1940, Proc. Phys. Math. Soc. Japan 22, 847.

Yavorskii, B. M., 1947, Zh. Eksperim. i Teor. Fiz. 17, 315.

Zapesochnyi, I. P., 1954, Vestnik Leningr. Univ., No. 11, 67 [Translation available from American Meteorological Society, AD 297209 (1962)].

—_, 1966, Astron. Zh. 43, 954 [English transl.: Soviet Astron.AJ 10, 766 (1967)].

, and P. V. Feltsan, 1963, Izv. Akad. Nauk SSSR Ser. Fiz. 27, 1040 [English transl.: Bull. Acad. Sci. USSR Phys. Ser. 27,1015 (1963)].

- , and P. V. Feltsan, 1965, Ukr. Fiz. Zh. 11, 1197.

_- and P. V. Feltsan, 1966, Opt. i Spektroskopiya 20, 521

[English transl.: Opt. Spectry. USSR 20, 291 (1966)].

, and L. L. Shimon, 1962, Opt. i Spektroskopiya 13, 621

[English transl.: Opt. Spectry. USSR 13, 355 (1962)].

, and L. L. Shimon, 1965, Opt. i Spektroskopiya 19, 480

[English transl.: Opt. Spectry. USSR 19, 268 (1965) ].

, and L. L. Shimon, 1966a, Opt. i Spektroskopiya 20, 753

[English transl.: Opt. Spectry. USSR 20, 421 (1966)].

, and L. L. Shimon, 1966b, Opt. i Spektroskopiya 21, 261

[English transl.: Opt. Spectry. USSR 21, 155 (1966)].

, L. L. Shimon, and A. K. Soshnikov, 1965, Opt. i Spektroskopiya 19, 864 [English transl.: Opt. Spectry. USSR 19, 480 (1965)].

, and O. B. Shpenik, 1966, Zh. Eksperim. i Teor. Fiz. 50, 890 [English transl.: Soviet Phys.-JETP 23, 592 (1966)]. 
TABLE I. Total cross sections for $s \rightarrow s$ excitations of atomic hydrogen, calculated using the first Born approximation (Vainshtein, 1965). Total cross sections in units of $\pi a_{0}^{2}$.

\begin{tabular}{|c|c|c|c|c|c|c|c|c|c|}
\hline 1.16 & $2.16(-1)$ & $3.93(-2)$ & $1.42(-2)$ & $6.79(-3)$ & $3.79(-3)$ & $2.33(-3)$ & $1.54(-3)$ & $1.07(-3)$ & $1.01(1)$ \\
\hline 1.64 & $2.44(-1)$ & $4.31(-2)$ & $1.54(-2)$ & $7.34(-3)$ & $4.08(-3)$ & $2.51(-3)$ & $1.66(-3)$ & $1.15(-3)$ & 1. $28(1)$ \\
\hline 5.00 & $1.07(-1)$ & $1.83(-2)$ & $6.48(-3)$ & $3.07(-3)$ & $1.70(-3)$ & $1.05(-3)$ & $6.89(-4)$ & $4.79(-4)$ & $6.61(0)$ \\
\hline 6.76 & $8.16(-2)$ & $1.38(-2)$ & $4.89(-3)$ & $2.31(-3)$ & 1. $28(-3)$ & $7.89(-4)$ & $5.20(-4)$ & $3.61(-4)$ & $5.09(0)$ \\
\hline 11.24 & $5.04(-2)$ & $8.51(-3)$ & $3.01(-3)$ & $1.42(-3)$ & $7.88(-4)$ & $4.84(-4)$ & $3.19(-4)$ & $2.21(-4)$ & $3.20(0)$ \\
\hline 17.00 & $3.38(-2)$ & $5.69(-3)$ & $2.01(-3)$ & $9.49(-4)$ & $5.27(-4)$ & $3.23(-4)$ & $2.13(-4)$ & $1.48(-4)$ & $2.16(0)$ \\
\hline 24.04 & $2.41(-2)$ & $4.05(-3)$ & $1.43(-3)$ & $6.75(-4)$ & $3.75(-4)$ & $2.29(-4)$ & $1.51(-4)$ & $1.05(-4)$ & $1.54(0)$ \\
\hline
\end{tabular}

Impact

energy $\epsilon$ in

threshold

units

$2 s \rightarrow 4 s$

$2 s \rightarrow 5 s$

$2 s \rightarrow 6 s$

$2 s \rightarrow 7 s$

$2 s \rightarrow 8 s$

$2 s \rightarrow 9 s$

$3 s \rightarrow 4 s$

$3 s \rightarrow 5 s$

$3 s \rightarrow 6 s$

$1.16 \quad 1.64(0)$

$5.82(-1) \quad 2.80(-1)$

$1.58(-1) \quad 9.91(-2)$

$1.64 \quad 2.05(0)$

$7.20(-1)$

$3.45(-1)$

$1.94(-1)$

$1.21(-1)$

$6.65(-2) \quad 7.57(1)$

$8.14(-2) \quad 1.24(2)$

$1.07(1)$

$3.57(0)$

$2.44 \quad 1.73(0)$

$6.00(-1)$

2. $86(-1)$

$1.61(-1)$

$1.00(-1)$

$6.70(-2) \quad 1.20(2)$

$1.80(1)$

$6.07(0)$

$3.56 \quad 1.31(0)$

$4.53(-1)$

2.15(-1)

$1.21(-1)$

$7.51(-2)$

$5.02(-2) \quad 9.82(1)$

$1.68(1)$

$5.59(0)$

5.00

9.88(-1)

$3.39(-1)$

$1.61(-1)$

$9.00(-2)$

$5.60(-2)$

$3.74(-2)$

$7.69(1)$

1.34(1)

$4.39(0)$

6.76

$7.54(-1)$

$2.58(-1)$

$1.22(-1)$

$6.84(-2)$

$4.25(-2)$

$2.82(-2)$

$6.02(1)$

17.00

$3.14(-1)$

$1.07(-1)$

$5.06(-2)$

$4.23(-2)$

2. $63(-2)$

$1.76(-2) \quad 3.85(1)$

$1.03(1)$

$3.35(0)$

24.04

$2.25(-1)$

$7.65(-2)$

$3.60(-2)$

$2.01(-2)$

1.76(-2)

1. $18(-2)$

2.62(1)

$7.93(0)$

$2.57(0)$

4.97(0)

$1.61(0)$

$1.25(-2)$

$8.37(-3)$

1. $88(1)$

$3.37(0)$

$1.08(0)$

Impact

energy $\epsilon$ in

threshold

units

$$
3
$$
3s $4 s \rightarrow 5 s \quad 4 s \rightarrow 6 s$ $4 s \rightarrow 7 s \quad 4 s \rightarrow 8 s$ $4 s \rightarrow 9$ $5 s \rightarrow 6 s$

$\begin{array}{rll}1.16 & 1.67(0) & 9.30(-1) \\ 1.64 & 2.85(0) & 1.60(0) \\ 2.44 & 2.61(0) & 1.45(0) \\ 3.56 & 2.03(0) & 1.13(0) \\ 5.00 & 1.55(0) & 8.57(-1) \\ 6.76 & 1.19(0) & 6.56(-1) \\ 11.24 & 7.40(-1) & 4.09(-1) \\ 17.00 & 4.98(-1) & 2.75(-1) \\ 24.04 & 3.55(-1) & 1.96(-1)\end{array}$

$\begin{array}{ll}5.80(-1) & 2.71(2) \\ 9.98(-1) & 5.99(2) \\ 9.05(-1) & 6.48(2) \\ 7.02(-1) & 5.58(2) \\ 5.31(-1) & 4.50(2) \\ 4.06(-1) & 3.58(2) \\ 2.53(-1) & 2.32(2) \\ 1.70(-1) & 1.60(2) \\ 1.21(-1) & 1.15(2)\end{array}$

$3.28(1)$
$8.07(1)$
$8.48(1)$
$7.05(1)$
$5.53(1)$
$4.33(1)$
$2.76(1)$
$1.87(1)$
$1.34(1)$

$1.03(1)$

$1.03(1)$
$2.61(1)$

$4.57(0)$

2. $1.20(1)$

$2.50(0)$

$6.65(0)$

$6.40(2)$

$2.70(1)$

1.23(1)

$6.74(0)$

$1.95(3)$

$2.21(1)$

$9.99(0)$

$5.46(0)$

$2.37(3)$

$1.72(1)$

1.34(1)

$7.72(0)$

$4.21(0)$

$2.15(3)$

$5.98(0)$

$3.25(0)$

$1.78(3)$

$8.44(0)$

$3.76(0)$

2.04(0)

$1.44(3)$

$5.71(0)$

2.54(0)

$1.38(0)$

$9.54(2)$

$1.34(1)$

$4.09(0)$

$1.82(0)$

$9.86(-1)$

$6.60(2)$

Impact

energy $\epsilon$ in

threshold

units

$5 s \rightarrow 7 s \quad 5 s \rightarrow 8 s \quad 5 s \rightarrow 9 s \quad 6 s \rightarrow 7 s \quad 6 s \rightarrow 8 s \quad 6 s \rightarrow 9 s$

$\begin{array}{rllllll}1.16 & 6.51(1) & 1.85(1) & 7.98(0) & 1.15(3) & 9.06(1) & 2.43(1) \\ 1.64 & 2.47(2) & 7.72(1) & 3.47(1) & 4.82(3) & 5.82(2) & 1.76(2) \\ 2.44 & 2.93(2) & 9.09(1) & 4.05(1) & 6.61(3) & 8.45(2) & 2.47(2) \\ 3.56 & 2.58(2) & 7.80(1) & 3.44(1) & 6.43(3) & 7.92(2) & 2.25(2) \\ 5.00 & 2.08(2) & 6.19(1) & 2.71(1) & 5.59(3) & 6.44(2) & 1.82(2) \\ 6.76 & 1.64(2) & 4.86(1) & 2.12(1) & 4.69(3) & 5.04(2) & 1.43(2) \\ 11.24 & 1.06(2) & 3.11(1) & 1.35(1) & 3.29(3) & 3.10(2) & 9.00(1) \\ 17.00 & 7.22(1) & 2.11(1) & 9.14(0) & 2.39(3) & 2.06(2) & 6.01(1) \\ 24.04 & 5.19(1) & 1.52(1) & 6.56(0) & 1.81(3) & 1.49(2) & 4.25(1)\end{array}$

The numbers in parentheses denote powers of 10. 
TABLE II. Total cross sections for $s \rightarrow p$ excitations of atomic hydrogen, calculated using the first Born approximation (Vainshtein, 1965). Total cross sections in units of $\pi a_{0}^{2} .{ }^{2}$

\begin{tabular}{|c|c|c|c|c|c|c|c|c|c|}
\hline $\begin{array}{l}\text { Impact } \\
\text { energy } \epsilon \text { in } \\
\text { threshold } \\
\text { units }\end{array}$ & $1 s \rightarrow 2 p$ & $1 s \rightarrow 3 p$ & $1 s \rightarrow 4 p$ & $1 s \rightarrow 5 p$ & $1 s \rightarrow 6 p$ & $1 s \rightarrow 7 p$ & $1 s \rightarrow 8 p$ & $1 s \rightarrow 9 p$ & $2 s \rightarrow 3 p$ \\
\hline 1.16 & $7.70(-1)$ & $1.41(-1)$ & $5.08(-2)$ & $2.43(-2)$ & $1.35(-2)$ & $8.35(-3)$ & $5.51(-3)$ & $3.83(-3)$ & $2.75(0)$ \\
\hline 1.64 & $1.23(0)$ & $2.12(-1)$ & $7.57(-2)$ & $3.59(-2)$ & $1.99(-2)$ & $1.23(-2)$ & $8.10(-3)$ & $5.62(-3)$ & $1.10(1)$ \\
\hline 2.44 & $1.32(0)$ & $2.18(-1)$ & $7.68(-2)$ & $3.62(-2)$ & $2.01(-2)$ & $1.23(-2)$ & $8.10(-3)$ & $5.63(-3)$ & $1.70(1)$ \\
\hline 3.56 & $1.22(0)$ & $1.96(-1)$ & $6.83(-2)$ & $3.21(-2)$ & $1.77(-2)$ & $1.09(-2)$ & $7.16(-3)$ & $4.96(-3)$ & $1.91(1)$ \\
\hline 5.00 & $1.07(0)$ & $1.69(-1)$ & $5.83(-2)$ & $2.73(-2)$ & $1.51(-2)$ & $9.23(-3)$ & $6.07(-3)$ & $4.21(-3)$ & $1.88(1)$ \\
\hline 6.76 & $9.18(-1)$ & $1.43(-1)$ & $4.93(-2)$ & $2.31(-2)$ & $1.27(-2)$ & $7.78(-3)$ & $5.12(-3)$ & $3.55(-3)$ & $1.76(1)$ \\
\hline 11.24 & $6.88(-1)$ & $1.05(-1)$ & $3.58(-2)$ & $1.67(-2)$ & $9.21(-3)$ & $5.63(-3)$ & $3.70(-3)$ & $2.56(-3)$ & $1.43(1)$ \\
\hline 17.00 & $5.26(-1)$ & $7.91(-2)$ & $2.70(-2)$ & $1.26(-2)$ & $6.91(-3)$ & $4.22(-3)$ & $2.77(-3)$ & $1.92(-3)$ & $1.15(1)$ \\
\hline 24.04 & $4.15(-1)$ & $6.18(-2)$ & $2.09(-2)$ & $9.76(-3)$ & $5.37(-3)$ & $3.28(-3)$ & $2.15(-3)$ & $1.49(-3)$ & $9.45(0)$ \\
\hline $\begin{array}{l}\text { Impact } \\
\text { energy } \epsilon \text { in } \\
\text { threshold } \\
\text { units }\end{array}$ & $2 s \rightarrow 4 p$ & $2 s \rightarrow 5 p$ & $2 s \rightarrow 6 p$ & $2 s \rightarrow 7 p$ & $2 s \rightarrow 8 p$ & $2 s \rightarrow 9 p$ & $3 s \rightarrow 4 p$ & $3 s \rightarrow 5 p$ & $3 s \rightarrow 6 p$ \\
\hline 1.16 & $6.03(-1)$ & $2.43(-1)$ & $1.23(-1)$ & $7.20(-2)$ & $4.59(-2)$ & $3.12(-2)$ & $3.25(1)$ & $5.29(0)$ & $1.83(0)$ \\
\hline 1.64 & $2.18(0)$ & $8.29(-1)$ & $4.12(-1)$ & $2.38(-1)$ & $1.51(-1)$ & $1.02(-1)$ & $4.88(1)$ & $9.02(0)$ & $3.37(0)$ \\
\hline 2.44 & $3.13(0)$ & $1.16(0)$ & $5.66(-1)$ & $3.24(-1)$ & $2.04(-1)$ & $1.37(-1)$ & $8.56(1)$ & $1.55(1)$ & $5.70(0)$ \\
\hline 3.56 & $3.31(0)$ & $1.19(0)$ & $5.82(-1)$ & $3.31(-1)$ & $2.07(-1)$ & $1.39(-1)$ & $1.10(2)$ & $1.87(1)$ & $6.67(0)$ \\
\hline 5.00 & $3.14(0)$ & $1.12(0)$ & $5.38(-1)$ & $3.04(-1)$ & $1.91(-1)$ & $1.28(-1)$ & $1.18(2)$ & $1.90(1)$ & $6.65(0)$ \\
\hline 6.76 & $2.83(0)$ & $9.99(-1)$ & $4.78(-1)$ & $2.70(-1)$ & $1.69(-1)$ & $1.13(-1)$ & $1.16(2)$ & $1.80(1)$ & $6.20(0)$ \\
\hline 11.24 & $2.23(0)$ & $7.75(-1)$ & $3.69(-1)$ & $2.07(-1)$ & $1.29(-1)$ & $8.65(-2)$ & $1.02(2)$ & $1.45(1)$ & $5.05(0)$ \\
\hline 17.00 & $1.76(0)$ & $6.05(-1)$ & $2.87(-1)$ & $1.61(-1)$ & $1.00(-1)$ & $6.70(-2)$ & $8.55(1)$ & $1.21(1)$ & $4.05(0)$ \\
\hline 24.04 & $1.41(0)$ & $4.83(-1)$ & $2.28(-1)$ & $1.28(-1)$ & $7.96(-2)$ & $5.32(-2)$ & $7.12(1)$ & $9.91(0)$ & $3.21(0)$ \\
\hline $\begin{array}{l}\text { Impact } \\
\text { energy } \epsilon \text { in } \\
\text { threshold } \\
\text { units }\end{array}$ & $3 s \rightarrow 7 p$ & $3 s \rightarrow 8 p$ & $3 s \rightarrow 9 p$ & $4 s \rightarrow 5 p$ & $4 s \rightarrow 6 p$ & $4 s \rightarrow 7 p$ & $4 s \rightarrow 8 p$ & $4 s \rightarrow 9 p$ & $5 s \rightarrow 6 p$ \\
\hline 1.16 & $8.69(-1)$ & $4.90(-1)$ & $3.07(-1)$ & $2.66(2)$ & $3.81(1)$ & $1.25(1)$ & $5.74(0)$ & $3.17(0)$ & $1.15(3)$ \\
\hline 1.64 & $1.67(0)$ & $9.72(-1)$ & $6.21(-1)$ & $2.19(2)$ & $3.41(1)$ & $1.18(1)$ & $5.64(0)$ & $3.20(0)$ & $9.33(2)$ \\
\hline 2.44 & $2.80(0)$ & $1.61(0)$ & $1.03(0)$ & $3.01(2)$ & $5.16(1)$ & $1.85(1)$ & $8.99(1)$ & $5.15(0)$ & $9.08(2)$ \\
\hline 3.56 & $3.23(0)$ & $1.84(0)$ & $1.16(0)$ & $4.05(2)$ & $6.66(1)$ & $2.33(1)$ & $1.12(1)$ & $6.34(0)$ & $1.16(3)$ \\
\hline 5.00 & $3.18(0)$ & $1.80(0)$ & $1.13(0)$ & $4.62(2)$ & $7.18(1)$ & $2.45(1)$ & $1.16(1)$ & $6.52(0)$ & $1.36(3)$ \\
\hline 6.76 & $2.94(0)$ & $1.66(0)$ & $1.04(0)$ & $4.76(2)$ & $7.06(1)$ & $2.37(1)$ & $1.11(1)$ & $6.19(0)$ & $1.45(3)$ \\
\hline 11.24 & $2.37(0)$ & $1.33(0)$ & $8.31(-1)$ & $4.41(2)$ & $6.14(1)$ & $2.00(1)$ & $9.25(0)$ & $5.13(0)$ & $1.41(3)$ \\
\hline 17.00 & $1.89(0)$ & $1.06(0)$ & $6.59(-1)$ & $3.84(2)$ & $5.11(1)$ & $1.64(1)$ & $7.53(0)$ & $4.16(0)$ & $1.25(3)$ \\
\hline 24.04 & $1.51(0)$ & $8.53(-1)$ & $5.31(-1)$ & $3.28(2)$ & $4.25(1)$ & $1.35(1)$ & $6.17(0)$ & $3.39(0)$ & $1.09(3)$ \\
\hline $\begin{array}{l}\text { Impact } \\
\text { energy } \epsilon \text { in } \\
\text { threshold } \\
\text { units }\end{array}$ & $5 s \rightarrow 7 p$ & $5 s \rightarrow 8 p$ & $5 s \rightarrow 9 p$ & $6 s \rightarrow 7 p$ & $6 s \rightarrow 8 p$ & $6 s \rightarrow 9 p$ & $7 s \rightarrow 8 p$ & $7 s \rightarrow 9 p$ & $8 s \rightarrow 9 p$ \\
\hline 1.16 & $1.52(2)$ & $4.82(1)$ & $2.16(1)$ & $3.33(3)$ & $3.93(2)$ & $1.18(2)$ & $4.23(3)$ & $6.32(2)$ & $5.75(3)$ \\
\hline 1.64 & $1.29(2)$ & $4.12(1)$ & $1.88(1)$ & $3.19(3)$ & $4.28(2)$ & $1.29(2)$ & $8.47(3)$ & $1.05(3)$ & $1.53(4)$ \\
\hline 2.44 & $1.42(2)$ & $4.93(1)$ & $2.34(1)$ & $2.43(3)$ & $3.64(2)$ & $1.19(2)$ & $9.16(3)$ & $7.43(2)$ & $2.11(4)$ \\
\hline 3.56 & $1.84(2)$ & $6.32(1)$ & $2.98(1)$ & $2.59(3)$ & $4.89(2)$ & $1.52(2)$ & $8.22(3)$ & $7.04(2)$ & $2.23(4)$ \\
\hline 5.00 & $2.06(2)$ & $6.88(1)$ & $3.21(1)$ & $2.94(3)$ & $6.23(2)$ & $1.77(2)$ & $6.96(3)$ & $8.23(2)$ & $2.12(4)$ \\
\hline 6.76 & $2.09(2)$ & $6.82(1)$ & $3.14(1)$ & $3.13(3)$ & $7.03(2)$ & $1.84(2)$ & $5.84(3)$ & $9.39(2)$ & $1.94(4)$ \\
\hline 11.24 & $1.90(2)$ & $5.95(1)$ & $2.70(1)$ & $3.11(3)$ & $7.28(2)$ & $1.72(2)$ & $4.17(3)$ & $1.03(3)$ & $1.53(4)$ \\
\hline 17.00 & $1.62(2)$ & $4.95(1)$ & $2.24(1)$ & $2.82(3)$ & $6.71(2)$ & $1.50(2)$ & $3.10(3)$ & $9.87(2)$ & $1.23(4)$ \\
\hline 24.04 & $1.37(2)$ & $4.11(1)$ & $1.85(1)$ & $2.48(3)$ & $5.96(2)$ & $1.28(2)$ & $2.38(3)$ & $9.01(2)$ & $9.90(3)$ \\
\hline
\end{tabular}

a The numbers in parentheses denote powers of 10. 
TABLE III. Total cross sections for $s \rightarrow d$ excitations of atomic hydrogen, calculated using the first Born approximation (Vainshtein, 1965). Total cross sections in units of $\pi a_{0}{ }^{2}$.

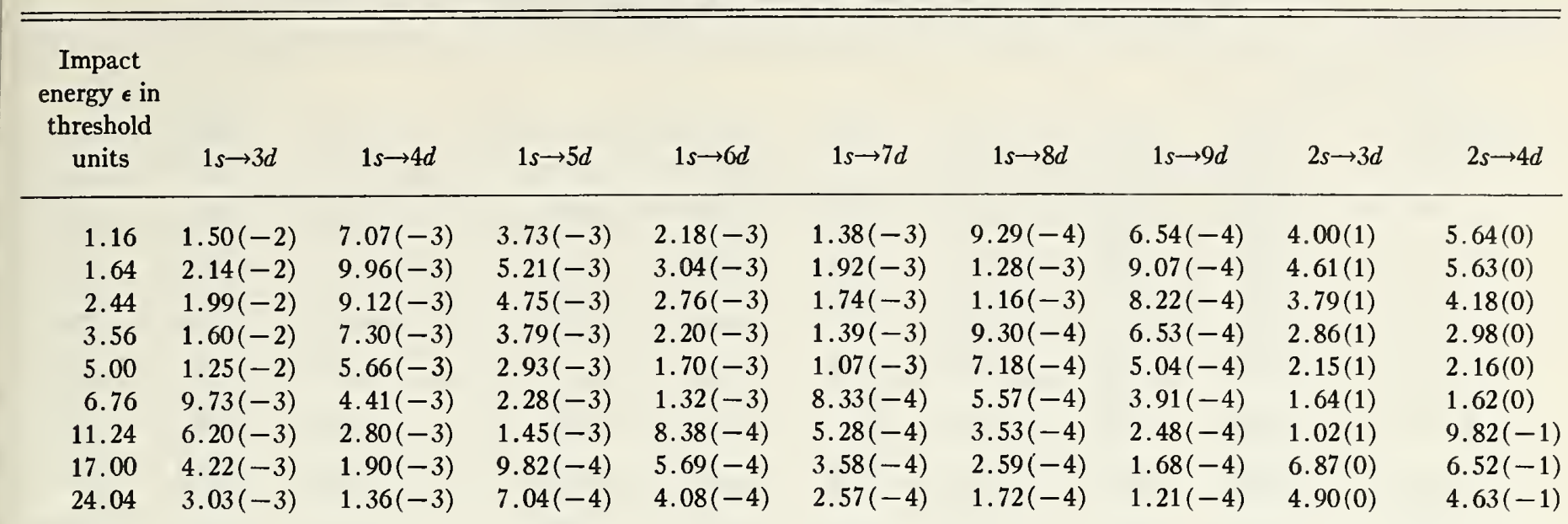

\begin{tabular}{|c|c|c|c|c|c|c|c|c|c|}
\hline $\begin{array}{l}\text { Impact } \\
\text { energy } \epsilon \text { in } \\
\text { threshold } \\
\text { units }\end{array}$ & $2 s \rightarrow 5 d$ & $2 s \rightarrow 6 d$ & $2 s \rightarrow 7 d$ & $2 s \rightarrow 8 d$ & $2 s \rightarrow 9 d$ & $3 s \rightarrow 4 d$ & $3 s \rightarrow 5 d$ & $3 s \rightarrow 6 d$ & $3 s \rightarrow 7 d$ \\
\hline 1.16 & $1.88(0)$ & $8.78(-1)$ & $4.87(-1)$ & $3.01(-1)$ & $2.01(-1)$ & $1.47(2)$ & $2.67(1)$ & $9.81(0)$ & $4.80(0)$ \\
\hline 1.64 & $1.77(0)$ & $8.03(-1)$ & $4.39(-1)$ & $2.69(-1)$ & $1.78(-1)$ & $2.50(2)$ & $3.96(1)$ & $1.36(1)$ & $6.41(0)$ \\
\hline 5.00 & $6.44(-1)$ & $2.84(-1)$ & $1.53(-1)$ & $9.32(-2)$ & $6.13(-2)$ & $1.56(2)$ & $1.95(1)$ & $6.12(0)$ & $2.76(0)$ \\
\hline 6.76 & $4.78(-1)$ & $2.11(-1)$ & $1.14(-1)$ & $6.90(-2)$ & $4.54(-2)$ & $1.22(2)$ & $1.49(1)$ & $4.61(0)$ & $2.07(0)$ \\
\hline 11.24 & $2.89(-1)$ & $1.27(-1)$ & $6.85(-2)$ & $4.16(-2)$ & $2.73(-2)$ & $7.83(1)$ & $9.18(0)$ & $2.82(0)$ & $1.26(0)$ \\
\hline 17.00 & $1.91(-1)$ & $8.42(-2)$ & $4.53(-2)$ & $2.75(-2)$ & $1.81(-2)$ & $5.33(1)$ & $6.14(0)$ & $1.87(0)$ & $8.39(-1)$ \\
\hline 24.04 & $1.35(-1)$ & $5.95(-2)$ & $3.20(-2)$ & $1.94(-2)$ & $1.28(-2)$ & $3.83(1)$ & $4.37(0)$ & $1.33(0)$ & $5.95(-1)$ \\
\hline
\end{tabular}

\section{Impact}

energy $\epsilon$ in

threshold

units

$3 s \rightarrow 8 d$

$3 s \rightarrow 9 d \quad 4 s \rightarrow 5 d$

$4 s \rightarrow 6 d$

$4 s \rightarrow 7 d$

$4 s \rightarrow 8 d$

$4 s \rightarrow 9 d$

$5 s \rightarrow 6 d$

$5 s \rightarrow 7 d$

1.16
1.64
2.44
3.56
5.00
6.76
11.24
17.00
24.04

energy $\epsilon$ in

threshold

units

$2.76(0)$

$1.75(0)$

2.84(2)

5.37 (1)

$2.00(1)$

$9.93(0)$

$5.77(0)$

$4.95(2)$

$7.80(1)$

$3.59(0)$

2. $24(0)$

$7.98(2)$

4.84(1)

2.32 (1)

$1.31(1)$

$1.90(3)$

$3.32(2)$

$1.37(2)$
$1.41(2)$

4. 69(1)

$2.18(1)$

$1.27(0)$

$9.32(2)$
$8.30(2)$

1.14(2)

$3.68(1)$
$2.79(1)$

1. $68(1)$

$2.07(0)$
$1.51(0)$

9. $27(-1)$

$6.79(2)$

$8.85(1)$

$5.46(2)$

$6.85(1)$

2.13(1)

1.26(1)

1.21(1)

2. $66(3)$

$9.20(0)$

2.57(3)

4.13(2)

$1.13(0)$

$4.20(-1) \quad 3.58(2)$

4.31(1)

$1.32(1)$

9.54(0)

(0)

2. 20 (3)

$3.62(2)$

$2.47(2)$

$2.91(1)$

$8.86(0)$

$5.88(0)$

$5.18(0)$

$1.81(3)$

2. $90(2)$

$4.56(-1)$

$2.79(-1)$

$1.79(2)$

$2.08(1)$

$6.31(0)$

$3.93(0)$

$3.19(0)$

$1.22(3)$

$2.29(2)$

$2.12(0)$

$8.51(2)$

$1.46(2)$

$2.80(0)$

$1.51(0)$

$6.21(2)$

$9.97(1)$

7.16(1)

\begin{tabular}{|c|c|c|c|c|c|c|c|c|}
\hline units & $5 s \rightarrow 8 d$ & $5 s \rightarrow 9 d$ & $6 s \rightarrow 7 d$ & $6 s \rightarrow 8 d$ & $6 s \rightarrow 9 d$ & $7 s \rightarrow 8 d$ & $7 s \rightarrow 9 d$ & $8 s \rightarrow 9 d$ \\
\hline 1.16 & $2.71(1)$ & $1.31(1)$ & $1.16(3)$ & $1.36(2)$ & $2.18(0)$ & $1.67(3)$ & $3.10(2)$ & $3.88(3)$ \\
\hline 1.64 & $1.19(2)$ & $5.74(1)$ & $3.61(3)$ & $6.11(2)$ & $2.37(0)$ & $1.39(3)$ & $7.40(2)$ & $5.87(3)$ \\
\hline 2.44 & $1.38(2)$ & $6.42(1)$ & $5.78(3)$ & $1.05(3)$ & $1.77(0)$ & $1.00(3)$ & $1.73(3)$ & $5.46(3)$ \\
\hline 3.56 & $1.16(2)$ & $5.25(1)$ & $5.97(3)$ & $1.10(3)$ & $1.26(0)$ & $7.52(2)$ & $2.13(3)$ & $4.37(3)$ \\
\hline 5.00 & $9.01(1)$ & $4.03(1)$ & $5.28(3)$ & $9.76(2)$ & $9.17(-1)$ & $5.78(2)$ & $2.06(3)$ & $3.40(3)$ \\
\hline 6.76 & $6.97(1)$ & $3.18(1)$ & $4.43(3)$ & $8.19(2)$ & $6.86(-1)$ & $4.51(2)$ & $1.82(3)$ & $2.65(3)$ \\
\hline 11.24 & $4.38(1)$ & $1.93(1)$ & $3.04(3)$ & $5.61(2)$ & $4.18(-1)$ & $2.89(2)$ & $1.32(3)$ & $1.68(3)$ \\
\hline 17.00 & $2.95(1)$ & $1.30(1)$ & $2.15(3)$ & $3.95(2)$ & $2.78(-1)$ & $1.98(2)$ & $9.52(2)$ & $1.13(3)$ \\
\hline 24.04 & $2.10(1)$ & $9.25(0)$ & $1.57(3)$ & $2.89(2)$ & $1.98(-1)$ & $1.43(2)$ & $7.07(2)$ & $8.17(2)$ \\
\hline
\end{tabular}


TABLE IV. Total cross sections for the excitation of discrete levels of atomic hydrogen from the $1 \mathrm{~s}$ state, calculated using the first Born approximation (McCarroll, 1957; Omidvar, 1965). Total cross sections in units of $\pi a_{0}^{2}$.

\begin{tabular}{ccccccccccc}
\hline \hline \multicolumn{2}{c}{ Impact energy } & & & & & & & & & \\
(rydbergs) & $(\mathrm{eV})$ & $Q_{1,2}$ & $Q_{1,3}$ & $Q_{1,4}$ & $Q_{1.5}$ & $Q_{1,6}$ & $Q_{1,7}$ & $Q_{1.8}$ & $Q_{1,9}$ & $Q_{1,10}$ \\
\hline 1.00 & 13.60 & 1.2868 & 0.1787 & 0.0509 & 0.0199 & 0.0092 & 0.0050 & 0.0032 & 0.0018 & 0.0012 \\
1.44 & 19.58 & 1.5354 & 0.2782 & 0.1000 & 0.0476 & 0.0265 & 0.0163 & 0.0104 & 0.0075 & 0.0054 \\
1.96 & 26.66 & 1.4993 & 0.2798 & 0.1021 & 0.0490 & 0.0274 & 0.0169 & 0.0112 & 0.0078 & 0.0056 \\
2.56 & 33.43 & 1.3886 & 0.2600 & 0.0951 & 0.0457 & 0.0256 & 0.0158 & 0.0104 & 0.0073 & 0.0053 \\
3.24 & 44.06 & 1.2630 & 0.2358 & 0.0862 & 0.0413 & 0.0232 & 0.0143 & 0.0096 & 0.0066 & 0.0048 \\
4.00 & 54.40 & 1.1424 & 0.2123 & 0.0775 & 0.0372 & 0.0208 & 0.0128 & 0.0088 & 0.0059 & 0.0043 \\
6.25 & 85.00 & 0.8919 & 0.1637 & 0.0595 & 0.0285 & 0.0160 & 0.0098 & 0.0064 & 0.0045 & 0.0033 \\
9.00 & 122.40 & 0.7101 & 0.1290 & 0.0468 & 0.0224 & 0.0125 & 0.0077 & 0.0048 & 0.0035 & 0.0026 \\
12.25 & 166.60 & 0.5780 & 0.1041 & 0.0377 & 0.0180 & 0.0100 & & & & \\
16.00 & 217.60 & 0.4797 & 0.0858 & 0.0310 & 0.0148 & 0.0083 & & & & \\
20.25 & 275.40 & 0.4050 & 0.0721 & 0.0260 & 0.0124 & 0.0069 & & & & \\
25.00 & 340.00 & 0.3468 & 0.0614 & 0.0221 & 0.0105 & 0.0059 & & & & \\
36.00 & 489.60 & 0.2634 & 0.0463 & 0.0166 & 0.0079 & 0.0044 & & & & \\
49.00 & 666.40 & 0.2075 & 0.0363 & 0.0130 & 0.0062 & 0.0034 & & & & \\
72.25 & 989.40 & 0.1526 & 0.0265 & 0.0095 & 0.0045 & 0.0025 & & & & \\
\hline \hline
\end{tabular}

TABLE V. Total cross sections for the excitation of discrete levels of atomic hydrogen from the $2 s$ state, calculated using the first Born approximation (Boyd, 1959). Total cross sections in units of $\pi a_{0}^{2}$.

\begin{tabular}{ccccccccc}
\hline \hline $\begin{array}{c}\text { Impact energy } \\
\text { (rydbergs) }\end{array}$ & $Q_{2 s, 3}$ & $Q_{2 s, 4}$ & $Q_{28,5}$ & $Q_{2,8}$ & $Q_{2,7}$ & $Q_{2,8}$ & $Q_{2,9}$ & $Q_{2,, 10}$ \\
\hline 0.36 & 65.019 & 12.330 & 4.658 & 2.312 & 1.331 & 0.843 & 0.569 & 0.404 \\
0.64 & 49.441 & 9.454 & 3.612 & 1.808 & 1.047 & 0.664 & 0.450 & 0.320 \\
1.00 & 37.667 & 7.150 & 2.726 & 1.364 & 0.790 & 0.499 & 0.339 & 0.241 \\
1.44 & 29.488 & 5.560 & 2.114 & 1.056 & 0.611 & 0.390 & 0.264 & 0.188 \\
1.96 & 23.703 & 4.446 & 1.687 & 0.842 & 0.487 & 0.309 & 0.209 & 0.149 \\
2.56 & 19.488 & 3.640 & 1.379 & 0.687 & 0.397 & 0.252 & 0.170 & 0.121 \\
3.24 & 16.326 & 3.040 & 1.149 & 0.572 & 0.331 & 0.210 & 0.142 & 0.101 \\
4.00 & 13.895 & 2.580 & 0.974 & 0.485 & 0.280 & 0.179 & 0.121 & 0.086 \\
4.84 & 11.984 & 2.200 & 0.837 & 0.416 & 0.240 & 0.153 & 0.103 & 0.073 \\
5.76 & 10.452 & 1.933 & 0.728 & 0.362 & 0.209 & 0.133 & 0.090 & 0.064 \\
6.76 & 9.206 & 1.699 & 0.640 & 0.318 & 0.183 & 0.116 & 0.079 & 0.056 \\
7.84 & 8.176 & 1.507 & 0.567 & 0.281 & 0.162 & 0.103 & 0.070 & 0.050 \\
9.00 & 7.317 & 1.347 & 0.506 & 0.251 & 0.145 & 0.091 & 0.062 & 0.044 \\
\hline
\end{tabular}



Born approximation (McCrea and McKirgen, 1960). Total cross sections in units of $\pi a_{0}^{2}$.

\begin{tabular}{ccccccccc}
$\begin{array}{c}\text { Impact energy } \\
\text { (rydbergs) }\end{array}$ & $Q_{2 p 0,8}$ & $Q_{2 p 0,4}$ & $Q_{2 p 0.5}$ & $Q_{2 p 0,6}$ & $Q_{2 p 0.7}$ & $Q_{2 p 0.8}$ & $Q_{2 p 0.9}$ & $Q_{2 p 0.10}$ \\
\hline 0.36 & 74.837 & 14.426 & 5.259 & 2.499 & 1.409 & 0.892 & 0.601 & 0.424 \\
0.64 & 64.529 & 12.323 & 4.749 & 2.347 & 1.348 & 0.861 & 0.586 & 0.417 \\
1.00 & 52.482 & 9.826 & 3.790 & 1.859 & 1.072 & 0.684 & 0.461 & 0.328 \\
1.44 & 42.803 & 7.833 & 2.993 & 1.465 & 0.844 & 0.531 & 0.361 & 0.255 \\
1.96 & 35.403 & 6.352 & 2.408 & 1.176 & 0.677 & 0.432 & 0.288 & 0.207 \\
2.56 & 29.727 & 5.246 & 1.974 & 0.963 & 0.553 & 0.348 & 0.234 & 0.165 \\
3.24 & 25.324 & 4.406 & 1.649 & 0.802 & 0.461 & 0.291 & 0.196 & 0.138 \\
4.00 & 21.836 & 3.755 & 1.399 & 0.680 & 0.390 & 0.248 & 0.167 & 0.119 \\
4.84 & 19.038 & 3.241 & 1.202 & 0.583 & 0.334 & 0.211 & 0.141 & 0.100 \\
5.76 & 16.759 & 2.828 & 1.045 & 0.506 & 0.290 & 0.182 & 0.125 & 0.087 \\
6.76 & 14.8765 & 2.491 & 0.918 & 0.444 & 0.254 & 0.160 & 0.108 & 0.077 \\
7.84 & 13.304 & 2.213 & 0.807 & 0.393 & 0.225 & 0.143 & 0.095 & 0.066 \\
9.00 & 11.976 & 1.988 & 0.726 & 0.350 & 0.200 & 0.125 & 0.084 & 0.059
\end{tabular}

TABLE VII. Total cross sections for the excitation of discrete levels of atomic hydrogen from the $2 p \pm 1$ states, calculated using the first Born approximation (McCrea and McKirgen, 1960). Total cross sections in units of $\pi a_{0}^{2}$.

\begin{tabular}{ccccccccc}
$\begin{array}{c}\text { Impact energy } \\
\text { (rydbergs) }\end{array}$ & $Q_{2 p_{ \pm 1.3}}$ & $Q_{2 p_{ \pm 1.4}}$ & $Q_{2 p_{ \pm 1.5}}$ & $Q_{2 p_{ \pm 1,6}}$ & $Q_{2 p_{ \pm 1.7}}$ & $Q_{2 p_{ \pm 1.8}}$ & $Q_{2 p_{ \pm 1.9}}$ & $Q_{2 p_{ \pm 1.10}}$ \\
\hline 0.36 & 76.515 & 13.346 & 4.995 & 2.512 & 1.414 & 0.890 & 0.600 & 0.424 \\
0.64 & 57.44 & 10.692 & 4.052 & 2.040 & 1.166 & 0.740 & 0.505 & 0.358 \\
1.00 & 45.051 & 8.181 & 3.077 & 1.537 & 0.883 & 0.560 & 0.378 & 0.270 \\
1.44 & 35.937 & 6.381 & 2.380 & 1.190 & 0.679 & 0.426 & 0.288 & 0.202 \\
1.96 & 29.278 & 5.102 & 1.891 & 0.935 & 0.537 & 0.342 & 0.229 & 0.160 \\
2.56 & 24.313 & 4.173 & 1.538 & 0.758 & 0.435 & 0.268 & 0.180 & 0.128 \\
3.24 & 20.535 & 3.480 & 1.276 & 0.625 & 0.359 & 0.224 & 0.151 & 0.108 \\
4.00 & 17.587 & 2.949 & 1.077 & 0.528 & 0.302 & 0.190 & 0.129 & 0.091 \\
4.84 & 15.248 & 2.533 & 0.922 & 0.451 & 0.258 & 0.164 & 0.110 & 0.078 \\
5.76 & 13.360 & 2.202 & 0.799 & 0.391 & 0.223 & 0.140 & 0.094 & 0.067 \\
6.76 & 11.8125 & 1.934 & 0.700 & 0.341 & 0.195 & 0.123 & 0.082 & 0.058 \\
7.84 & 10.527 & 1.713 & 0.618 & 0.301 & 0.172 & 0.108 & 0.072 & 0.052 \\
9.00 & $9.447_{5}$ & 1.534 & 0.551 & 0.272 & 0.153 & 0.096 & 0.064 & 0.046
\end{tabular}


TABLE VIII. Total cross sections for the excitation of discrete levels of atomic hydrogen from the $n=3$ level, calculated using the first Born approximation (Omidvar, 1965). Total cross sections in units of $\pi a_{0}^{2}$.

\begin{tabular}{|c|c|c|c|c|c|c|c|c|c|c|c|}
\hline $\begin{array}{c}\text { Impact } \\
\text { energy } \\
\text { (rydbergs) }\end{array}$ & $Q_{3.4}$ & $Q_{3,5}$ & $Q_{3,6}$ & $Q_{3,7}$ & $Q_{3,8}$ & $\begin{array}{c}\text { Impact } \\
\text { energy } \\
\text { (rydbergs) }\end{array}$ & $Q_{3,4}$ & $Q_{3,5}$ & $Q_{3,6}$ & $Q_{3,7}$ & $Q_{3.8}$ \\
\hline 0.07 & 657.1 & & & & & 1.44 & 182.5 & 30.34 & 11.19 & 5.55 & 3.23 \\
\hline 0.08 & 709.2 & 83.37 & & & & 1.96 & 145.1 & 23.69 & 8.69 & 4.29 & 2.49 \\
\hline 0.111 & 735.3 & 126.98 & 42.92 & 19.16 & 9.94 & 2.56 & 118.4 & 19.07 & 6.94 & 3.45 & 1.99 \\
\hline 0.64 & 322.3 & 56.35 & 21.16 & 10.62 & 6.21 & 6.25 & 58.5 & 9.12 & 3.25 & 1.61 & 0.97 \\
\hline 1.00 & 237.4 & 40.33 & 14.98 & 7.47 & 4.35 & 9.00 & 43.6 & 6.71 & 2.38 & 1.19 & 0.66 \\
\hline
\end{tabular}

TABLE IX. Values of the constants $C_{n^{\prime} l^{\prime}, n l}$ occurring in the Bethe total cross section formula (47) for $n^{\prime} l^{\prime} \rightarrow n l$ transitions of atomic hydrogen satisfying $n-n^{\prime}=1$ or 2 and $l-l^{\prime}=1^{\text {a }}$ (McCoyd and Milford, 1963).

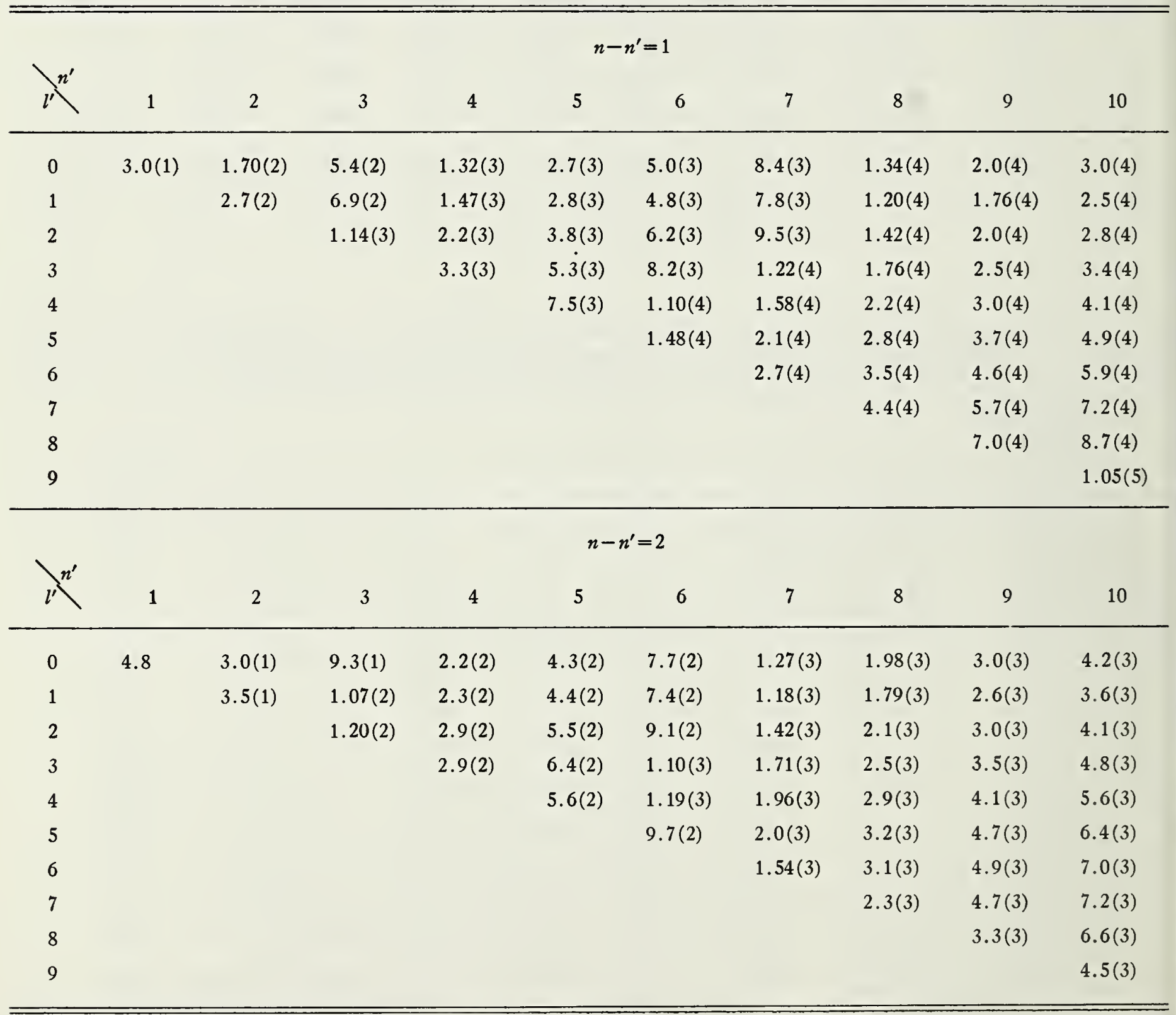

The numbers in parentheses denote powers of 10 . 
TABLE X. Values of the constants $D_{n^{\prime} l^{\prime}, n l}$ occurring in the Bethe total cross section formula (47) for $n^{\prime} l^{\prime} \rightarrow n l$ transitions of atomic hydrogen satisfying $n-n^{\prime}=1$ or 2 and $l-l^{\prime}=1$ (McCoyd and Milford, 1963).

\begin{tabular}{|c|c|c|c|c|c|c|c|c|c|c|}
\hline & & & & & $\imath-n^{\prime}=$ & & & & & \\
\hline$l^{\prime}$ & 1 & 2 & 3 & 4 & 5 & 6 & 7 & 8 & 9 & 10 \\
\hline 0 & $1.03(-1)$ & $2.7(-1)$ & $5.0(-1)$ & $8.0(-1)$ & 1.14 & 1.6 & 2 & 3 & 3 & 4 \\
\hline 1 & & $5.8(-1)$ & $7.6(-1)$ & 1.08 & 1.44 & 1.9 & 3 & 3 & 3 & 5 \\
\hline 2 & & & 1.39 & 1.50 & 1.77 & 2 & 3 & 3 & 4 & 5 \\
\hline 3 & & & & 2.6 & 2.6 & 2 & 4 & 4 & 4 & 5 \\
\hline 4 & & & & & 4.2 & 5 & 4 & 5 & 5 & 6 \\
\hline 5 & & & & & & 5 & 5 & 5 & 5 & 6 \\
\hline 6 & & & & & & & $1.2(1)$ & 8 & 6 & 8 \\
\hline 7 & & & & & & & & $1.1(1)$ & 9 & 8 \\
\hline 8 & & & & & & & & & $1.6(1)$ & 1.2(1) \\
\hline 9 & & & & & & & & & & $1.8(1)$ \\
\hline
\end{tabular}

\begin{tabular}{|c|c|c|c|c|c|c|c|c|c|c|}
\hline \multirow{2}{*}{$l^{n^{\prime}}$} & \multicolumn{10}{|c|}{$n-n^{\prime}=2$} \\
\hline & 1 & 2 & 3 & 4 & 5 & 6 & 7 & 8 & 9 & 10 \\
\hline 0 & $1.41(-1)$ & $3(-1)$ & $5.2(-1)$ & $8.1(-1)$ & 1.2 & 1.8 & 1.8 & 3 & 3 & 4 \\
\hline 1 & & $8(-1)$ & $9.7(-1)$ & 1.2 & 2 & 1.8 & 1.8 & 3 & 4 & 4 \\
\hline 2 & & & 2.7 & 2 & 3 & 3 & 4 & 4 & 5 & 5 \\
\hline 3 & & & & 5.9 & 4 & 5 & 4 & 4 & 5 & 6 \\
\hline 4 & & & & & $1.1(1)$ & 8 & 7 & 8 & 7 & 6 \\
\hline 5 & & & & & & $2(1)$ & $1.3(1)$ & 8 & 7 & 9 \\
\hline 6 & & & & & & & $3(1)$ & $1.5(1)$ & $1.6(1)$ & $1.3(1)$ \\
\hline 7 & & & & & & & & $5(1)$ & $3(1)$ & $1.3(1)$ \\
\hline 8 & & & & & & & & & $7(1)$ & $3(1)$ \\
\hline 9 & & & & & & & & & & $9(1)$ \\
\hline
\end{tabular}

a The numbers in parentheses denote powers of 10.

TABLE XI. Values of the constants $C_{n^{\prime}, n}$ and $D_{n^{\prime}, n}$ occurring in the Bethe total cross section formula (48) for $n^{\prime} \rightarrow n$ transitions of atomic hydrogen satisfying $n-n^{\prime}=1$ or 2 (Kingston and Lauer, $1966 \mathrm{a} ; 1966 \mathrm{~b})$. $^{\mathrm{a}}$

\begin{tabular}{cccccc}
\hline & \multicolumn{2}{c}{$n-n^{\prime}=1$} & & \multicolumn{2}{c}{$n-n^{\prime}=2$} \\
\cline { 2 - 3 } \cline { 5 - 6 } & $C_{n^{\prime}, n}$ & $D_{n^{\prime}, n}$ & & $C_{n^{\prime}, n}$ & $D_{n^{\prime}, n}$ \\
\hline 1 & $3.02(1)$ & $1.46(-1)$ & & $4.84(0)$ & $2.24(-1)$ \\
2 & $2.51(2)$ & $8.61(-1)$ & & $3.46(1)$ & $2.35(0)$ \\
3 & $9.43(2)$ & $2.27(0)$ & & $1.15(2)$ & $9.48(0)$ \\
4 & $2.51(3)$ & $4.36(0)$ & & $2.81(2)$ & $2.42(1)$ \\
5 & $5.48(3)$ & $7.08(0)$ & & $5.75(2)$ & $4.79(1)$ \\
6 & $1.05(4)$ & $1.04(1)$ & & $1.05(3)$ & $8.13(1)$ \\
\hline
\end{tabular}

The numbers in parentheses denote powers of 10 .
TABLE XII. Total cross sections for the $1 s \rightarrow 2 s$ excitation of atomic hydrogen calculated using the first Born approximation, the Born-Oppenheimer approximation and the first-order exchange approximation (Bell and Moiseiwitsch, 1963). Total cross sections in units of $\pi a_{0}^{2} .^{\mathrm{a}}$

\begin{tabular}{cccc}
\hline \hline $\begin{array}{c}\text { Impact energy } \\
\text { (rydbergs) }\end{array}$ & $\mathrm{B}$ & B.O. & $\mathrm{E}_{1}$ \\
\hline 1.00 & 0.248 & 1.648 & 0.447 \\
2.25 & 0.167 & 0.204 & 0.148 \\
4.00 & 0.102 & 0.0958 & 0.0905 \\
9.00 & 0.0477 & 0.0451 & 0.0447 \\
16.00 & 0.0273 & 0.0264 & 0.0263 \\
\hline \hline
\end{tabular}

a $B=$ first Born approximation; B.O.= Born-Oppenheimer approximation; $E_{1}=$ first-order exchange approximation. 
TABLE XIII. Total cross sections for the $1 s \rightarrow 2 p 0$ and $1 s \rightarrow 2 p \pm 1$ excitations of atomic hydrogen calculated using the first Born approximation, the Born-Oppenheimer approximation and the first-order exchange approximation. The axis of quantization of the atomic wave functions is chosen along the direction of the incident electron. Total cross sections in units of $\pi a_{0}^{2}$.

\begin{tabular}{|c|c|c|c|c|c|c|c|c|}
\hline \multirow{2}{*}{$\begin{array}{l}\text { Impact energy } \\
\text { (rydbergs) }\end{array}$} & \multicolumn{3}{|c|}{$Q_{2 p 0^{n}}$} & \multicolumn{2}{|c|}{$Q_{2 p \pm 1}^{b}$} & \multicolumn{3}{|c|}{$Q_{2 p}=Q_{2 p 0}+2 Q_{2 p \pm 1}$} \\
\hline & $\mathrm{B}^{\mathrm{c}}$ & B.O.' & $E_{1} \mathrm{c}$ & B & $\mathrm{BO} \equiv E_{1}$ & B & B.O. & $E_{1}$ \\
\hline 1.00 & 0.873 & 1.498 & 1.209 & 0.0830 & 0.0811 & 1.039 & 1.660 & 1.371 \\
\hline 4.00 & 0.475 & 0.442 & 0.441 & 0.283 & 0.265 & 1.041 & 0.973 & 0.972 \\
\hline 9.00 & 0.231 & 0.227 & 0.227 & 0.215 & 0.211 & 0.662 & 0.648 & 0.648 \\
\hline 16.00 & 0.134 & 0.133 & 0.133 & 0.159 & 0.158 & 0.453 & 0.449 & 0.449 \\
\hline
\end{tabular}

a Bell and Moiseiwitsch, 1963

b Bell, 1965 .

${ }^{c} B=$ first Born approximation; B.O. = Born-Oppenheimer approximation; $E_{1}=$ first-order exchange approximation.

TABLE XIV. Zero-order partial cross sections for the $1 s \rightarrow 2 s$ excitation calculated using the Born-Oppenheimer approximation, the distorted waves approximation and the $1 s \rightarrow 2 s$ close coupling approximation."

Zero-order partial cross sections in units of $\pi a_{0}^{2}$

\begin{tabular}{|c|c|c|c|c|c|c|c|}
\hline \multirow{2}{*}{$\begin{array}{l}\text { Impact } \\
\text { energy } \\
\text { (rydbergs) }\end{array}$} & \multicolumn{3}{|c|}{$\begin{array}{c}\text { Exchange neglected } \\
Q\end{array}$} & \multicolumn{4}{|c|}{$\begin{array}{l}\text { Exchange included, singlet scattering } \\
\qquad Q^{+}\end{array}$} \\
\hline & $\mathbf{B}^{\mathbf{b}}$ & D.W.b & Ce & B.O. ${ }^{b}$ & D.W.b & D.W.d & $\mathrm{Ce}$ \\
\hline 1.00 & 0.198 & 0.239 & 0.204 & 0.287 & 0.714 & 0.0738 & 0.288 \\
\hline 2.25 & 0.0585 & 0.0455 & 0.0450 & 0.014 & 0.127 & 0.069 & 0.0968 \\
\hline 4.00 & 0.0194 & 0.0141 & 0.0155 & 0.018 & 0.0255 & 0.0212 & 0.0290 \\
\hline
\end{tabular}

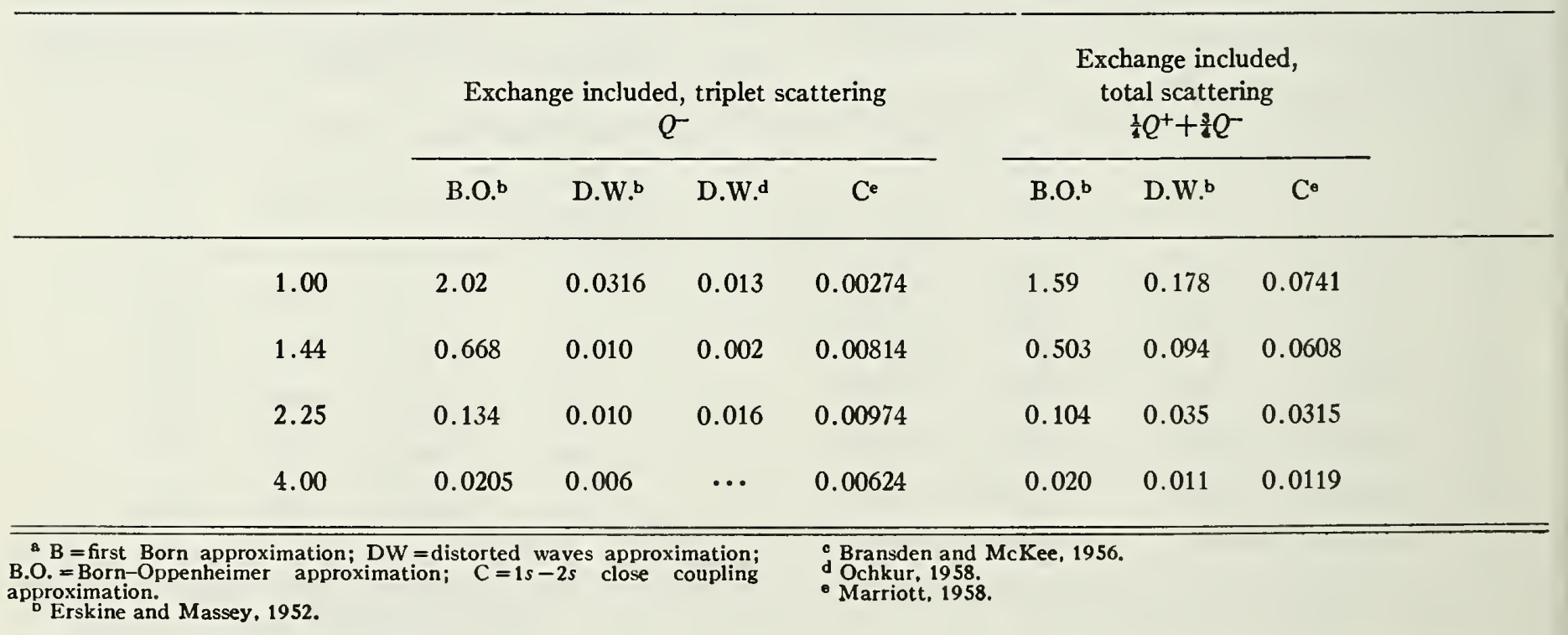


TABLE XV. Total cross sections for various excitations of atomic hydrogen calculated using the distorted waves method neglecting exchange (Vainshtein, 1961). Total cross sections in units of $\pi a_{0}^{2} .^{\mathrm{s}}$

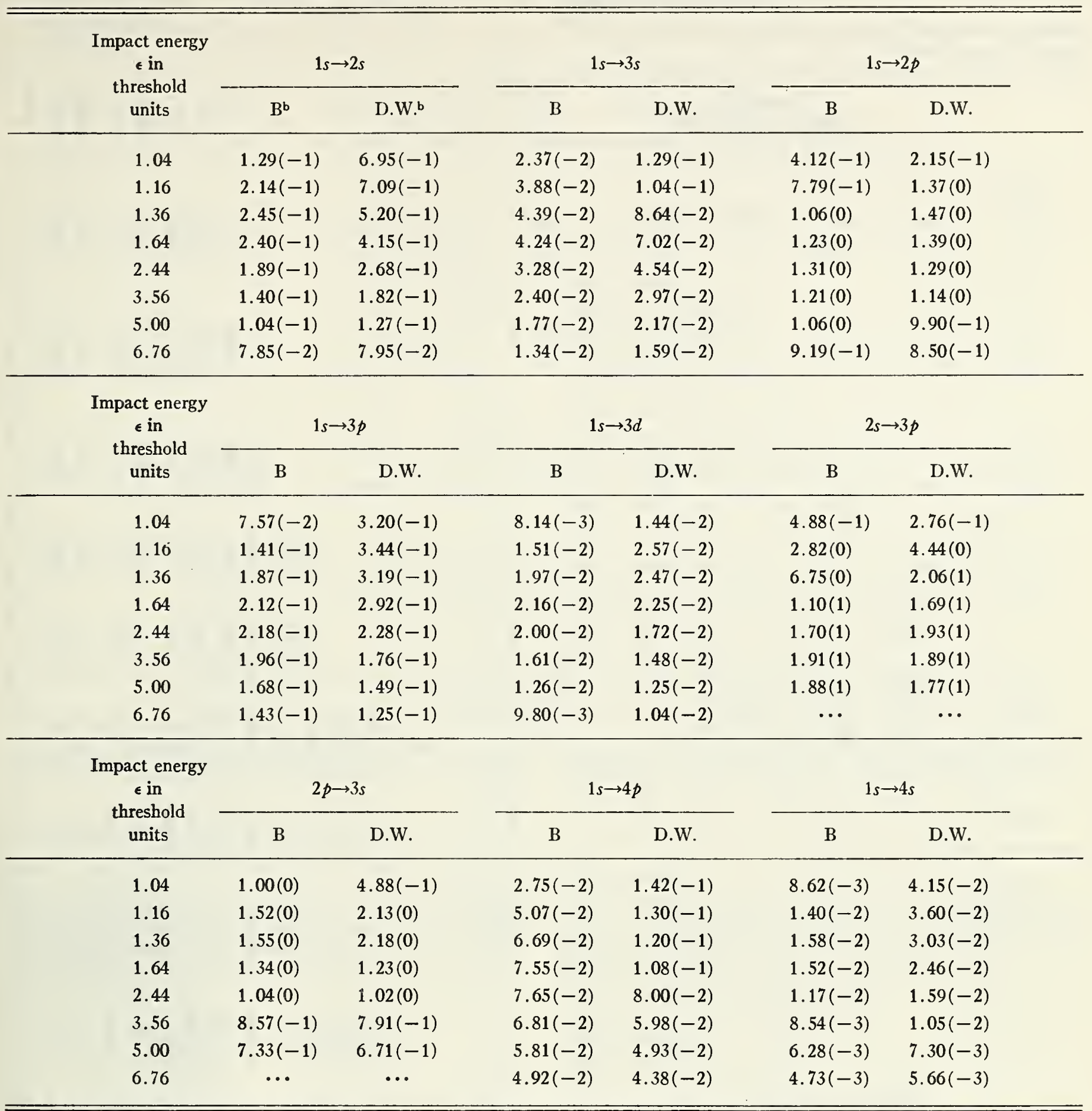

\footnotetext{
The numbers in parentheses denote powers of 10 .

b $\mathrm{B}=$ first Born approximation; D.W. = distorted waves approximation.
}

TABLE XVI. Rate coefficients for $2 s \rightarrow 2 p$ transitions in atomic hydrogen (Seaton, 1955a). Rate coefficients $W$ in $\mathrm{cm}^{3} \mathrm{sec}^{-1}$. $^{\mathrm{s}}$

\begin{tabular}{|c|c|c|c|c|c|c|c|c|}
\hline & \multicolumn{4}{|c|}{$T=1 \times 10^{4} \operatorname{deg} \mathrm{K}$} & \multicolumn{4}{|c|}{$T=2 \times 10^{4} \operatorname{deg} \mathrm{K}$} \\
\hline & I & II & III & IV & $\mathrm{I}$ & II & III & VI \\
\hline $10^{\prime} \times W^{\prime}$ & 0.27 & 0.25 & 0.24 & 0.22 & 0.20 & 0.18 & 0.17 & 0.17 \\
\hline $10^{4} \times W^{\prime \prime}$ & 0.45 & 0.42 & 0.38 & 0.35 & 0.34 & 0.31 & 0.29 & 0.27 \\
\hline
\end{tabular}

${ }^{a} W^{\prime}$ for $2 s \rightarrow 2 p_{1 / 2}$ and $W^{\prime \prime}$ for $2 s \rightarrow 2 p_{3 / 2 .}$ Approximations: I Bethe; II first Born; III semiclassical (Purcell, 1952); IV modified Bethe. 
TABLE XVII. Values of $X_{n+1, n}$ occurring in the formula (96) for the excitation cross section for the $n \rightarrow n+1$ transition of atomic hydrogen calculated using the semiclassical impact parameter method (Saraph, 1964).

\begin{tabular}{|c|c|c|c|c|c|c|}
\hline \multirow[b]{2}{*}{$\log E{ }^{n}$} & \multicolumn{6}{|c|}{$\begin{array}{c}\text { Values of } X_{n+1, n} \\
\text { (Impact energy } E \text { in } \mathrm{eV} \text { ) }\end{array}$} \\
\hline & 1 & 2 & 3 & 4 & 6 & 8 \\
\hline-0.8 & & & & & 1.12 & 1.47 \\
\hline-0.6 & & & & & 1.34 & 1.67 \\
\hline-0.4 & & & & 0.850 & 1.55 & 1.82 \\
\hline-0.2 & & & & 1.28 & 1.70 & 1.93 \\
\hline 0.0 & & & 1.11 & 1.48 & 1.80 & 1.98 \\
\hline 0.2 & & & 1.36 & 1.60 & 1.84 & 1.97 \\
\hline 0.4 & & 0.956 & 1.49 & 1.65 & 1.81 & 1.91 \\
\hline 0.6 & & 1.22 & 1.54 & 1.62 & 1.73 & 1.79 \\
\hline 0.8 & & 1.28 & 1.43 & 1.48 & 1.56 & 1.59 \\
\hline 1.0 & & 1.23 & 1.28 & 1.29 & 1.32 & 1.35 \\
\hline 1.2 & 0.529 & 1.12 & 1.12 & 1.12 & 1.12 & 1.13 \\
\hline 1.4 & 0.640 & 0.985 & 0.981 & 0.950 & 0.944 & 0.948 \\
\hline 1.6 & 0.650 & 0.852 & 0.820 & 0.802 & 0.991 & 0.789 \\
\hline 1.8 & 0.611 & 0.727 & 0.691 & 0.672 & 0.655 & 0.653 \\
\hline 2.0 & 0.555 & 0.616 & 0.580 & 0.561 & & \\
\hline 2.2 & 0.483 & 0.516 & 0.483 & 0.465 & & \\
\hline $\log \sum^{n}$ & 10 & 15 & 20 & 25 & 30 & 40 \\
\hline-2.6 & & & & & 0.99 & 1.31 \\
\hline-2.4 & & & & 1.01 & 1.21 & 1.54 \\
\hline-2.2 & & & & 1.23 & 1.44 & 1.79 \\
\hline-2.0 & & & 1.21 & 1.46 & 1.68 & 2.05 \\
\hline-1.8 & & 1.08 & 1.44 & 1.70 & 1.92 & 2.32 \\
\hline-1.6 & & 1.33 & 1.66 & 1.93 & 2.16 & 2.59 \\
\hline-1.4 & 1.03 & 1.55 & 1.88 & 2.16 & 2.41 & 2.86 \\
\hline-1.2 & 1.29 & 1.77 & 2.10 & 2.38 & 2.64 & 3.11 \\
\hline-1.0 & 1.52 & 1.96 & 2.29 & 2.57 & 2.84 & 3.33 \\
\hline-0.8 & 1.72 & 2.13 & 2.47 & 2.74 & 3.02 & 3.45 \\
\hline-0.6 & 1.88 & 2.28 & 2.60 & 2.88 & 3.14 & 3.58 \\
\hline-0.4 & 2.01 & 2.37 & 2.68 & 2.94 & 3.17 & 3.57 \\
\hline-0.2 & 2.09 & 2.42 & 2.69 & 2.92 & 3.13 & 3.46 \\
\hline 0.0 & 2.12 & 2.40 & 2.64 & 2.81 & 2.99 & 3.30 \\
\hline 0.2 & 2.09 & 2.31 & 2.50 & 2.69 & 2.82 & 2.99 \\
\hline 0.4 & 1.99 & 2.20 & 2.34 & 2.44 & 2.52 & 2.89 \\
\hline 0.6 & 1.89 & 2.00 & 2.08 & 2.15 & 2.20 & 2.29 \\
\hline
\end{tabular}
.

売

.

ษัํํ

งิ

ปิ

혼

ㅇ.

苛.

응.

马्य

莺

รี

은

롱

घี

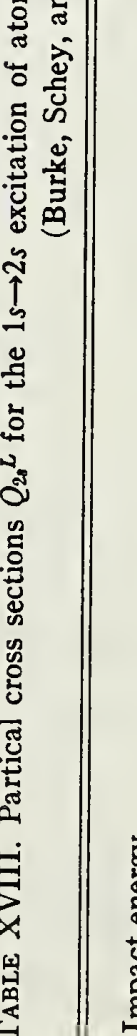

हี

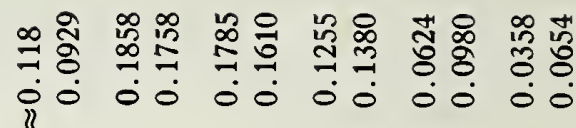

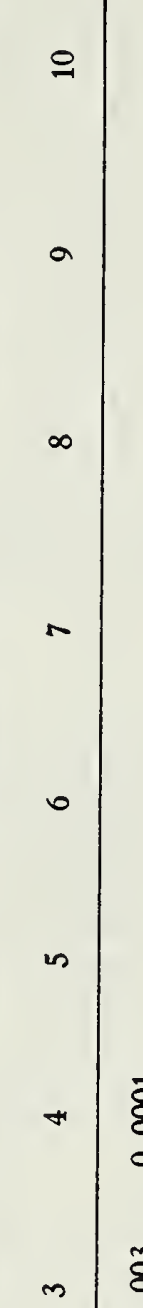

$n$

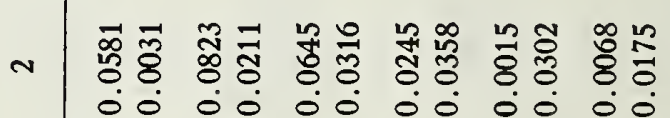

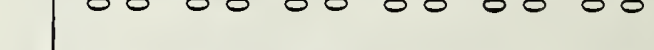

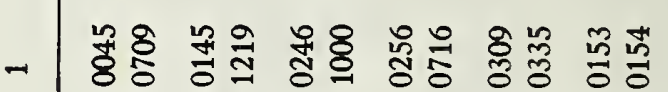

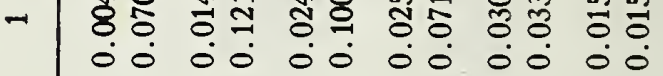

ิำ유ำ

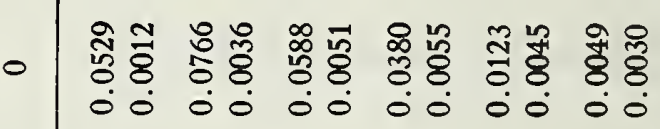
व

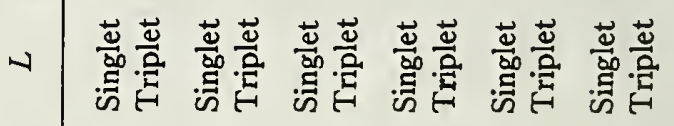

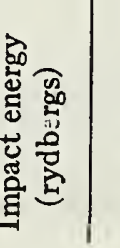

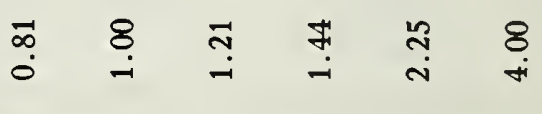


TABLE XIX. Partial cross sections $Q_{2 p}{ }^{L}$ for the $1 s \rightarrow 2 p$ excitation of atomic hydrogen calculated according to the $1 s-2 s-2 p$

\begin{tabular}{|c|c|c|c|c|c|c|c|c|c|}
\hline $\begin{array}{l}\text { Impact energy } \\
\text { (rydbergs) }\end{array}$ & $L$ & 0 & 1 & 2 & 3 & 4 & 5 & 6 & 7 \\
\hline \multirow[t]{2}{*}{0.81} & Singlet & 0.0384 & 0.0754 & 0.1095 & $\approx 0.010$ & 0.0030 & 0 & & \\
\hline & Triplet & 0.0007 & 0.0657 & 0.0083 & 0.0505 & 0.0013 & 0.0001 & & \\
\hline \multirow[t]{2}{*}{1.00} & Singlet & 0.0360 & 0.1105 & 0.2532 & 0.0352 & 0.0098 & 0.0025 & 0.0007 & 0.0002 \\
\hline & Triplet & 0.0033 & 0.0798 & 0.0458 & 0.1671 & 0.0438 & 0.0093 & 0.0020 & 0.0005 \\
\hline \multirow[t]{2}{*}{1.21} & Singlet & 0.0359 & 0.1105 & 0.3404 & 0.0863 & 0.0301 & 0.0112 & 0.0044 & 0.0016 \\
\hline & Triplet & 0.0068 & 0.0629 & 0.0549 & 0.1831 & 0.1046 & 0.0388 & 0.0133 & 0.0050 \\
\hline \multirow[t]{2}{*}{1.44} & Singlet & 0.0343 & 0.0815 & 0.02895 & 0.1256 & 0.0508 & 0.0229 & 0.0108 & $(0.0049)$ \\
\hline & Triplet & 0.0095 & 0.0416 & 0.0539 & 0.1740 & 0.1404 & 0.0732 & 0.0347 & $(0.0147)$ \\
\hline \multirow[t]{2}{*}{2.25} & Singlet & 0.0171 & 0.0176 & 0.0942 & 0.0999 & 0.0695 & 0.0451 & 0.0292 & $(0.0181)$ \\
\hline & Triplet & 0.0106 & 0.0133 & .0 .0357 & 0.1077 & 0.1342 & 0.1148 & 0.0838 & $(0.0542)$ \\
\hline \multirow[t]{2}{*}{4.00} & Singlet & 0.0035 & 0.0024 & 0.0169 & 0.0302 & 0.0347 & 0.0329 & 0.0286 & 0.0237 \\
\hline & Triplet & 0.0052 & 0.0038 & 0.0139 & 0.0394 & 0.0624 & 0.0728 & 0.0719 & 0.0647 \\
\hline
\end{tabular}

TABLE XX. Total cross sections for the $1 s \rightarrow 2 s$ excitation of atomic hydrogen calculated according to the $1 s-2 s-2 p$ close coupling approximation with exchange included (Burke, Schey, and Smith, 1963).

\begin{tabular}{cc}
\hline \hline $\begin{array}{c}\text { Impact } \\
\text { energy } \\
\text { (rydbergs) }\end{array}$ & $\begin{array}{c}\text { Total cross } \\
\text { section in units } \\
\text { of } \pi a_{0}^{2}\end{array}$ \\
\hline 0.81 & 0.211 \\
1.00 & 0.3616 \\
1.21 & 0.3395 \\
1.44 & 0.2635 \\
2.25 & 0.1604 \\
4.00 & 0.1012 \\
\hline \hline
\end{tabular}

TABLE XXI. Total cross sections for the $1 s \rightarrow 2 p$ excitation of atomic hydrogen calculated according to the $1 s-2 s-2 p$ close coupling approximation with exchange included (Burke, Schey, and Smith, 1963). Total cross sections in units of $\pi a_{0}^{2}$.

\begin{tabular}{cccc}
\hline $\begin{array}{c}\text { Impact energy } \\
\text { (rydbergs) }\end{array}$ & $Q_{2 p_{ \pm 1}}$ & $Q_{2 p 0}$ & $Q_{2 p}=\sum_{m=0, \pm 1} Q_{2 p m}$ \\
\hline 0.81 & $\approx 0.068$ & $\approx 0.223$ & $\approx 0.360$ \\
1.00 & 0.0997 & 0.6005 & 0.7999 \\
1.21 & 0.1395 & 0.8149 & 1.0939 \\
1.44 & 0.1631 & 0.8539 & 1.1801 \\
2.25 & 0.2244 & 0.6423 & 1.0911 \\
4.00 & 0.2394 & 0.3928 & 0.8716 \\
\hline
\end{tabular}




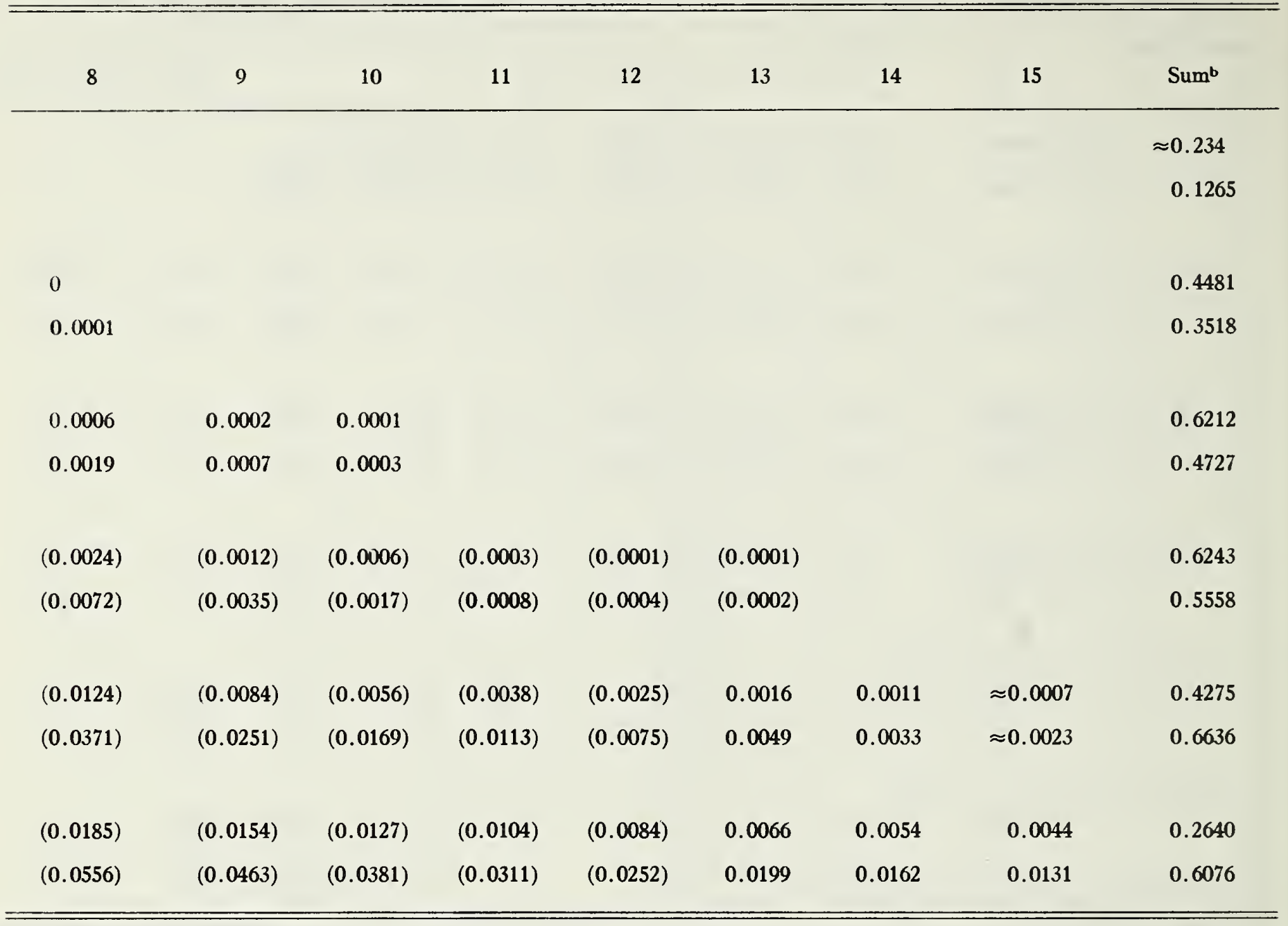

Spin weighting factors included.

TABLE XXII. Cross sections for the $1 s \rightarrow 2 s, 1 s \rightarrow 2 p$, and $1 s \rightarrow 3 p$ excitations of atomic hydrogen calculated according to (a) the $1 s-2 s-2 p-3 s-3 p$, (b) the $1 s-2 s-2 p$, and (c) the $1 s-3 p$ close coupling approximations with exchange included at $16.5-\mathrm{eV}$ electron impact energy (Burke, 1963). Cross sections in units of $\pi a_{0}^{2}$.

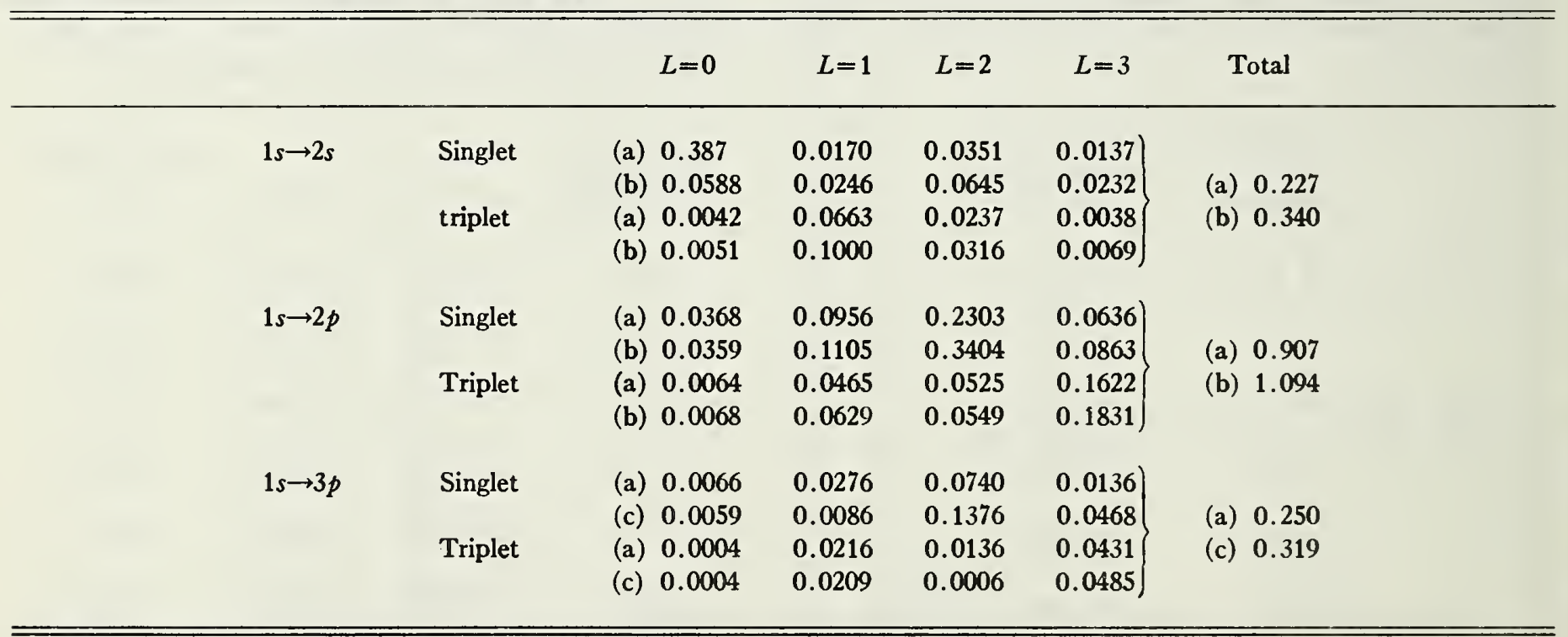

Spin weighting factors included. 
TABLE XXIII. Partial cross sections for the $1 s \rightarrow 2 s$ and $1 s \rightarrow 2 p$ excitations of atomic hydrogen calculated near the threshold energy according to the $1 s-2 s-2 p$ close coupling approximation with exchange included (Damburg and Gailitis, 1963). Partial cross sections in units of $\pi a_{0}^{2}$.

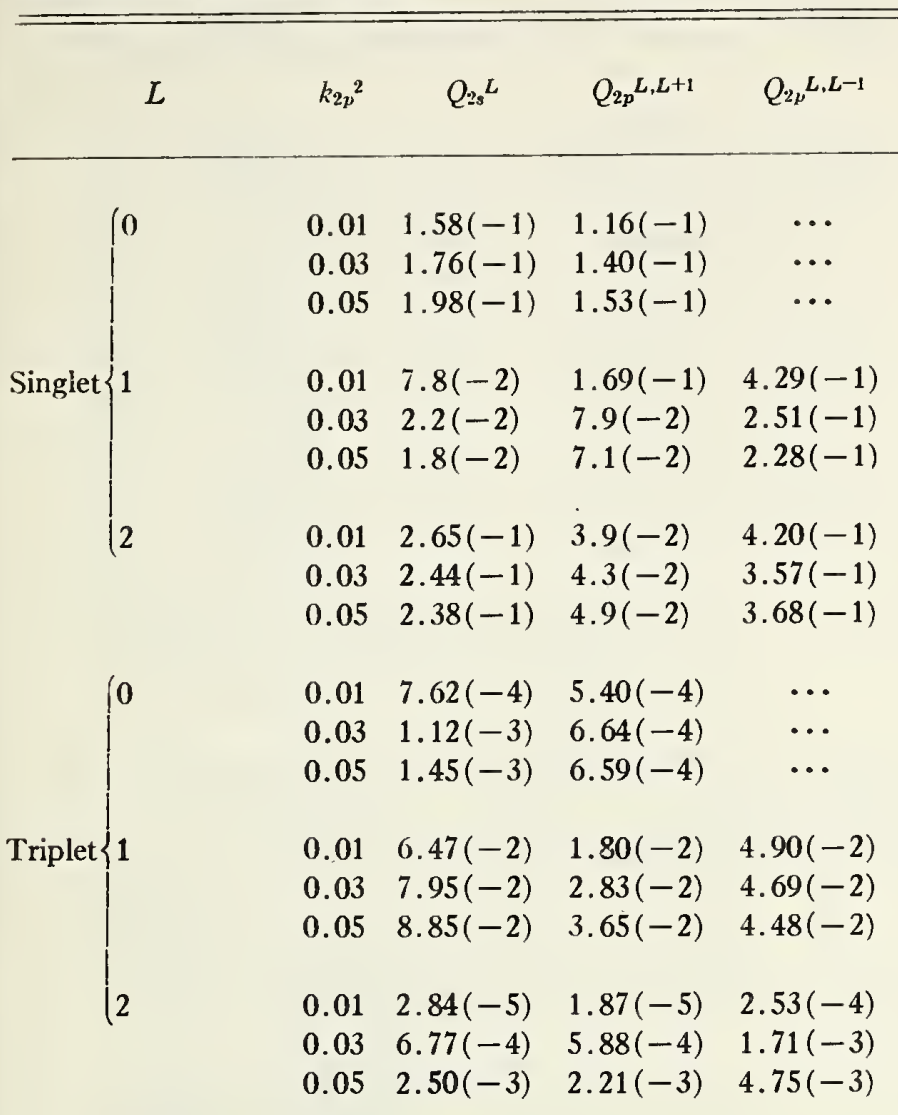

TABLE XXIV. Total cross sections for the $1 s \rightarrow 2 s$ and $1 s \rightarrow 2 p$ excitations of atomic hydrogen calculated near the threshold energy according to the $1 s-2 s-2 p$ close coupling approximation with exchange included (Damburg and Gailitis, 1963). Total cross sections in units of $\pi a_{0}^{2}$.

$k_{2 p}^{2} \quad 1 s \rightarrow 2 s \quad 1 s \rightarrow 2 p$

$\begin{array}{lll}0.10 & 0.174 & 0.344 \\ 0.03 & 0.174 & 0.283 \\ 0.05 & 0.188 & 0.305\end{array}$

TABLE XXV. Total cross sections for the $2^{3} S \rightarrow 2^{3} P, 3^{3} P, 3^{3} D$, and $4^{3} D$ excitations of helium calculated using the first Born approximation and the modified Bethe approximation (Moiseiwitsch, 1957). Total cross sections in units of $\pi a_{0}^{2}$.

\begin{tabular}{|c|c|c|c|c|c|}
\hline \multirow{2}{*}{$\begin{array}{c}\text { Impact }^{\mathrm{a}} \\
\text { energy } \\
(\mathrm{eV})\end{array}$} & \multicolumn{2}{|c|}{$2^{3} S \rightarrow 2^{3} P$} & \multirow[b]{2}{*}{$2^{3} S \rightarrow 3^{3} P$} & \multirow[b]{2}{*}{$2^{3} S \rightarrow 3^{3} D$} & \multirow[b]{2}{*}{$2^{3} S \rightarrow 4^{3} D$} \\
\hline & $\begin{array}{l}\text { First } \\
\text { Born }\end{array}$ & $\begin{array}{c}\text { Modified } \\
\text { Bethe }\end{array}$ & & & \\
\hline 1.14 & 0 & 0 & & & \\
\hline 1.22 & 173 & & & & \\
\hline 1.72 & & 79 & & & \\
\hline 2.18 & 294 & 94 & & & \\
\hline 3.40 & 248 & 110 & 1.08 & 3.60 & \\
\hline 4.89 & 202 & 114 & 1.29 & 6.94 & 1.69 \\
\hline 6.66 & 167 & 112 & 1.13 & 6.34 & 1.73 \\
\hline 8.70 & 138 & 105 & 1.07 & 5.41 & 1.51 \\
\hline 13.6 & 101 & 91 & 0.99 & 3.85 & 1.09 \\
\hline 19.6 & 76 & 77 & 0.91 & 2.81 & 0.80 \\
\hline 30.6 & 54 & 60 & 0.78 & 1.87 & 0.53 \\
\hline 54.4 & 34 & 41 & 0.59 & 1.08 & 0.31 \\
\hline 85.0 & 24 & 30 & 0.46 & 0.70 & 0.20 \\
\hline 122 & 18 & 23 & 0.37 & 0.49 & 0.14 \\
\hline
\end{tabular}

a The thresholds for the $2^{3} S \rightarrow 3^{3} P, 3^{3} D$, and $4^{3} D$ excitations occur at $3.19,3.25$, and $3.92 \mathrm{eV}$, respectively.

TABLE XXVI. Total cross sections for the excitation of the $2^{3} S$ state of helium calculated using the Born-Oppenheiner approximation and the first order exchange approximation (Bell; Eissa, and Moiseiwitsch, 1966). Total cross sections in units of $\pi a_{0}^{2}$. $^{\mathrm{a}}$

\begin{tabular}{ccc}
\hline $\begin{array}{c}\text { Impact energy } \\
\text { (rydbergs) }\end{array}$ & BO & $E_{1} \mathrm{c}$ \\
& & \\
1.44 & $9.52(-1)$ & $1.04(-1)$ \\
1.85 & $1.211(0)$ & $1.43(-1)$ \\
2.25 & $8.48(-1)$ & $9.79(-2)$ \\
2.56 & $6.23(-1)$ & $6.95(-2)$ \\
2.72 & $5.23(-1)$ & $5.81(-2)$ \\
3.24 & $3.17(-1)$ & $3.13(-2)$ \\
3.69 & $2.08(-1)$ & $1.89(-2)$ \\
4.00 & $1.59(-1)$ & $1.37(-2)$ \\
9.00 & $6.45(-3)$ & $7.35(-4)$ \\
16.00 & $3.63(-4)$ & $3.29(-4)$ \\
& & \\
\hline \hline
\end{tabular}




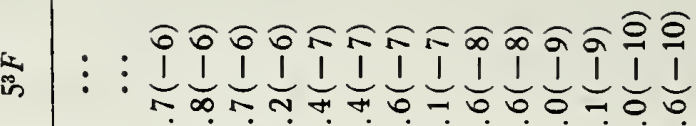

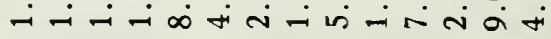

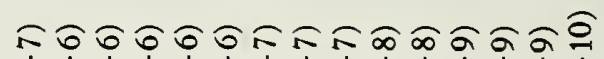

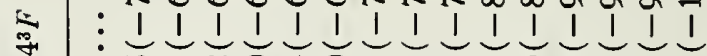
ทุก

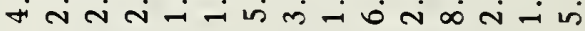

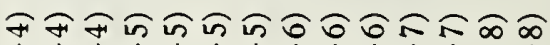

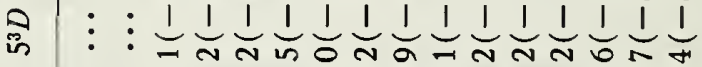

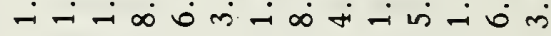

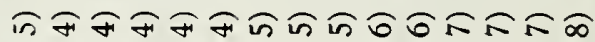
: : IIIIIIIIIIIIIII 等: :

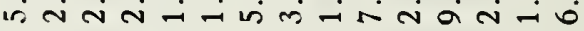

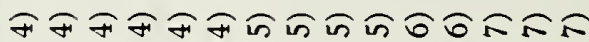
- : IIIIIIIIIIIIIII क : : तम

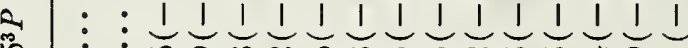
in : : U大a

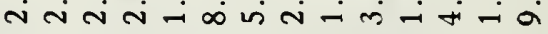

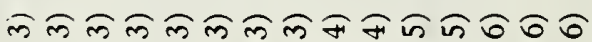

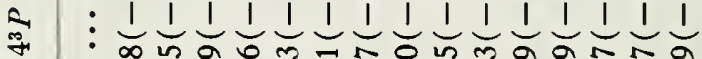

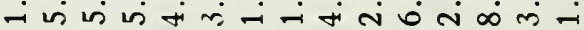

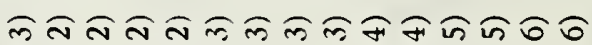

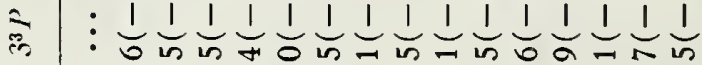

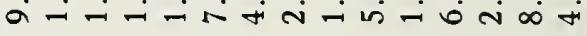

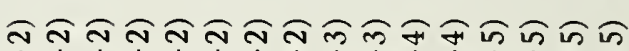

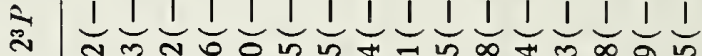

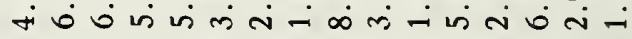

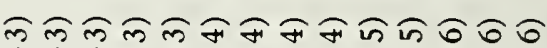

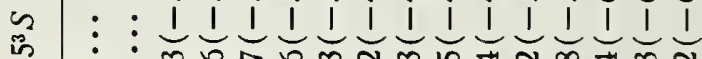

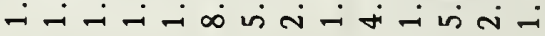

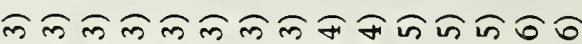

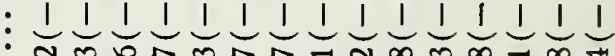

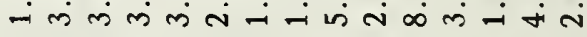

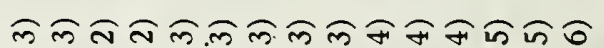

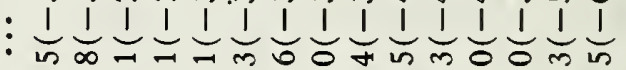

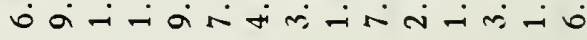

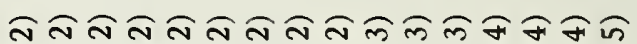

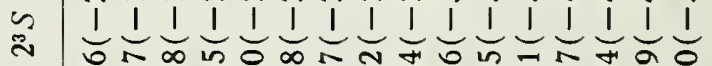
में 0 ठ 
TABLE XXIX. Total cross sections for the $1^{1} S \rightarrow n^{1} L$ excitations of helium calculated using the Ochkur approximation (Ochkur and Bratsev, 1965). Total cross sections in units of $\pi a_{0}^{2} .{ }^{2}$

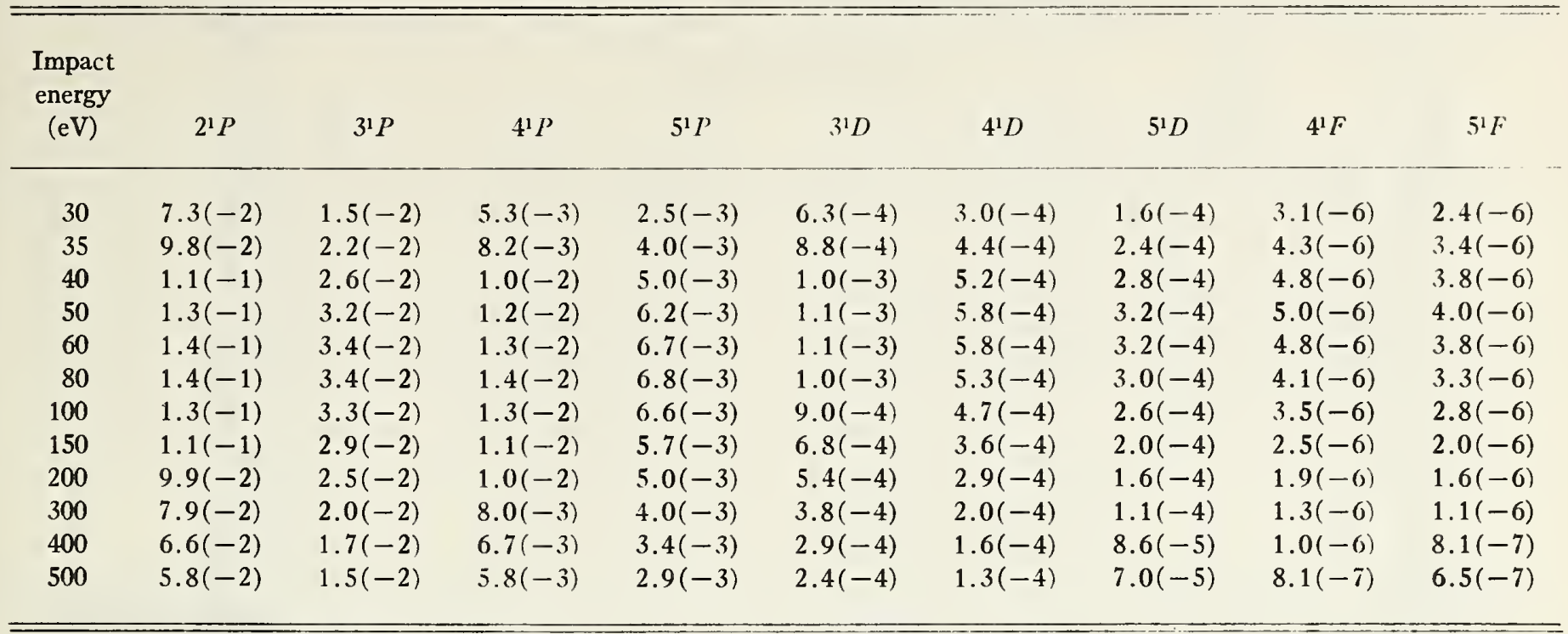

a The numbers in parentheses denote powers of 10 .

TABLE XXX. Total cross sections for the $2^{3} S \rightarrow n^{3} L$ excitations of helium calculated using the Ochkur approximation (Ochkur and Bratsev, 1966). Cross sections in units of $\pi a_{0}^{2}{ }^{*}$

Impact energy

$\begin{array}{lllllllllll}(\mathrm{eV}) & 3^{3} S & 4^{3} S & 5^{3} S & 7^{3} S & 10^{3} S & 3^{3} P & 4^{3} P & 5^{3} P & 7^{3} P & 10^{3} P\end{array}$

\begin{tabular}{rlllllllllll}
\hline & & & & & & & & & & \\
5 & $3.6(0)$ & $3.7(-1)$ & $\ldots$ & $\ldots$ & $\ldots$ & $1.6(0)$ & $1.8(-1)$ & $\ldots$ & $\ldots$ \\
5 & $3.6(0)$ & $5.8(-1)$ & $1.9(-1)$ & $4.5(-2)$ & $1.2(-2)$ & $1.5(0)$ & $3.4(-1)$ & $1.3(-1)$ & $3.3(-2)$ & $8.9(-3)$ \\
6 & $3.6(0)$ & $6.1(-1)$ & $2.1(-1)$ & $5.6(-2)$ & $1.6(-2)$ & $1.2(0)$ & $3.2(-1)$ & $1.3(-1)$ & $3.8(-2)$ & $1.2(-2)$ \\
8 & $3.2(0)$ & $5.8(-1)$ & $2.1(-1)$ & $5.7(-2)$ & $1.7(-2)$ & $9.8(-1)$ & $2.4(-1)$ & $1.0(-1)$ & $3.1(-2)$ & $9.6(-3)$ \\
10 & $2.8(0)$ & $5.2(-1)$ & $1.9(-1)$ & $5.3(-2)$ & $1.6(-2)$ & $9.5(-1)$ & $2.4(-1)$ & $1.0(-1)$ & $3.0(-2)$ & $9.4(-3)$ \\
15 & $2.1(0)$ & $4.0(-1)$ & $1.5(-1)$ & $4.2(-2)$ & $1.2(-2)$ & $8.0(-1)$ & $2.3(-1)$ & $1.0(-1)$ & $3.1(-2)$ & $9.9(-3)$ \\
20 & $1.7(0)$ & $3.2(-1)$ & $1.2(-1)$ & $3.4(-2)$ & $1.0(-2)$ & $7.8(-1)$ & $2.3(-1)$ & $1.0(-1)$ & $3.2(-2)$ & $1.0(-2)$ \\
30 & $1.2(0)$ & $2.3(-1)$ & $8.7(-2)$ & $2.4(-2)$ & $7.2(-3)$ & $7.3(-1)$ & $2.2(-1)$ & $9.5(-2)$ & $3.0(-2)$ & $9.6(-3)$ \\
40 & $9.0(-1)$ & $1.8(-1)$ & $6.7(-2)$ & $1.9(-2)$ & $5.6(-3)$ & $6.0(-1)$ & $1.9(-1)$ & $8.3(-2)$ & $2.7(-2)$ & $8.5(-3)$ \\
50 & $7.4(-1)$ & $1.4(-1)$ & $5.5(-2)$ & $1.5(-2)$ & $4.6(-3)$ & $5.5(-1)$ & $1.7(-1)$ & $7.7(-2)$ & $2.4(-2)$ & $7.9(-3)$ \\
70 & $5.4(-1)$ & $1.1(-1)$ & $4.0(-2)$ & $1.1(-2)$ & $3.3(-3)$ & $4.8(-1)$ & $1.5(-1)$ & $6.7(-2)$ & $2.1(-2)$ & $6.8(-3)$ \\
100 & $3.8(-1)$ & $7.5(-2)$ & $2.8(-2)$ & $7.8(-3)$ & $2.4(-3)$ & $4.3(-1)$ & $1.3(-1)$ & $5.8(-2)$ & $1.8(-2)$ & $5.9(-3)$
\end{tabular}

Impact energy

$\begin{array}{llllllllll}(\mathrm{eV}) & 3^{3} \mathrm{D} & 4^{3} \mathrm{D} & 5^{3} \mathrm{D} & 7^{3} \mathrm{D} & 10^{3} \mathrm{D} & 4^{3} \mathrm{~F} & 5^{3} \mathrm{~F} & 7^{3} \mathrm{~F} & 10^{3} \mathrm{~F}\end{array}$

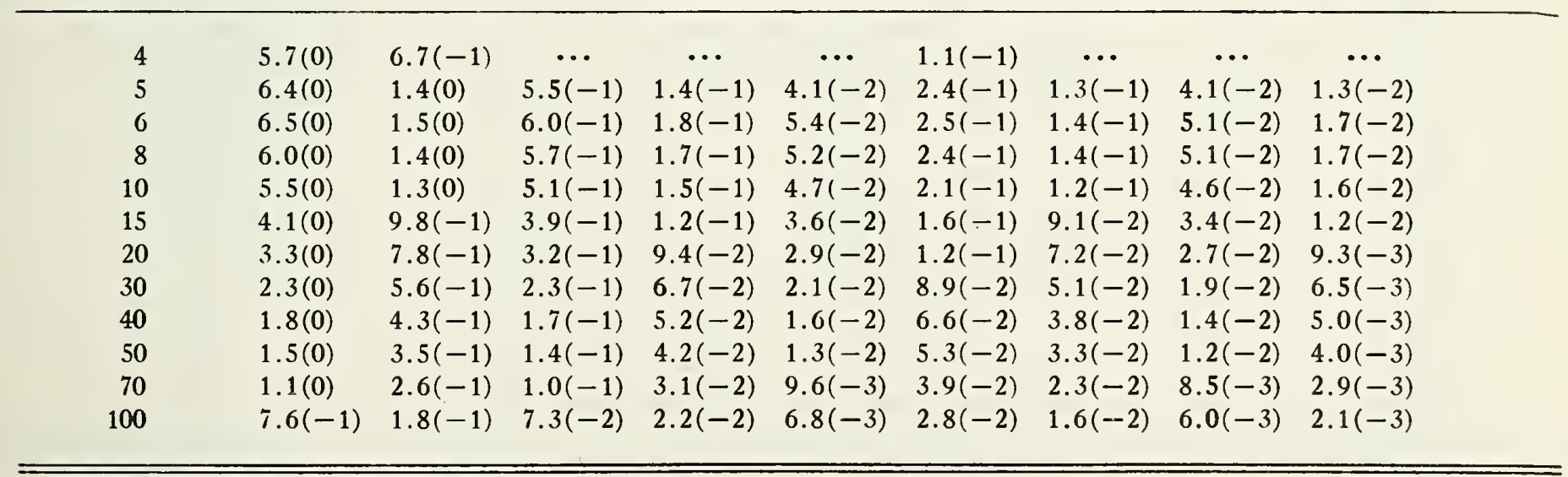

\footnotetext{
The numbers in parentheses denote powers of 10 .
} 


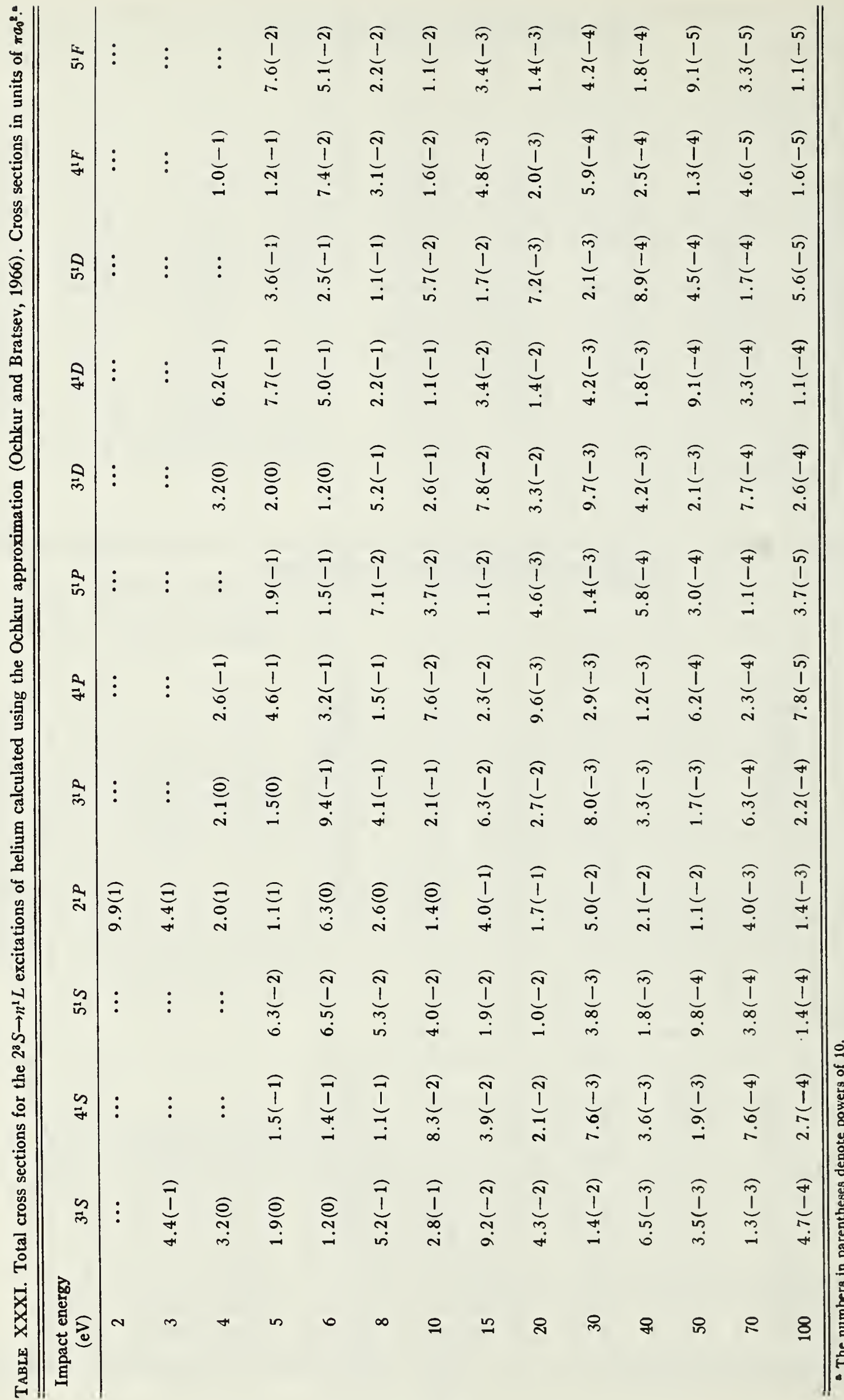


TABLE XXXV. Partial and total cross sections for the $1^{1} S \rightarrow 3^{1} P$ excitation of helium calculated using the $1^{1} S-3^{1} P$ close coupling approximation with exchange neglected (Vainshtein and Dolgov, 1959). Cross sections in units of $10^{-2} \pi a_{0}^{2}$.

\begin{tabular}{|c|c|c|c|c|c|c|c|}
\hline \multirow{2}{*}{$\begin{array}{c}\text { Impacta } \\
\text { energy } \\
(\mathrm{eV})\end{array}$} & \multicolumn{6}{|c|}{ Partial cross sections ${ }^{b}$} & \multirow{2}{*}{$\begin{array}{c}\text { Total } \\
\text { cross } \\
\text { sections }\end{array}$} \\
\hline & $Q^{10}$ & $Q^{01}$ & $Q^{21}$ & $Q^{12}$ & $Q^{32}$ & $Q^{23}$ & \\
\hline 23.342 & $4.46(-1)$ & $2.0(-4)$ & $1.72(-1)$ & $1.5(-6)$ & $4.6(-5)$ & .. & 0.649 \\
\hline 23.444 & $2.45(-1)$ & $4.3(-3)$ & $1.52(0)$ & $3.5(-6)$ & $5.6(-3)$ & $3.7(-6)$ & 1.808 \\
\hline 27.204 & $1.02(-1)$ & $3.66(-1)$ & $2.12(0)$ & $3.2(-3)$ & $1.135(0)$ & $4.7(-3)$ & 3.731 \\
\hline 32.012 & $7.4(-2)$ & $2.98(-1)$ & $1.52(0)$ & $2.8(-3)$ & $1.286(0)$ & $9.78(-3)$ & 3.191 \\
\hline 36.908 & $5.03(-2)$ & $2.33(-1)$ & $1.02(0)$ & $1.7(-3)$ & $1.220(0)$ & $1.39(-2)$ & 2.539 \\
\hline
\end{tabular}

\footnotetext{
a Threshold energy is $23.308 \mathrm{eV}$. b The numbers in parentheses denote powers of 10 .
}

TABLE XXXVI. Total cross sections for the double excitation of helium calculated using the first Born approximation (Massey and Mohr, 1935). Total cross sections in units of $\pi a_{0}{ }^{2} .^{\mathrm{a}}$

\begin{tabular}{cccccc}
\hline $\begin{array}{c}\text { Impact energy } \\
(\mathrm{eV})\end{array}$ & $(2 s)^{2}{ }^{1} S$ & $(2 s 2 p)^{1} P$ & $(2 s 3 p)^{1} P$ & $(2 s 4 p)^{1} P$ & $(3 s 2 p)^{1} P$ \\
\hline 75 & $1.1(-5)$ & $6.8(-4)$ & $3.3(-5)$ & $2.0(-5)$ & $1.5(-4)$ \\
100 & $1.7(-5)$ & $9.5(-4)$ & $5.2(-5)$ & $3.5(-5)$ & $3.5(-4)$ \\
200 & $2.4(-5)$ & $9.6(-4)$ & $6.8(-5)$ & $4.5(-5)$ & $5.3(-4)$ \\
300 & $2.8(-5)$ & $8.2(-4)$ & $6.2(-5)$ & $4.5(-5)$ & $5.8(-4)$ \\
400 & $3.0(-5)$ & $7.3(-4)$ & $5.3(-5)$ & $4.0(-5)$ & $5.4(-4)$ \\
600 & $3.2(-5)$ & $6.0(-4)$ & $4.4(-5)$ & $3.4(-5)$ & $5.0(-4)$ \\
\hline \hline
\end{tabular}

a The numbers in parentheses denote powers of 10 .

TABLE XXXVII. Total cross sections for the resonance transitions of the alkali atoms calculated using the first Born approximation (Vainshtein, Opykhtin, and Presnyakov, 1964b). ${ }^{\text {Total }}$ cross sections in units of $\pi a_{0}^{2}$.

\begin{tabular}{cccccc}
\hline \hline $\begin{array}{c}\text { Impact } \\
\text { energy } \epsilon \\
\text { in threshold } \\
\text { units }\end{array}$ & $\mathrm{Li}$ & $\mathrm{Na}$ & $\mathrm{K}$ & $\mathrm{Rb}$ & $\mathrm{Cs}$ \\
\hline & $2 s \rightarrow 2 p$ & $3 s \rightarrow 3 p$ & $4 s \rightarrow 4 p$ & $5 s \rightarrow 5 p$ & $6 s \rightarrow 6 p$ \\
1.02 & 41.4 & 30.7 & 54.7 & 58.5 & 75.0 \\
1.04 & 57.1 & 42.6 & 75.7 & 81.1 & 104 \\
1.08 & 77.1 & 57.8 & 103 & 110 & 141 \\
1.16 & 99.8 & 75.6 & 135 & 146 & 186 \\
1.32 & 120 & 93.2 & 166 & 180 & 230 \\
1.64 & 131 & 104 & 187 & 203 & 260 \\
2.28 & 124 & 103 & 186 & 202 & 260 \\
3.56 & 103 & 88.4 & 159 & 176 & 226 \\
6.12 & 75.3 & 66.6 & 120 & 133 & 172 \\
11.24 & 50.1 & 45.4 & 82.1 & 91.3 & 117 \\
21.48 & 31.2 & 28.7 & 52.0 & 58.0 & 74.6 \\
41.96 & 18.6 & 17.3 & 31.4 & 35.1 & 45.2 \\
& & & & & \\
\hline \hline
\end{tabular}

a Revised according to private communication from Professor Vainshtein (1966).
TABLE XXXVIII. Partial cross sections for the $3 s \rightarrow 3 p$ transition of sodium calculated using the $3 s-3 p$ close coupling approximation with exchange neglected. (Barnes, Lane, and Lin, 1965). Partial cross sections $Q^{\ell}$ in units of $\pi a_{0}^{2}$.

\begin{tabular}{crrrrr}
\hline \hline $\begin{array}{c}\text { Impact energy } \\
(\mathrm{eV})\end{array}$ & 4.210 & 7.364 & 10.520 & 16.832 & 23.144 \\
\hline$\ell$ & & & & & \\
0 & 0.01 & 0.72 & 0.94 & 0.54 & 0.33 \\
1 & 0.87 & 1.66 & 1.49 & 0.65 & 0.31 \\
2 & 15.96 & 6.02 & 2.62 & 0.99 & 0.52 \\
3 & 12.31 & 10.06 & 6.22 & 2.44 & 1.16 \\
4 & 7.94 & 9.52 & 7.16 & 3.72 & 2.03 \\
5 & 4.58 & 7.90 & 6.82 & 4.23 & 2.63 \\
6 & 2.48 & 6.13 & 5.98 & 4.21 & 2.88 \\
7 & 1.30 & 4.59 & 5.01 & 3.92 & 2.88 \\
8 & & 3.37 & 4.11 & 3.53 & 2.74 \\
9 & & 2.45 & 3.33 & 3.12 & 2.53 \\
10 & & & 2.68 & 2.73 & 2.30 \\
11 & & & 2.15 & 2.39 & 2.08 \\
12 & & & 1.73 & 2.08 & 1.88 \\
13 & & & & 1.81 & 1.69 \\
14 & & & & 1.57 & 1.52 \\
15 & & & & & 1.37 \\
\hline
\end{tabular}


TABLE XXXIX. Total cross sections for the $3 s \rightarrow 3 p$ transition of sodium calculated using the $3 s-3 p$ close coupling approximation with exchange neglected (Barnes, Lane, and Lin, 1965).

\begin{tabular}{cc|ccc}
\hline $\begin{array}{c}\text { Impact energy } \\
(\mathrm{eV})\end{array}$ & $\begin{array}{c}\text { Total cross } \\
\text { sections in units } \\
\text { of } \pi a_{0}^{2}\end{array}$ & $\begin{array}{c}\text { Total cross } \\
\text { Impact energy } \\
(\mathrm{eV})\end{array}$ & $\begin{array}{c}\text { sections in units } \\
\text { of } \pi a_{0}{ }^{2}\end{array}$ \\
\hline 4.210 & 46.8 & 16.832 & 48.9 \\
7.364 & 58.7 & 2.3 .144 & 42.1 \\
10.520 & 57.2 & & \\
\hline
\end{tabular}

Table XL. Total cross sections for the $6 s \rightarrow 6 p$ resonance transition of cesium calculated using the modified Bethe approximation (Hansen, 1964). Total cross sections in units of $\pi a_{0}^{2}$.

\begin{tabular}{cc|ccc}
\hline $\begin{array}{c}\text { Impact energy } \\
(\mathrm{eV})\end{array}$ & $Q_{6 p}$ & & $\begin{array}{c}\text { Impact energy } \\
(\mathrm{cV})\end{array}$ & $Q_{6 p}$ \\
\hline 1.60 & 41.56 & 5.03 & 114.3 \\
1.74 & 48.84 & 7.25 & 118.7 \\
1.89 & 61.27 & 11.60 & 114.6 \\
2.03 & 66.33 & 15.95 & 106.2 \\
2.18 & 70.16 & 23.20 & 92.60 \\
2.90 & 91.54 & 30.45 & 81.66 \\
\hline \hline
\end{tabular}

TABLE XLI. Total excitation cross sections for nonresonance transitions of lithium calculated using the first Born approximation

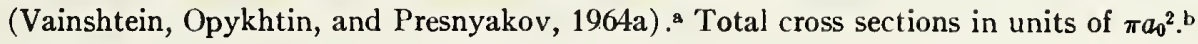
Impact energy $\epsilon$
in threshold units
$2 s \rightarrow 3 p \quad 2 s \rightarrow 4 p \quad 2 s \rightarrow 5 p \quad 2 s \rightarrow 3$
$2 s \rightarrow 3 d \quad 2 s \rightarrow 4 d \quad 2 s \rightarrow 5 d$
1.02
$9.67(-1) \quad 2.24(-1)$
$8.95(-2) \quad 1.21(0)$
$2.13(-1) \quad 7.81(-2) \quad 1.38(0)$
$4.09(-1) \quad 1.79(-1)$
1.04
$1.30(0) \quad 3.01(-1) \quad 1.20(-1) \quad 1.66(0)$
$2.91(-1) \quad 1.07(-1) \quad 1.90(0)$
$5.62(-1) \quad 2.45(-1)$
1.08
$1.68(0)$
$3.84(-1) \quad 1.52(-1) \quad 2.21(0)$
$3.86(-1) \quad 1.41(-1) \quad 2.56(0)$
$7.50(-1) \quad 3.26(-1)$
1.16
$2.01(0)$
$4.49(-1) \quad 1.76(-1) \quad 2.79(0)$
$4.84(-1) \quad 1.76(-1) \quad 3.31(0)$
$9.53(-1) \quad 4.10(-1)$
1.32
2.11(0)
$4.67(-1) \quad 1.77(-1) \quad 3.25(0)$
$5.55(-1) \quad 2.01(-1) \quad 3.96(0)$
$1.11(0)$
$4.73(-1)$
1.64
$1.87(0)$
$3.91(-1) \quad 1.50(-1) \quad 3.33(0)$
$5.58(-1) \quad 2.00(-1) \quad 4.19(0)$
$1.14(0) \quad 4.78(-1)$
2.28
$1.39(0)$
$2.84(-1) \quad 1.09(-1) \quad 2.90(0)$
$4.76(-1) \quad 1.60(-1) \quad 3.77(0)$
$9.86(-1) \quad 4.08(-1)$
3.56
$8.91(-1) \quad 1.84(-1) \quad 7.21(-2) \quad 2.12(0)$
$3.42(-1) \quad 1.21(-1) \quad 2.82(0)$
7. $16(-1) \quad 2.93(-1)$
6.12
$5.20(-1) \quad 1.13(-1) \quad 4.54(-2) \quad 1.34(0)$
$2.13(-1) \quad 7.50(-2) \quad 1.81(0)$
$4.50(-1) \quad 1.82(-1)$
11.24
$2.88(-1) \quad 6.67(-2) \quad 2.78(-2) \quad 7.68(0)$
$1.20(-1) \quad 4.23(-2) \quad 1.04(0)$
$2.56(-1) \quad 1.03(-1)$
21.48
$1.55(-1) \quad 3.85(-2) \quad 1.65(-2) \quad 4.12(0)$
$6.43(-2) \quad 2.25(-2) \quad 5.62(-1)$
$1.37(-1) \quad 5.50(-2)$
41.96
$8.23(-2) \quad 2.19(-2) \quad 9.63(-3) \quad 2.14(0)$
$3.32(-2) \quad 1.16(-2) \quad 2.92(-1) \quad 7.08(-2) \quad 2.85(-2)$

a Revised according to private communication from Professor Vainshtein (1966).

b The numbers in parentheses denote powers of 10 . 
TABLE XLIV. Total excitation cross sections for nonresonance transitions of rubidium calculated using the first Born approximation (Vainshtein, Opykhtin, and Presnyakov, 1964a). a Total cross sections in units of $\pi a_{0}^{2}$.b

\begin{tabular}{|c|c|c|c|c|c|c|c|c|c|c|}
\hline $\begin{array}{l}\text { Impact } \\
\text { nergy } \epsilon \text { in } \\
\text { threshold } \\
\text { units }\end{array}$ & $5 s \rightarrow 6 p$ & $5 s \rightarrow 7 p$ & $5 s \rightarrow 8 p$ & $5 s-6 s$ & $5 s \rightarrow 7 s$ & $5 s \rightarrow 8 s$ & $5 s \rightarrow+d$ & $5 s \rightarrow \overline{5} d$ & $5 s \rightarrow 6 d$ & $5 s \rightarrow 7 d$ \\
\hline 1.02 & $2.47(0)$ & $6.48(-1)$ & $2.79(-1)$ & $1.81(0)$ & $2.78(-1)$ & $1.06(-1)$ & $1.04(1)$ & $1.24(0)$ & $3.38(-1)$ & $1.23(-1)$ \\
\hline 1.04 & $3.37(0)$ & $8.84(-1)$ & $3.80(-1)$ & $2.51(0)$ & $3.85(-1)$ & $1.47(-1)$ & $1.43(1)$ & $1.67(0)$ & $4.65(-1)$ & $1.66(-1)$ \\
\hline 1.16 & $5.62(0)$ & $1.46(0)$ & $6.23(-1)$ & $4.38(0)$ & $6.81(-1)$ & $2.58(-1)$ & $2.35(1)$ & $2.58(0)$ & $6.89(-1)$ & $2.61(-1)$ \\
\hline 1.32 & $6.47(0)$ & $1.67(0)$ & $7.08(-1)$ & $5.27(0)$ & $8.25(-1)$ & $3.12(-1)$ & $2.60(1)$ & $2.71(0)$ & $7.11(-1)$ & $2.60(-1)$ \\
\hline 1.64 & $6.68(0)$ & $1.68(0)$ & $7.06(-1)$ & $5.64(0)$ & $8.81(-1)$ & $3.31(-1)$ & $2.62(1)$ & $2.42(0)$ & $6.18(-1)$ & $2.27(-1)$ \\
\hline 11.24 & $1.65(0)$ & $3.98(-1)$ & $1.50(-1)$ & $1.42(0)$ & $2.10(-1)$ & $7.67(-2)$ & $5.36(0)$ & $3.72(-1)$ & $9.29(-2)$ & $3.66(-2)$ \\
\hline 21.48 & $9.29(-1)$ & $2.22(-1)$ & $8.86(-2)$ & $7.60(-1)$ & $1.13(-1)$ & $4.10(-2)$ & $2.86(0)$ & $1.95(-1)$ & $4.88(-2)$ & $1.93(-2)$ \\
\hline 41.96 & $5.08(-1)$ & $1.20(-1)$ & $4.47(-2)$ & $3.98(-1)$ & $5.85(-2)$ & $2.12(-2)$ & $1.48(0)$ & $9.98(-2)$ & $2.50(-2)$ & $9.97(-3)$ \\
\hline
\end{tabular}

${ }^{8}$ Revised according to private communication from Professor Vainshtein (1966).

b The numbers in parentheses denote powers of 10 .

TABLE XLV. Total excitation cross sections for nonresonance transitions of cesium calculated using the first Born approximation

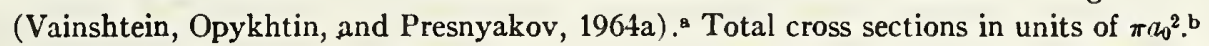

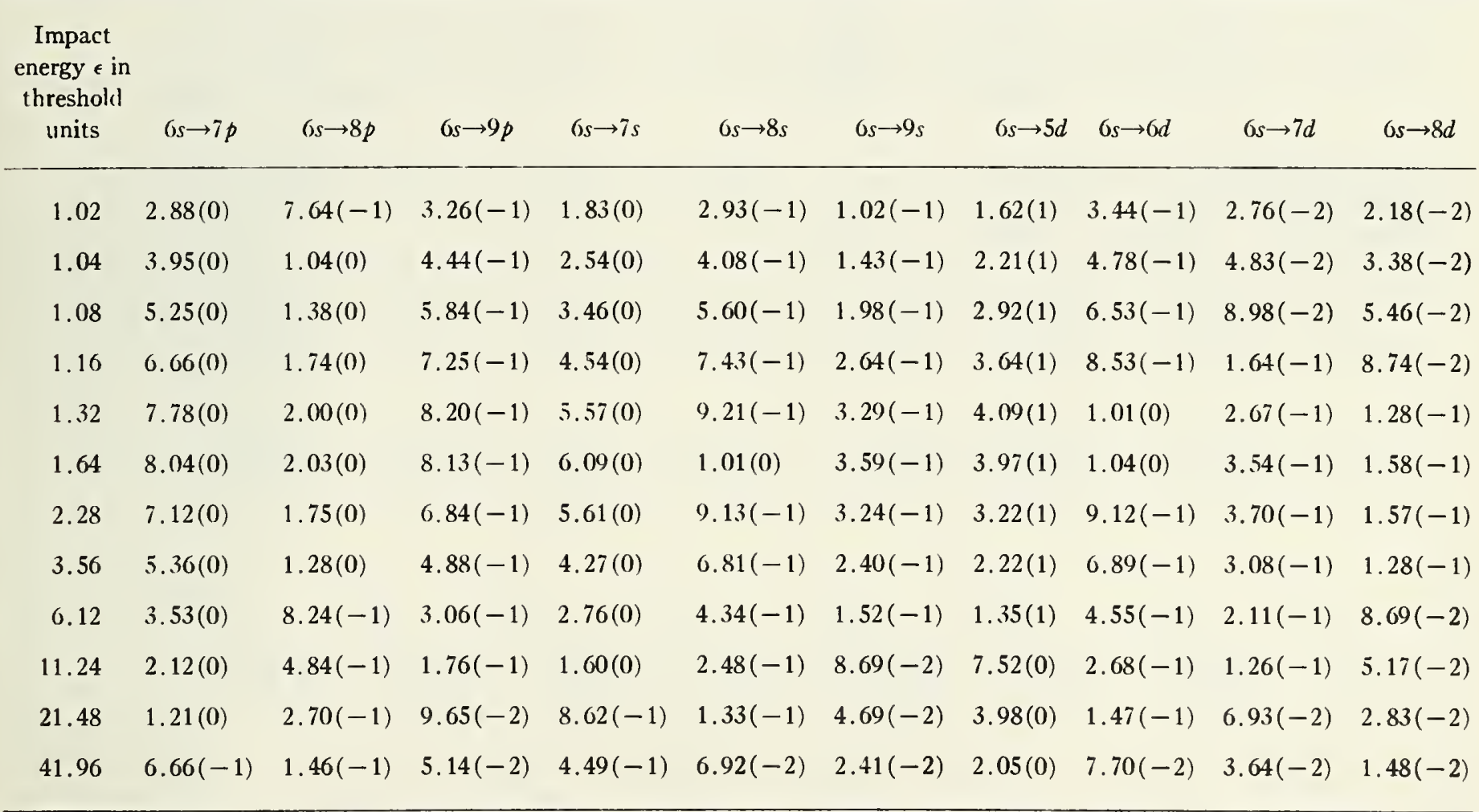


TABLE XLVI. Collision strengths for the ${ }^{3} P \rightarrow^{1} D$ excitation of oxygen calculated for $k_{1}{ }^{2}=0.3$ (Seaton, 1953a).

\begin{tabular}{cccc}
\hline Approximation & $s^{p}(1,2)$ & $\varsigma^{p}(2,1)$ & $\widetilde{\Omega}^{p}(1,2)$ \\
\hline I & 0.48 & 0.48 & 0.48 \\
II & 0.81 & 0.62 & 0.71 \\
III & 0.98 & 0.88 & 0.93 \\
IV & 1.06 & 0.90 & 0.98 \\
\hline
\end{tabular}

TABLE XLVII. Collision strengths for transitions connecting the ${ }^{3} P,{ }^{1} D$, and ${ }^{1} S$ terms of the lowest configuration of atomic oxygen (Seaton, 1955b; 1956).

\begin{tabular}{cccc}
\hline \hline $\begin{array}{c}k_{n}{ }^{2} \\
\text { (a.u. })\end{array}$ & $\begin{array}{c}\Omega(1,2) \\
(n=2)\end{array}$ & $\begin{array}{c}\Omega(1,3) \\
(n=3)\end{array}$ & $\begin{array}{c}\Omega(2,3) \\
(n=3)\end{array}$ \\
\hline 0.000 & 0.00 & 0.000 & 0.000 \\
0.025 & 0.15 & 0.017 & 0.009 \\
0.050 & 0.34 & 0.050 & 0.024 \\
0.075 & 0.53 & 0.075 & 0.040 \\
0.100 & 0.69 & 0.100 & 0.057 \\
0.150 & 0.97 & 0.144 & 0.088 \\
0.163 & 1.04 & $\ldots .18$ & $\ldots .18$ \\
0.2 & 1.06 & 0.180 & 0.118 \\
0.3 & 1.20 & 0.238 & 0.167 \\
0.4 & 1.32 & 0.278 & 0.208 \\
0.5 & 1.44 & 0.305 & 0.240 \\
0.6 & 1.53 & 0.324 & 0.269 \\
0.7 & 1.60 & 0.335 & 0.295 \\
0.8 & 1.67 & 0.344 & 0.316 \\
0.9 & 1.73 & 0.349 & 0.336 \\
1.0 & 1.77 & 0.353 & 0.354 \\
To obtain N collision & & & \\
strengths multiply & & & \\
O strengths by & $25 / 24$ & $25 / 8$ & $225 / 16$ \\
\hline \hline
\end{tabular}

TABLE XLVIII. Deactivation coefficients for atomic oxygen and nitrogen (Seaton, 1955b; 1956). Deactivation coefficients for $\mathrm{O}$ and $\mathrm{N}\left(\alpha_{n n^{\prime}}\right.$ in units of $\left.\mathrm{cm}^{3} \mathrm{sec}^{-1}\right)$.

\begin{tabular}{ccccccc}
\hline $\begin{array}{c}\text { Electron } \\
\text { tempera- } \\
\text { ture } \\
T\end{array}$ & $10^{9} \alpha_{21}$ & $10^{9} \alpha_{31}$ & $10^{9} \alpha_{32}$ & & & \\
$10^{9} \alpha_{21}$ & $10^{9} \alpha_{31}$ & $10^{9} \alpha_{32}$ \\
\hline $5 \times 10^{2}$ & 0.9 & 0.8 & 0.4 & 0.5 & 0.4 & 0.9 \\
$1 \times 10^{3}$ & 1.6 & 1.2 & 0.6 & 0.8 & 0.6 & 1.5 \\
$5 \times 10^{3}$ & 5.0 & 3.5 & 1.9 & 2.6 & 1.8 & 4.4 \\
$1 \times 10^{4}$ & 0.7 & 5.1 & 3.0 & 3.5 & 2.7 & 0.9 \\
$5 \times 10^{4}$ & 7.6 & 7.2 & 5.6 & 4.0 & 3.7 & 13 \\
$1 \times 10^{5}$ & 6.8 & 6.6 & 6.0 & 3.5 & 3.4 & 14 \\
\hline \hline
\end{tabular}

TABLE XLIX. Effective Kramers Gaunt factor $\vec{g}$ for the $1 s \rightarrow 2 p$ excitation of hydrogenic ions (Burgess, 1961).

\begin{tabular}{ccc}
\hline & \multicolumn{3}{c}{$Z$} \\
$\begin{array}{c}\text { Impact energy } \epsilon \\
\text { in threshold units }\end{array}$ & 2 & $\infty$ \\
1 & 0.155 & 0.21 \\
$4 / 3$ & 0.21 & 0.25 \\
$8 / 3$ & 0.37 & 0.38 \\
4 & 0.45 & 0.46 \\
$16 / 3$ & 0.53 & 0.54 \\
\hline
\end{tabular}




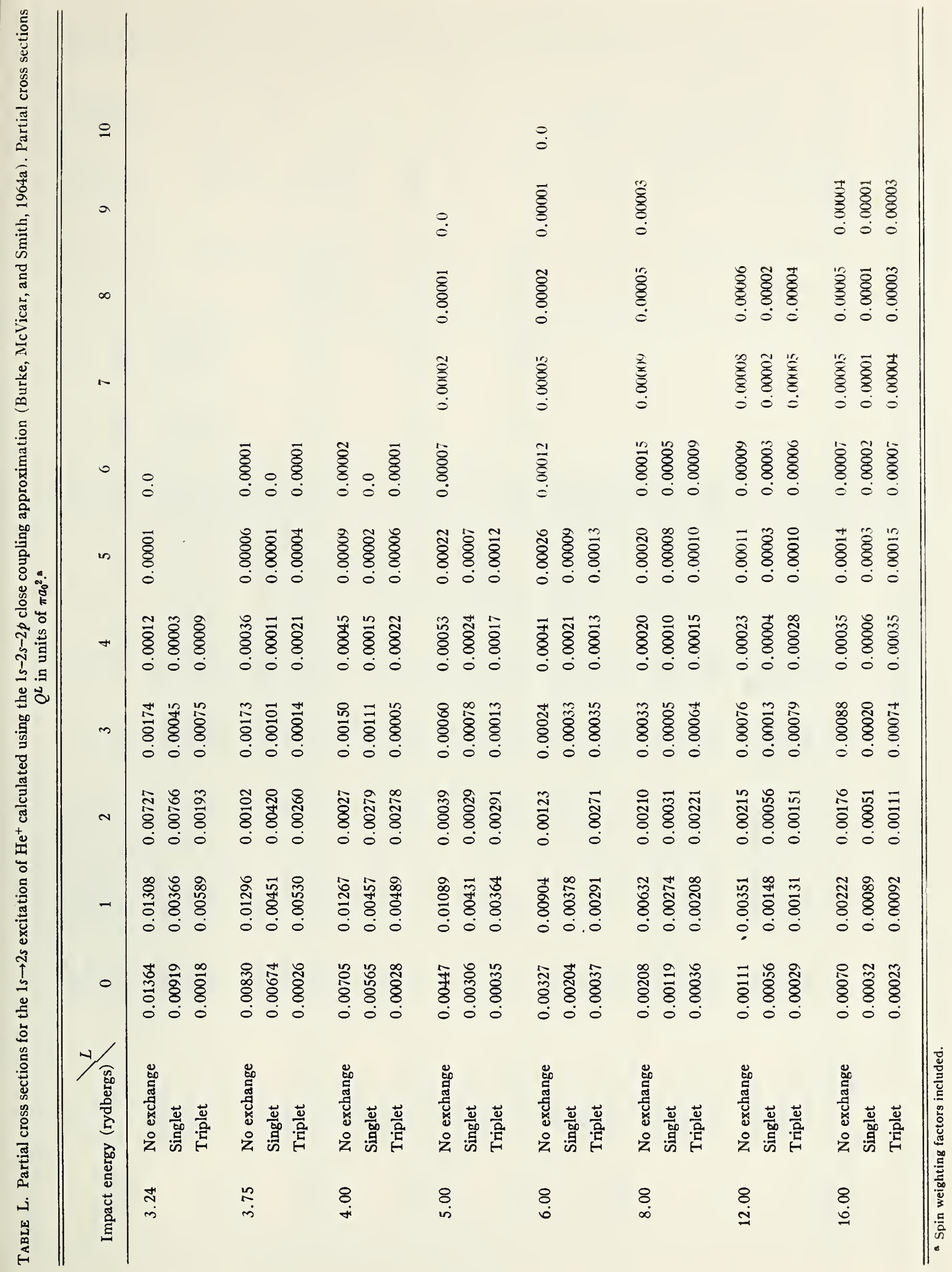




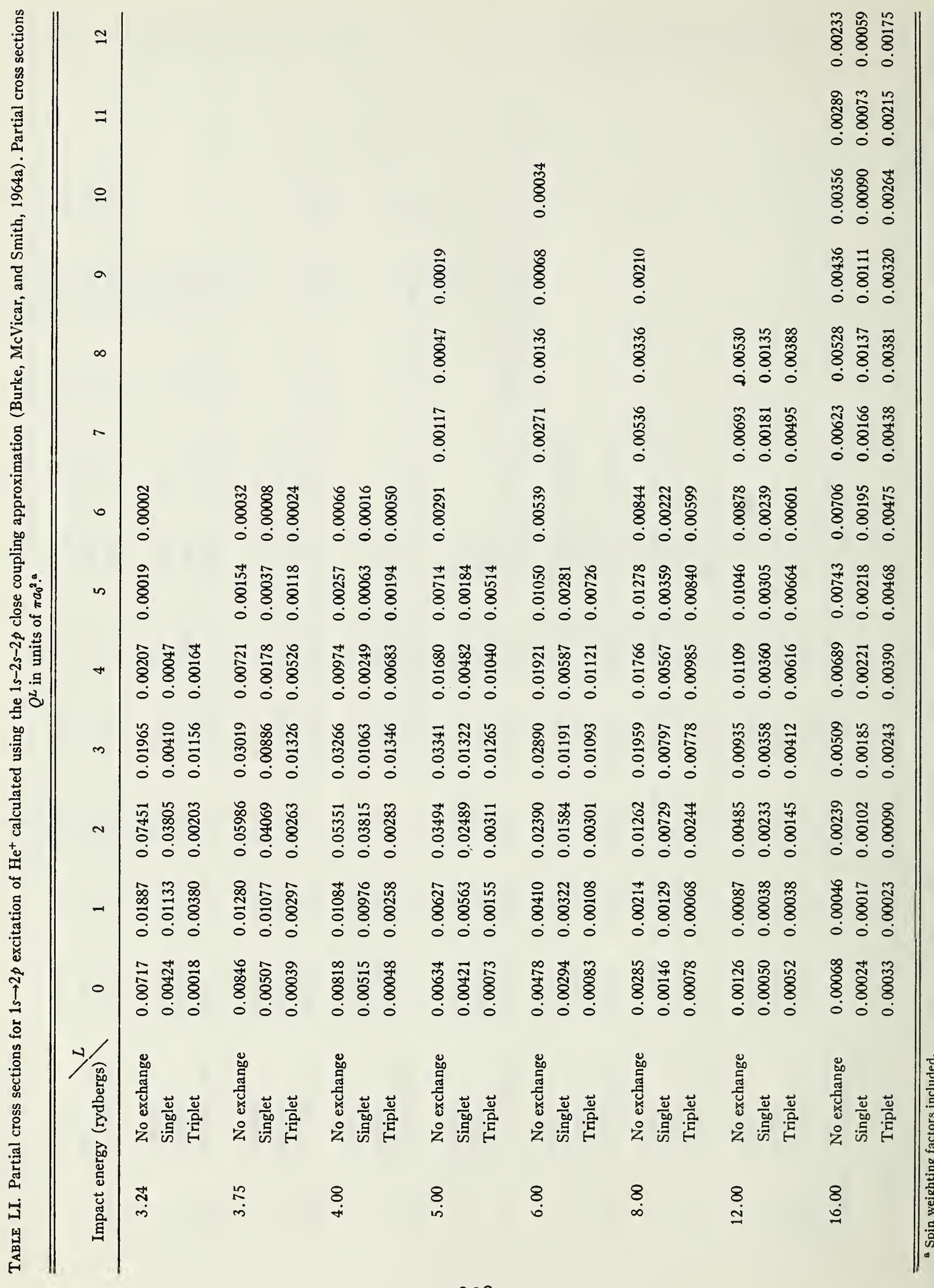




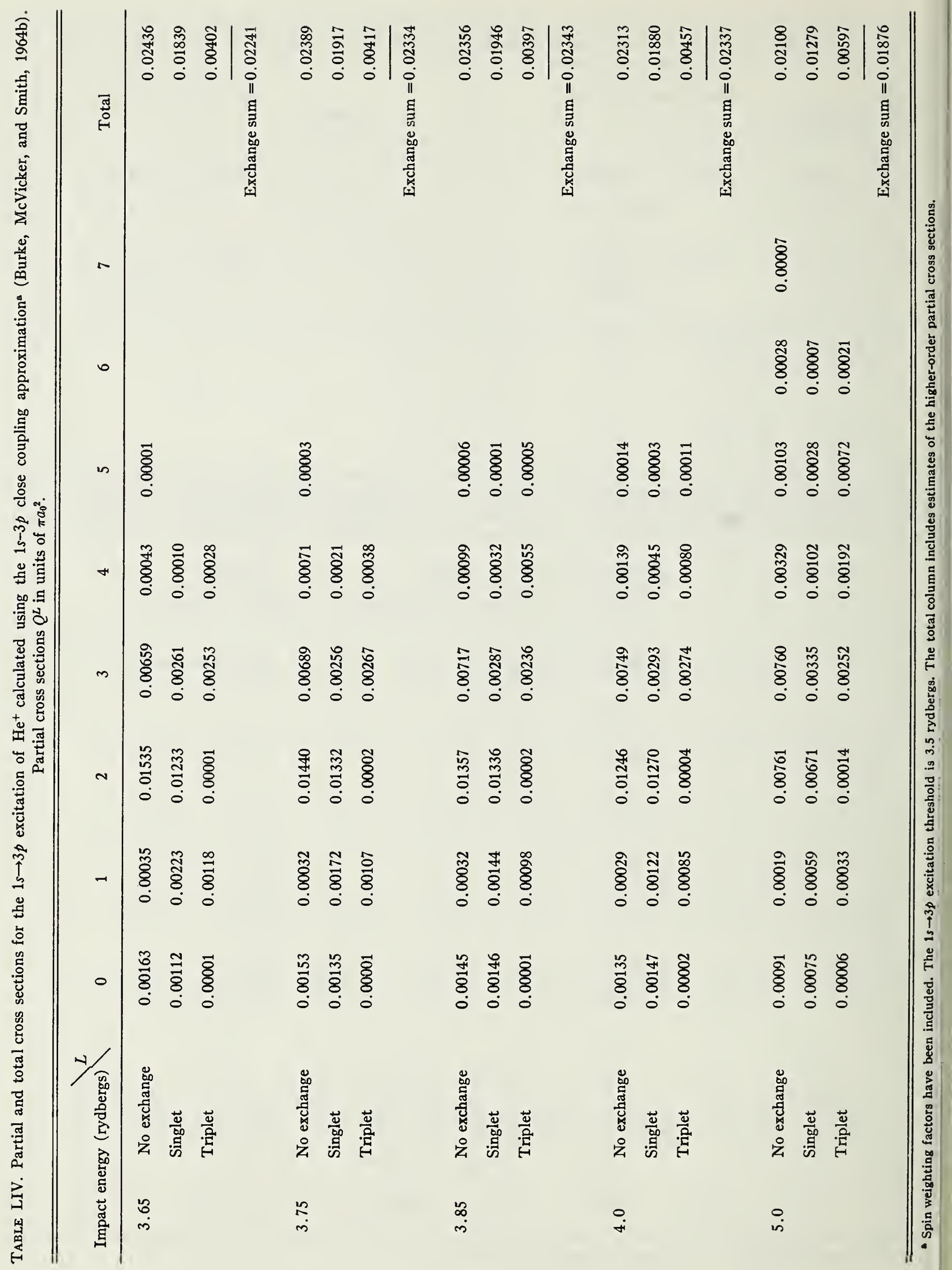


TABLE LV. Total excitation cross sections for transitions of $\mathrm{N}^{4+}$ calculated using the Coulomb-Born approximation (Burke, Tait, and Lewis, 1966). Cross sections in units of $\pi a_{0}^{2}$.

\begin{tabular}{|c|c|c|c|c|c|c|c|c|c|c|}
\hline $\begin{array}{l}\text { Incident electron } \\
\text { energy (rydbergs) }\end{array}$ & $2 s \rightarrow 2 p$ & $2 s \rightarrow 3 s$ & $2 s \rightarrow 3 p$ & $2 s \rightarrow 3 d$ & $2 p \rightarrow 3 s$ & $2 p \rightarrow 3 p$ & $2 p \rightarrow 3 d$ & $3 s \rightarrow 3 p$ & $3 s \rightarrow 3 d$ & $3 p \rightarrow 3 d$ \\
\hline 1.0 & 3.87 & & & & & & & & & \\
\hline 1.44 & 2.80 & & & & & & & & & \\
\hline 2.25 & 1.92 & & & & & & & & & \\
\hline 4.0 & 1.20 & & & & & & & & & \\
\hline 4.5455 & & & & & & & & & & 76 \\
\hline 5.0 & 1.01 & 0.0304 & 0.0097 & 0.0519 & 0.00316 & 0.0657 & 0.190 & 31.5 & 2.73 & 36.4 \\
\hline 6.0 & 0.87 & 0.0257 & 0.0104 & 0.0462 & 0.00318 & 0.0551 & 0.172 & 17.0 & 1.25 & 15.4 \\
\hline 8.0 & 0.69 & 0.0197 & 0.0112 & 0.0381 & 0.00311 & 0.0420 & 0.148 & 9.4 & 0.592 & 9.17 \\
\hline 12 & 0.50 & 0.0134 & 0.0114 & 0.0286 & 0.00287 & 0.0287 & 0.120 & 5.23 & 0.283 & 4.19 \\
\hline 16 & 0.42 & 0.0101 & 0.0110 & 0.0230 & 0.00263 & 0.0220 & 0.104 & 3.51 & 0.185 & 2.35 \\
\hline 24 & 0.29 & 0.0068 & 0.0099 & 0.0164 & 0.00224 & 0.0150 & 0.084 & 2.12 & 0.106 & 1.35 \\
\hline 32 & 0.23 & 0.0051 & 0.0089 & 0.0128 & 0.00196 & 0.0114 & 0.071 & 1.65 & 0.072 & 0.94 \\
\hline
\end{tabular}

The incident electron energy is given relative to the $(1 s)^{2} 2 s$ ground state.

TABle LVI. Total excitation cross sections for the $4 s \rightarrow 4 p, 3 d \rightarrow 4 p$, and $4 s \rightarrow 3 d$ transitions of $\mathrm{Ca}^{+}$calculated using the Coulomb-Born approximation and the unitarized Coulomb-Born approximation (Van Regemorter, 1960a; 1961). Total cross sections in units of $\pi a_{0}{ }^{2}$

\begin{tabular}{|c|c|c|c|c|c|c|}
\hline \multirow[b]{2}{*}{$k_{f}^{2}$} & \multicolumn{2}{|c|}{$4 s \rightarrow 4 p^{\mathrm{a}}$} & \multicolumn{2}{|c|}{$3 d \rightarrow 4 p^{2}$} & \multicolumn{2}{|c|}{$4 s \rightarrow 3 d^{b}$} \\
\hline & 0 & 0.1 & 0 & 0.1 & 0.1062 & 0.2062 \\
\hline Coulomb-Born & 126.5 & 99.7 & 94.6 & 47.1 & 17.35 & 16.38 \\
\hline $\begin{array}{l}\text { Unitarized Coulomb- } \\
\text { Born }\end{array}$ & 58.2 & 52.0 & 58.3 & 31.5 & 10.8 & 10.2 \\
\hline
\end{tabular}

Van Regemorter, 1960a. b Van Regemorter, 1961.

TABLE LVII. Collision strengths for the excitation of forbidden transitions between the terms of the lowest $p^{2}, p^{3}$, and $p^{4}$ configurations of certain positive ions (Seaton, 1953b; 1955b 1956). .

\begin{tabular}{|c|c|c|c|c|}
\hline Configuration & Ion & $\Omega(1,2)$ & $\Omega(1,3)$ & $\Omega(2,3)$ \\
\hline \multirow[t]{4}{*}{$2 p^{2}$} & $\mathrm{~N}^{+}$ & 2.39 & 0.223 & 0.46 \\
\hline & $\mathrm{O}^{2+}$ & 1.73 & 0.195 & 0.61 \\
\hline & $\mathrm{F}^{3+}$ & 1.21 & 0.172 & 0.58 \\
\hline & $\mathrm{Ne}^{4+}$ & 0.84 & 0.157 & 0.53 \\
\hline \multirow[t]{4}{*}{$2 p^{3}$} & $\mathrm{O}^{+}$ & 1.44 & 0.218 & 1.92 \\
\hline & $\mathrm{F}^{2+}$ & 1.00 & 0.221 & 3.11 \\
\hline & $\mathrm{Ne}^{3+}$ & 0.68 & 0.234 & 3.51 \\
\hline & $\mathrm{Na}^{4+}$ & 0.43 & 0.255 & 3.49 \\
\hline \multirow[t]{4}{*}{$2 p^{4}$} & $\mathrm{~F}^{+}$ & 0.95 & 0.057 & 0.17 \\
\hline & $\mathrm{Ne}^{2+}$ & 0.76 & 0.077 & 0.27 \\
\hline & $\mathrm{Na}^{8+}$ & 0.61 & 0.092 & 0.30 \\
\hline & $\mathrm{Mg}^{4+}$ & 0.54 & 0.112 & 0.30 \\
\hline $3 p^{3}$ & $\mathrm{~S}^{+}$ & 2.02 & 0.383 & 12.7 \\
\hline
\end{tabular}

TABLE LVIII. Values of the constants $G, h_{0}, h_{1}, h_{2}$ occurring in the polarization formulas for the helium lines $L S J \rightarrow J^{\prime \prime}$ (Percival and Seaton, 1958).

\begin{tabular}{|c|c|c|c|c|c|}
\hline$S L J$ & $J^{\prime \prime}$ & $G$ & $h_{0}$ & $h_{1}$ & $h_{2}$ \\
\hline \multirow[t]{3}{*}{011} & 0 & 1 & 1 & 1 & $\cdots$ \\
\hline & 1 & -1 & 1 & 3 & ... \\
\hline & 2 & 1 & 7 & 13 & $\cdots$ \\
\hline \multirow[t]{3}{*}{022} & 1 & 3 & 5 & 9 & 6 \\
\hline & 2 & -3 & 3 & 7 & 10 \\
\hline & 3 & 3 & 15 & 29 & 26 \\
\hline 110 & 1 & 0 & ... & . & $\cdots$ \\
\hline \multirow[t]{3}{*}{111} & 0 & -1 & 1 & 3 & ... \\
\hline & 1 & 1 & 3 & 5 & $\cdots$ \\
\hline & 2 & -1 & 13 & 27 & $\cdots$ \\
\hline \multirow[t]{3}{*}{112} & 1 & 21 & 47 & 73 & ... \\
\hline & 2 & -7 & 11 & 29 & $\cdots$ \\
\hline & 3 & 1 & 7 & 13 & .. \\
\hline \multirow[t]{3}{*}{121} & 0 & 3 & 5 & 9 & 6 \\
\hline & 1 & -3 & 7 & 15 & 18 \\
\hline & 2 & 3 & 41 & 81 & 78 \\
\hline \multirow[t]{3}{*}{122} & 1 & 3 & 9 & 17 & 14 \\
\hline & 2 & -3 & 7 & 15 & 18 \\
\hline & 3 & 3 & 29 & 57 & 54 \\
\hline \multirow[t]{3}{*}{123} & 2 & 18 & 41 & 76 & 58 \\
\hline & 3 & -9 & 11 & 25 & 34 \\
\hline & 4 & 3 & 15 & 29 & 26 \\
\hline
\end{tabular}


TABLE LIX. Values of the constants $G, h_{0}, h_{1}, h_{2}$ occurring in the polarization formulas for the helium multiplets $L S \rightarrow L^{\prime \prime} S$ (Percival and Seaton, 1958).

\begin{tabular}{crrrrr}
\hline$S L$ & $L^{\prime \prime}$ & $G$ & \multicolumn{1}{c}{$h_{0}$} & $h_{1}$ & $h_{2}$ \\
\hline 01 & 0 & 1 & 1 & 1 & $\cdots$ \\
& 2 & 1 & 7 & 13 & $\cdots$ \\
02 & 1 & 3 & 5 & 9 & 6 \\
& 3 & 3 & 15 & 29 & 26 \\
11 & 0 & 15 & 41 & 67 & $\cdots$ \\
& 2 & 3 & 73 & 143 & $\cdots$ \\
& & & & & \\
& 1 & 213 & 671 & 1271 & 1058 \\
& 3 & 213 & 2171 & 4271 & 4058 \\
\hline \hline
\end{tabular}

TABLE LX. Values of the constants $G, h_{0}, h_{1}$ occurring in the polarization formula for the ${ }^{2} P_{3 / 2} \rightarrow{ }^{2} S$ transition (Percival and Seaton, 1958).

\begin{tabular}{rrrr}
\hline \hline$I$ & $G$ & \multicolumn{1}{c}{$h_{0}$} & \multicolumn{1}{c}{$h_{1}$} \\
\hline 0 & 3 & 5 & 7 \\
$\frac{1}{2}$ & 15 & 37 & 59 \\
1 & 33 & 161 & 289 \\
$\frac{3}{2}$ & 81 & 427 & 773 \\
\hline
\end{tabular}

TABLE LXI. Values of the constants $G, h_{0}, h_{1}$ occurring in the polarization formula for the ${ }^{2} P \rightarrow{ }^{2} S$ multiplet (Percival and Seaton, 1958).

\begin{tabular}{rrrr}
\hline$I$ & $G$ & \multicolumn{1}{c}{$h_{0}$} & \multicolumn{1}{c}{$h_{1}$} \\
\hline 0 & 3 & 7 & 11 \\
$\frac{1}{2}$ & 15 & 53 & 91 \\
1 & 33 & 236 & 439 \\
$\frac{3}{2}$ & 27 & 209 & 391 \\
\hline
\end{tabular}

TABLE LXII. Threshold percentage polarization for resonance lines of lithium and sodium.

\begin{tabular}{|c|c|c|}
\hline Ion & Calculated` & Measured $^{b}$ \\
\hline${ }^{6} \mathrm{Li}$ & 37.5 & $39.7 \pm 3.8$ \\
\hline${ }^{7} \mathrm{Li}$ & 21.6 & $20.6 \pm 3.0$ \\
\hline${ }^{23} \mathrm{Na}$ & 14.1 & $14.8 \pm 1.8$ \\
\hline \multicolumn{3}{|c|}{$\begin{array}{l}\text { Flower and Seaton, } 1967 . \\
\text { Hafner, Kleinpoppen, and Kruger, } 1965 .\end{array}$} \\
\hline
\end{tabular}

TABLE LXIII. Percentage polarization of the $2 p \rightarrow 1 s$ multiplet of atomic hydrogen calculated using the $1 s-2 s-2 p$ close coupling approximation $1 s \rightarrow 2 p$ excitation cross sections.

\begin{tabular}{lll}
\hline \hline$k_{2 p^{2}}$ & $P(\%)$ \\
\hline $0.01^{\mathrm{a}}$ & 17.3 \\
$0.03^{\mathrm{a}}$ & 15.7 \\
$0.05^{\mathrm{a}}$ & 16.7 \\
$0.06^{\mathrm{b}}$ & 20.9 \\
$0.25^{\mathrm{b}}$ & 28.35 \\
$0.46^{\mathrm{b}}$ & 27.99 \\
$0.69^{\mathrm{b}}$ & 26.67 \\
$1.50^{\mathrm{b}}$ & 18.00 \\
$3.25^{\mathrm{b}}$ & 8.55 \\
\hline \hline
\end{tabular}


(a) Helium $n^{1} P \rightarrow 2^{1} S$

\begin{tabular}{|c|c|c|c|c|c|c|}
\hline$n^{1} P$ & $\begin{array}{c}\text { Branching } \\
\quad \text { ratio } \\
B(n \rightarrow 2)\end{array}$ & $\begin{array}{c}Q(n \rightarrow 2) \\
\left(10^{-20} \mathrm{~cm}^{2}\right)\end{array}$ & $\begin{array}{c}Q\left(n^{1} P\right) \\
=Q(n \rightarrow 2) / B(n \rightarrow 2) \\
\left(\mathrm{i}^{-20} \mathrm{~cm}^{2}\right)\end{array}$ & $\begin{array}{l}\text { Electron } \\
\text { energy } \\
(\mathrm{eV})\end{array}$ & Reference & Comments \\
\hline $31 P$ & 0.0231 & 17.4 & $\begin{array}{l}150 \\
457 \\
350 \\
750 \\
260 \\
290\end{array}$ & $\begin{array}{l}90-110 \\
108 \\
100 \\
100-110 \\
100 \\
100\end{array}$ & $\begin{array}{l}\mathrm{s} \\
\mathrm{u} \\
\mathrm{v} \\
\mathrm{w} \\
\mathrm{x} \\
\mathrm{y}\end{array}$ & $\begin{array}{r}\mathrm{cfg} \\
\mathrm{f} \mathrm{k} \\
\mathrm{adeg} \\
\mathrm{adg} \\
\mathrm{ah} \mathrm{jl} \\
\text { Theoretical, } \mathrm{m}\end{array}$ \\
\hline $4^{1} P$ & 0.0281 & 8.5 & $\begin{array}{c}53 \\
210 \\
159 \\
300 \\
89.2 \\
114.5\end{array}$ & $\begin{array}{l}100-120 \\
108 \\
100 \\
100-120 \\
100 \\
100\end{array}$ & $\begin{array}{l}s \\
u \\
v \\
w \\
x \\
y\end{array}$ & $\begin{array}{r}\text { c f g } \\
\mathrm{f} \mathrm{k} \\
\mathrm{bdeg} \\
\mathrm{adg} \mathrm{i} \\
\text { a h j l } \\
\text { Theoretical, } \mathrm{m}\end{array}$ \\
\hline $5^{1} P$ & 0.0283 & 3.9 & $\begin{array}{c}84 \\
140 \\
45.2 \\
58.1\end{array}$ & $\begin{array}{l}108 \\
100-120 \\
100 \\
100\end{array}$ & $\begin{array}{l}u \\
w \\
x \\
y\end{array}$ & $\begin{array}{r}\mathrm{f} \mathrm{k} \\
\mathrm{a} \mathrm{d} \mathrm{g} \mathrm{i} \\
\mathrm{a} \mathrm{h} \mathrm{j} \mathrm{l} \\
\text { Theoretical, } \mathrm{m}\end{array}$ \\
\hline $6^{1} P$ & 0.032 & 2.3 & $\begin{array}{l}39 \\
72\end{array}$ & $\begin{array}{l}108 \\
100-120\end{array}$ & $\begin{array}{l}u \\
w\end{array}$ & $\begin{array}{r}f k \\
a d g i\end{array}$ \\
\hline $7^{1} P$ & $\sim 0.025$ & $\sim 0.7$ & $\sim 30$ & $100-120$ & $\mathbf{w}$ & $\mathrm{adg} i$ \\
\hline
\end{tabular}

(b) Helium $n^{1} S \rightarrow 2^{1} P$

\begin{tabular}{|c|c|c|c|c|c|c|c|}
\hline$n^{1} S$ & $\begin{array}{l}\text { Branching } \\
\quad \text { ratio } \\
B(n \rightarrow 2)\end{array}$ & $\begin{array}{c}Q(n \rightarrow 2) \\
\left(10^{-20} \mathrm{~cm}^{2}\right)\end{array}$ & $\begin{array}{c}Q\left(n^{1} S\right) \\
=Q(n \rightarrow 2) / B(n \rightarrow 2) \\
\left(10^{-20} \mathrm{~cm}^{2}\right)\end{array}$ & $\begin{array}{r}\text { Electron } \\
\text { energy } \\
(\mathrm{eV})\end{array}$ & $\begin{array}{c}Q\left(n^{1} S\right) \\
\text { Translated } \\
\text { to } 100 \mathrm{eV} \\
\left(10^{-20} \mathrm{~cm}^{2}\right)\end{array}$ & Reference & Comments \\
\hline $3^{1} S$ & 1.0 & & $\begin{array}{c}36 \\
49 \\
131.4\end{array}$ & $\begin{array}{l}108 \\
40 \\
32-33\end{array}$ & $\begin{array}{l}36.84 \\
31.9 \\
\ldots \\
22.3 \\
46.125\end{array}$ & $\begin{array}{l}u \\
v \\
w \\
x \\
z\end{array}$ & $\begin{array}{r}\text { b k } \\
\text { b g } \\
\text { b g } \\
\text { b h } \\
\text { Theoretical, m }\end{array}$ \\
\hline $4^{1} S$ & 0.59 & 15.1 & $\begin{array}{l}20 \\
16.5 \\
24 \\
25.4\end{array}$ & $\begin{array}{r}33 \\
108 \\
43 \\
33\end{array}$ & $\begin{array}{r}15.25 \\
16.88 \\
15.58 \\
15.41 \\
7.67 \\
17.81\end{array}$ & $\begin{array}{l}\mathrm{s} \\
\mathrm{u} \\
\mathrm{v} \\
\mathrm{w} \\
\mathrm{x} \\
\mathrm{z}\end{array}$ & $\begin{array}{r}\text { b g } \\
\text { b k } \\
\text { b g } \\
\text { bg } \\
\text { b h } \\
\text { Theoretical, } \mathrm{m}\end{array}$ \\
\hline$\overline{5}^{1} S$ & 0.471 & 5.8 & $\begin{array}{r}8.0 \\
7.0 \\
9.2 \\
12.3\end{array}$ & $\begin{array}{l}33 \\
108 \\
45 \\
33-34\end{array}$ & $\begin{array}{c}5.21 \\
7.16 \\
6.37 \\
\ldots . . \\
3.64 \\
8.78\end{array}$ & $\begin{array}{l}\mathrm{s} \\
\mathrm{u} \\
\mathrm{v} \\
\mathrm{w} \\
\mathrm{x} \\
\mathrm{z}\end{array}$ & $\begin{array}{r}\text { b g } \\
\text { b k } \\
\text { b g } \\
\text { b g } \\
\text { b h } \\
\text { Theoretical, } \mathrm{m}\end{array}$ \\
\hline $6^{\prime} S$ & 0.43 & 2.6 & $\begin{array}{l}4.0 \\
4.8 \\
6.0\end{array}$ & $\begin{array}{l}108 \\
45 \\
35-37\end{array}$ & $\begin{array}{l}4.09 \\
3.14 \\
\cdots \\
1.98 \\
4.93\end{array}$ & $\begin{array}{l}u \\
v \\
w \\
\mathbf{x} \\
z\end{array}$ & $\begin{array}{r}\text { b k } \\
\text { b g } \\
\text { b g } \\
\text { b h } \\
\text { Theoretical, } \mathrm{m}\end{array}$ \\
\hline $7^{1} S$ & $\sim 0.43$ & 1.5 & $\sim 3.5$ & $35-38$ & $\ddot{\cdots}$ & $\begin{array}{l}\mathrm{w} \\
\mathrm{z}\end{array}$ & $\begin{array}{r}\text { b g } \\
\text { Theoretical, } \mathrm{m}\end{array}$ \\
\hline $8^{1} S$ & $\sim 0.43$ & 1.1 & $\sim 2.5$ & $33-36$ & $\ddot{2.0}$ & $\begin{array}{l}\mathrm{w} \\
z\end{array}$ & $\begin{array}{r}\text { bg } \\
\text { Theoretical, } \mathrm{m}\end{array}$ \\
\hline
\end{tabular}




\begin{tabular}{|c|c|c|c|c|c|c|c|}
\hline \multirow[b]{2}{*}{$n^{1} D$} & \multicolumn{5}{|c|}{ (c) Helium $n^{1} D \rightarrow 2^{1} P$} & \multirow[b]{2}{*}{ Reference } & \multirow[b]{2}{*}{ Comments } \\
\hline & $\begin{array}{l}\text { Branching } \\
\text { ratio } \\
B(n \rightarrow 2)\end{array}$ & $\begin{array}{c}Q(n \rightarrow 2) \\
\left(10^{-20} \mathrm{~cm}^{2}\right)\end{array}$ & $\begin{array}{c}Q\left(n^{1} D\right) \\
=Q(n \rightarrow 2) / B(n \rightarrow 2) \\
\left(10^{-20} \mathrm{~cm}^{2}\right)\end{array}$ & $\begin{array}{l}\text { Electron } \\
\text { energya } \\
(\mathrm{eV})\end{array}$ & $\begin{array}{c}Q\left(n^{1} D\right) \\
\text { Translated } \\
\text { to } 100 \mathrm{eV} \\
\left(10^{-20} \mathrm{~cm}^{2}\right)\end{array}$ & & \\
\hline \multirow[t]{5}{*}{$3^{1} D$} & 1.0 & & 25 & 108 & 26.94 & $\mathrm{u}$ & $\mathrm{f} \mathrm{k}$ \\
\hline & & & 42 & 46 & 24.09 & $v$ & degin \\
\hline & & & 119.2 & $44-46$ & -. & $\mathrm{w}$ & d g i \\
\hline & & & & & 14.2 & $\mathrm{x}$ & $h \mathrm{jl}$ \\
\hline & & & & & 7.92 & $y$ & Theoretical, $\mathrm{m}$ \\
\hline \multirow[t]{6}{*}{$4^{1} D$} & 0.74 & 13 & 17.6 & $40-45$ & 11.88 & $\mathrm{~s}$ & deg in \\
\hline & & & 12 & 108 & 12.93 & $\mathrm{u}$ & $\mathrm{f} \mathrm{k}$ \\
\hline & & & 17.6 & 53 & 13.16 & $\mathrm{v}$ & $\mathrm{dg} \mathrm{i}$ \\
\hline & & 26.7 & 36.0 & $42-45$ & 27.22 & w & $\mathrm{dg}$ i \\
\hline & & & & & 6.75 & $\mathbf{x}$ & h j l \\
\hline & & & & & 4.13 & y & Theoretical, $\mathrm{m}$ \\
\hline \multirow[t]{6}{*}{$5^{1} D$} & 0.652 & 5.5 & 8.5 & $44-48$ & 5.70 & $\mathrm{~s}$ & deg in \\
\hline & & & 7.1 & 108 & 7.65 & u & $\mathrm{f} \mathrm{k}$ \\
\hline & & & 9.0 & 53 & 6.73 & $\mathrm{v}$ & $\mathrm{dg} i$ \\
\hline & & 7.9 & 12.1 & $40-45$ & $\cdots$ & $\mathbf{w}$ & $\mathrm{dg} i$ \\
\hline & & & & & 3.80 & $\mathrm{x}$ & $\mathrm{h} \mathrm{j} \mathrm{l}$ \\
\hline & & & & & 2.29 & $\mathbf{y}$ & Theoretical, m \\
\hline \multirow[t]{5}{*}{$6^{1} D$} & 0.617 & 3.0 & 4.9 & $42-46$ & 2.99 & $\mathrm{~s}$ & deg in \\
\hline & & & 3.0 & 108 & 3.24 & $\mathrm{u}$ & $\mathrm{fk}$ \\
\hline & & & 4.7 & 53 & 3.53 & $\mathrm{v}$ & $\mathrm{dg} i$ \\
\hline & & 5.3 & 8.6 & $40-45$ & $\ldots$ & $w$ & $\mathrm{dg} \mathrm{i}$ \\
\hline & & & & & 2.00 & $\mathrm{x}$ & $\mathrm{h} \mathrm{jl}$ \\
\hline \multirow[t]{2}{*}{$7^{1} D$} & $\sim 0.617$ & & 1.8 & 108 & 1.94 & u & $\mathrm{f} \mathrm{k}$ \\
\hline & & 3.0 & $\sim 5$ & $42-45$ & $\ldots$ & $\mathrm{w}$ & $\mathrm{dg} \mathrm{i}$ \\
\hline \multirow[t]{2}{*}{$8^{1} D$} & $\sim 0.617$ & & 1.4 & 108 & 1.51 & $\mathrm{u}$ & $\mathrm{f} \mathrm{k}$ \\
\hline & & 1.6 & $\sim 2.5$ & $41-46$ & $\cdots$ & w & $\mathrm{dg} \mathrm{i}$ \\
\hline \multicolumn{8}{|c|}{ (d) Helium $n^{3} S \rightarrow 2^{3} P$} \\
\hline$n^{3} S$ & $\begin{array}{l}\text { Branching } \\
\text { ratio } \\
B(n \rightarrow 2)\end{array}$ & $\begin{array}{c}Q(n \rightarrow 2) \\
\left(10^{-20} \mathrm{~cm}^{2}\right)\end{array}$ & $\begin{array}{c}Q\left(n^{3} S\right) \\
=Q(n \rightarrow 2) / B(n \rightarrow 2) \\
\left(10^{-20} \mathrm{~cm}^{2}\right)\end{array}$ & $\begin{array}{c}\text { Electron } \\
\text { energy } \\
(\mathrm{eV})\end{array}$ & $\begin{array}{c}Q\left(n^{3} S\right) \\
\text { Translated } \\
\text { to } 100 \mathrm{eV} \\
\left(10^{-20} \mathrm{~cm}^{2}\right)\end{array}$ & Reference & Comments \\
\hline \multirow[t]{5}{*}{$3^{3} S$} & 1.0 & & 15 & 108 & 14.68 & u & $\mathbf{k}$ \\
\hline & & & 107 & 35 & 11.06 & $\mathrm{v}$ & $\mathrm{g}$ \\
\hline & & & 130.1 & 26.5 & .. & $\mathrm{w}$ & $\mathrm{g}$ \\
\hline & & & & & 3.24 & $\mathrm{x}$ & $\mathrm{h}$ \\
\hline & & & & & 65.98 & $\mathbf{y}$ & Theoretical, $\mathrm{m}$ \\
\hline \multirow[t]{7}{*}{$4^{3} S$} & 0.62 & 15 & 24 & 26.5 & 2.51 & $\mathbf{s}$ & $\mathrm{g} \mathrm{n}$ \\
\hline & & 21.9 & 35 & 30 & $\ldots$ & $\mathrm{t}$ & $\mathrm{g} \mathrm{n}$ \\
\hline & & & 4.4 & 108 & 4.31 & $\mathrm{u}$ & $\mathrm{k}$ \\
\hline & & & 35 & 35 & 3.6 & $\mathbf{v}$ & $\mathrm{g}$ \\
\hline & & 22.0 & 35 & 27 & 4.95 & $\mathrm{w}$ & $\mathrm{g}$ \\
\hline & & & & & 1.51 & $\mathbf{x}$ & h \\
\hline & & & & & 2.46 & $\mathbf{y}$ & Theoretical, m \\
\hline \multirow[t]{7}{*}{$5^{3} S$} & 0.477 & 6.1 & 12.8 & 27 & 0.99 & s & $\mathrm{g} \mathrm{n}$ \\
\hline & & 8.2 & 17.2 & 30 & $\cdots$ & $\mathbf{t}$ & $\mathrm{gn}$ \\
\hline & & & 1.44 & 108 & 1.41 & u & k \\
\hline & & & 12.3 & 35 & 1.27 & $v$ & $\mathrm{~g}$ \\
\hline & & 8.0 & 17.0 & 28 & $\ldots$ & $\mathbf{w}$ & $\mathrm{g}$ \\
\hline & & & & & 0.451 & $\mathrm{z}$ & $\mathrm{h}$ \\
\hline & & & & & 1.23 & $\mathbf{y}$ & Theoretical, $\mathrm{m}$ \\
\hline \multirow[t]{2}{*}{$6^{8} S$} & 0.454 & 3.2 & 7.1 & 30 & $\cdots$ & $\mathrm{t}$ & $\mathrm{g} \mathrm{n}$ \\
\hline & & 3.7 & 8.2 & 29 & $\cdots$ & $\mathrm{w}$ & $\mathrm{g}$ \\
\hline \multirow[t]{3}{*}{$7^{3} S$} & $\sim 0.45$ & 1.4 & 3.1 & 30 & ... & $\mathbf{t}$ & $\mathrm{gn}$ \\
\hline & & & 1.06 & 108 & 1.04 & $\mathbf{u}$ & k \\
\hline & & 1.3 & 2.5 & 29 & $\ldots$ & $\mathbf{w}$ & $\mathrm{g}$ \\
\hline
\end{tabular}


TABLE LXIV (Continued)

(e) Helium $n^{3} P \rightarrow 2^{3} S$

\begin{tabular}{|c|c|c|c|c|c|c|c|}
\hline$n^{3} P$ & $\begin{array}{l}\text { Branching } \\
\text { ratio } \\
B(n \rightarrow 2)\end{array}$ & $\begin{array}{c}Q(n \rightarrow 2) \\
\left(10^{-20} \mathrm{~cm}^{2}\right)\end{array}$ & $\begin{array}{c}\text { (e) Heli } \\
Q\left(n^{3} P\right) \\
=Q(n \rightarrow 2) / B(n \rightarrow 2) \\
\left(10^{-20} \mathrm{~cm}^{2}\right)\end{array}$ & $\begin{array}{c}\text { Electron } \\
\text { energy“ } \\
(\mathrm{eV})\end{array}$ & $\begin{array}{c}Q\left(n^{3} P\right) \\
\text { Translated } \\
\text { to } 100 \mathrm{eV} \\
\left(10^{-20} \mathrm{~cm}^{2}\right)\end{array}$ & Reference & Comments \\
\hline $2^{3} P$ & 1.0 & 2510 & & 27 & $\ldots$ & $\mathrm{w}$ & $\mathrm{d} g \mathrm{i}$ \\
\hline \multirow[t]{7}{*}{${ }^{3} P$} & 0.898 & 75 & 84 & 28 & 11.21 & $\mathbf{s}$ & deg in \\
\hline & & 95 & 106 & 32 & $\ldots$ & $\mathbf{t}$ & degin \\
\hline & & & 11 & 108 & 13.41 & $\mathrm{u}$ & $\mathrm{f} \mathrm{k}$ \\
\hline & & & 97 & 37 & 17.13 & $\mathbf{v}$ & $\mathrm{dg} \mathrm{i}$ \\
\hline & & 121 & 135 & 28 & 33.81 & w & $\mathrm{dg} i$ \\
\hline & & & & & 5.01 & $\mathrm{x}$ & $h \mathrm{jl}$ \\
\hline & & & & & 4.84 & $\mathrm{y}$ & Theoretical, m \\
\hline \multirow[t]{5}{*}{$4^{3} P$} & 0.779 & 10.3 & 13 & 32 & $\ldots$ & $\mathrm{t}$ & deg in \\
\hline & & & 4 & 108 & 4.88 & $\mathbf{u}$ & $\mathrm{f} \mathrm{k}$ \\
\hline & & 8 & 10 & 29 & $\ldots$ & $\mathrm{w}$ & d g i \\
\hline & & & & & 1.74 & $x$ & $\quad h \mathbf{j} l$ \\
\hline & & & & & 2.02 & $y$ & Theoretical, $\mathrm{m}$ \\
\hline \multirow[t]{4}{*}{$5^{3} P$} & 0.686 & & 2 & 108 & 2.44 & u & $\mathrm{f} \mathrm{k}$ \\
\hline & & $\sim 0.5$ & 0.75 & 30 & $\ldots$ & $\mathbf{w}$ & $d g i$ \\
\hline & & & & & 0.987 & $\mathrm{x}$ & $h \mathbf{j l}$ \\
\hline & & & & & 1.06 & $y$ & Theoretical, m \\
\hline
\end{tabular}

(f) Helium $n^{3} D \rightarrow 2^{3} P$

$Q\left(n^{3} D\right)$

\begin{tabular}{|c|c|c|c|c|c|c|c|}
\hline$n^{3} D$ & $\begin{array}{c}\text { Branching } \\
\text { ratio } \\
B(n \rightarrow 2) \\
\end{array}$ & $\begin{array}{c}Q(n \rightarrow 2) \\
\left(10^{-20} \mathrm{~cm}^{2}\right)\end{array}$ & $\begin{aligned} & Q\left(n^{3} D\right) \\
&= Q(n \rightarrow 2) / B(n \rightarrow 2) \\
&\left(10^{-20} \mathrm{~cm}^{2}\right) \\
&\end{aligned}$ & $\begin{array}{l}\text { Electron } \\
\text { energya } \\
(\mathrm{eV})\end{array}$ & $\begin{array}{l}\text { Translated } \\
\text { to } 100 \mathrm{eV} \\
\left(10^{-20} \mathrm{~cm}^{2}\right) \\
\end{array}$ & Reference & Comments \\
\hline \multirow[t]{6}{*}{$3^{3} D$} & 1.0 & & 36 & 26 & 9.04 & $\mathbf{s}$ & degin \\
\hline & & & 25 & 108 & 28.35 & $\mathbf{u}$ & $\mathrm{f} \mathrm{k}$ \\
\hline & & & 31 & 35 & 4.55 & $\mathbf{v}$ & $\mathrm{d} g \mathrm{i}$ \\
\hline & & & 35 & 27 & $\cdots$ & $\mathbf{w}$ & $\mathrm{dg}$ i \\
\hline & & & & & 1.61 & $\mathrm{x}$ & h j l \\
\hline & & & & & 0.11 & $y$ & Theoretical, m \\
\hline \multirow[t]{6}{*}{$4^{3} D$} & 0.789 & 9.8 & 12.4 & 27 & 2.58 & s & de $g$ i n \\
\hline & & & 4.6 & 108 & 5.22 & $\mathrm{u}$ & $\mathrm{f} \mathrm{k}$ \\
\hline & & & 12.0 & 35 & 1.74 & $\mathbf{v}$ & $\mathrm{dg} \mathrm{i}$ \\
\hline & & 11.6 & 14.7 & 27.5 & 5.44 & $\mathbf{w}$ & $\mathrm{dg} \mathrm{i}$ \\
\hline & & & & & 0.471 & $\mathrm{x}$ & h j l \\
\hline & & & & & 0.063 & $\mathrm{y}$ & Theoretical, m \\
\hline \multirow[t]{5}{*}{$5^{3} D$} & 0.713 & 6.1 & 8.6 & $30-32$ & 3.78 & s & $\mathrm{deg}$ i n \\
\hline & & & 3.0 & 108 & 3.41 & $\mathrm{u}$ & $\mathrm{f} \mathrm{k}$ \\
\hline & & & 6.2 & 38 & 0.92 & $\mathbf{v}$ & $d g i$ \\
\hline & & 6.4 & 9.0 & 29.0 & $\ldots$ & $\mathrm{w}$ & $\mathrm{d} \mathrm{g} \mathrm{i}$ \\
\hline & & & & & 0.037 & $\mathrm{y}$ & Theoretical, $\mathrm{m}$ \\
\hline \multirow[t]{3}{*}{$6^{3} D$} & 0.657 & & 1.5 & 108 & 1.70 & u & $\mathrm{f} \mathrm{k}$ \\
\hline & & & 3.9 & 35 & 0.56 & $\mathbf{v}$ & $\mathrm{dg}$ i \\
\hline & & 3.7 & 5.6 & 29.0 & $\ldots$ & $\mathbf{w}$ & d g i \\
\hline \multirow[t]{2}{*}{$7^{3} \mathrm{D}$} & $\sim 0.65$ & & 0.7 & 108 & 0.80 & $\mathrm{u}$ & $\mathrm{f} \mathrm{k}$ \\
\hline & & 1.7 & $\sim 2.6$ & $29-30$ & $\ldots$ & $\mathbf{w}$ & $\mathrm{dg} \mathrm{i}$ \\
\hline $8^{3} D$ & $\sim 0.65$ & 0.8 & $\sim 0.01$ & $29-30$ & $\ldots$ & $\mathbf{w}$ & d g i \\
\hline
\end{tabular}

s Possible error due to imprisonment of resonance radiation.

b Substantial error due to imprisonment of resonance radiation.

- Complete imprisonment of resonance radiation.

d Possible large instrumental polarization error.

- Some pressure depolarization likely.

f Essentially complete pressure depolarization.

Uncorrected for cascading.

Correction for cascading applied by author.

Uncorrected for polarization.

j Correction for polarization applied by author.

Extrapolated to zero pressure from data taken at several pressures ranging from about 0.01 to 0.1 torr.

1 Correction for instrumental polarization applied by author.

m Theoretical values do not include cascading contributions.

n Possible error due to excitation transfer.

- Branching ratios calculated from Wiese, Smith, and Glennon (1966).

D Values presented in boldface are numbers which appear in the original

a These are the energies at which the maximum excitation was observed except for the data (Ref. 3) of Gabriel and Heddle for which observations were made at $108 \mathrm{eV}$.

Data of Gabriel and Heddle (1960) was translated from 108 to $100 \mathrm{eV}$ using the excitation function from the appropriate series as measured by Heddle and Lucas (1963). Since the translation amounted to $8 \mathrm{eV}$ a negligible error is introduced. Other translations were based on curves presented in the same papers as the original absolute values.

- Yakhontova $(1959,1960)$

Stewart and Gabathuler (1959).

4 Gabriel and Heddle (1960)

St. John, Miller, and Lin (1964); Miller (1964).

- Zapesochnyi and Feltsan (1965).

I Moustafa Moussa (1967)

Ochkur and Bratsev (1965b)

Fox (1965). 


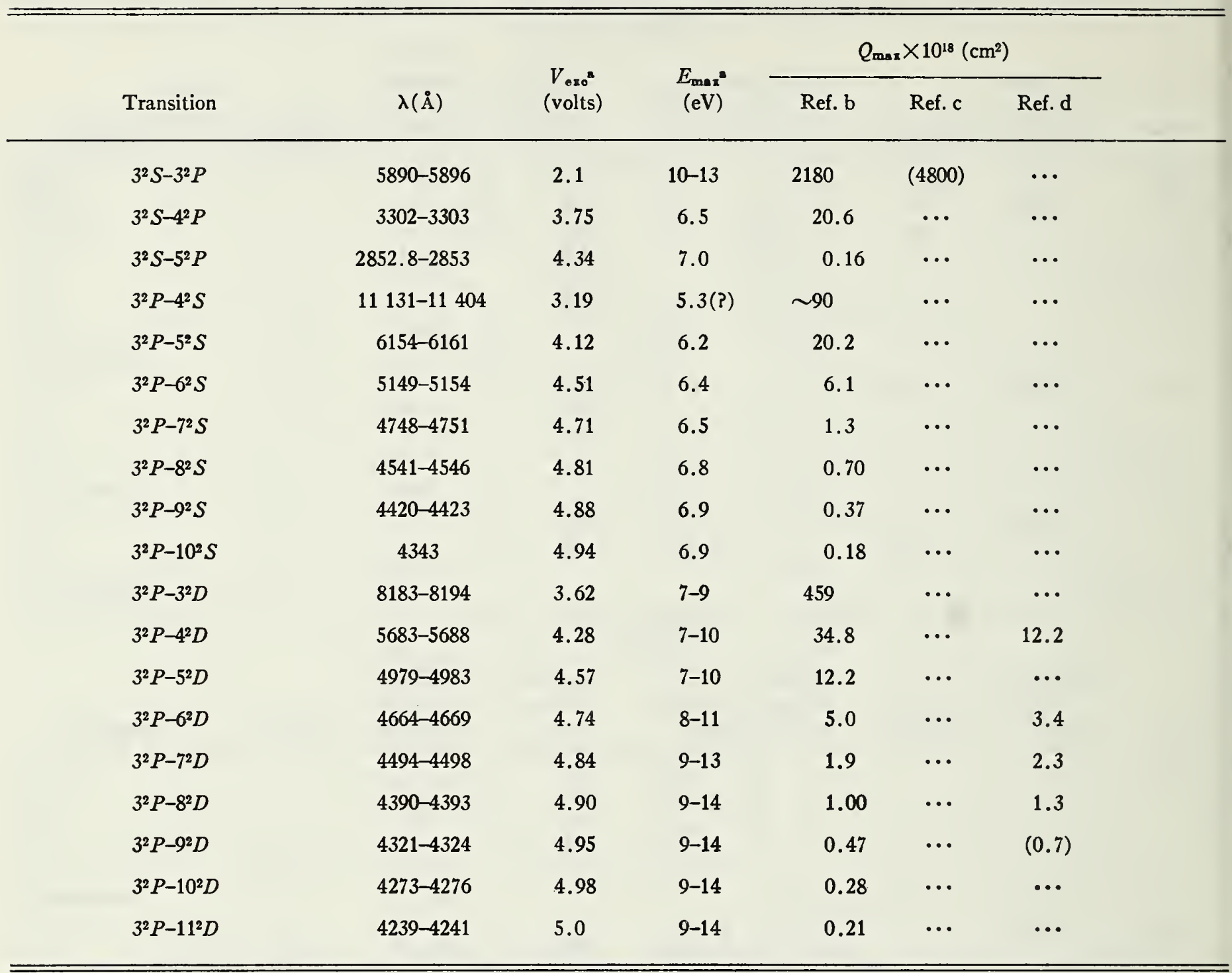

- Nole: $V_{\text {exo }}$ (volts) excitation potential; $E_{\max }(\mathrm{eV})$-location of the Christoph (1935). maximum of the excitation function.

bapesochnyi and Shimon (1965).

d Volkova (1961).

(2) 


\section{NBS TECHNICAL PUBLICATIONS}

\section{PERIODICALS}

JOURNAL OF RESEARCH reports National Bureau of Standards research and development in physics, mathematics, chemistry, and engineering. Comprehensive scientific papers give complete details of the work, including laboratory data, experimental procedures, and theoretical and mathematical analyses. Illustrated with photographs, drawings, and charts.

\section{Published in three sections, available separately:}

\section{- Physics and Chemistry}

Papers of interest primarily to scientists working in these fields. This section covers a broad range of physical and chemical research, with major emphasis on standards of physical measurement, fundamental constants, and properties of matter. Issued six times a year. Annual subscription: Domestic, $\$ 5.00$; foreign, $\$ 6.00^{*}$.

\section{- Mathematical Sciences}

Studies and compilations designed mainly for the mathematician and theoretical physicist. Topics in mathematical statistics, theory of experiment design, numerical analysis, theoretical physics and chemistry, logical design and programming of computers and computer systems. Short numerical tables. Issued quarterly. Annual subscription: Domestic, $\$ 2.25$; foreign, $\$ 2.75 *$.

\section{- Engineering and Instrumentation}

Reporting results of interest chiefly to the engineer and the applied scientist. This section includes many of the new developments in instrumentation resulting from the Bureau's work in physical measurement, data processing, and development of test methods. It will also cover some of the work in acoustics, applied mechanics, building research, and cryogenic engineering. Issued quarterly. Annual subscription: Domestic, $\$ 2.75$; foreign, $\$ 3.50 *$.

\section{TECHNICAL NEWS BULLETIN}

The best single source of information concerning the Bureau's research, developmental, cooperative and publication activities, this monthly publication is designed for the industry-oriented individual whose daily work involves intimate contact with science and technology-for engineers, chemists, physicists, research managers, product-development managers, and company executives. Annual subscription: Domestic, $\$ 1.50$; foreign, $\$ 2.25^{*}$.

-Difference in price is due to extra cost of foreign mailing.

\section{NONPERIODICALS}

Applied Mathematics Series. Mathematical tables, manuals, and studies.

Building Science Series. Research results, test methods, and performance criteria of building materials, components, systems, and structures.

Handbooks. Recommended codes of engineering and industrial practice (including safety codes) developed in cooperation with interested industries, professional organizations, and regulatory bodies.

Special Publications. Proceedings of NBS conferences, bibliographies, annual reports, wall charts, pamphlets, etc.

Monographs. Major contributions to the technical literature on various subjects related to the Bureau's scientific and technical activities.

National Standard Reference Data Series. NSRDS provides quantitative data on the physical and chemical properties of materials, compiled from the world's literature and critically evaluated.

Product Standards. Provide requirements for sizes, types, quality and methods for testing various industrial products. These standards are developed cooperatively with interested Government and industry groups and provide the basis for common understanding of product characteristics for both buyers and sellers. Their use is voluntary.

Technical Notes. This series consists of communications and reports (covering both other agency and NBS-sponsored work) of limited or transitory interest.

\section{CLEARINGHOUSE}

The Clearinghouse for Federal Scientific and Technical Information, operated by NBS, supplies unclassified information related to Governmentgenerated science and technology in defense, space, atomic energy, and other national programs. For further information on Clearinghouse services, write:

Clearinghouse

U.S. Department of Commerce

Springfield, Virginia 22151

Order NBS publications from:

Superintendent of Documents

Government Printing Office

Washington, D.C. 20402 


\section{National Standard Reference Data Series National Bureau of Standards}

National Standard Reference Data System, Plan of Operation, NSRDS-NBS 115 cents*

Thermal Properties of Aqueous Uni-univalent Electrolytes, NSRDS-NBS 2-45 cents*

Selected Tables of Atomic Spectra, Atomic Energy Levels and Multiplet TablesSi II, Si III, Si IV, NSRDS-NBS 3, Section 1-35 cents*

Selected Tables of Atomic Spectra, Atomic Energy Levels and Multiplet TablesSi I, NRSDS-NBS 3, Section 2-20 cents*

Atomic Transition Probabilities, Volume I, Hydrogen Through Neon, NSRDS-NBS $4-\$ 2.50^{*}$

The Band Spectrum of Carbon Monoxide, NSRDS-NBS $5-70$ cents*

Tables of Molecular Vibrational Frequencies, Part 1, NSRDS-NBS 6-40 cents*

High Temperature Properties and Decomposition of Inorganic Salts, Part 1, Sulfates, NSRDS-NBS $7-35$ cents*

Thermal Conductivity of Selected Materials, NSRDS-NBS $8-\$ 1.00 *$

Tables of Biomolecular Gas Reactions, NSRDS-NBS 9-\$2.00*

Selected Values of Electric Dipole Moments for Molecules in the Gas Phase, NSRDS-NBS $10-40$ cents*

Tables of Molecular Vibrational Frequencies, Part 2, NSRDS-NBS $11-30$ cents*

Tables for the Rigid Asymmetric Rotor: Transformation Coefficient for Symmetric to Asymmetric Bases and Expectation Values of $\mathrm{P}_{z}^{2}, \mathrm{P}_{z}^{4}$, and $\mathrm{P}_{z}^{6}$, NSRDS-NBS $12-60$ cents*

Hydrogenation of Ethylene on Metallic Catalysts, NSRDS-NBS $13-\$ 1.00$

X-Ray Wavelengths and X-Ray Atomic Energy Levels, NSRDS-NBS $14-40$ cents*

Molten Salts: Electrical Conductance, Density and Viscosity Data, NSRDS-NBS 15-In press

Thermal Conductivity of Selected Materials, Part 2, NSRDS-NBS $16-\$ 2.00$ *

Tables of Molecular Vibrational Frequencies, Part 3, NSRDS-NBS $17-30$ cents*

Critical Analysis of the Heat-Capacity Data of the Literature and Evaluation of Thermodynamic Properties of Copper, Silver, and Gold from 0 to $300^{\circ} \mathrm{K}$, NSRDS-NBS $18-40$ cents*

Thermodynamic Properties of Ammonia as an Ideal Gas, NSRDS-NBS 19-In press

Gas Phase Reaction Kinetics of Neutral Oxygen Species, NSRDS-NBS 20-In press

Atomic Transition Probabilities (A Critical Data Compilation). Vol. II, NSRDSNBS 22-In press

Partial Grotrian Diagrams of Astrophysical Interest, NSRDS-NBS 23-In press

Theoretical Mean Activity Coefficients of Strong Electrolytes in Aqueous Solutions from $0{ }^{\circ} \mathrm{C}$ to $100{ }^{\circ} \mathrm{C}$, NSRDS-NBS 24 -In press

*Send orders with remittance to: Superintendent of Documents, U.S. Government Printing Office, Washington, D.C. 20402. Remittances from foreign countries should include an additional one-fourth of the purchase price for postage. 


\section{Announcement of New Publications in National Standard Reference Data Series}

Superintendent of Documents, Government Printing Office,

Washington, D.C. 20402

\section{Dear Sir:}

Please add my name to the announcement list of new publications to be issued in the series: National Standard Reference Data Series-National Bureau of Standards.

Name

Company.

Address.

City. State Zip Code

(Notification key N-337) 


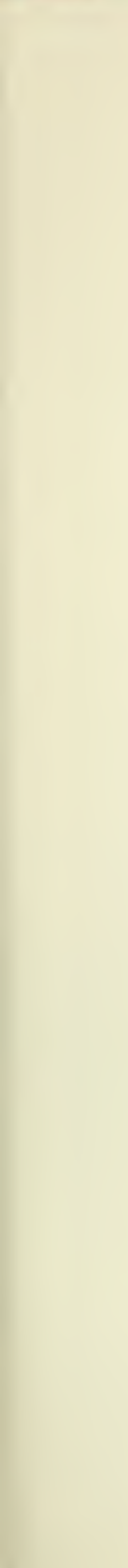


